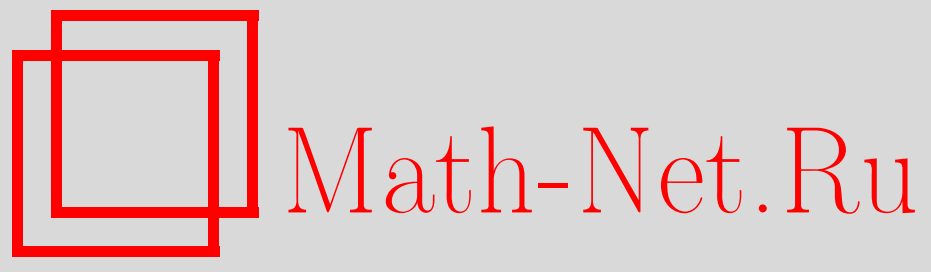

И. А. Чельцов, К. А. Шрамов, Лог-канонические пороги неособых трехмерных многообразий Фано, УМН, 2008, том 63, выпуск 5, 73-180

DOI: https://doi.org/10.4213/rm9235

Использование Общероссийского математического портала Math-Net.Ru подразумевает, что вы прочитали и согласны с пользовательским соглашением http://www . mathnet.ru/rus/agreement

Параметры загрузки:

IP : 54.92 .164 .108

26 апреля 2023 г., 06:15:26

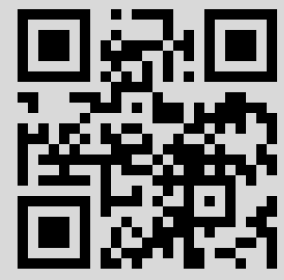




\title{
Лог-канонические пороги неособых трехмерных многообразий Фано
}

\begin{abstract}
И. А. Чельцов, К. А. Шрамов
Комплексный показатель особенности является локальным инвариантом голоморфной функции, который может быть определен в терминах интегрируемости дробных степеней данной функции. Лог-канонические пороги эффективных $\mathbb{Q}$-дивизоров на нормальных алгебраических многообразиях суть алгебраические аналоги комплексных показателей особенности. В случае многообразий Фано у этих инвариантов есть глобальные аналоги. В первом случае это так называемый $\alpha$-инвариант Тиана, а во втором - глобальный лог-канонический порог многообразия Фано, равный инфимуму лог-канонических порогов всех эффективных $\mathbb{Q}$-дивизоров, численно эквивалентных антиканоническому дивизору. В приложении к настоящей работе показано, что глобальный лог-канонический порог неособого многообразия Фано совпадает с его $\alpha$-инвариантом Тиана. Основная цель работы - найти глобальные лог-канонические пороги неособых трехмерных многообразий Фано (всего существует 105 деформационных семейств таких многообразий). В работе найдено значение глобального лог-канонического порога всех многообразий из 64 деформационных семейств и общих многообразий из еще 20 деформационных семейств. Для многообразий из 14 семейств получены оценки на возможные значения глобальных лог-канонических порогов. Приложение А написано Ж.-П. Демайи.

Библиография: 73 названия.
\end{abstract}

\section{СоДЕРЖАНИЕ}

1. Введение.............................................. 74

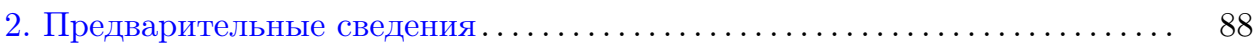

3. Кубические поверхности . ............................. 98

4. Поверхности дель Пеццо ................................. 107

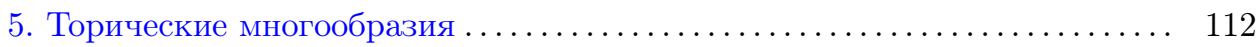

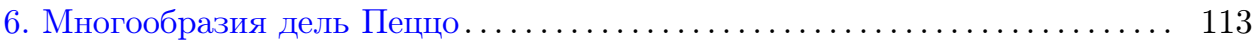

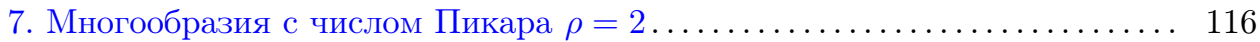

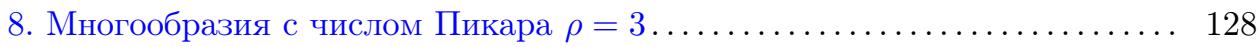

9. Многообразия с числом Пикара $\rho \geqslant 4 \ldots \ldots \ldots \ldots \ldots \ldots \ldots \ldots \ldots \ldots \ldots$

Работа выполнена при поддержке грантов NSF DMS-0701465 и EPSRC EP/E048412/1, а также грантов РФФИ № 08-01-00395-а и НШ-1987.2008.1.

(С) И. А. ЧЕльцов, К. А. Шрамов, 2008 
10. Оценки сверху.......................................... 160 Приложение А.

Ж.-П. Демайи. Инвариант Тиана и лог-канонические пороги ... 165

Приложение В. Большая таблица.............................. 172

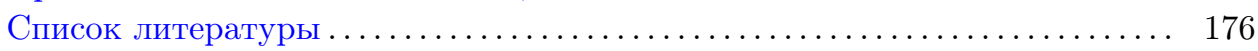

\section{1. Введение}

Кратность многочлена $\varphi \in \mathbb{C}\left[z_{1}, z_{2}, \ldots, z_{n}\right]$ в начале координат $O \in \mathbb{C}^{n}$ есть число

$$
\min \left\{m \in \mathbb{Z}_{\geqslant 0} \mid \frac{\partial^{m} \varphi\left(z_{1}, z_{2}, \ldots, z_{n}\right)}{\partial^{m_{1}} z_{1} \partial^{m_{2}} z_{2} \cdots \partial^{m_{n}} z_{n}}(O) \neq 0\right\} \in \mathbb{Z}_{\geqslant 0} \cup\{+\infty\} .
$$

Более тонким инвариантом является число $c_{0}(\varphi) \in \mathbb{Q} \cup\{+\infty\}$, определяемое формулой

$c_{0}(\varphi)=\sup \left\{\varepsilon \in \mathbb{Q} \mid\right.$ функция $|\varphi|^{-2 \varepsilon}$ интегрируема в окрестности точки $\left.O \in \mathbb{C}^{n}\right\}$,

которое называют комплексным показателем особенности многочлена $\varphi$ в точке $O$.

ПримеР 1.1. Пусть $m_{1}, m_{2}, \ldots, m_{n}$ - произвольные положительные целые числа. Тогда

$$
\min \left(1, \sum_{i=1}^{n} \frac{1}{m_{i}}\right)=c_{0}\left(\sum_{i=1}^{n} z_{i}^{m_{i}}\right) \geqslant c_{0}\left(\prod_{i=1}^{n} z_{i}^{m_{i}}\right)=\min \left(\frac{1}{m_{1}}, \frac{1}{m_{2}}, \ldots, \frac{1}{m_{n}}\right) .
$$

Пусть $X$ - алгебраическое многообразие ${ }^{1}$, которое имеет не более чем лог-канонические особенности (см. [1]), пусть $Z \subseteq X$ - некоторое непустое замкнутое подмногообразие, и пусть $D$ - эффективный $\mathbb{Q}$-Картье $\mathbb{Q}$-дивизор на многообразии $X$. Тогда число

$\operatorname{lct}_{Z}(X, D)=\sup \{\lambda \in \mathbb{Q} \mid$ лог-пара $(X, \lambda D)$ лог-канонична вдоль $Z\} \in \mathbb{Q} \cup\{+\infty\}$

называется лог-каноническим порогом дивизора $D$ вдоль $Z$. В работе [1] показано, что

$$
\operatorname{lct}_{O}\left(\mathbb{C}^{n},(\varphi=0)\right)=c_{0}(\varphi),
$$

т. е. $\operatorname{lct}_{Z}(X, D)$ - обобщение числа $c_{0}(\varphi)$. Положим $\operatorname{lct}(X, D)=\operatorname{lct}_{X}(X, D)$. Тогда

$$
\begin{aligned}
\operatorname{lct}(X, D) & =\inf \left\{\operatorname{lct}_{P}(X, D) \mid P \in X\right\} \\
& =\sup \{\lambda \in \mathbb{Q} \mid \text { лог-пара }(X, \lambda D) \text { лог-канонична }\} .
\end{aligned}
$$

Пусть $X$ - многообразие Фано (см. [2]) с лог-терминальными особенностями.

\footnotetext{
${ }^{1}$ Все многообразия считаются проективными, нормальными и определенными над полем $\mathbb{C}$.
} 
ОПРЕДЕЛЕНИЕ 1.2. Глобальный лог-канонический порог многообразия Фано $X$ есть число

$$
\begin{gathered}
\operatorname{lct}(X)=\inf \{\operatorname{lct}(X, D) \mid D-\text { эффективный } \mathbb{Q} \text {-дивизор на } X \\
\text { такой, что } \left.D \sim_{\mathbb{Q}}-K_{X}\right\} \geqslant 0 .
\end{gathered}
$$

Число $\operatorname{lct}(X)$ является алгебраическим аналогом так называемого $\alpha$-инварианта многообразия $X$, который был определен в работе [3]. Легко видеть, что выполнено равенство

$$
\begin{aligned}
\operatorname{lct}(X)=\sup \{\varepsilon \in \mathbb{Q} \mid & \text { лог-пара }\left(X, n^{-1} \varepsilon D\right) \text { лог-канонична для } \\
& \text { каждого дивизора } \left.D \in\left|-n K_{X}\right| \text { при всех } n \in \mathbb{Z}_{>0}\right\} .
\end{aligned}
$$

Группа $\operatorname{Pic}(X)$ свободна от кручения, так как $X$ рационально связно (см. [4]). Имеем

$$
\begin{array}{r}
\operatorname{lct}(X)=\sup \{\lambda \in \mathbb{Q} \mid \\
\text { длог-пара }(X, \lambda D) \text { имеет лог-канонические особенности } \\
\text { длждого эффективного } \left.\mathbb{Q} \text {-дивизора } D \equiv-K_{X}\right\} .
\end{array}
$$

ПримеР 1.3. Пусть $X$ - неособая гиперповерхность в $\mathbb{P}^{n}$ степени $m<n$. Тогда выполнено равенство $\operatorname{lct}(X)=1 /(n+1-m)$ (см. [5]). В частности, имеем $\operatorname{lct}\left(\mathbb{P}^{n}\right)=1 /(n+1)$.

ПРимеР 1.4. Пусть $X$ - рациональное однородное пространство такое, что $\operatorname{Pic}(X)=\mathbb{Z}[D]$, где $D$ - обильный дивизор. Тогда $\operatorname{lct}(X)=1 / r$ (см. [6]), где $-K_{X} \sim r D$ и $r \in \mathbb{Z}_{>0}$.

В общем случае число $\operatorname{lct}(X)$ меняется при малых деформациях многообразия $X$.

Пример 1.5. Пусть $X$ - неособая гиперповерхность в $\mathbb{P}(1,1,1,1,3)$ степени 6. Тогда

$$
\operatorname{lct}(X) \in\left\{\frac{5}{6}, \frac{43}{50}, \frac{13}{15}, \frac{33}{38}, \frac{7}{8}, \frac{8}{9}, \frac{9}{10}, \frac{11}{12}, \frac{13}{14}, \frac{15}{16}, \frac{17}{18}, \frac{19}{20}, \frac{21}{22}, \frac{29}{30}, 1\right\}
$$

согласно работам [7] и [8]. Все эти значения числа $\operatorname{lct}(X)$ реализуются.

Пример 1.6. Пусть $X$ - неособая гиперповерхность в $\mathbb{P}^{n}$ степени $n \geqslant 2$. Тогда, согласно работе [5],

$$
1 \geqslant \operatorname{lct}(X) \geqslant 1-1 / n
$$

В работах [7] и [8] показано, что для общей гиперповерхности $X$

$$
\operatorname{lct}(X) \geqslant \begin{cases}1, & \text { если } n \geqslant 6, \\ 22 / 25, & \text { если } n=5, \\ 16 / 21, & \text { если } n=4, \\ 3 / 4, & \text { если } n=3,\end{cases}
$$

при этом если $X$ содержит конус размерности $n-2$, то выполнено равенство $\operatorname{lct}(X)=1-1 / n$. 
Пример 1.7. Пусть $X$ - квазигладкая гиперповерхность в $\mathbb{P}\left(1, a_{1}, \ldots, a_{4}\right)$ степени $\sum_{i=1}^{4} a_{i}$, имеющая терминальные особенности, где $a_{1} \leqslant a_{2} \leqslant a_{3} \leqslant a_{4}$. Тогда $-\left.K_{X} \sim \mathscr{O}_{\mathbb{P}\left(1, a_{1}, \ldots, a_{4}\right)}(1)\right|_{X}$, а для четверки $\left(a_{1}, a_{2}, a_{3}, a_{4}\right)$ имеется 95 возможностей (см. [9], [10]). Если $X$ общая, то

$$
1 \geqslant \operatorname{lct}(X) \geqslant \begin{cases}16 / 21, & \text { если } a_{1}=a_{2}=a_{3}=a_{4}=1, \\ 7 / 9, & \text { если }\left(a_{1}, a_{2}, a_{3}, a_{4}\right)=(1,1,1,2), \\ 4 / 5, & \text { если }\left(a_{1}, a_{2}, a_{3}, a_{4}\right)=(1,1,2,2), \\ 6 / 7, & \text { если }\left(a_{1}, a_{2}, a_{3}, a_{4}\right)=(1,1,2,3), \\ 1 & \text { во всех оставшихся случаях }\end{cases}
$$

(см. [11], [8], [12]). Лог-канонический порог гиперповерхности

$$
w^{2}=t^{3}+z^{9}+y^{18}+x^{18} \subset \mathbb{P}(1,1,2,6,9) \cong \operatorname{Proj}(\mathbb{C}[x, y, z, t, w])
$$

равен $17 / 18$ (см. [11]), где $\operatorname{wt}(x)=\operatorname{wt}(y)=1, \operatorname{wt}(z)=2, \operatorname{wt}(t)=6, \operatorname{wt}(w)=9$.

ПримеР 1.8. Из леммы 5.1 следует, что $\operatorname{lct}\left(\mathbb{P}\left(a_{0}, a_{1}, \ldots, a_{n}\right)\right)=a_{0} / \sum_{i=0}^{n} a_{i}$ при условии, что пространство $\mathbb{P}\left(a_{0}, a_{1}, \ldots, a_{n}\right)$ хорошо сформировано (см. [9]) и $a_{0} \leqslant a_{1} \leqslant \cdots \leqslant a_{n}$.

ПримеР 1.9. Пусть $X$ - неособая гиперповерхность в $\mathbb{P}\left(1^{n+1}, d\right)$ степени $2 d$. Если $2 \leqslant d \leqslant n-1$, то выполнено равенство $\operatorname{lct}(X)=1 /(n+1-d)$ (см. [13; предложение 20]).

ПримеР 1.10. Пусть $X$ - неособая поверхность дель Пеццо. В работе [14] показано, что

$$
\operatorname{lct}(X)=\left\{\begin{array}{cc}
1, & \text { если } K_{X}^{2}=1 \text { и }\left|-K_{X}\right| \text { не содержит каспидальную кривую, } \\
5 / 6, & \text { если } K_{X}^{2}=1 \text { и }\left|-K_{X}\right| \text { содержит каспидальную кривую, } \\
5 / 6, & \text { если } K_{X}^{2}=2 \text { и }\left|-K_{X}\right| \text { не содержит такнодальную кривую, } \\
3 / 4, & \text { если } K_{X}^{2}=2 \text { и }\left|-K_{X}\right| \text { содержит такнодальную кривую, } \\
3 / 4, & \text { если } X \text { является кубикой в } \mathbb{P}^{3}, \text { не содержащей } \\
2 / 3, & \text { если } X \text { является Кубикой в } \mathbb{P}^{3} \text { с точкой Эккарда, } \\
& \text { либо } K_{X}^{2}=4, \\
1 / 2, & \text { если } X \cong \mathbb{P}^{1} \times \mathbb{P}^{1} \text { или } K_{X}^{2} \in\{5,6\}, \\
1 / 3 & \text { в оставшихся случаях. }
\end{array}\right.
$$

Было бы интересно найти глобальные лог-канонические пороги поверхностей дель Пеццо ранга Пикара 1, которые имеют не более чем канонические особенности (см. [15]).

ПримеР 1.11. Пусть $X$ - особая кубическая поверхность в $\mathbb{P}^{3}$, имеющая канонические особенности. Конфигурации особенностей поверхности $X$ описаны 
в [16]. В [17] показано, что

$$
\operatorname{lct}(X)=\left\{\begin{aligned}
2 / 3, & \text { если } \operatorname{Sing}(X)=\left\{\mathbb{A}_{1}\right\}, \\
1 / 3, & \text { если } \operatorname{Sing}(X) \supseteq\left\{\mathbb{A}_{4}\right\} \text { или } \operatorname{Sing}(X)=\left\{\mathbb{D}_{4}\right\} \\
1 / 4, & \text { или } \operatorname{Sing}(X) \supseteq\left\{\mathbb{A}_{2}, \mathbb{A}_{2}\right\} \\
1 / 6, & \text { если } \operatorname{Sing}(X) \supseteq\left\{\mathbb{A}_{5}\right\} \text { или } \operatorname{Sing}(X)=\left\{\mathbb{E}_{6}\right\}, \\
1 / 2 & \text { в оставшихся случаях. }
\end{aligned}\right.
$$

Рационально ли число $\operatorname{lct}(X)$, пока неизвестно ${ }^{2}$ (cp. [18; вопрос 1]).

ГиПотезА 1.12. Существует эббективный $\mathbb{Q}$-дивизор $D \sim_{\mathbb{Q}}-K_{X}$ на многообразии $X$ такой, что $\operatorname{lct}(X)=\operatorname{lct}(X, D) \in \mathbb{Q}$.

Пусть $G \subset \operatorname{Aut}(X)$ - произвольная подгруппа.

ОПРЕДЕЛЕНИЕ 1.13. Глобалъным $G$-инвариантным лог-каноническим порогом многообразия Фано $X$ будем называть число или $+\infty$, определенное посредством равенства

$$
\begin{aligned}
\operatorname{lct}(X, G)=\sup \{\lambda \in \mathbb{Q} \mid & \text { лог-пара }\left(X, n^{-1} \varepsilon \mathscr{D}\right) \text { лог-канонична для каждой } \\
& G \text {-инвариантной линейной подсистемы } \mathscr{D} \subset\left|-n K_{X}\right| \\
& \text { при всех } \left.n \in \mathbb{Z}_{>0}\right\} .
\end{aligned}
$$

ЗАмЕчАниЕ 1.14. В определениях 1.2 и 1.13 достаточно предполагать, что $\left|-n K_{X}\right| \neq \varnothing$ для некоторого $n \gg 0$. Последнее выполнено, например, для торических многообразий и для слабых многообразий Фано. Однако известные применения чисел $\operatorname{lct}(X)$ и $\operatorname{lct}(X, G)$ предполагают обильность дивизора $-K_{X}$ и компактность группы $G$.

В приложении А показано, что если $X$ неособо, а группа $G$ компактна, то выполнено равенство $\operatorname{lct}(X, G)=\alpha_{G}(X)$, где $\alpha_{G}(X)-\alpha$-инвариант Тиана, введенный в [3]. Отметим, что

$$
\begin{aligned}
\operatorname{lct}(X, G)=\sup \{\lambda \in \mathbb{Q} \mid & \text { лог-пара }(X, \lambda D) \text { лог-канонична для каждого эффек- } \\
& \text { тивного } \left.G \text {-инвариантного } \mathbb{Q} \text {-дивизора } D \sim_{\mathbb{Q}}-K_{X}\right\}
\end{aligned}
$$

в случае, когда $|G|<+\infty$. Очевидно, что $0 \leqslant \operatorname{lct}(X) \leqslant \operatorname{lct}(X, G) \in \mathbb{R} \cup\{+\infty\}$.

Пример 1.15. Пусть $X$ - неособая поверхность дель Пеццо такая, что $K_{X}^{2}=5$. Тогда имеется изоморфизм $\operatorname{Aut}(X) \cong \mathrm{S}_{5}\left(\right.$ см. [19]) и $\operatorname{lct}\left(X, \mathrm{~S}_{5}\right)=$ $\operatorname{lct}\left(X, \mathrm{~A}_{5}\right)=2$ (см. [14]).

ПримеР 1.16. Пусть $X$ - кубическая поверхность в $\mathbb{P}^{3}$, которая задана уравнением

$$
x^{3}+y^{3}+z^{3}+t^{3}=0 \subset \mathbb{P}^{3} \cong \operatorname{Proj}(\mathbb{C}[x, y, z, t]),
$$

и пусть $G=\operatorname{Aut}(X) \cong \mathbb{Z}_{3}^{3} \rtimes \mathrm{S}_{4}$. Тогда $\operatorname{lct}(X, G)=4$ (см. [14]).

\footnotetext{
${ }^{2}$ Рациональность числа lct $(X)$ не установлена даже в случае, когда $X$ - поверхность дель Пеццо с факторособенностями.
} 
В работах [3], [20], [21] доказан следующий результат (ср. приложение А).

ТеОрема 1.17. Предположим, что многообразие Фано X имеет факторособенности и группа $G$ компактна. Если

$$
\operatorname{lct}(X, G)>\frac{\operatorname{dim} X}{\operatorname{dim} X+1},
$$

то многообразие X допускает орбифолдную метрику Кэлера-Эйнштейна.

Теорема 1.17 имеет многочисленные применения (см. работу [20] и примеры 1.6 и 1.7).

Пример 1.18. Пусть $X$ - многообразие Фано, полученное раздутием $\mathbb{P}^{3}$ в несвязном объединении двух прямых. В работе [20] показано, что $\operatorname{lct}(X, G) \geqslant 1$, где $G$ - максимальная компактная подгруппа в $\operatorname{Aut}(X)$. По теореме 1.46 выполнено равенство $\operatorname{lct}(X)=1 / 3$.

Если многообразие, имеющее не более чем факторособенности, допускает орбифолдную метрику Кэлера-Эйнштейна, то существует три возможности: либо его канонический дивизор численно тривиален, либо его канонический дивизор обилен, либо его антиканонический дивизор обилен (многообразие Фано). На многообразии с факторособенностями, чей канонический класс численно тривиален или обилен, существует метрика Кэлера-Эйнштейна (см. [22]-[24]).

Известно несколько препятствий для существования метрики Кэлера-Эйнштейна на многообразии Фано $X$. Например, если многообразие Фано $X$ неособо, то $X$ не обладает метрикой Кэлера-Эйнштейна в случае, когда выполнено хотя бы одно из следующих условий:

- группа $\operatorname{Aut}(X)$ не является редуктивной (см. [25]);

- касательное расслоение многообразия $X$ не является прямой суммой расслоений, стабильных относительно антиканонического дивизора $-K_{X}$ (см. [26]);

- характер Футаки голоморфных векторных полей на $X$ не обращается в нуль тождественно (см. [27]).

ПримеР 1.19. Метрика Кэлера-Эйнштейна не существует на следующих многообразиях: раздутие $\mathbb{P}^{2}$ в одной или двух различных точках (см. [25]); неособое трехмерное многообразие Фано $\mathbb{P}\left(\mathscr{O}_{\mathbb{P}^{2}} \oplus \mathscr{O}_{\mathbb{P}^{2}}(1)\right)$ (см. [28]), неособое четырехмерное многообразие Фано $\mathbb{P}\left(\alpha^{*}\left(\mathscr{O}_{\mathbb{P}^{1}}(1)\right) \oplus \beta^{*}\left(\mathscr{O}_{\mathbb{P}^{2}}(1)\right)\right)$ (см. [27]), где $\alpha: \mathbb{P}^{1} \times \mathbb{P}^{2} \rightarrow \mathbb{P}^{1}$ и $\beta: \mathbb{P}^{1} \times \mathbb{P}^{2} \rightarrow \mathbb{P}^{2}-$ проекции.

Проблема существования метрик Кэлера-Эйнштейна на неособых торических многообразиях Фано решена. Имеет место следующий результат (см. [29]-[32]).

Теорема 1.20. Пусть $X$ - неособое торическое многообразие Фано. Тогда следующие условия эквивалентны:

(a) многообразие Фано $X$ обладает метрикой Кэлера-Эйнштейна;

(b) инвариант Футаки голоморфных векторных полей на $X$ обращается в нуль;

(с) барицентр рефлексивного многогранника многообразия X есть начало координат. 
Отметим, что утверждение теоремы 1.17 дает только достаточное условие для существования метрики Кэлера-Эйнштейна на многообразии Фано $X$.

ПримеР 1.21. Пусть $X$ - общая кубическая поверхность в $\mathbb{P}^{3}$, которая обладает одной точкой Эккарда (см. определение 3.1). Тогда, согласно работе [14], $\operatorname{lct}(X, \operatorname{Aut}(X))=2 / 3$, где $\operatorname{Aut}(X) \cong \mathbb{Z}_{2}$ (см. [19]). Однако каждая неособая поверхность дель Пеццо, имеющая редуктивную группу автоморфизмов, обладает метрикой Кэлера-Эйнштейна (см. [33]).

ПримеР 1.22. Пусть $X$ - общая гиперповерхность в $\mathbb{P}\left(1^{5}, 3\right)$ степени 6 . Тогда выполнено равенство $\operatorname{lct}(X, \operatorname{Aut}(X))=1 / 2($ см. пример 1.9$)$, где $\operatorname{Aut}(X) \cong \mathbb{Z}_{2}$ (см. [34]), но многообразие $X$ обладает метрикой Кэлера-Эйнштейна (см. [35]).

Числа $\operatorname{lct}(X)$ и $\operatorname{lct}(X, G)$ играют важную роль в бирациональной геометрии.

ПримеР 1.23. Предположим, что существует коммутативная диаграмма

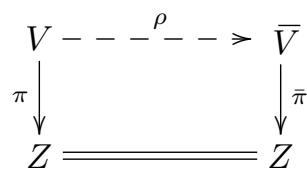

такая, что $V$ и $\bar{V}$ - многообразия, имеющие не более чем терминальные и $\mathbb{Q}$-факториальные особенности, $Z$ - неособая кривая, $\pi$ и $\bar{\pi}$ - плоские морфизмы, а $\rho$ - бирациональное отображение, которое индуцирует изоморфизм $V \backslash X \cong \bar{V} \backslash \bar{X}$, где $X$ и $\bar{X}$ - схемные слои над точкой $O \in Z$ морфизмов $\pi$ и $\bar{\pi}$ соответственно. Предположим, что схемные слои $X$ и $\bar{X}$ неприводимы и приведены, дивизоры $-K_{V}$ и $-K_{\bar{V}}$ являются $\pi$-обильным и $\bar{\pi}$-обильным соответственно, многообразия $X$ и $\bar{X}$ имеют не более чем лог-терминальные особенности, а $\rho$ не является изоморфизмом. Тогда из работ [36] и [17] следует, что

$$
\operatorname{lct}(X)+\operatorname{lct}(\bar{X}) \leqslant 1,
$$

где $X$ и $\bar{X}$ являются многообразиями Фано по формуле присоединения.

В общем случае неравенство (1.1) нельзя усилить.

ПримеР 1.24. Пусть $\pi: V \rightarrow Z$ - сюръективный плоский морфизм неособого трехмерного многообразия $V$ на неособую кривую $Z$ такой, что дивизор $-K_{V}$ является $\pi$-обильным, и пусть $X$ - схемный слой морфизма $\pi$ над точкой $O \in Z$. Предположим, что $X$ - неособая кубическая поверхность в $\mathbb{P}^{3}$, которая содержит прямые $L_{1}, L_{2}, L_{3}$, пересекающиеся в одной точке $P \in V$. В работе [37] показано, что существует коммутативная диаграмма

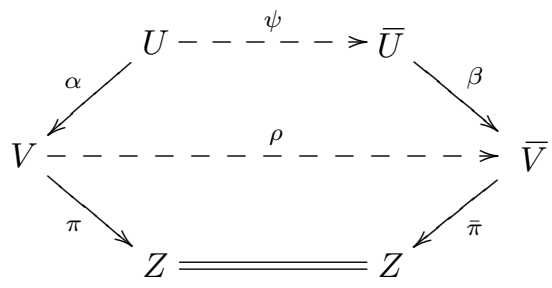


где $\alpha$ - раздутие точки $P$, отображение $\psi$ является антифлипом в собственных прообразах прямых $L_{1}, L_{2}, L_{3}$, а $\beta$ - стягивание собственного прообраза поверхности $X$. Тогда бирациональное отображение $\rho$ не является изоморфизмом, многообразие $\bar{V}$ имеет терминальные и $\mathbb{Q}$-факториальные особенности, дивизор $-K_{\bar{V}}$ является $\bar{\pi}$-обильным дивизором Картье, отображение $\rho$ индуцирует изоморфизм $V \backslash X \cong \bar{V} \backslash \bar{X}$, где $\bar{X}$ - схемный слой морфизма $\bar{\pi}$ над точкой $O$. Тогда $\bar{X}$ - кубическая поверхность с одной особой точкой типа $\mathbb{D}_{4}$, откуда $\operatorname{lct}(X)=2 / 3$ и $\operatorname{lct}(\bar{X})=1 / 3$ (см. примеры 1.10 и 1.11).

С помощью глобальных лог-канонических порогов можно доказывать нерациональность некоторых многомерных многообразий Фано.

ОПРЕДЕЛЕНиЕ 1.25. Многообразие Фано $X$ бирационально сверхжестко, если выполнено следующее:

(i) $\operatorname{rk} \operatorname{Pic}(X)=1$;

(ii) многообразие $X$ имеет $\mathbb{Q}$-факториальные и терминальные особенности;

(iii) не существует доминантного рационального отображения $\rho: X \rightarrow Y$ с рационально связными слоями такого, что $0 \neq \operatorname{dim} Y<\operatorname{dim} X$;

(iv) не существует бирационального отображения $\rho: X \rightarrow Y$ такого, что $Y$ имеет терминальные $\mathbb{Q}$-факториальные особенности и $\operatorname{rk} \operatorname{Pic}(Y)=1$;

(v) группы $\operatorname{Bir}(X)$ и $\operatorname{Aut}(X)$ совпадают.

Следующий результат известен как неравенство Нётера-Фано (см. [38]).

ТеОрема 1.26. Многообразие $X$ бирачионально сверхжестко тогда и только тогда, когда выполнено равенство $\operatorname{rk} \operatorname{Pic}(X)=1$, многообразие $X$ имеет $\mathbb{Q}$-факториальные терминальные особенности и для каждой линейной системы $\mathscr{M}$ на многообразии $X$, не имеющей неподвижных компонент, лог-пара $(X, \mathscr{M})$ канонична, где $K_{X}+\lambda \mathscr{M} \equiv 0$.

ДокАзАтельство. Одна часть требуемого утверждения хорошо известна (см. [38]), поэтому мы докажем только другую половину. Предположим, что $X$ бирационально сверхжестко и при этом на $X$ существует линейная система $\mathscr{M}$ такая, что $\mathscr{M}$ не имеет неподвижных компонент, а особенности лог-пары $(X, \lambda \mathscr{M})$ не каноничны, где $K_{X}+\lambda \mathscr{M} \sim_{\mathbb{Q}} 0$.

Рассмотрим такой бирациональный морфизм $\pi: V \rightarrow X$, что многообразие $V$ неособо и собственный прообраз системы $\mathscr{M}$ на многообразии $V$ не имеет базисных точек. Пусть $\mathscr{B}$ - собственный прообраз системы $\mathscr{M}$ на многообразии $V$. Тогда

$$
K_{V}+\lambda \mathscr{B} \sim_{\mathbb{Q}} \pi^{*}\left(K_{X}+\lambda \mathscr{M}\right)+\sum_{i=1}^{r} a_{i} E_{i} \sim_{\mathbb{Q}} \sum_{i=1}^{r} a_{i} E_{i},
$$

где $E_{i}-$ исключительный дивизор морфизма $\pi$ и $a_{i} \in \mathbb{Q}$.

Из работы [39] следует существование коммутативной диаграммы

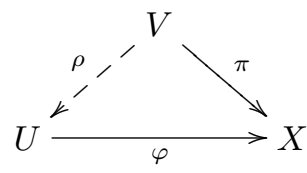


такой, что $\rho$ - бирациональное отображение, морфизм $\varphi$ бирационален, дивизор

$$
K_{U}+\lambda \rho(\mathscr{B}) \sim_{\mathbb{Q}} \varphi^{*}\left(K_{X}+\lambda \mathscr{M}\right)+\sum_{i=1}^{r} a_{i} \rho\left(E_{i}\right) \sim_{\mathbb{Q}} \sum_{i=1}^{r} a_{i} \rho\left(E_{i}\right)
$$

численно эффективен относительно морфизма $\varphi$, многообразие $U$ имеет $\mathbb{Q}$-факториальные особенности, а лог-пара $(U, \lambda \rho(\mathscr{B}))$ имеет терминальные особенности.

Отметим, что $\varphi$ не является изоморфизмом. Действительно, из [40; п. 1.1] следует, что

$$
a_{i}>0 \Longrightarrow \operatorname{dim}\left(\rho\left(E_{i}\right)\right) \leqslant \operatorname{dim} X-2,
$$

а поскольку особенности лог-пары $(X, \lambda \mathscr{M})$ не являются каноническими по предположению, из конструкции отображения $\rho$ следует существование $k \in$ $\{1, \ldots, r\}$ такого, что выполнено неравенство $a_{k}<0$ и подмногообразие $\rho\left(E_{k}\right)$ является дивизором в $U$.

Таким образом, мы видим, что дивизор $K_{U}+\lambda \rho(\mathscr{B})$ не является псевдоэффективным. В этом случае из работы [39] следует существование диаграммы

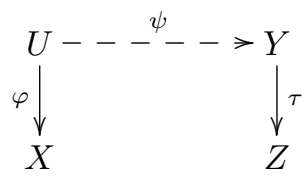

такой, что $\psi$ - бирациональное отображение, морфизм $\tau$ является Мори расслоенным пространством (см. [41]), а дивизор $-\left(K_{Y}+\lambda(\psi \circ \rho)(\mathscr{B})\right)$ обилен относительно морфизма $\tau$.

Многообразие $Y$ имеет терминальные и $\mathbb{Q}$-факториальные особенности, и выполнено равенство $\mathrm{rk} \operatorname{Pic}(Y / Z)=1$. Тогда отображение $\psi \circ \rho \circ \pi^{-1}$ не является изоморфизмом, поскольку $K_{X}+\lambda \mathscr{M} \sim_{\mathbb{Q}} 0$, но общий слой морфизма $\tau$ рационально связен (см. [4]), что противоречит сделанному ранее предположению о том, что многообразие $X$ является бирационально сверхжестким. Утверждение теоремы доказано.

Бирационально сверхжесткие многообразия Фано нерациональны (см. [38]), откуда следует, что $\operatorname{dim}(X) \neq 2$, если $X$ бирационально сверхжестко (ср. [42]).

ПримеР 1.27. Общая гиперповерхность в $\mathbb{P}^{n}$ степени $n \geqslant 4$ и в $\mathbb{P}\left(1^{n+1}, n\right)$ степени $2 n \geqslant 6$ бирационально сверхжестка (см. [43], [7]).

Следующий результат доказан в работе [7].

Теорема 1.28. Пусть $X_{1}, \ldots, X_{r}$ - бирационально сверхжесткие многообразия Фано такие, что выполнены неравенства $\operatorname{lct}\left(X_{i}\right) \geqslant 1, i=1, \ldots, r$. Тогда

(а) многообразие $X_{1} \times \cdots \times X_{r}$ нерационально и

$$
\operatorname{Bir}\left(X_{1} \times \cdots \times X_{r}\right)=\operatorname{Aut}\left(X_{1} \times \cdots \times X_{r}\right)
$$


(b) для каждого рачионального доминантного отображения $\rho: X_{1} \times \cdots \times$ $X_{r} \rightarrow Y$, чей общий слой является рационально связным многообразием, существует коммутативная диаграмма

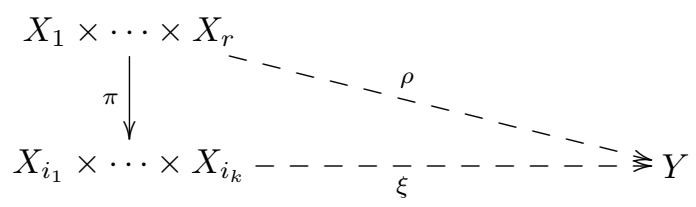

для некоторого подмножества $\left\{i_{1}, \ldots, i_{k}\right\} \subseteq\{1, \ldots, r\}$, где $\xi$ - бирациональное отображение, а $\pi$ - проекиия.

Из примеров 1.6 и 1.27 следует, что многообразия, удовлетворяющие всем условиям теоремы 1.28 , действительно существуют. Примеры таких многообразий можно строить явно.

ПримеР 1.29. Пусть $X$ - гиперповерхность, заданная уравнением

$$
w^{2}=x^{6}+y^{6}+z^{6}+t^{6}+x^{2} y^{2} z t \subset \mathbb{P}(1,1,1,1,3) \cong \operatorname{Proj}(\mathbb{C}[x, y, z, t, w]),
$$

где $\operatorname{wt}(x)=\operatorname{wt}(y)=\operatorname{wt}(z)=\operatorname{wt}(t)=1$ и $\operatorname{wt}(w)=3$. Тогда $X$ является неособым и бирационально сверхжестким (см. [44]), а из работы [8] следует, что $\operatorname{lct}(X)=1$.

Предположим дополнительно, что подгруппа $G \subset \operatorname{Aut}(X)$ конечна.

ОПРЕДЕЛЕНИЕ 1.30. Многообразие Фано $X$ называется $G$-бирационально сверхжестким, если выполнены следующие условия:

- $G$-инвариантная подгруппа группы $\mathrm{Cl}(X)$ изоморфна группе $\mathbb{Z}$;

- многообразие $X$ имеет терминальные особенности;

- не существует такого доминантного $G$-эквивариантного рационального отображения $\rho: X \rightarrow Y$ с рационально связными слоями, что $0 \neq$ $\operatorname{dim} Y<\operatorname{dim} X$

- не существует такого небирегулярного $G$-эквивариантного бирационального отображения $\rho: X \rightarrow Y$, что многообразие $Y$ имеет не более чем терминальные особенности, а $G$-инвариантная подгруппа группы $\mathrm{Cl}(Y)$ изоморфна группе $\mathbb{Z}$.

Из доказательства теоремы 1.26 вытекает следующий результат.

Теорема 1.31. Многообразие $X$ является $G$-бирационально сверхжестким, если и только если $G$-инвариантная подгруппа группљ $\mathrm{Cl}(X)$ изоморфна группе $\mathbb{Z}$, многообразие $X$ имеет терминальные особенности и для каждой $G$-инвариантной линейной системы $\mathscr{M}$ на многообразии $X$, которая не имеет неподвижных компонент, лог-пара $(X, \lambda \mathscr{M})$ имеет канонические особенности, где $K_{X}+\lambda \mathscr{M} \sim_{\mathbb{Q}} 0$.

Из доказательства теоремы 1.28 вытекает следующий результат (см. [14]).

Теорема 1.32. Пусть $X_{i}-$ многообразие Фано, и пусть $G_{i} \subset \operatorname{Aut}\left(X_{i}\right)-$ конечная подгруппа такая, что многообразие $X_{i}$ является $G_{i}$-бирационально сверхжестким и выполнено неравенство $\operatorname{lct}\left(X_{i}, G_{i}\right) \geqslant 1$ при $i=1, \ldots, r$. Тогда 
(а) многообразие $X_{1} \times \cdots \times X_{r}$ не обладает $G_{1} \times \cdots \times G_{r}$-эквивариантным бирациональным отображением $\rho: X_{1} \times \cdots \times X_{r}-\rightarrow \mathbb{P}^{n}$;

(b) каждый $G_{1} \times \cdots \times G_{r}$-эквивариантный бирациональный автоморфизм многообразия $X_{1} \times \cdots \times X_{r}$ является бирегулярным;

(c) для каждого $G_{1} \times \cdots \times G_{r}$-эквивариантного рационального доминантного отображения $\rho: X_{1} \times \cdots \times X_{r} \rightarrow Y$, чей общий слой является рационально связным многообразием, существует коммутативная диаграмма

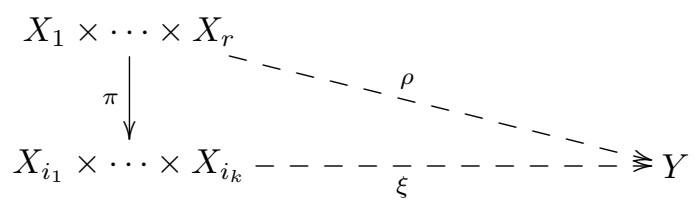

для некоторого подмножества $\left\{i_{1}, \ldots, i_{k}\right\} \subseteq\{1, \ldots, r\}$, где $\xi$ является бираицональным отображением, а $\pi$ является естественной проекцией.

Многообразия, удовлетворяющие условиям теоремы 1.32 , существуют (см. пример 1.16).

ПримеР 1.33. Простая группа $\mathrm{A}_{6}$ является группой автоморфизмов кривой

$$
10 x^{3} y^{3}+9 z x^{5}+9 z y^{5}+27 z^{6}=45 x^{2} y^{2} z^{2}+135 x y z^{4} \subset \mathbb{P}^{2} \cong \operatorname{Proj}(\mathbb{C}[x, y, z]),
$$

что индуцирует вложение $\mathrm{A}_{6} \subset \operatorname{Aut}\left(\mathbb{P}^{2}\right)$. Тогда $\mathbb{P}^{2}$ является $\mathrm{A}_{6}$-бирационально сверхжесткой поверхностью такой, что $\operatorname{lct}\left(\mathbb{P}^{2}, \mathrm{~A}_{6}\right)=2$ (см. [14]). По теореме 1.32 существует индуцированное вложение $\mathrm{A}_{6} \times \mathrm{A}_{6} \cong \Omega \subset \operatorname{Bir}\left(\mathbb{P}^{4}\right)$ такое, что $\Omega$ не сопряжена подгруппам в $\operatorname{Aut}\left(\mathbb{P}^{4}\right)$.

Теперь мы рассмотрим многообразия Фано, чья бирациональная геометрия очень похожа на бирациональную геометрию бирационально сверхжестких многообразий Фано.

ОПРЕДЕЛЕНИЕ 1.34. Многообразие $X$ бирационально жестко, если:

- выполнено равенство $\operatorname{rk} \operatorname{Pic}(X)=1$;

- многообразие $X$ имеет $\mathbb{Q}$-факториальные и терминальные особенности;

- не существует рационального отображения $\rho: X \rightarrow Y$ с рационально связными слоями такого, что $0 \neq \operatorname{dim} Y<\operatorname{dim} X$;

- не существует бирационального отображения $\rho: X \rightarrow Y$ такого, что $Y \neq X$, многообразие $Y$ имеет $\mathbb{Q}$-факториальные терминальные особенности и $\operatorname{rk} \operatorname{Pic}(Y)=1$.

Из доказательства теоремы 1.26 вытекает следующий результат.

Теорема 1.35. Многообразие X бирачионалъно жестко, если и только если $\operatorname{rk} \operatorname{Pic}(X)=1$, многообразие $X$ имеет $\mathbb{Q}$-факториальные терминальнье особенности и для каждой непустой линейной системы $\mathscr{M}$ на многообразии $X$, не имеющей неподвижных компонент, существует $\xi \in \operatorname{Bir}(X)$ такой, что лог-пара $(X, \lambda \xi(\mathscr{M}))$ канонична, где $K_{X}+\lambda \xi(\mathscr{M}) \equiv 0$.

Бирационально жесткие многообразия Фано нерациональны (см. [38]). 
ОПРЕДЕЛЕНИЕ 1.36. Пусть $X$ бирационально жестко. Подмножество $Г \subset$ $\operatorname{Bir}(X)$ откручивает все максимальные особенности, если для каждой линейной системы $\mathscr{M}$ на многообразии $X$, не имеющей неподвижных компонент, существует $\xi \in \Gamma$ такое, что лог-пара $(X, \lambda \xi(\mathscr{M}))$ канонична, где $\lambda$ - такое рациональное число, что $K_{X}+\lambda \xi(\mathscr{M}) \equiv 0$.

Из бирациональной жесткости многообразия $X$ и существования подмножества $\Gamma \subset \operatorname{Bir}(X)$, которое откручивает все максимальные особенности, следует, что $\operatorname{Bir}(X)=\langle\Gamma, \operatorname{Aut}(X)\rangle$.

ОПРЕДЕЛЕНИЕ 1.37. Многообразие $X$ называется универсально бирационально жестким, если для любого многообразия $U$ многообразие $X \otimes$ $\operatorname{Spec}(\mathbb{C}(U))$ бирационально жестко над полем рациональных функций $\mathbb{C}(U)$ многообразия $U$.

Отметим, что определение 1.34 обобщается на многообразия Фано, определенные над произвольным совершенным полем (см. [42], [19]).

ПримеР 1.38. Пусть $X$ - трехмерное многообразие такое, что существует конечный морфизм $\pi: X \rightarrow Q \subset \mathbb{P}^{3}$, где $Q$ - неособая трехмерная квадрика, а $\pi$ - двойное накрытие с ветвлением в неособой поверхности $S \subset Q$ степени 8. Существует однопараметрическое семейство кривых

$$
\mathscr{C}=\left\{C \subset X \mid C-\text { неособая кривая такая, что }-K_{X} \cdot C=1\right\},
$$

и для каждой кривой $C \in \mathscr{C}$ существует коммутативная диаграмма

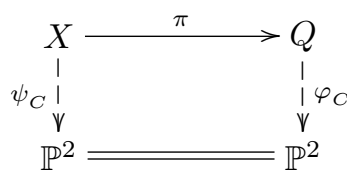

где $\varphi_{C}-$ проекция из прямой $\pi(C)$. Тогда общий слой отображения $\psi_{C}-$ неособая эллиптическая кривая. Рациональное отображение $\psi_{C}$ задает эллиптическое расслоение с сечением, которое индуцирует бирациональную инволюцию $\tau_{C}$. Известно, что

$$
\psi_{C} \in \operatorname{Aut}(X) \Longleftrightarrow C \subset S
$$

и в случае, когда многообразие $X$ достаточно общее, поверхность $S$ не содержит кривых из семейства $\mathscr{C}$. В работе [44] показано, что существует точная последовательность групп

$$
1 \rightarrow \Gamma \rightarrow \operatorname{Bir}(X) \rightarrow \operatorname{Aut}(X) \rightarrow 1
$$

где $\Gamma$ - свободное произведением подгрупп, порожденных бирациональными небирегулярными инволюциями $\tau_{C}, C \in \mathscr{C}$. Тогда $X$ универсально бирационально жестко.

Бирационально сверхжесткие многообразия Фано универсально бирационально жестки. 
ОПРЕДЕЛЕНИЕ 1.39. Предположим, что $X$ универсально бирационально жестко. Подмножество $\Gamma \subset \operatorname{Bir}(X)$ универсально откручивает все максимальные особенности, если для любого многообразия $U$ индуцированное подмножество

$$
\Gamma \subset \operatorname{Bir}(X) \subseteq \operatorname{Bir}(X \otimes \operatorname{Spec}(\mathbb{C}(U)))
$$

откручивает все максимальные особенности на многообразии $X \otimes \operatorname{Spec}(\mathbb{C}(U))$.

Легко видеть, что любое подмножество в $\operatorname{Aut}(X)$ универсально откручивает все максимальные особенности, если многообразие Фано $X$ является бирационально сверхжестким.

ЗАмечаниЕ 1.40. Пусть $X$ - бирационально жесткое многообразие Фано. Рассмотрим произвольное подмножество $\Gamma \subseteq \operatorname{Bir}(X)$ и предположим, что $\operatorname{dim} X \neq 1$. Тогда из работы [45] следует, что следующие условия эквивалентны:

- подмножество Г универсально откручивает все максимальные особенности;

- подмножество Г откручивает все максимальные особенности, а группа $\operatorname{Bir}(X)$ счетна.

ПримеР 1.41. В обозначениях и предположениях примера 1.7 предположим, что гиперповерхность $X$ общая. Тогда:

- гиперповерхность $X$ является универсально бирационально жесткой (см. [46]),

- существуют инволюции $\tau_{1}, \ldots, \tau_{k} \in \operatorname{Bir}(X)$ такие, что последовательность групп

$$
1 \rightarrow\left\langle\tau_{1}, \ldots, \tau_{k}\right\rangle \rightarrow \operatorname{Bir}(X) \rightarrow \operatorname{Aut}(X) \rightarrow 1
$$

точна (см. [46], [47]), где $\left\langle\tau_{1}, \ldots, \tau_{k}\right\rangle$ - подгруппа, порожденная $\tau_{1}, \ldots, \tau_{k}$,

- $\left\langle\tau_{1}, \ldots, \tau_{k}\right\rangle$ универсально откручивает все максимальные особенности (cM. [46]),

а все соотношения между инволюциями $\tau_{1}, \ldots, \tau_{k}$ найдены в работе [47].

Пусть $X_{1}, \ldots, X_{r}$ - многообразия Фано с $\mathbb{Q}$-факториальными терминальными особенностями такие, что $\operatorname{rk} \operatorname{Pic}\left(X_{1}\right)=\cdots=\operatorname{rk} \operatorname{Pic}\left(X_{r}\right)=1$. Рассмотрим проекцию

$\pi_{i}: X_{1} \times \cdots \times X_{i-1} \times X_{i} \times X_{i+1} \times \cdots \times X_{r} \rightarrow X_{1} \times \cdots \times X_{i-1} \times \widehat{X_{i}} \times X_{i+1} \times \cdots \times X_{r}$

и обозначим символом $\mathscr{X}_{i}$ общий слой проекции $\pi_{i}$ в схемном смысле; многообразие $\mathscr{X}_{i}$ определено над полем $\mathbb{C}\left(X_{1} \times \cdots \times X_{i-1} \times \widehat{X}_{i} \times X_{i+1} \times \cdots \times X_{r}\right)$.

ЗАмечАниЕ 1.42. Существуют естественные вложения групп

$$
\prod_{i=1}^{r} \operatorname{Bir}\left(X_{i}\right) \subseteq\left\langle\operatorname{Bir}\left(\mathscr{X}_{1}\right), \ldots, \operatorname{Bir}\left(\mathscr{X}_{r}\right)\right\rangle \subseteq \operatorname{Bir}\left(X_{1} \times \cdots \times X_{r}\right)
$$

В работе [11] доказано следующее обобщение теоремы 1.28. 
Теорема 1.43. Предположим, что $X_{1}, \ldots, X_{r}$ универсально бирачионально жестки и выполнены неравенства $\operatorname{lct}\left(X_{i}\right) \geqslant 1, i=1, \ldots, r$. Тогда

(а) многообразие $X_{1} \times \cdots \times X_{r}$ нерационально и

$$
\operatorname{Bir}\left(X_{1} \times \cdots \times X_{r}\right)=\left\langle\operatorname{Bir}\left(\mathscr{X}_{1}\right), \ldots, \operatorname{Bir}\left(\mathscr{X}_{r}\right), \operatorname{Aut}\left(X_{1} \times \cdots \times X_{r}\right)\right\rangle,
$$

(b) для любого рационального доминантного отображения $\rho: X_{1} \times \cdots \times$ $X_{r} \rightarrow Y$ такого, что общий слой отображения $\rho$ рационально связен, существуют подмножество $\left\{i_{1}, \ldots, i_{k}\right\} \subseteq\{1, \ldots, r\}$ и коммутативная диаграмма

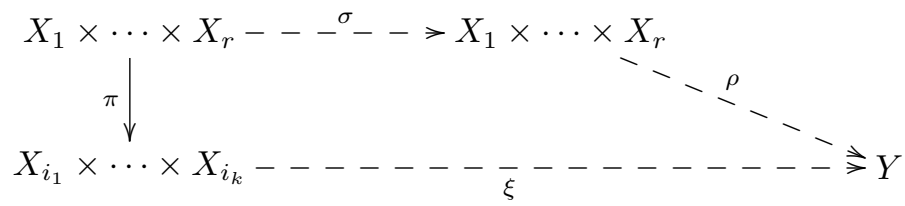

где $\pi$-естественная проекиия, а $\xi$ и $\sigma$-бирациональные отображения.

СледСТВИЕ 1.44. Предположим, что существует подгруппа $\Gamma_{i} \subseteq \operatorname{Bir}\left(X_{i}\right)$, универсально откручивающая все максимальные особенности, $u \operatorname{lct}\left(X_{i}\right) \geqslant 1$ для всех $i=1, \ldots, r$. Тогда

$$
\operatorname{Bir}\left(X_{1} \times \cdots \times X_{r}\right)=\left\langle\prod_{i=1}^{r} \Gamma_{i}, \operatorname{Aut}\left(X_{1} \times \cdots \times X_{r}\right)\right\rangle .
$$

В частности, с помощью примеров 1.7 и 1.41 мы получаем следующий пример.

ПРИмеР 1.45 (ср. с примером 1.41). Пусть $X$ - общая гиперповерхность в $\mathbb{P}(1,1,4,5,10)$ степени 20 . Тогда существует точная последовательность групп

$$
1 \rightarrow \prod_{i=1}^{m}\left(\mathbb{Z}_{2} * \mathbb{Z}_{2}\right) \rightarrow \operatorname{Bir}(\underbrace{X \times \cdots \times X}_{m \text { сомножителей }}) \rightarrow \mathrm{S}_{m} \rightarrow 1,
$$

где $\mathrm{S}_{m}$ - группа перестановок, а $\mathbb{Z}_{2} * \mathbb{Z}_{2}$ - бесконечная группа диэдра.

Предположим, что $X$ является неособым трехмерным многообразием Фано (см. [2]). Неособое трехмерное многообразие Фано $X$ принадлежит одному из 105 деформационных семейств (см. [48]-[52]). Обозначим символом

$$
J(X) \in\{1.1,1.2, \ldots, 1.17,2.1, \ldots, 2.36,3.1, \ldots, 3.31,4.1, \ldots, 4.13,5.1, \ldots, 5.8\}
$$

порядковый номер деформационного типа многообразия $X$ в обозначениях таблицы 1 (см. приложение В). Основная цель настоящей работы - доказать следующий результат.

ТеОРема 1.46. Выполнены следующие утверждения:

(a) $\operatorname{lct}(X)=1 / 5$, если $\beth(X) \in\{2.36,3.29\}$;

(b) $\operatorname{lct}(X)=1 / 4$, если $\mathrm{I}(X) \in\{1.17,2.28,2.30,2.33,2.35,3.23,3.26,3.30,4.12\}$;

(c) $\operatorname{lct}(X)=1 / 3$, если $I(X) \in\{1.16,2.29,2.31,2.34,3.9,3.18,3.19,3.20,3.21$, $3.22,3.24,3.25,3.28,3.31,4.4,4.8,4.9,4.10,4.11,5.1,5.2\}$; 
(d) $\operatorname{lct}(X)=3 / 7$, если $\mathrm{I}(X)=4.5$;

(e) $\operatorname{lct}(X)=1 / 2$, если $\exists(X) \in\{1.11,1.12,1.13,1.14,1.15,2.1,2.3,2.18,2.25,2.27$, $2.32,3.4,3.10,3.11,3.12,3.14,3.15,3.16,3.17,3.24,3.27,4.1,4.2,4.3,4.6$, 4.7, 5.3, 5.4, $5.5,5.6,5.7,5.8\}$

(f) если многообразие $X$ является общим в своем деформачионном классе, mo

$$
\begin{aligned}
& -\operatorname{lct}(X)=1 / 3, \text { если } \beth(X)=2.23 \text {, } \\
& \text { - } \operatorname{lct}(X)=1 / 2 \text {, если }\rfloor(X) \in\{2.5,2.8,2.10,2.11,2.14,2.15,2.19,2.24,2.26,3.2 \text {, } \\
& \text { 3.5, 3.6, 3.7, 3.8, 4.13\}, } \\
& \text { - } \operatorname{lct}(X)=2 / 3 \text {, если }\rfloor(X)=3.3 \text {, } \\
& -\operatorname{lct}(X)=3 / 4, \text { если } \beth(X) \in\{2.4,3.1\} \text {, } \\
& -\operatorname{lct}(X)=1 \text {, если } \Xi(X)=1.1 \text {. }
\end{aligned}
$$

В общем случае условие общности в теореме 1.46 существенно.

ПримеР 1.47. Предположим, что $I(X)=4.13$. Отметим, что данный деформационный класс был по ошибке пропущен в работе [50], но он был найден позднее в работе [51]. Существует бирациональный морфизм $\alpha: X \rightarrow$ $\mathbb{P}^{1} \times \mathbb{P}^{1} \times \mathbb{P}^{1}$ такой, что $\alpha$ стягивает некоторую неособую и неприводимую поверхность $E \subset X$ на кривую $C$ такую, что $C \cdot F_{1}=C \cdot F_{2}=1$ и $C \cdot F_{3}=3$, где $F_{1} \cong F_{2} \cong F_{3} \cong \mathbb{P}^{1} \times \mathbb{P}^{1}$ являются слоями трех различных естественных проекций $\mathbb{P}^{1} \times \mathbb{P}^{1} \times \mathbb{P}^{1} \rightarrow \mathbb{P}^{1}$. Из теоремы 1.46 следует, что $\operatorname{lct}(X)=1 / 2$ в случае, когда $X$ является общим. Отметим, что существует единственная поверхность $G \in\left|F_{1}+F_{2}\right|$ такая, что $C \subset G$. Тогда $-K_{X} \sim 2 \bar{G}+E+\bar{F}_{3}$, где $\bar{F}_{3} \subset X \supset \bar{G}$ - собственные прообразы поверхностей $F_{3}$ и $G$ соответственно. При этом $\operatorname{lct}(X) \leqslant 1 / 2$, но $\operatorname{lct}(X) \leqslant \operatorname{lct}\left(X, 2 \bar{G}+E+\bar{F}_{3}\right) \leqslant 4 / 9<1 / 2$ в случае, когда $\left|F_{3} \cap C\right|=1$.

Настоящая работа организована следующим образом. В разделах 2,3 и 4 мы рассматриваем вспомогательные результаты, которые будут использованы при доказательстве теоремы 1.46. В разделе 5 мы находим глобальный лог-канонический порог торических многообразий Фано. В разделе 6 мы доказываем теорему 1.46 для неособых трехмерных многообразий Фано индекса 2 , т. е. для $I(X) \in\{1.11,1.12,1.13,1.14,1.15,2.32,2.35,3.27\}$. В разделе 7 мы доказываем теорему 1.46 в случае, когда $\operatorname{rk} \operatorname{Pic}(X)=2$. В разделе 8 мы доказываем теорему 1.46 в случае, когда $\operatorname{rk} \operatorname{Pic}(X)=3$. В разделе 9 мы доказываем теорему 1.46 в случае, когда $\operatorname{rk} \operatorname{Pic}(X) \geqslant 4$. В разделе 10 мы находим верхние оценки для числа $\operatorname{lct}(X)$ в случае, когда

$$
J(X) \in\{1.8,1.9,1.10,2.2,2.7,2.9,2.12,2.13,2.16,2.17,2.20,2.21,2.22,3.13\} .
$$

В приложении А, которое написано Ж.-П. Демайи, показана связь между глобальными лог-каноническими порогами неособых многообразий Фано и $\alpha$-инвариантами неособых многообразий Фано, которые были введены в работе [3] для изучения вопроса существования метрик Кэлера-Эйнштейна на неособых многообразиях Фано. В приложении В мы приводим таблицу 1, которая содержит список всех неособых трехмерных многообразий Фано вместе с известными значениями и оценками их глобальных лог-канонических порогов. 
Мы будем использовать стандартное обозначение $D_{1} \sim D_{2}$ (соответственно $D_{1} \sim_{\mathbb{Q}} D_{2}$ ) для линейно эквивалентных (соответственно $\mathbb{Q}$-линейно эквивалентных) дивизоров (соответственно $\mathbb{Q}$-дивизоров). Если дивизор (соответственно $\mathbb{Q}$-дивизор) $D$ является линейно эквивалентным некоторому линейному расслоению $\mathscr{L}$ (соответственно является $\mathbb{Q}$-линейно эквивалентным дивизору, который линейно эквивалентен линейному расслоению $\mathscr{L})$, то мы используем обозначение $D \sim \mathscr{L}$ (соответственно $D \sim \mathbb{Q} \mathscr{L})$. Отметим, что $\mathbb{Q}$-линейная эквивалентность совпадает с численной эквивалентностью для многообразий Фано, имеющих лог-терминальные особенности. Под проективизацией $\mathbb{P}_{Y}(\mathscr{E})$ векторного расслоения $\mathscr{E}$ на многообразии $Y$ подразумевается многообразие гиперплоскостей в слоях $\mathscr{E}$. Символ $\mathbb{F}_{n}$ обозначает поверхность Хирцебруха $\mathbb{P}\left(\mathscr{O}_{\mathbb{P}^{1}} \oplus \mathscr{O}_{\mathbb{P}^{1}}(n)\right)$. Мы всегда рассматриваем неособое трехмерное многообразие Фано $X$ в совокупности с порядковым номером $I(X)$ соответствующего деформационного класса, который введен в таблице 1.

Мы очень признательны Ж.-П. Демайи за приложение А, а также А. Илиеву, А.Г. Кузнецову и Ю.Г. Прохорову за полезные обсуждения. Мы благодарны Институту высших научных исследований (Бюр-сюр-Иветт, Франция) и Математическому институту им. Макса Планка (Бонн, Германия) за гостеприимство.

\section{2. Предварительные сведения}

Пусть $X$ - многообразие, имеющее не более чем лог-терминальные особенности. Рассмотрим дивизор $B_{X}=\sum_{i=1}^{r} a_{i} B_{i}$, где $B_{i}-$ неприводимый дивизор Вейля на многообразии $X$, а $a_{i}$ - неотрицательное рациональное число. Предположим, что $B_{X}$ является дивизором $\mathbb{Q}$-Картье и $B_{i} \neq B_{j}$ при $i \neq j$. Рассмотрим бирациональный морфизм $\pi: \bar{X} \rightarrow X$ такой, что $\bar{X}$ неособо. Положим $B_{\bar{X}}=\sum_{i=1}^{r} a_{i} \bar{B}_{i}$, где $\bar{B}_{i}-$ собственный прообраз $B_{i}$ на многообразии $\bar{X}$. Тогда

$$
K_{V}+B_{\bar{X}} \equiv \pi^{*}\left(K_{X}+B_{X}\right)+\sum_{i=1}^{n} c_{i} E_{i},
$$

где $c_{i} \in \mathbb{Q}$, а $E_{i}$ - исключительный дивизор морфизма $\pi$. Предположим дополнительно, что дивизор $\left(\bigcup_{i=1}^{r} \bar{B}_{i}\right) \cup\left(\bigcup_{i=1}^{n} E_{i}\right)$ есть дивизор с простыми нормальными пересечениями, и положим $B^{\bar{X}}=B_{\bar{X}}-\sum_{i=1}^{n} c_{i} E_{i}$.

ОПРЕДЕЛЕНИЕ 2.1. Лог-пара $\left(X, B_{X}\right)$ имеет лог-канонические (соответственно лог-терминальные) особенности, если

- выполнено $a_{i} \leqslant 1$ (соответственно $a_{i}<1$ ) для всех $i=1, \ldots, r$,

- выполнено $c_{j} \geqslant-1$ (соответственно $c_{j}>-1$ ) для всех $j=1, \ldots, n$.

Известно, что определение 2.1 не зависит от выбора морфизма $\pi: \bar{X} \rightarrow X$. Положим

$$
\operatorname{LCS}\left(X, B_{X}\right)=\left(\bigcup_{a_{i} \geqslant 1} B_{i}\right) \cup\left(\bigcup_{c_{i} \leqslant-1} \pi\left(E_{i}\right)\right) \subsetneq X
$$

и будем называть $\operatorname{LCS}\left(X, B_{X}\right)$ множеством лог-канонических особенностей логпары $\left(X, B_{X}\right)$. 
ОПРЕДЕЛЕНИЕ 2.2. Собственное неприводимое подмногообразие $Y \subsetneq X$ называется центром лог-канонических особенностей лог-пары $\left(X, B_{X}\right)$, если

- либо выполнено неравенство $a_{i} \geqslant 1$ и $Y=B_{i}$,

- либо выполнено неравенство $c_{i} \leqslant-1$ и $Y=\pi\left(E_{i}\right)$ для некоторого морфизма $\pi: \bar{X} \rightarrow X$.

Обозначим символом $\mathbb{L} \mathbb{C} S\left(X, B_{X}\right)$ множество, состоящее из всех центров логканонических особенностей лог-пары $\left(X, B_{X}\right)$. Тогда

$$
Y \in \mathbb{L} \mathbb{C S}\left(X, B_{X}\right) \Longrightarrow Y \subseteq \operatorname{LCS}\left(X, B_{X}\right)
$$

и $\mathbb{L} \mathbb{C S}\left(X, B_{X}\right)=\varnothing \Longleftrightarrow \operatorname{LCS}\left(X, B_{X}\right)=\varnothing \Longleftrightarrow$ лог-пара $\left(X, B_{X}\right)$ лог-терминальна.

ЗАмечАниЕ 2.3. Пусть $\mathscr{H}$ - линейная система на многообразии $X$, не имеющая базисных точек, пусть $H$ - достаточно общий дивизор в линейной системе $\mathscr{H}$, и пусть $Y \subsetneq X-$ неприводимое подмногообразие. Положим $\left.Y\right|_{H}=$ $\sum_{i=1}^{m} Z_{i}$, где $Z_{i} \subset H$ - неприводимое подмногообразие. Тогда из определения 2.2 следует (ср. с теоремой 2.19), что

$$
Y \in \mathbb{L} \mathbb{C} S\left(X, B_{X}\right) \Longleftrightarrow\left\{Z_{1}, \ldots, Z_{m}\right\} \subseteq \mathbb{L} \mathbb{C} \mathbb{S}\left(H,\left.B_{X}\right|_{H}\right)
$$

ПримеР 2.4. Пусть $\alpha: V \rightarrow X$ - раздутие неособой точки $O \in X$. Тогда $B_{V} \equiv \alpha^{*}\left(B_{X}\right)-\operatorname{mult}_{O}\left(B_{X}\right) E$, где $\operatorname{mult}_{O}\left(B_{X}\right) \in \mathbb{Q}$, а $E$ - исключительный дивизор раздутия $\alpha$. Тогда $\operatorname{mult}_{O}\left(B_{X}\right)>1$, если лог-пара $\left(X, B_{X}\right)$ не логканонична в точке $O$. Положим

$$
B^{V}=B_{V}+\left(\operatorname{mult}_{O}\left(B_{X}\right)-\operatorname{dim}(X)+1\right) E
$$

и предположим, что $\operatorname{mult}_{O}\left(B_{X}\right) \geqslant \operatorname{dim}(X)-1$. Тогда $O \in \mathbb{L} \mathbb{C} S\left(X, B_{X}\right)$, если и только если

- либо $E \in \mathbb{L} \mathbb{C S}\left(V, B^{V}\right)$, т. е. $\operatorname{mult}_{O}\left(B_{X}\right) \geqslant \operatorname{dim}(X)$,

- либо существует подмногообразие $Z \subsetneq E$ такое, что $Z \in \mathbb{L} \mathbb{C S}\left(V, B^{V}\right)$.

На множестве $\operatorname{LCS}\left(X, B_{X}\right)$ можно ввести структуру схемы (см. [20], [40]). Положим

$$
\mathscr{I}\left(X, B_{X}\right)=\pi_{*}\left(\sum_{i=1}^{n}\left\lceil c_{i}\right\rceil E_{i}-\sum_{i=1}^{r}\left\lfloor a_{i}\right\rfloor \bar{B}_{i}\right),
$$

и пусть $\mathscr{L}\left(X, B_{X}\right)$ - подсхема, ассоциированная с пучком идеалов $\mathscr{I}\left(X, B_{X}\right)$.

ОПРедЕЛЕНИЕ 2.5. Для данной лог-пары $\left(X, B_{X}\right)$ будем называть подсхему $\mathscr{L}\left(X, B_{X}\right)$ подсхемой лог-канонических особенностей лог-пары $\left(X, B_{X}\right)$ и пучок идеалов $\mathscr{I}\left(X, B_{X}\right)$ - пучком множительных идеалов лог-пары $\left(X, B_{X}\right)$.

Из конструкции подсхемы $\mathscr{L}\left(X, B_{X}\right)$ немедленно следует, что

$$
\operatorname{Supp}\left(\mathscr{L}\left(X, B_{X}\right)\right)=\operatorname{LCS}\left(X, B_{X}\right) \subset X .
$$

Следующий результат называется теоремой Шокурова об обращении в нуль (см. [40]) или теоремой Наделя об обращении в нуль (см. [53; теорема 9.4.8]). 
Теорема 2.6. Пусть $H$ - численно эффективный и обгемный $\mathbb{Q}$-дивизор на $X$ такой, что выполнена эквивалентность $K_{X}+B_{X}+H \equiv D$ для некоторого дивизора Kартье $D$ на многообразии $X$. Тогда для всех $i \geqslant 1$

$$
H^{i}\left(X, \mathscr{I}\left(X, B_{X}\right) \otimes D\right)=0 .
$$

Для дивизора Картье $D$ на многообразии $X$ рассмотрим точную последовательность

$$
0 \rightarrow \mathscr{I}\left(X, B_{X}\right) \otimes D \rightarrow \mathscr{O}_{X}(D) \rightarrow \mathscr{O}_{\mathscr{L}\left(X, B_{X}\right)}(D) \rightarrow 0
$$

и соответствующую точную последовательность групп когомологий

$$
H^{0}\left(\mathscr{O}_{X}(D)\right) \rightarrow H^{0}\left(\mathscr{O}_{\mathscr{L}\left(X, B_{X}\right)}(D)\right) \rightarrow H^{1}\left(\mathscr{I}\left(X, B_{X}\right) \otimes D\right)
$$

Теорема 2.7. Если $-\left(K_{X}+B_{X}\right)$ численно эбфективен и объемен, то множество $\operatorname{LCS}\left(X, B_{X}\right)$ связно.

ДокАЗАТЕЛЬСтво. Положим $D=0$. Тогда из теоремы 2.6 следует, что последовательность

$$
\mathbb{C}=H^{0}\left(\mathscr{O}_{X}\right) \rightarrow H^{0}\left(\mathscr{O}_{\mathscr{L}\left(X, B_{X}\right)}\right) \rightarrow H^{1}\left(\mathscr{I}\left(X, B_{X}\right)\right)=0
$$

точна, если дивизор $-\left(K_{X}+B_{X}\right)$ численно эффективен и объемен, откуда следует, что

$$
\operatorname{LCS}\left(X, B_{X}\right)=\operatorname{Supp}\left(\mathscr{L}\left(X, B_{X}\right)\right)
$$

связно в случае, когда дивизор $-\left(K_{X}+B_{X}\right)$ численно эффективен и объемен.

Рассмотрим несколько элементарных применений теоремы 2.7 (ср. с примером 1.10).

Лемма 2.8. Предположим, что $\operatorname{LCS}\left(X, B_{X}\right) \neq \varnothing$, где $X \cong \mathbb{P}^{n} u B_{X} \sim_{\mathbb{Q}}$ $-\lambda K_{X}$ для некоторого рационального числа $0<\lambda<n /(n+1)$. Тогда выполнено неравенство $\operatorname{dim}\left(\operatorname{LCS}\left(X, B_{X}\right)\right) \geqslant 1$, а подсхема $\mathscr{L}\left(X, B_{X}\right)$ не содержит изолированных нульмерных компонент.

ДоказАтельство. Пусть $O \in X$ - точка такая, что $\operatorname{LCS}\left(X, \lambda B_{X}\right)=O \cup \Sigma$, где $\Sigma \subset X-$ (возможно, пустое) подмножество такое, что $O \notin \Sigma$.

Пусть $H$ - общая прямая в $X \cong \mathbb{P}^{2}$. Тогда множество $\operatorname{LCS}\left(X, \lambda B_{X}+H\right)=$ $O \cup H \cup \Sigma$ несвязно, но дивизор $-\left(K_{X}+\lambda B_{X}+H\right)$ является обильным, что противоречит теореме 2.7 .

Лемма 2.9. Предположим, что $\operatorname{LCS}\left(X, B_{X}\right) \neq \varnothing$, где $X \cong \mathbb{P}^{3}$ u $B_{X} \sim_{\mathbb{Q}}$ $-\lambda K_{X}$ для некоторого рационального числа $0<\lambda<1 / 2$. Тогда $\mathbb{L} \mathbb{C} S\left(X, B_{X}\right)$ содержит поверхность.

ДоказАтельство. Предположим, что $\mathbb{L} \mathbb{C} S\left(X, B_{X}\right)$ не содержит поверхностей. Пусть $S$ - общая плоскость в $\mathbb{P}^{3}$. Тогда множество $\operatorname{LCS}\left(\mathbb{P}^{3}, B_{X}+S\right)$ связно по теореме 2.7. Значит, лог-пара $\left(S,\left.B_{X}\right|_{S}\right)$ не лог-терминальна согласно замечанию 2.3. С другой стороны, множество $\operatorname{LCS}\left(S,\left.B_{X}\right|_{S}\right)$ состоит из конечного числа точек, что противоречит лемме 2.8 . 
Лемма 2.10. Предположим, что $\operatorname{LCS}\left(X, B_{X}\right) \neq \varnothing$, где $X-$ гладкая трехмерная квадрика в $\mathbb{P}^{4}$ и $B_{X} \sim_{\mathbb{Q}}-\lambda K_{X}$ для некоторого рачионального числа $0<\lambda<1 / 2$. Тогда $\mathbb{L} \mathbb{C S}\left(X, B_{X}\right)$ содержит поверхность.

ДокАЗАтельство. Пусть $L \subset X$ - общая прямая, пусть $P_{1} \in L \ni P_{2}-$ две общие точки, пусть $H_{1}$ и $H_{2}$ - гиперплоские сечения квадрики $X \subset \mathbb{P}^{4}$, касающиеся ее в точках $P_{1}$ и $P_{2}$ соответственно. Тогда множество

$$
\operatorname{LCS}\left(X, \lambda B_{X}+\frac{3}{4}\left(H_{1}+H_{2}\right)\right)=\operatorname{LCS}\left(X, \lambda B_{X}\right) \cup L
$$

несвязно, что противоречит утверждению теоремы 2.7 .

ЗАмЕчАнИЕ 2.11. Утверждения лемм 2.9, 2.10 (и 2.28) можно доказать другим способом. Предположим, что $B_{X} \sim_{\mathbb{Q}}-\lambda K_{X}$ для некоторого $\lambda \in \mathbb{Q}$ такого, что $0<\lambda<1 / 2$, где $X$ есть либо $\mathbb{P}^{3}$, либо $\mathbb{P}^{1} \times \mathbb{P}^{2}$, либо гладкая трехмерная квадрика. Допустим, что множество $\mathbb{L} \mathbb{C} S\left(X, B_{X}\right)$ не содержит поверхностей. Тогда $\operatorname{LCS}\left(X, B_{X}\right) \subseteq \Sigma$, где $\Sigma \subset X$ является (возможно, приводимой) кривой. Для общего автоморфизма $\varphi \in \operatorname{Aut}(X)$ имеем $\varphi(\Sigma) \cap \Sigma=\varnothing$, откуда следует, что $\operatorname{LCS}\left(X, \varphi\left(B_{X}\right)\right) \cap \operatorname{LCS}\left(X, B_{X}\right)=\varnothing$. Можно показать, что если $\varphi$ общий, то

$$
\operatorname{LCS}\left(X, \varphi\left(B_{X}\right)+B_{X}\right)=\operatorname{LCS}\left(X, \varphi\left(B_{X}\right)\right) \cup \operatorname{LCS}\left(X, B_{X}\right),
$$

что противоречит теореме 2.7 , поскольку $\lambda<1 / 2$.

Лемма 2.12. Предположим, что $\operatorname{LCS}\left(X, B_{X}\right) \neq \varnothing$, где $X$ является раздутием $\mathbb{P}^{3}$ в одной точке, и $B_{X} \sim_{\mathbb{Q}}-\lambda K_{X}$ для некоторого рационального числа $0<\lambda<1 / 2$. Тогда множество $\mathbb{L} \mathbb{C} S\left(X, B_{X}\right)$ содержит поверхность.

ДокАЗАтельство. Предположим, что множество $\mathbb{L} \mathbb{C} S\left(X, B_{X}\right)$ не содержит поверхностей. Пусть $\alpha: X \rightarrow \mathbb{P}^{3}$ - раздутие точки, и пусть $E$ - исключительный дивизор раздутия $\alpha$. В случае, когда $\operatorname{LCS}\left(X, \lambda B_{X}\right) \nsubseteq E$, мы можем применить лемму 2.9 к лог-паре $\left(\mathbb{P}^{3}, \alpha\left(B_{X}\right)\right)$ и сразу получить противоречие. Таким образом, можно считать, что $\operatorname{LCS}\left(X, B_{X}\right) \subseteq E$.

Пусть $H \subset \mathbb{P}^{3}$ - общая гиперплоскость, и пусть $H_{1} \subset \mathbb{P}^{3} \supset H_{2}$ - общие гиперплоскости, проходящие через точку $\alpha(E)$. Обозначим символами $\bar{H}, \bar{H}_{1}$ и $\bar{H}_{2}$ собственные прообразы гиперплоскостей $H, H_{1}$ и $H_{2}$ на многообразии $X$ соответственно. Тогда множество

$$
\operatorname{LCS}\left(X, B_{X}+\frac{1}{2}\left(\bar{H}_{1}+\bar{H}_{2}+2 \bar{H}\right)\right)
$$

несвязно, что противоречит теореме 2.7 .

Лемма 2.13. Пусть $X-$ конус в $\mathbb{P}^{4}$ над неособой двумерной квадрикой. Предположим, что $B_{X} \sim \mathbb{Q}-\lambda K_{X}$ для некоторого рационального числа $0<$ $\lambda<1 / 3$. Тогда $\mathbb{L} \mathbb{C} S\left(X, B_{X}\right)=\varnothing$.

ДоказАтельство. Предположим, что $\mathbb{L} \mathbb{C} S\left(X, B_{X}\right) \neq \varnothing$. Пусть $S$ - общее гиперплоское сечение конуса $X \subset \mathbb{P}^{4}$. Тогда $\operatorname{LCS}\left(S,\left.B_{X}\right|_{S}\right)=\varnothing$, поскольку $S \cong \mathbb{P}^{1} \times \mathbb{P}^{1}$ и $\operatorname{lct}\left(\mathbb{P}^{1} \times \mathbb{P}^{1}\right)=1 / 2$ (см. пример 1.10$)$. Таким образом, согласно замечанию $2.3,\left|\operatorname{LCS}\left(X, B_{X}\right)\right|<+\infty$. Тогда множество $\operatorname{LCS}\left(X, B_{X}+S\right)$ несвязно, что противоречит теореме 2.7 . 
Следующий результат является следствием теоремы 2.6 (см. [20; теорема 4.1]).

ЛЕмма 2.14. Предположим, что дивизор $-\left(K_{X}+B_{X}\right)$ численно эффективен и обгемен $и \operatorname{dim}\left(\operatorname{LCS}\left(X, B_{X}\right)\right)=1$. Тогда:

(а) множество $\operatorname{LCS}\left(X, B_{X}\right)$ является связным объединением гладких рациональных кривых,

(b) каждые две неприводимые компоненты множества $\operatorname{LCS}\left(X, B_{X}\right)$ пересекаются не более чем в одной точке,

(с) каждая пара пересекающихся неприводимых компонент множества $\operatorname{LCS}\left(X, B_{X}\right)$ пересекается трансверсально,

(d) никакие три неприводимые компоненты множества $\operatorname{LCS}\left(X, B_{X}\right)$ не пересекаются в одной точке,

(е) множество $\operatorname{LCS}\left(X, B_{X}\right)$ не содержит ииклов ("колес"), состоящих из неособых рациональных кривых.

ДокАЗАтЕльство. Рассуждая как в доказательстве теоремы 2.7, мы видим, что $\operatorname{LCS}\left(X, B_{X}\right)$ является связным деревом неособых рациональных кривых, которые пересекаются трансверсально.

Лемма 2.15 [43]. Пусть $X$ - гладкая гиперповерхность в $\mathbb{P}^{m}$ и выполнена эквивалентность $\left.B_{X} \sim_{\mathbb{Q}} \mathscr{O}_{\mathbb{P} m}(1)\right|_{X}$. Пусть $S \subsetneq X$ - неприводимое подмногообразие такое, ито $\operatorname{dim}(S) \geqslant k$. Тогда $\operatorname{mult}_{S}\left(B_{X}\right) \leqslant 1$.

Теперь рассмотрим одно простое применение теоремы 2.7 и леммы 2.15 .

Лемма 2.16. Пусть $X$ - кубическая гиперповерхность в $\mathbb{P}^{4}$, имеющая не более чем изолированные особенности. Предположим, что $B_{X} \sim_{\mathbb{Q}}-K_{X}$, но существует некоторое положительное рациональное число $\lambda<1 / 2$ такое, что $\operatorname{LCS}\left(X, \lambda B_{X}\right) \neq \varnothing$. Тогда $\operatorname{LCS}\left(X, \lambda B_{X}\right)=L$, где $L-$ прямая в $X \subset \mathbb{P}^{4}$ такая, что $L \cap \operatorname{Sing}(X) \neq \varnothing$.

ДоказатЕЛЬСтво. Пусть $S$ - общее гиперплоское сечение кубики $X$. Тогда

$$
S \cup \operatorname{LCS}\left(X, \lambda B_{X}\right) \subseteq \operatorname{LCS}\left(X, \lambda B_{X}+S\right),
$$

откуда по теореме 2.7 следует, что $\operatorname{dim}\left(\operatorname{LCS}\left(X, \lambda B_{X}\right)\right) \geqslant 1$. Поэтому множество $\operatorname{LCS}\left(S,\left.\lambda B_{X}\right|_{S}\right)$ непусто согласно замечанию 2.3. С другой стороны, по лемме 2.15 множество $\operatorname{LCS}\left(S,\left.\lambda B_{X}\right|_{S}\right)$ состоит из конечного числа точек. Таким образом, по теореме 2.7 существует единственная точка $O \in S$ такая, что $\operatorname{LCS}\left(S,\left.\lambda B_{X}\right|_{S}\right)=O$. Теперь из замечания 2.3 следует, что существует прямая $L \subset X$ такая, что $\operatorname{LCS}\left(X, \lambda B_{X}\right)=L$.

Рассуждая как в доказательстве леммы 2.15, мы видим, что $L \cap \operatorname{Sing}(X) \neq \varnothing$.

По аналогии с леммой 2.16 можно доказать следующий результат.

Лемма 2.17. Пусть дано двулистное накрытие $\tau: X \rightarrow \mathbb{P}^{3}$, которое разветвлено над неприводимой приведенной поверхностью $R \subset \mathbb{P}^{3}$ степени 4, имеющей не более чем обыкновенные двойные точки. Предположим, что выполнена эквивалентность $B_{X} \sim_{\mathbb{Q}}-\lambda K_{X}$, но $\operatorname{LCS}\left(X, B_{X}\right) \neq \varnothing$, где $\lambda<1 / 2$. Тогда $\operatorname{Sing}(X) \neq \varnothing u \operatorname{LCS}\left(X, B_{X}\right)=L$, где $L-$ неприводимая кривая на многообразии $X$ такая, что $-K_{X} \cdot L=2$ u $L \cap \operatorname{Sing}(X) \neq \varnothing$. 
ДокАЗАтельство. Заметим, что $-K_{X} \sim 2 H$, где $H-$ дивизор Картье на многообразии $X$ такой, что $H \sim \tau^{*}\left(\mathscr{O}_{\mathbb{P}^{3}}(1)\right)$. Многообразие $X$ является трехмерным многообразием Фано и $H^{3}=2$. В частности, по теореме 2.7 множество $\operatorname{LCS}\left(X, B_{X}+H\right)$ обязано быть связным. Значит, существует кривая $C \in \mathbb{L} \mathbb{C S}\left(X, B_{X}\right)$, откуда следует, что $\operatorname{mult}_{C}\left(B_{X}\right) \geqslant 1 / \lambda>2$.

Пусть $S$ - общая поверхность в $|H|$. Положим $B_{S}=\left.B_{X}\right|_{S}$. Тогда выполнена эквивалентность $-\lambda K_{S} \sim_{\mathbb{Q}} B_{S}$, но лог-пара $\left(S, B_{S}\right)$ не является лог-канонической в каждой точке пересечения $S \cap \operatorname{LCS}\left(X, B_{X}\right)$.

Поверхность $H$ является неособой гиперповерхностью в $\mathbb{P}(1,1,1,2)$ степени 4.

Пусть $P$ - любая точка в $S \cap \operatorname{LCS}\left(X, B_{X}\right)$. Тогда существует бирациональный морфизм $\rho: S \rightarrow \bar{S}$ такой, что $\bar{S}-$ кубическая поверхность в $\mathbb{P}^{3}$, а $\rho$ - изоморфизм в окрестности точки $P$. В частности, особенности лог-пары $\left(\bar{S}, \rho\left(B_{S}\right)\right)$ не являются лог-терминальными в точке $\rho(P)$. Таким образом, $\operatorname{LCS}\left(\bar{S}, \rho\left(B_{S}\right)\right) \neq \varnothing$, но

$$
\frac{1}{\lambda} \rho\left(B_{S}\right) \sim_{\mathbb{Q}}-\left.K_{\bar{S}} \sim \mathscr{O}_{\mathbb{P}^{3}}(1)\right|_{\bar{S}}
$$

откуда по лемме 2.15 и теореме 2.7 следует, что $\operatorname{LCS}\left(\bar{S}, \rho\left(B_{S}\right)\right)$ состоит из одной точки. Тогда

$$
P=S \cap C=S \cap \operatorname{LCS}\left(X, B_{X}\right),
$$

если точка $P$ выбрана достаточно общим образом. Значит, $\operatorname{LCS}\left(X, B_{X}\right)=C$, кривая $C$ неприводима и $-K_{X} \cdot C=2$. В частности, кривая $\tau(C) \subset \mathbb{P}^{3}$ является прямой.

Предположим, что $C \cap \operatorname{Sing}(X)=\varnothing$. Покажем, что это предположение приводит к противоречию.

Предположим, что $\tau(C) \subset R$. Рассмотрим общую точку $O \in C$. Рассмотрим плоскость $\tau(O) \in \Pi \subset \mathbb{P}^{3}$, которая касается поверхности $R$ в точке $\tau(O)$. Рассуждая как в доказательства леммы 2.15 (см. [43]), мы видим, что схема $\left.R\right|_{\Pi}$ приведена в общей точке кривой $\tau(C)$, поскольку $\tau(C) \cap \operatorname{Sing}(R)=\varnothing$. Зафиксируем общую прямую $\Gamma \subset \Pi \subset \mathbb{P}^{3}$ такую, что $\tau(O) \in \Gamma$. Пусть $\bar{\Gamma} \subset X-$ неприводимая кривая такая, что $\tau(\bar{\Gamma})=\Gamma$. Тогда $\bar{\Gamma} \not \subseteq \operatorname{Supp}\left(B_{X}\right)$, так как прямая $\Gamma$ заметает плотное подмножество в $\mathbb{P}^{3}$ при варьировании точки $O \in C$ и прямой $\Gamma \subset$ П. Отметим, что либо $H \cdot \bar{\Gamma}=1$, либо $H \cdot \bar{\Gamma}=2$. В последнем случае $\operatorname{mult}_{O}(\bar{\Gamma})=2$. Тогда

$$
H \cdot \bar{\Gamma}>2 \lambda H \cdot \bar{\Gamma}=\bar{\Gamma} \cdot B_{X} \geqslant \operatorname{mult}_{O}(\bar{\Gamma}) \operatorname{mult}_{C}\left(B_{V}\right) \geqslant H \cdot \bar{\Gamma},
$$

что является противоречием. Итак, показано, что $\tau(C) \not \subset R$.

Существует неприводимая приведенная кривая $\bar{C} \subset X$ такая, что $\tau(\bar{C})=$ $\tau(C) \subset \mathbb{P}^{3}$, но $\bar{C} \neq C$. Пусть $Y$ - общая поверхность в $|H|$, проходящая через кривые $\bar{C}$ и $C$. Тогда поверхность $Y$ неособа, поскольку $C \cap \operatorname{Sing}(X)=\varnothing$. Легко видеть, что $\bar{C} \cdot \bar{C}=C \cdot C=-2$ на поверхности $Y$. Очевидно, что $Y \not \subset \operatorname{Supp}\left(B_{X}\right)$. Положим $B_{Y}=\left.B_{X}\right|_{Y}$. Тогда

$$
B_{Y}=\operatorname{mult}_{\bar{C}}\left(B_{X}\right) \bar{C}+\operatorname{mult}_{C}\left(B_{X}\right) C+\Delta,
$$

где $\Delta$ - эффективный $\mathbb{Q}$-дивизор на поверхности $Y$ такой, что

$$
\bar{C} \not \subset \operatorname{Supp}(\Delta) \not \supset C .
$$


С другой стороны, $B_{Y} \sim_{\mathbb{Q}} 2 \lambda(\bar{C}+C)$, откуда следует, в частности, что

$$
\begin{aligned}
\left(2 \lambda-\operatorname{mult}_{C}\left(B_{X}\right)\right) C \cdot C & =\left(\operatorname{mult}_{\bar{C}}\left(B_{X}\right)-2 \lambda\right) \bar{C} \cdot C+\Delta \cdot C \\
& \geqslant\left(\operatorname{mult}_{\bar{C}}\left(B_{X}\right)-2 \lambda\right) \bar{C} \cdot C \geqslant 0,
\end{aligned}
$$

так как $\Delta \cdot C \geqslant 0$ и $\bar{C} \cdot C \geqslant 0$. Тогда $\operatorname{mult}_{\bar{C}}\left(B_{X}\right) \geqslant 2 \lambda$, поскольку $C \cdot C<0$. Отсюда

$$
-\Delta \sim_{\mathbb{Q}}\left(\operatorname{mult}_{\bar{C}}\left(B_{X}\right)-2 \lambda\right) \bar{C}+\left(\operatorname{mult}_{C}\left(B_{X}\right)-2 \lambda\right) C,
$$

что является противоречием, так как $\operatorname{mult}_{C}\left(B_{X}\right)>2 \lambda$, а поверхность $Y$ проективна.

Утверждение теоремы 2.7 можно обобщить следующим образом (см. [40; лемма 5.7]).

Tеорема 2.18. Пусть $\psi: X \rightarrow Z$ - морфизм. Тогда $\operatorname{LCS}\left(\bar{X}, B^{\bar{X}}\right)$ связно в окрестности каждого слоя морфизма $\psi \circ \pi: X \rightarrow Z$, если выполнены следующие условия:

(а) морфизм $\psi$ сюргективен и имеет связные слои,

(b) дивизор $-\left(K_{X}+B_{X}\right)$ численно эфбективен и обгемен относительно $\psi$.

Рассмотрим одно важное применение теоремы 2.18 (см. [41; теорема 5.50]).

Теорема 2.19. Пусть $B_{1}$ - дивизор Картъе, $a_{1}=1$, а многообразие $B_{1}$ имеет лог-терминальные особенности. Тогда следующие утверждения эквивалентны:

(а) лог-пара $\left(X, B_{X}\right)$ имеет лог-канонические особенности в окрестности дивизора $B_{1}$

(b) особенности лог-парь $\left(B_{1},\left.\sum_{i=2}^{r} a_{i} B_{i}\right|_{B_{1}}\right)$ лог-канонические.

Простое применение теоремы 2.19 дает интересный результат (см. [41; следствие 5.57]).

Лемма 2.20. Предположим, что $\operatorname{dim}(X)=2 u a_{1} \leqslant 1$. Тогда выполнено неравенство $\left(\sum_{i=2}^{r} a_{i} B_{i}\right) B_{1}>1$, если лог-пара $\left(X, B_{X}\right)$ не лог-канонична в точке $O \in B_{1}$ такой, что $O \notin \operatorname{Sing}(X) \cup \operatorname{Sing}\left(B_{1}\right)$.

ДокАЗАТЕЛЬСтво. Предположим, что $\left(X, B_{X}\right)$ не является лог-канонической в точке $O \in B_{1}$. По теореме 2.19 имеем

$$
\left(\sum_{i=2}^{r} a_{i} B_{i}\right) \cdot B_{1} \geqslant \operatorname{mult}_{O}\left(\left.\sum_{i=2}^{r} a_{i} B_{i}\right|_{B_{1}}\right)>1,
$$

если $O \notin \operatorname{Sing}(X) \cup \operatorname{Sing}\left(B_{1}\right)$, поскольку $\left(X, B_{1}+\sum_{i=2}^{r} a_{i} B_{i}\right)$ не лог-канонична в точке $O$.

Рассмотрим еще одно применение теоремы 2.19 (ср. с леммой 2.29).

Лемма 2.21. Пусть $X$ - многообразие Фано, имеющее не более чем логтерминальные особенности. Тогда $\operatorname{lct}\left(\mathbb{P}^{1} \times X\right)=\min (1 / 2, \operatorname{lct}(X))$. 
ДокАзАтельство. Неравенства $1 / 2 \geqslant \operatorname{lct}(V \times U) \leqslant \operatorname{lct}(X)$ очевидны. Предположим, что выполнено неравенство $\operatorname{lct}\left(\mathbb{P}^{1} \times X\right)<\min (1 / 2, \operatorname{lct}(X))$. Покажем, что сделанное предположение приводит к противоречию.

Существует эффективный $\mathbb{Q}$-дивизор $D \sim_{\mathbb{Q}}-K_{\mathbb{P}^{1} \times X}$ такой, что лог-пара $\left(\mathbb{P}^{1} \times X, \lambda D\right)$ не является лог-канонической в некоторой точке $P \in \mathbb{P}^{1} \times X$, где $\lambda<\min (1 / 2, \operatorname{lct}(X))$.

Пусть $F$ - слой естественной проекции $\mathbb{P}^{1} \times X \rightarrow \mathbb{P}^{1}$ такой, что $P \in F$. Тогда $D=\mu F+\Omega$, где $\Omega$ - эффективный $\mathbb{Q}$-дивизор на $\mathbb{P}^{1} \times X$ такой, что $F \not \subset \operatorname{Supp}(\Omega)$.

Пусть $L$ - общий слой естественной проекции $\mathbb{P}^{1} \times X \rightarrow X$. Тогда

$$
2=D \cdot L=\mu+\Omega \cdot L \geqslant \mu,
$$

откуда следует, что лог-пара $\left(\mathbb{P}^{1} \times X, F+\lambda \Omega\right)$ также не лог-канонична в точке $P$. Тогда лог-пара $\left(F,\left.\lambda \Omega\right|_{F}\right)$ не лог-канонична в точке $P$ по теореме 2.19 , но $\left.\Omega\right|_{F} \sim_{\mathbb{Q}}$ $-K_{F}$, что является противоречием, поскольку $X \cong F$ и $\lambda<\operatorname{lct}(X)$.

Пусть $P$ - точка многообразия $X$. Рассмотрим некоторый эффективный дивизор

$$
\Delta=\sum_{i=1}^{r} \varepsilon_{i} B_{i} \sim_{\mathbb{Q}} B_{X},
$$

где $\varepsilon_{i}-$ неотрицательное рациональное число. Предположим, что дивизор $\Delta$ является $\mathbb{Q}$-Картье дивизором, выполнена эквивалентность $\Delta \sim_{\mathbb{Q}} B_{X}$ и особенности лог-пары $(X, \Delta)$ являются лог-каноническими в точке $P \in X$.

ЗАмечАниЕ 2.22. Предположим, что лог-пара $\left(X, B_{X}\right)$ не лог-канонична в точке $P \in X$. Положим $\alpha=\min \left\{a_{i} / \varepsilon_{i} \mid \varepsilon_{i} \neq 0\right\}$, где число $\alpha$ корректно определено, поскольку не все числа $\varepsilon_{1}, \ldots, \varepsilon_{r}$ обращаются в нуль. Тогда $\alpha<1$, а особенности лог-пары

$$
\left(X, \sum_{i=1}^{r} \frac{a_{i}-\alpha \varepsilon_{i}}{1-\alpha} B_{i}\right)
$$

не являются лог-каноническими в точке $P \in X$. При этом

$$
\sum_{i=1}^{r} \frac{a_{i}-\alpha \varepsilon_{i}}{1-\alpha} B_{i} \sim_{\mathbb{Q}} B_{X} \sim_{\mathbb{Q}} \Delta,
$$

но по крайней мере одна неприводимая компонента дивизора $\operatorname{Supp}(\Delta)$ не содержится в

$$
\operatorname{Supp}\left(\sum_{i=1}^{r} \frac{a_{i}-\alpha \varepsilon_{i}}{1-\alpha} B_{i}\right) .
$$

Утверждение замечания 2.22 очевидно, но тем не менее оно очень полезно.

Лемма 2.23. Предположим, что $X \cong C_{1} \times C_{2}$, где $C_{1}$ и $C_{2}$ - гладкие кривые. Предположим, что $B_{X} \sim_{\mathbb{Q}} \lambda E+\mu F$, где $E \cong C_{1} u F \cong C_{2}-$ кривые на поверхности $X$ такие, что $E \cdot E=F \cdot F=0 u E \cdot F=1$, a $\lambda u \mu$ неотрицательные рациональные числа. Тогда

(а) лог-пара $\left(X, B_{X}\right)$ имеет лог-терминальные особенности, если выполнены неравенства $\lambda<1$ и $\mu<1$, 
(b) лог-пара $\left(X, B_{X}\right)$ имеет лог-канонические особенности, если выполнены неравенства $\lambda \leqslant 1$ u $\mu \leqslant 1$.

ДокАзАтельство. Достаточно доказать утверждение (а). Предположим, что $\lambda, \mu<1$, но особенности лог-пары $\left(X, B_{X}\right)$ не являются лог-терминальными в некоторой точке $P \in X$. Тогда $\operatorname{mult}_{P}\left(B_{X}\right) \geqslant 1$ и, согласно замечанию 2.22 , мы можем считать, что либо $E \not \subset \operatorname{Supp}\left(B_{X}\right)$, либо $F \not \subset \operatorname{Supp}\left(B_{X}\right)$. С другой стороны, $E \cdot B_{X}=\mu$ и $F \cdot B_{X}=\lambda$, откуда сразу следует противоречие c $\operatorname{mult}_{P}\left(B_{X}\right) \geqslant 1$.

Пусть $\left[B_{X}\right]-$ класс $\mathbb{Q}$-рациональной эквивалентности дивизора $B_{X}$. Положим

$$
\begin{gathered}
\operatorname{lct}\left(X,\left[B_{X}\right]\right)=\inf \{\operatorname{lct}(X, D) \mid D-\text { эффективный } \mathbb{Q} \text {-дивизор } \\
\text { такой, что } \left.D \sim_{\mathbb{Q}} B_{X}\right\} \geqslant 0
\end{gathered}
$$

и будем считать, что $\operatorname{lct}\left(X,\left[B_{X}\right]\right)=+\infty$, если $B_{X}=0$. Отметим, что $B_{X}-$ эффективный дивизор.

ЗАмЕчАНИЕ 2.24. Выполнено равенство $\operatorname{lct}\left(X,\left[-K_{X}\right]\right)=\operatorname{lct}(X)$ (см. определение 1.2).

Рассуждая как в доказательстве леммы 2.21, можно получить следующий результат.

ЛЕмма 2.25. Пусть $\varphi: X \rightarrow Z$ - сюрвективный морфизм со связными слоями такой, что $\operatorname{dim} Z=1$. Пусть $F$ - слой морфизма $\varphi$, который имеет не более чем лог-терминальные особенности. Тогда либо $\operatorname{lct}_{F}\left(X, B_{X}\right) \geqslant$ $\operatorname{lct}\left(F,\left[\left.B_{X}\right|_{F}\right]\right)$, либо существует рачиональное число $0<\varepsilon<\operatorname{lct}\left(F,\left[\left.B_{X}\right|_{F}\right]\right)$ maкое, что $F \subseteq \operatorname{LCS}\left(X, \varepsilon B_{X}\right)$.

ДоказАтельство. Предположим, что $\operatorname{lct}_{F}\left(X, B_{X}\right)<\operatorname{lct}\left(F,\left[\left.B_{X}\right|_{F}\right]\right)$. Тогда существует рациональное число $\varepsilon<\operatorname{lct}\left(F,\left[\left.B_{X}\right|_{F}\right]\right)$ такое, что особенности лог-пары $\left(X, \varepsilon B_{X}\right)$ не являются лог-каноническими в некоторой точке $P \in F$. Положим $B_{X}=\mu F+\Omega$, где $\Omega$ - эффективный $\mathbb{Q}$-дивизор на многообразии $X$ такой, что $F \not \subset \operatorname{Supp}(\Omega)$.

Можно считать, что $\varepsilon \mu \leqslant 1$. Тогда лог-пара $(X, F+\varepsilon \Omega)$ не лог-канонична в точке $P$. Особенности лог-пары $\left(F,\left.\varepsilon \Omega\right|_{F}\right)$ также не являются лог-каноническими в точке $P$ по теореме 2.19, но $\left.\left.\Omega\right|_{F} \sim_{\mathbb{Q}} B_{X}\right|_{F}$, что является противоречием.

Приведем одно несложное применение леммы 2.25 .

Лемма 2.26. Пусть $Q \subset \mathbb{P}^{4}$ - конус над гладкой квадратичной поверхностъю, и пусть $\alpha: X \rightarrow Q-$ раздутие неособой коники $C \subset Q \backslash \operatorname{Sing}(Q)$. Тогда $\operatorname{lct}(X)=1 / 3$.

ДокАЗАТЕльство. Пусть $H$ - общее гиперплоское сечение конуса $Q \subset \mathbb{P}^{4}$, которое содержит кривую $C$, и пусть $\bar{H}$ - собственный прообраз поверхности $H$ на многообразии $X$. Тогда $-K_{X} \sim 3 \bar{H}+2 E$, где $E-$ исключительный дивизор раздутия $\alpha$. В частности, имеет место неравенство $\operatorname{lct}(X) \leqslant 1 / 3$.

Предположим, что $\operatorname{lct}(X)<1 / 3$. Тогда существует эффективный $\mathbb{Q}$-дивизор $D \sim_{\mathbb{Q}}-K_{X}$ такой, что особенности лог-пары $(X, \lambda D)$ не являются лог-канони- 
ческими для некоторого положительного рационального числа $\lambda<1 / 3$. Существует коммутативная диаграмма

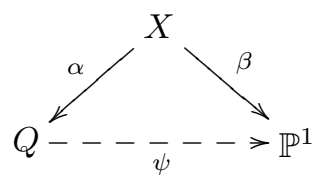

где $\beta$ - морфизм, заданный линейной системой $|\bar{H}|$, а $\psi$ - проекция из двумерного линейного подпространства, которое содержит конику $C$.

Предположим, что $\mathbb{L} \mathbb{C} S(X, \lambda D)$ содержит поверхность $M \subset X$. Тогда $D=$ $\mu M+\Omega$, где $\mu \geqslant 1 / \lambda$, а $\Omega$ - эффективный $\mathbb{Q}$-дивизор такой, что $M \not \subset \operatorname{Supp}(\Omega)$.

Пусть $F$ - общий слой морфизма $\beta$. Тогда $F \cong \mathbb{P}^{1} \times \mathbb{P}^{1}$ и $\left.D\right|_{F} \sim_{\mathbb{Q}}-K_{F}$, откуда сразу следует, что $M-$ слой морфизма $\beta$, но $\alpha(D) \sim_{\mathbb{Q}}-K_{Q} \sim 3 \alpha(M)$, что является противоречием, поскольку $\mu \geqslant 1 / \lambda>3$. Значит, множество $\mathbb{L} \mathbb{C} \mathbb{S}(X, \lambda D)$ не содержит поверхностей.

Существует слой $S$ морфизма $\beta$ такой, что $S \neq S \cap \operatorname{LCS}(X, \lambda D) \neq \varnothing$, откуда по лемме 2.25 следует, что $S$ особа, поскольку $\operatorname{lct}\left(\mathbb{P}^{1} \times \mathbb{P}^{1}\right)=1 / 2$.

Значит, поверхность $S$ является неприводимым квадратичным конусом в $\mathbb{P}^{3}$. Тогда $\operatorname{LCS}(X, \lambda D) \subseteq S$ по теореме 2.7. Более того, поскольку лог-пара $(X$, $\left.S+\frac{2}{3} E\right)$ имеет лог-канонические особенности и выполнена эквивалентность $3 S+2 E \sim_{\mathbb{Q}} D$, мы можем считать, согласно замечанию 2.22 , что либо $S \not \subset$ $\operatorname{Supp}(D)$, либо $E \not \subset \operatorname{Supp}(D)$.

Положим $\Gamma=E \cap S$. Кривая $\Gamma$ является неприводимой коникой. Тогда $\operatorname{LCS}(X, \lambda D) \subseteq \Gamma$ по лемме 2.13. Пересекая $D$ с общей образующей конуса $S \subset \mathbb{P}^{3}$ и с общим слоем проекции $E \rightarrow C$, мы видим, что $\Gamma \nsubseteq \operatorname{LCS}(X, \lambda D)$, откуда по теореме 2.7 сразу следует, что множество $\operatorname{LCS}(X, \lambda D)$ состоит ровно из одной точки $O \in \Gamma$.

Пусть $R$ - общая поверхность в линейной системе $\left|\alpha^{*}(H)\right|$. Тогда

$$
\operatorname{LCS}\left(X, \lambda D+\frac{1}{2}(\bar{H}+2 R)\right)=R \cup O,
$$

что противоречит теореме 2.7 , поскольку $-K_{X} \sim \bar{H}+2 R \sim_{\mathbb{Q}} D$ и $\lambda<1 / 3$.

Следующее обобщение леммы 2.25 вытекает из [54; предложение 5.19] (cp. [6]).

Теорема 2.27. Пусть $\varphi: X \rightarrow Z$ - сюрбективный плоский морфизм со связными слоями такой, что $Z$ имеет рациональные особенности, а схемные слои морфизма ч имеют не более чем канонические горенштейновы особенности. Пусть $F-$ схемный слой морфизма $\varphi$. Тогда либо $\operatorname{lct}_{F}\left(X, B_{X}\right) \geqslant$ $\operatorname{lct}\left(F,\left[\left.B_{X}\right|_{F}\right]\right)$, либо существует положительное рачиональное число $\varepsilon<$ $\operatorname{lct}\left(F,\left[\left.B_{X}\right|_{F}\right]\right)$ maкое, чmo $F \subseteq \operatorname{LCS}\left(X, \varepsilon B_{X}\right)$.

Приведем одно элементарное применение теоремы 2.27 .

Лемма 2.28. Предположим, что $\operatorname{LCS}\left(X, B_{X}\right) \neq \varnothing$, где $X \cong \mathbb{P}^{1} \times \mathbb{P}^{2}$ и въьполнена эквивалентность $B_{X} \sim_{\mathbb{Q}}-\lambda K_{X}$ для некоторого $0<\lambda<1 / 2$. Тогда множество $\mathbb{L} \mathbb{C} S\left(X, B_{X}\right)$ содержит поверхность. 
ДокаЗАТЕЛЬСтво. Предположим, что множество $\mathbb{L} \mathbb{C S}\left(X, B_{X}\right)$ не содержит поверхностей. Из теорем 2.7 и 2.27 следует, что $\operatorname{LCS}\left(X, B_{X}\right)=F$, где $F$ - некоторый слой естественной проекции $\pi_{2}: X \rightarrow \mathbb{P}^{2}$. Пусть $S$ - общая поверхность в линейной системе $\left|\pi_{1}^{*}\left(\mathscr{O}_{\mathbb{P}^{2}}(1)\right)\right|$, и пусть $M_{1}$ и $M_{2}$ - общие слои естественной проекции $\pi_{1}: X \rightarrow \mathbb{P}^{1}$. Тогда множество

$$
\operatorname{LCS}\left(X, \lambda D+\frac{1}{2}\left(M_{1}+M_{2}+3 S\right)\right)=F \cup S
$$

несвязно, что по теореме 2.7 является противоречием.

Лемма 2.29. Пусть $V$ и $U$-многообразия Фано, имеющие не более чем канонические горенштейновы особенности. Тогда $\operatorname{lct}(V \times U)=\min (\operatorname{lct}(V)$, $\operatorname{lct}(U))$.

ДоказатеЛЬСтво. Неравенства $\operatorname{lct}(V) \geqslant \operatorname{lct}(V \times U) \leqslant \operatorname{lct}(U)$ очевидны. Предположим, что выполнено неравенство $\operatorname{lct}(V \times U)<\min (\operatorname{lct}(V), \operatorname{lct}(U))$, и покажем, что сделанное предположение приводит к противоречию.

Существует эффективный $\mathbb{Q}$-дивизор $D \sim_{\mathbb{Q}}-K_{V \times U}$ такой, что лог-пара $(V \times U, \lambda D)$ не является лог-канонической в некоторой точке $P \in V \times U$, где $\lambda<\min (\operatorname{lct}(V), \operatorname{lct}(U))$.

Отождествим $V$ со слоем проекции $V \times U \rightarrow U$, который содержит $P$. Неравенства

$$
\operatorname{lct}(V)>\lambda>\operatorname{lct}_{V}(V \times U, D) \geqslant \operatorname{lct}\left(V,\left[\left.D\right|_{V}\right]\right)=\operatorname{lct}\left(V,\left[-K_{V}\right]\right)=\operatorname{lct}(V)
$$

несовместны. Поэтому из теоремы 2.27 следует, что особенности лог-пары $(V \times U, \lambda D)$ не являются лог-каноническими в каждой точке слоя $V \subset V \times U$.

Отождествим $U$ с общим слоем проекции $V \times U \rightarrow V$. Тогда $\left.D\right|_{U} \sim_{\mathbb{Q}}-K_{U}$, а лог-пара $\left(U,\left.\lambda D\right|_{U}\right)$ не является лог-канонической в точке $U \cap V$ по замечанию 2.3 (примененному $\operatorname{dim} V$ раз). Полученное утверждение противоречит неравенству $\lambda<\operatorname{lct}(U)$.

По-видимому, утверждение леммы 2.29 остается верным и для многообразий Фано с лог-терминальными особенностями (ср. с леммой 2.21).

\section{3. Кубические поверхности}

Пусть $X$ - кубическая поверхность в $\mathbb{P}^{3}$, имеющая не более чем одну особую точку, которая является обыкновенной двойной точкой.

ОПРеДЕЛЕНИЕ 3.1. Точка $O \in X$ называется точкой Эккарда, если $O \notin$ $\operatorname{Sing}(X)$ и $O=L_{1} \cap L_{2} \cap L_{3}$, где $L_{1}, L_{2}, L_{3}$ - различные прямые на поверхности $X \subset \mathbb{P}^{3}$.

На общей кубической поверхности нет точек Эккарда. Из примеров 1.10 и 1.11 следует, что

$$
\operatorname{lct}(X)= \begin{cases}3 / 4, & \text { если } X \text { не обладает точкой Эккарда и } \operatorname{Sing}(X)=\varnothing, \\ 2 / 3, & \text { если } X \text { обладает точкой Эккарда или } \operatorname{Sing}(X) \neq \varnothing .\end{cases}
$$


Пусть $D$ - эффективный $\mathbb{Q}$-дивизор на $X$ такой, что $D \sim_{\mathbb{Q}}-K_{X}$, и пусть $\omega \in \mathbb{Q}>0$ - число такое, что $\omega<3 / 4$. В данном разделе мы докажем следующий результат (ср. [5], [14]).

Теорема 3.2. Предположим, что особенности лог-пары $(X, \omega D)$ не лог-канонические. Тогда $\operatorname{LCS}(X, \omega D)=O$, где $O \in X$ является либо особой точкой, либо точкой Эккарда.

Предположим, что лог-пара $(X, \omega D)$ не лог-канонична. Пусть $P$ - произвольная точка множества $\operatorname{LCS}(X, \omega D)$. Предположим, что $P$ не является для поверхности $X$ ни особой точкой, ни точкой Эккарда.

Лемма 3.3. $\operatorname{LCS}(X, \omega D)=P$.

ДокАЗАТЕЛЬСтво. Предположим, что $\operatorname{LCS}(X, \omega D) \neq P$. Тогда, согласно теореме 2.7, существует кривая $C \subset X$ такая, что $P \in C \subseteq \operatorname{LCS}(X, \omega D)$. Значит, существует эффективный $\mathbb{Q}$-дивизор $\Omega$ на поверхности $X$ такой, что $C \not \subset \operatorname{Supp}(\Omega)$ и $D=\mu C+\Omega$, где $\mu \geqslant 1 / \omega$. Пусть $H$ - общее гиперплоское сечение поверхности $X$. Тогда

$$
3=H \cdot D=\mu H \cdot C+H \cdot \Omega \geqslant \mu \operatorname{deg} C,
$$

откуда следует, что либо $\operatorname{deg} C=1$, либо $\operatorname{deg} C=2$.

Пусть $\operatorname{deg} C=1$. Пусть $Z$ - общая коника на $X$ такая, что $-K_{X} \sim C+Z$. Тогда

$$
2=Z \cdot D=\mu Z \cdot C+Z \cdot \Omega \geqslant \mu Z \cdot C= \begin{cases}2 \mu, & \text { если } C \cap \operatorname{Sing}(X)=\varnothing, \\ 3 \mu / 2, & \text { если } C \cap \operatorname{Sing}(X) \neq \varnothing,\end{cases}
$$

откуда следует, что $\mu \leqslant 4 / 3$, что является противоречием, поскольку $\mu \geqslant$ $1 / \omega>4 / 3$.

Показано, что $\operatorname{deg} C=2$. Пусть $L-$ прямая на $X$ такая, что $-K_{X} \sim C+L$. Тогда $D=\mu C+\lambda L+\Upsilon$, где $\Upsilon$ - эффективный $\mathbb{Q}$-дивизор такой, что $C \not \subset$ $\operatorname{Supp}(\Upsilon) \not \supset L$. Имеем

$$
\begin{aligned}
1 & =L \cdot D=\mu L \cdot C+\lambda L \cdot L+L \cdot \Upsilon \geqslant \mu L \cdot C+\lambda L \cdot L \\
& = \begin{cases}2 \mu-\lambda, & \text { если } C \cap \operatorname{Sing}(X)=\varnothing, \\
3 \mu / 2-\lambda / 2, & \text { если } C \cap \operatorname{Sing}(X) \neq \varnothing,\end{cases}
\end{aligned}
$$

откуда следует, что $\mu \leqslant 7 / 6<4 / 3$, так как $\lambda \leqslant 4 / 3($ см. случай $\operatorname{deg} C=1)$. Таким образом, получено противоречие, поскольку выполнено неравенство $\mu>4 / 3$.

Пусть $\pi: U \rightarrow X$ - раздутие точки $P$, и пусть $E$ - исключительная кривая раздутия $\pi$. Тогда $\bar{D} \sim_{\mathbb{Q}} \pi^{*}(D)+\operatorname{mult}_{P}(D) E$, где $\operatorname{mult}_{P}(D) \geqslant 1 / \omega$, a $\bar{D}-$ собственный прообраз дивизора $D$ на поверхности $U$. Особенности лог-пары $\left(U, \omega \bar{D}+\left(\omega \operatorname{mult}_{P}(D)-1\right) E\right)$ не являются лог-каноническими в некоторой точке $Q \in E$. Тогда либо $\operatorname{mult}_{P}(D) \geqslant 2 / \omega$, либо

$$
\operatorname{mult}_{Q}(\bar{D})+\operatorname{mult}_{P}(D) \geqslant 2 / \omega>8 / 3
$$

поскольку дивизор $\omega \bar{D}+\left(\omega \operatorname{mult}_{P}(D)-1\right) E$ эффективен. 
Пусть $T$ - единственное гиперплоское сечение поверхности $X$, которое имеет особенность в точке $P$. Согласно замечанию 2.22 , можно считать, что $\operatorname{Supp}(T) \nsubseteq \operatorname{Supp}(D)$, поскольку лог-пара $(X, \omega T)$ лог-канонична. Кривая $T$ приведена. Таким образом, возможны следующие случаи: кривая $T$ является неприводимой и приведенной кубической кривой; кривая $T$ является объединением прямой и неприводимой коники; кривая $T$ является объединением трех различных прямых. Отметим, что $\operatorname{mult}_{P}(T)=2$, поскольку $P$ не является точкой Эккарда. В оставшейся части этого раздела мы исключим все эти три случая по отдельности.

Лемма 3.4. Кривая Т приводима.

ДокАЗАТЕльСтво. Предположим, что кривая $T$ является неприводимой кубической кривой. В этом случае существует коммутативная диаграмма

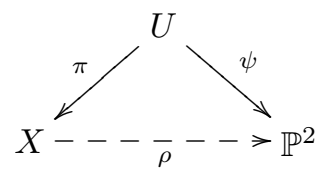

где $\psi$ - двулистное накрытие, разветвленное над кривой степени 4 , а $\rho$ - проекция из $P$.

Пусть $\bar{T}$ - собственный прообраз кривой $T$ на $U$. Предположим, что $Q \in \bar{T}$. Тогда

$$
\begin{aligned}
3-2 \operatorname{mult}_{P}(D) & =\bar{T} \cdot \bar{D} \geqslant \operatorname{mult}_{Q}(\bar{T}) \operatorname{mult}_{Q}(\bar{D}) \\
& >\operatorname{mult}_{Q}(\bar{T})\left(8 / 3-\operatorname{mult}_{P}(D)\right) \geqslant 8 / 3-\operatorname{mult}_{P}(D),
\end{aligned}
$$

откуда $\operatorname{mult}_{P}(D) \leqslant 1 / 3$. Последнее неравенство абсурдно. Значит, мы показали, что $Q \notin \bar{T}$.

Пусть $\tau \in \operatorname{Aut}(U)$ - естественная инволюция ${ }^{3}$, индуцированная двулистным накрытием $\psi$. В работе [42] показано, что

$$
\tau^{*}\left(\pi^{*}\left(-K_{X}\right)\right) \sim \pi^{*}\left(-2 K_{X}\right)-3 E
$$

и $\tau(\bar{T})=E$. Положим $\breve{Q}=\pi \circ \tau(Q)$. Тогда $\breve{Q} \neq P$, поскольку $Q \notin \bar{T}$.

Пусть $H$ - гиперплоское сечение поверхности $X$, которое имеет особенность в точке $\breve{Q}$. Тогда $T \neq H$, поскольку $P \neq \breve{Q}$, а кривая $T$ неособа вне точки $P$. Следовательно, $P \notin H$, так как в противном случае

$$
3=H \cdot T \geqslant \operatorname{mult}_{P}(H) \operatorname{mult}_{P}(T)+\operatorname{mult}_{\breve{Q}}(H) \operatorname{mult}_{\breve{Q}}(T) \geqslant 4 .
$$

Пусть $\bar{H}$ - собственный прообраз кривой $H$ на поверхности $U$. Положим $\bar{R}=\tau(\bar{H})$ и $R=\pi(\bar{R})$. Тогда $\bar{R} \sim \pi^{*}\left(-2 K_{X}\right)-3 E$, а кривая $\bar{R}$ обязана быть особой в точке $Q$.

Предположим, что кривая $R$ неприводима. Принимая во внимания все возможные особенности кривой $\bar{R}$, мы видим, что особенности лог-пары $\left(X, \frac{3}{8} R\right)$ являются лог-каноническими. Значит, согласно замечанию 2.22 , мы можем считать, что $R \nsubseteq \operatorname{Supp}(D)$. Тогда

$$
6-3 \operatorname{mult}_{P}(D)=\bar{R} \cdot \bar{D} \geqslant \operatorname{mult}_{Q}(\bar{R}) \operatorname{mult}_{Q}(\bar{D})>2\left(8 / 3-\operatorname{mult}_{P}(D)\right),
$$

\footnotetext{
${ }^{3}$ Инволюция $\tau$ индуцирует инволюцию в $\operatorname{Bir}(X)$, которую называют инволюцией Гейзера.
} 
откуда следует, что mult $P(D)<2 / 3$. Последнее неравенство абсурдно, посколь-

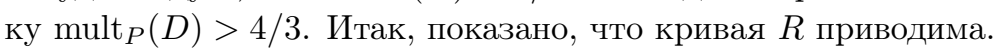

Кривые $R$ и $H$ приводимы. Значит, существует прямая $L \subset X$ такая, что $P \notin L \ni \breve{Q}$.

Пусть $\bar{L}$ - собственный прообраз прямой $L$ на $U$. Положим $\bar{Z}=\tau(\bar{L})$. Тогда $\bar{L} \cdot E=0$ и $\bar{L} \cdot \bar{T}=\bar{L} \cdot \pi^{*}\left(-K_{X}\right)=1$, откуда $\bar{Z} \cdot E=1$ и $\bar{Z} \cdot \pi^{*}\left(-K_{X}\right)=2$. Имеем $Q \in \bar{Z}$. Тогда

$$
2-\operatorname{mult}_{P}(D)=\bar{Z} \cdot \bar{D} \geqslant \operatorname{mult}_{Q}(\bar{D})>8 / 3-\operatorname{mult}_{P}(D)>2-\operatorname{mult}_{P}(D)
$$

в случае, когда $\bar{Z} \nsubseteq \operatorname{Supp}(\bar{D})$. Таким образом, мы видим, что $\bar{Z} \subseteq \operatorname{Supp}(\bar{D})$.

Положим $Z=\pi(\bar{Z})$. Тогда $Z$ является неприводимой коникой такой, что $P \in Z$ и $-K_{X} \sim L+Z$, откуда следует, что объединение $L \cup Z$ высекается плоскостью $\mathbb{P}^{3}$, которая содержит $Z$. Положим $D=\varepsilon Z+\Upsilon$, где $\Upsilon$ - эффективный $\mathbb{Q}$-дивизор такой, что $Z \not \subset \operatorname{Supp}(\Upsilon)$.

Можно считать, что $L \nsubseteq \operatorname{Supp}(\Upsilon)$ (см. замечание 2.22). Тогда

$$
1=L \cdot D=\varepsilon Z \cdot L+L \cdot \Upsilon \geqslant \varepsilon Z \cdot L= \begin{cases}2 \varepsilon, & \text { если } Z \cap \operatorname{Sing}(X)=\varnothing, \\ 3 \varepsilon / 2, & \text { если } Z \cap \operatorname{Sing}(X) \neq \varnothing,\end{cases}
$$

откуда следует, что $\varepsilon \leqslant 2 / 3$.

Пусть $\bar{\Upsilon}$ - собственный прообраз дивизора $\Upsilon$ на поверхности $U$. Тогда особенности лог-пары $\left(U, \varepsilon \omega \bar{Z}+\omega \bar{\Upsilon}+\left(\omega \operatorname{mult}_{P}(D)-1\right) E\right)$ не лог-каноничны в точке $Q \in \bar{Z}$. Следовательно,

$$
\omega \bar{\Upsilon} \cdot \bar{Z}+\left(\omega \operatorname{mult}_{P}(D)-1\right)=\left(\omega \bar{\Upsilon}+\left(\omega \operatorname{mult}_{P}(D)-1\right) E\right) \cdot \bar{Z}>1
$$

по лемме 2.20 , поскольку $\varepsilon \leqslant 2 / 3$. В частности, мы видим, что

$$
\begin{aligned}
8 / 3-\operatorname{mult}_{P}(D)<\bar{Z} \cdot \bar{\Upsilon} & =2-\operatorname{mult}_{P}(D)-\varepsilon \bar{Z} \cdot \bar{Z} \\
& = \begin{cases}2-\operatorname{mult}_{P}(D)+\varepsilon, & \text { если } Z \cap \operatorname{Sing}(X)=\varnothing, \\
2-\operatorname{mult}_{P}(D)+\varepsilon / 2, & \text { если } Z \cap \operatorname{Sing}(X) \neq \varnothing,\end{cases}
\end{aligned}
$$

откуда следует, что $\varepsilon>2 / 3$. Но ранее мы уже показали, что $\varepsilon \leqslant 2 / 3$. Полученное противоречие завершает доказательство леммы 3.4 .

Таким образом, существует прямая $L_{1} \subset X$ такая, что $P \in L_{1}$. Положим $D=m_{1} L_{1}+\Omega$, где $\Omega$ - эффективный $\mathbb{Q}$-дивизор такой, что $L_{1} \not \subset \in \operatorname{Supp}(\Omega)$. Тогда

$$
4 / 3<1 / \omega<\Omega \cdot L_{1}=1-m_{1} L_{1} \cdot L_{1}= \begin{cases}1+m_{1}, & \text { если } L_{1} \cap \operatorname{Sing}(X)=\varnothing \\ 1+m_{1} / 2, & \text { если } L_{1} \cap \operatorname{Sing}(X) \neq \varnothing .\end{cases}
$$

СлЕДСТВИЕ 3.5. Выполнено неравенство

$$
m_{1}> \begin{cases}1 / 3, & \text { если } L_{1} \cap \operatorname{Sing}(X)=\varnothing \\ 2 / 3, & \text { если } L_{1} \cap \operatorname{Sing}(X) \neq \varnothing .\end{cases}
$$


ЗАмечаниЕ 3.6. Предположим, что поверхность $X$ является особой. Положим $O=\operatorname{Sing}(X)$. Из работы [16] следует, что $O=\Gamma_{1} \cap \Gamma_{2} \cap \Gamma_{3} \cap \Gamma_{4} \cap \Gamma_{5} \cap \Gamma_{6}$, где $\Gamma_{1}, \ldots, \Gamma_{6}-$ различные прямые на поверхности $X \subset \mathbb{P}^{3}$. Более того, выполнена эквивалентность $-2 K_{X} \sim \sum_{i=1}^{6} \Gamma_{i}$. Предположим, что $L_{1}=\Gamma_{1}$. Пусть $\Pi_{2}, \ldots, \Pi_{6} \subset \mathbb{P}^{3}$ - плоскости такие, что $L_{1} \subset \Pi_{i} \supset \Gamma_{i}$, и пусть $\Lambda_{2}, \ldots, \Lambda_{6}-$ прямые на поверхности $X$ такие, что

$$
L_{1} \cup \Gamma_{i} \cup \Lambda_{i}=\Pi_{i} \cap X \subset X \subset \mathbb{P}^{3},
$$

откуда следует, что $-K_{X} \sim L_{1}+\Gamma_{i}+\Lambda_{i}$. Тогда

$$
-5 K_{X} \sim 4 L_{1}+\sum_{i=2}^{6} \Lambda_{i}+\left(L_{1}+\sum_{i=2}^{6} \Gamma_{i}\right) \sim 4 L_{1}+\sum_{i=2}^{6} \Lambda_{i}-2 K_{X},
$$

откуда следует, что $-3 K_{X} \sim 4 L_{1}+\sum_{i=2}^{6} \Lambda_{i}$. С другой стороны, особенности лог-пары

$$
\left(X, L_{1}+\frac{\sum_{i=2}^{6} \Lambda_{i}}{3}\right)
$$

являются лог-каноническими в точке $P$. Из замечания 2.22 следует, что для завершения доказательства теоремы 3.2 мы можем считать, что

$$
\operatorname{Supp}\left(\sum_{i=2}^{6} \Lambda_{i}\right) \nsubseteq \operatorname{Supp}(D)
$$

так как $L_{1} \subseteq \operatorname{Supp}(D)$. Значит, существует прямая $\Lambda_{k}$ такая, что

$$
1=D \cdot \Lambda_{k}=\left(m_{1} L_{1}+\Omega\right) \cdot \Lambda_{k}=m_{1}+\Omega \cdot \Lambda_{k} \geqslant m_{1},
$$

поскольку $O \notin \Lambda_{k}$. Для завершения доказательства теоремы 3.2 мы можем считать, что $m_{1} \leqslant 1$, если $L_{1} \cap \operatorname{Sing}(X) \neq \varnothing$.

Из доказательства леммы 2.15 несложно вывести, что $m_{1} \leqslant 1$, если $L_{1} \cap$ $\operatorname{Sing}(X)=\varnothing$.

Лемма 3.7. Существует прямая $L_{2} \subset X$ такая, что $L_{1} \neq L_{2} u P \in L_{2}$.

ДокАЗАтельство. Предположим, что не существует прямой $L_{2} \subset X$ такой, что $L_{1} \neq L_{2}$ и $P \in L_{2}$. Тогда $T=L_{1}+C$, где $C$ - неприводимая коника на $X$ такая, что $P \in C$.

Из замечания 2.22 следует, что мы можем считать, что $C \nsubseteq \operatorname{Supp}(\Omega)$, поскольку $m_{1} \neq 0$.

Пусть $\bar{L}_{1}$ и $\bar{C}$ - собственные прообразы кривых $L_{1}$ и $C$ на $U$ соответственно. Тогда

$\bar{D} \sim_{\mathbb{Q}} m_{1} \bar{L}_{1}+\bar{\Omega} \sim_{\mathbb{Q}} \pi^{*}\left(m_{1} L_{1}+\Omega\right)-\left(m_{1}+\operatorname{mult}_{P}(\Omega)\right) E \sim_{\mathbb{Q}} \pi^{*}(D)-\operatorname{mult}_{P}(D) E$, где $\bar{\Omega}$ - собственный прообраз дивизора $\Omega$ на поверхности $U$. Имеем

$$
\begin{aligned}
0 \leqslant \bar{C} \cdot \bar{\Omega} & =2-\operatorname{mult}_{P}(D)+m_{1} \bar{C} \cdot \bar{L}<2 / 3-m_{1} \bar{C} \cdot \bar{L}_{1} \\
& =\left\{\begin{array}{l}
2 / 3-m_{1}, \quad \text { если } L_{1} \cap \operatorname{Sing}(X)=\varnothing, \\
2 / 3-m_{1} / 2, \quad \text { если } L_{1} \cap \operatorname{Sing}(X) \neq \varnothing,
\end{array}\right.
\end{aligned}
$$


откуда следует, что $m_{1}<2 / 3$, если $L_{1} \cap \operatorname{Sing}(X)=\varnothing$. Из неравенства (3.1) вытекает, что

$$
\operatorname{mult}_{Q}(\bar{\Omega})>8 / 3-\operatorname{mult}_{P}(\Omega)-m_{1}\left(1+\operatorname{mult}_{Q}\left(\bar{L}_{1}\right)\right) .
$$

Предположим, что $Q \in \bar{L}_{1}$. Тогда из леммы 2.20 следует, что

$$
\begin{aligned}
8 / 3<\bar{L}_{1} \cdot\left(\bar{\Omega}+\left(\operatorname{mult}_{P}(\Omega)+m_{1}\right) E\right) & =1-m_{1} \bar{L}_{1} \cdot \bar{L}_{1} \\
& = \begin{cases}1+2 m_{1}, & \text { если } L_{1} \cap \operatorname{Sing}(X)=\varnothing, \\
1+3 m_{1} / 2, & \text { если } L_{1} \cap \operatorname{Sing}(X) \neq \varnothing,\end{cases}
\end{aligned}
$$

что является противоречием, поскольку $m_{1} \leqslant 1$, если $L_{1} \cap \operatorname{Sing}(X) \neq \varnothing$ (см. замечание 3.6).

Итак, показано, что $Q \notin \bar{L}_{1}$. Предположим, что $Q \in \bar{C}$. Тогда

$$
2-\operatorname{mult}_{P}(\Omega)-m_{1}-m_{1} \bar{C} \cdot \bar{L} 1=\bar{C} \cdot \bar{\Omega}>8 / 3-\operatorname{mult}_{P}(\Omega)-m_{1},
$$

что является противоречием, поскольку $m_{1} \bar{C} \cdot \bar{L}_{1} \geqslant 0$. Мы видим, что $Q \notin \bar{C}$.

Существует коммутативная диаграмма

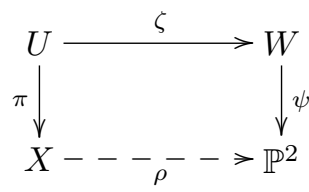

где $\zeta$ - бирациональный морфизм, который стягивает кривую $\bar{L}_{1}$, морфизм $\psi$ является двулистным накрытием, которое разветвлено над плоской кривой степени 4 , а рациональное отображение $\rho$ - линейная проекция из точки $P \in X$.

Пусть $\tau$ - бирациональная инволюция поверхности $U$, которая индуцирована двулистным накрытием $\psi$. Тогда

- инволюция $\tau$ бирегулярна $\Longleftrightarrow L_{1} \cap \operatorname{Sing}(X)=\varnothing$,

- если $L_{1} \cap \operatorname{Sing}(X) \neq \varnothing$, то инволюция $\tau$ бирегулярна на $U \backslash \bar{L}_{1}$,

- из конструкции инволюции $\tau$ следует, что $\tau(E)=\bar{C}$,

- если $L_{1} \cap \operatorname{Sing}(X)=\varnothing$, то

$$
\tau^{*}\left(\bar{L}_{1}\right) \sim \bar{L}_{1}, \quad \tau^{*}(E) \sim \bar{C}, \quad \tau^{*}\left(\pi^{*}\left(-K_{X}\right)\right) \sim \pi^{*}\left(-2 K_{X}\right)-3 E-\bar{L}_{1} .
$$

Пусть $H$ - гиперплоское сечение кубической поверхности $X$, особое в точке $\pi \circ \tau(Q) \in C$. Тогда $P \notin H$, поскольку кривая $C$ неособа. Пусть $\bar{H}-$ собственный прообраз кривой $H$ на поверхности $U$. Тогда $\bar{L}_{1} \nsubseteq \operatorname{Supp}(\bar{H}) \nsupseteq \bar{C}$.

Положим $\bar{R}=\tau(\bar{H})$ и $R=\pi(\bar{R})$. Кривая $\bar{R}$ имеет особенность в точке $Q$, и

$$
\bar{R} \sim \pi^{*}\left(-2 K_{X}\right)-3 E-\bar{L}_{1},
$$

поскольку $R$ не проходит через особую точку поверхности $X$ при $\operatorname{Sing}(X) \neq \varnothing$.

Предположим, что $R$ неприводима. Тогда $R+L_{1} \sim-2 K_{X}$, но лог-пара $\left(X, \frac{3}{8}\left(R+L_{1}\right)\right)$ лог-канонична. Значит (см. замечание 2.22$)$, мы можем считать, что $R \nsubseteq \operatorname{Supp}(D)$. Тогда

$$
\begin{aligned}
& 5-2\left(m_{1}+\operatorname{mult}_{P}(\Omega)\right)+m_{1}\left(1+\bar{L}_{1} \cdot \bar{L}_{1}\right) \\
& \quad=\bar{R} \cdot \bar{\Omega} \geqslant 2 \operatorname{mult}_{Q}(\bar{\Omega})>2\left(8 / 3-m_{1}-\operatorname{mult}_{P}(\Omega)\right),
\end{aligned}
$$

откуда следует, что $m_{1}<0$. Противоречие. Показано, что $R$ приводима. 
Из приводимости кривой $R$ сразу следует, что существует прямая $L \subset X$ такая, что $P \notin L$ и $\pi \circ \tau(Q) \in L$. Тогда $L \cap L_{1}=\varnothing$, поскольку $\pi \circ \tau(Q) \in C$ и $\left(C+L_{1}\right) \cdot L=T \cdot L=1$. Значит, существует единственная коника $Z \subset X$ такая, что $-K_{X} \sim L+Z$ и $P \in Z$. Коника $Z$ неприводима и $P=Z \cap L_{1}$, поскольку $(L+Z) \cdot L_{1}=1$.

Пусть $\bar{L}$ и $\bar{Z}$ - собственные прообразы кривых $L$ и $Z$ на $U$ соответственно. Тогда

$$
\begin{array}{ll}
\bar{L} \cdot \bar{C}=\bar{Z} \cdot E=1, & \bar{L}_{1} \cdot \bar{Z}=\bar{L} \cdot E=\bar{L} \cdot \bar{L}_{1}=0, \\
\bar{Z} \cdot \bar{Z}=1-\bar{L} \cdot \bar{Z}, & \bar{L} \cdot \bar{Z}= \begin{cases}2, & \text { если } L \cap \operatorname{Sing}(X)=\varnothing, \\
3 / 2, & \text { если } L \cap \operatorname{Sing}(X) \neq \varnothing .\end{cases}
\end{array}
$$

По построению $\tau(\bar{Z})=\bar{L}$. Тогда $Q \in \bar{Z}$. Предположим, что $\bar{Z} \nsubseteq \operatorname{Supp}(\bar{\Omega})$. Тогда

$$
2-m_{1}-\operatorname{mult}_{P}(\Omega)=\bar{Z} \cdot \bar{\Omega}>8 / 3-m_{1}-\operatorname{mult}_{P}(\Omega),
$$

что является противоречием. Показано, что $\bar{Z} \subseteq \operatorname{Supp}(\bar{\Omega})$. Лог-пара $(X$, $\omega(L+Z))$ имеет лог-канонические особенности в точке $P$. Таким образом, мы можем считать, что $\bar{L} \nsubseteq \operatorname{Supp}(\bar{\Omega})$ (см. замечание 2.22$)$. Положим $D=$ $\varepsilon Z+m_{1} L_{1}+\Upsilon$, где $\Upsilon$ - эффективный $\mathbb{Q}$-дивизор такой, что $Z \not \subset \operatorname{Supp}(\Upsilon) \not \supset L_{1}$. Тогда

$$
\begin{aligned}
1=L \cdot D & =\varepsilon L \cdot Z+m_{1} L \cdot L_{1}+L \cdot \Upsilon=\varepsilon L \cdot Z+L \cdot \Upsilon \geqslant \varepsilon L \cdot Z \\
& = \begin{cases}2 \varepsilon, & \text { если } L \cap \operatorname{Sing}(X)=\varnothing, \\
3 \varepsilon / 2, & \text { если } L \cap \operatorname{Sing}(X) \neq \varnothing,\end{cases}
\end{aligned}
$$

откуда следует, что $\varepsilon \leqslant 2 / 3$. Но $\bar{Z} \cap \bar{L}_{1}=\varnothing$. Поэтому, из леммы 2.20 следует, что

$$
2-\operatorname{mult}_{P}(D)-\varepsilon \bar{Z} \cdot \bar{Z}=\bar{Z} \cdot \bar{\Upsilon}>8 / 3-\operatorname{mult}_{P}(D),
$$

где $\bar{\Upsilon}$ - собственный прообраз дивизора $\Upsilon$ на поверхности $U$. Мы видим, что $\varepsilon>2 / 3$, но $\varepsilon \leqslant 2 / 3$. Полученное противоречие завершает доказательство леммы 3.7 .

Итак, показано, что $T=L_{1}+L_{2}+L_{3}$, где $L_{3}-$ прямая такая, что $P \notin L_{3}$. Положим $D=m_{1} L_{1}+m_{2} L_{2}+\Delta$, где $\Delta$ - эффективный $\mathbb{Q}$-дивизор такой, что $L_{1} \nsubseteq \operatorname{Supp}(\Delta) \nsupseteq L_{2}$.

Отметим, что, согласно следствию 3.5 , выполнены неравенства $m_{1}>1 / 3$ и $m_{2}>1 / 3$. Значит, по замечанию 2.22 мы можем считать, что $L_{3} \not \subset \operatorname{Supp}(\Delta)$. Если на $L_{1}$ или $L_{2}$ лежит особая точка поверхности $X$, то без ограничения общности можно считать, что она лежит на $L_{1}$. Таким образом, $L_{3} \cdot L_{2}=1$ и $L_{3} \cdot L_{1}=1 / 2$ в случае, когда $L_{1} \cap \operatorname{Sing}(X) \neq \varnothing$. Аналогично, мы видим, что $L_{3} \cdot L_{2}=L_{3} \cdot L_{1}=1$ в случае, когда $L_{1} \cap \operatorname{Sing}(X)=\varnothing$. Тогда $1-m_{1} L_{1} \cdot L_{3}-m_{2}=$ $L_{3} \cdot \Delta \geqslant 0$.

Пусть $\bar{L}_{1}$ и $\bar{L}_{3}$ - собственные прообразы прямых $L_{1}$ и $L_{2}$ на $U$ соответственно. Тогда

$$
m_{1} \bar{L}_{1}+m_{2} \bar{L}_{2}+\bar{\Delta} \sim_{\mathbb{Q}} \pi^{*}\left(m_{1} L_{1}+m_{2} L_{2}+\Delta\right)-\left(m_{1}+m_{2}+\operatorname{mult}_{P}(\Delta)\right) E,
$$


где $\bar{\Delta}$ - собственный прообраз дивизора $\Delta$ на $U$. Из неравенства (3.1) следует, что

$$
\operatorname{mult}_{Q}(\bar{\Delta})>8 / 3-\operatorname{mult}_{P}(\Delta)-m_{1}\left(1+\operatorname{mult}_{Q}\left(\bar{L}_{1}\right)\right)-m_{1}\left(1+\operatorname{mult}_{Q}\left(\bar{L}_{2}\right)\right) .
$$

Лемма 3.8. Кривая $\bar{L}_{2}$ не содержит точку $Q$.

ДокАЗАтЕЛьство. Предположим, что $Q \in \bar{L}_{2}$. Тогда

$$
1-\operatorname{mult}_{P}(\Delta)-m_{1}+m_{2}=\bar{L}_{2} \cdot \bar{\Delta}>8 / 3-\operatorname{mult}_{P}(\Delta)-m_{1}-m_{2}
$$

по лемме 2.20. Значит, $m_{2}>5 / 6$. С другой стороны, из леммы 2.20 следует, что

$$
1-m_{2}-m_{1} L_{1} \cdot L_{1}=\Delta \cdot L_{1}>4 / 3-m_{2} .
$$

Но $L_{1} \cdot L_{1}=-1$, если $L_{1} \cap \operatorname{Sing}(X)=\varnothing$, и $L_{1} \cdot L_{1}=-1 / 2$, если $L_{1} \cap \operatorname{Sing}(X) \neq \varnothing$. Тогда по следствию 3.5

$$
m_{1}> \begin{cases}1 / 3, & \text { если } L_{1} \cap \operatorname{Sing}(X)=\varnothing, \\ 2 / 3, & \text { если } L_{1} \cap \operatorname{Sing}(X) \neq \varnothing,\end{cases}
$$

что невозможно, поскольку $m_{2}>5 / 6$ и $1>m_{1} L_{1} \cdot L_{3}+m_{2}$.

Лемма 3.9. Кривая $\bar{L}_{1}$ не содержит точку $Q$.

ДокАЗАТЕльство. Предположим, что $Q \in \bar{L}_{1}$. Рассуждая как в доказательстве леммы 3.8 , мы видим, что $L_{1} \cap \operatorname{Sing}(X) \neq \varnothing$, откуда $\bar{L}_{1} \cdot \bar{L}_{1}=-1 / 2$. Тогда $m_{1}>10 / 9$, так как

$$
1+3 m_{1} / 2=\bar{L}_{2} \cdot\left(\bar{\Delta}+\left(\operatorname{mult}_{P}(\Delta)-m_{1}-m_{2}\right) E\right)>8 / 3
$$

по лемме 2.20. С другой стороны, по замечанию 3.6 выполнено неравенство $m_{1} \leqslant 1$. Полученное противоречие завершает доказательство леммы.

Итак, показано, что $\bar{L}_{1} \not \ngtr Q \notin \bar{L}_{2}$. Существует коммутативная диаграмма

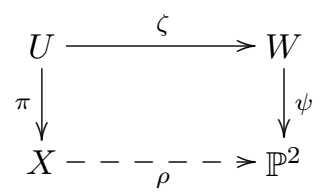

где $\zeta$ - бирациональный морфизм, который стягивает кривые $\bar{L}_{1}$ и $\bar{L}_{2}$, морфизм $\psi$ является двулистным накрытием, которое разветвлено над плоской кривой степени 4 , а рациональное отображение $\rho$ является линейной проекцией из точки $P$.

Пусть $\tau$ - бирациональная инволюция поверхности $U$, которая индуцирована двулистным накрытием $\psi$. Тогда

- инволюция $\tau$ бирегулярна $\Longleftrightarrow L_{1} \cap \operatorname{Sing}(X)=\varnothing$,

- если $L_{1} \cap \operatorname{Sing}(X) \neq \varnothing$, то инволюция $\tau$ бирегулярна на $U \backslash \bar{L}_{1}$,

- из конструкции инволюции $\tau$ следует, что $\tau\left(\bar{L}_{2}\right)=\bar{L}_{2}$,

- если $L_{1} \cap \operatorname{Sing}(X)=\varnothing$, то $\tau\left(\bar{L}_{1}\right)=\bar{L}_{1}$ и

$$
\tau^{*}\left(\pi^{*}\left(-K_{X}\right)\right) \sim \pi^{*}\left(-2 K_{X}\right)-3 E-\bar{L}_{1}-\bar{L}_{2} .
$$


Обозначим через $\bar{L}_{3}$ собственный прообраз прямой $L_{3}$ на поверхности $U$. Тогда $\tau(E)=\bar{L}_{3}$ и

$$
L_{1} \cup L_{2} \not \supset \pi \circ \tau(Q) \in L_{3} .
$$

Лемма 3.10. Прямая $L_{3}$ является единственной прямой на $X$, которая проходит через точку $\pi \circ \tau(Q)$.

ДокАЗАТЕЛЬство. Предположим, что существует прямая $L \subset X$ такая, что $L \neq L_{3}$ и $\pi \circ \tau(Q) \in L$. Тогда $L \cap L_{1}=L \cap L_{2}=\varnothing$, поскольку $\pi \circ \tau(Q) \in L_{3}$ и $\left(L_{1}+L_{2}+L_{3}\right) \cdot L=1$. Значит, существует единственная коника $Z \subset X$ такая, что $-K_{X} \sim L+Z$ и $P \in Z$. Коника $Z$ неприводима, поскольку $P \notin L$ и $P$ не является точкой Эккарда.

Пусть $\bar{L}$ и $\bar{Z}$ - собственные прообразы кривых $L$ и $Z$ на $U$ соответственно. Тогда

$$
\bar{L} \cdot \bar{L}_{3}=\bar{Z} \cdot E=1, \quad \bar{Z} \cdot \bar{Z}=1-\bar{L} \cdot \bar{Z}, \quad \bar{L} \cdot \bar{Z}= \begin{cases}2, & \text { если } L \cap \operatorname{Sing}(X)=\varnothing, \\ 3 / 2, & \text { если } L \cap \operatorname{Sing}(X) \neq \varnothing,\end{cases}
$$

и $\bar{L}_{1} \cdot \bar{Z}=\bar{L}_{2} \cdot \bar{Z}=\bar{L} \cdot E=\bar{L} \cdot \bar{L}_{1}=\bar{L} \cdot \bar{L}_{2}=0$. Из конструкции инволюции $\tau$ следует, что $\tau(\bar{Z})=\bar{L}$. Тогда $Q \in \bar{Z}$, откуда следует, что $\bar{Z} \subseteq \operatorname{Supp}(\bar{\Delta})$, поскольку

$$
2-\operatorname{mult}_{P}(\Delta)-m_{1}-m_{2}=\bar{Z} \cdot \bar{\Omega}>8 / 3-\operatorname{mult}_{P}(\Delta)-m_{1}-m_{2}
$$

в случае, когда $\bar{Z} \nsubseteq \operatorname{Supp}(\bar{\Delta})$. С другой стороны, лог-пара $(X, \omega(L+Z))$ имеет лог-канонические особенности в точке $P$. Таким образом, согласно замечанию 2.22 , можно считать, что $\bar{L} \nsubseteq \operatorname{Supp}(\bar{\Delta})$. Положим $D=\varepsilon Z+m_{1} L_{1}+$ $m_{2} L_{2}+\Upsilon$, где $\Upsilon$ - эффективный $\mathbb{Q}$-дивизор такой, что $Z \nsubseteq \operatorname{Supp}(\Upsilon)$. Тогда

$$
\begin{aligned}
1=L \cdot D & =\varepsilon L \cdot Z+m_{1} L \cdot L_{1}+L \cdot \Upsilon=\varepsilon L \cdot Z+L \cdot \Upsilon \geqslant \varepsilon L \cdot Z \\
& = \begin{cases}2 \varepsilon, & \text { если } L \cap \operatorname{Sing}(X)=\varnothing, \\
3 \varepsilon / 2, & \text { если } L \cap \operatorname{Sing}(X) \neq \varnothing,\end{cases}
\end{aligned}
$$

откуда следует, что выполнено неравенство $\varepsilon \leqslant 2 / 3$. С другой стороны, мы знаем, что $\bar{Z} \cap \bar{L}_{1}=\varnothing$. Таким образом, из леммы 2.20 следует, что

$$
2-\operatorname{mult}_{P}(D)-\varepsilon \bar{Z} \cdot \bar{Z}=\bar{Z} \cdot \bar{\Upsilon}>8 / 3-\operatorname{mult}_{P}(D),
$$

где $\bar{\Upsilon}$ - собственный прообраз дивизора $\Upsilon$ на $U$. Мы видим, что $\varepsilon>2 / 3$, но ранее было показано, что $\varepsilon \leqslant 2 / 3$. Противоречие. Утверждение леммы доказано.

Итак, существует единственная неприводимая коника $C \subset X$ такая, что $-K_{X} \sim L_{3}+C$ и $\pi \circ \tau(Q) \in C$. Тогда $C+L_{3}$ - гиперплоское сечение поверхности $X$, которое имеет особенность в точке $\pi \circ \tau(Q)$. Пусть $\bar{C}$ - собственный прообраз коники $C$ на $U$. Положим $\bar{Z}=\tau(\bar{C})$ и $Z=\pi(\bar{Z})$.

ЛЕмма 3.11. Выполнено неравенство $L_{1} \cap \operatorname{Sing}(X) \neq \varnothing$. 
ДоказАтЕЛЬСтво. Предположим, что $L_{1} \cap \operatorname{sing}(X)=\varnothing$. Тогда $C \cap L_{1}=$ $C \cap L_{2}=\varnothing$, поскольку $\left(L_{1}+L_{2}+L_{3}\right) \cdot C=L_{3} \cdot C=2$. Легко видеть, что $\bar{Z} \sim \pi^{*}\left(-2 K_{X}\right)-4 E-\bar{L}_{1}-\bar{L}_{2}$, а кривая $Z$ имеет особенность в точке $P$. Тогда $-2 K_{X} \sim Z+L_{1}+L_{2}$, но особенности лог-пары $\left(U, \frac{1}{2}\left(Z+L_{1}+L_{2}\right)\right)$ логканонические в точке $P$. Значит (см. замечание 2.22 ), мы можем считать, что $Z \nsubseteq \operatorname{Supp}(D)$. По построению $Q \in \bar{Z}$ и $\bar{Z} \cdot E=2$. Из неравенства (3.1) следует, что

$$
4-2 \operatorname{mult}_{P}(D)=\bar{Z} \cdot \bar{D} \geqslant \operatorname{mult}_{Q}(\bar{D})>8 / 3-\operatorname{mult}_{P}(D),
$$

откуда $\operatorname{mult}_{P}(D)<4 / 3$, что невозможно, поскольку $\operatorname{mult}_{P}(D)>4 / 3$. Лемма доказана.

Итак, показано, что $L_{1} \cap L_{3}=\operatorname{Sing}(X) \neq \varnothing$. Тогда $L_{1} \cap L_{2} \in C$, откуда следует, что

$$
\bar{Z} \sim \pi^{*}\left(-2 K_{X}\right)-4 E-2 \bar{L}_{1}-\bar{L}_{2},
$$

а $Z$ - гладкая рациональная кубика. Тогда $-2 K_{X} \sim Z+2 L_{1}+L_{2}$, но особенности лог-пары $\left(U, \frac{1}{2}\left(Z+2 L_{1}+L_{2}\right)\right)$ лог-канонические в точке $P$. Значит, можно считать, что $Z \nsubseteq \operatorname{Supp}(D)$ (см. замечание 2.22 ). Имеем $Q \in \bar{Z}$ и $\bar{Z} \cdot E=\bar{L}_{1}=1$. Из неравенства (3.1) получаем

$$
3-\operatorname{mult}_{P}(\Delta)-2 m_{1}-m_{2}=\bar{Z} \cdot \bar{\Delta} \geqslant \operatorname{mult}_{Q}(\bar{\Delta})>8 / 3-\operatorname{mult}_{P}(\Delta)-m_{1}-m_{2},
$$

откуда следует, что $m_{1}<1 / 3$. С другой стороны, $m_{1}>2 / 3$ по следствию 3.5 . Полученное противоречие завершает доказательство теоремы 3.2 .

\section{4. Поверхности дель Пеццо}

Пусть $X$ - поверхность дель Пеццо, которая имеет не более чем канонические особенности, пусть $O$ - некоторая точка поверхности $X$, и пусть $B_{X}$ - эффективный $\mathbb{Q}$-дивизор на поверхности $X$. Предположим, что точка $O$ является либо гладкой, либо обыкновенной двойной точкой поверхности $X$, а поверхность $X$ неособа вне точки $O \in X$.

Лемма 4.1. Предположим, ито $\operatorname{Sing}(X)=O, K_{X}^{2}=2 u B_{X} \sim_{\mathbb{Q}}-\mu K_{X}$, әде $0<\mu<2 / 3$. Тогда $\mathbb{L} \mathbb{C S}\left(X, \mu B_{X}\right)=\varnothing$.

ДокАзАтЕЛЬСтво. Предположим, что $\mathbb{L} \mathbb{C S}\left(X, \mu B_{X}\right) \neq \varnothing$. Тогда существует кривая $\mathbb{P}^{1} \cong L \subset X$ такая, что $\operatorname{LCS}\left(X, \mu B_{X}\right) \nsubseteq L L$, выполнено равенство $L \cdot L=-1$ и $L \cap \operatorname{Sing}(X)=\varnothing$. Следовательно, существует бирациональный морфизм $\pi: X \rightarrow S$, который стягивает кривую $L$. Тогда $\mathbb{L} \mathbb{C} S\left(S, \mu \pi\left(B_{X}\right)\right) \neq \varnothing$ в силу выбора кривой $L \subset X$. С другой стороны, выполнена эквивалентность $-K_{S} \sim_{\mathbb{Q}} \pi\left(B_{X}\right)$, а поверхность $S$ является кубической поверхностью в $\mathbb{P}^{3}$, которая имеет не более чем одну обыкновенную двойную точку, что является противоречием (см. примеры 1.11 и 1.10 ).

Лемма 4.2. Предположим, что $\operatorname{Sing}(X)=\varnothing$ и выполнены равенство $K_{X}^{2}=5$ и эквивалентность $B_{X} \sim_{\mathbb{Q}}-\mu K_{X}$, где $\mu \in \mathbb{Q}$ таково, что $0<\mu<2 / 3$. Предположим, что $\mathbb{L} \mathbb{C S}\left(X, B_{X}\right) \neq \varnothing$. Тогда либо множество $\mathbb{L} \mathbb{C S}\left(X, B_{X}\right)$ содержит кривую, либо существуют кривая $\mathbb{P}^{1} \cong L \subset X$ и точка $P \in L$ maкие, ито $L \cdot L=-1 u \operatorname{LCS}\left(X, B_{X}\right)=P$. 
ДоказАтельСтво. Предположим, что $\mathbb{L} \mathbb{C} S\left(X, B_{X}\right)$ не содержит кривых. Тогда из теоремы 2.7 следует, что $\operatorname{LCS}\left(X, B_{X}\right)=P$ для некоторой точки $P \in X$. Можно считать, что точка $P$ не содержится ни в одной кривой $\mathbb{P}^{1} \cong L \subset X$ такой, что $L \cdot L=-1$. Тогда существует бирациональный морфизм $\varphi: X \rightarrow \mathbb{P}^{2}$, который является изоморфизмом в окрестности точки $P$. При этом $\varphi(P) \in$ $\operatorname{LCS}\left(\mathbb{P}^{2}, \varphi\left(B_{X}\right)\right)$, множество $\mathbb{L} \mathbb{C} S\left(\mathbb{P}^{2}, \varphi\left(B_{X}\right)\right)$ не содержит кривых и выполнена эквивалентность $\varphi\left(B_{X}\right) \sim_{\mathbb{Q}}-\mu K_{\mathbb{P}^{2}}$, что сразу приводит к противоречию с леммой 2.8 , поскольку $\mu<2 / 3$.

Пример 4.3. Предположим, что $O=\operatorname{Sing}(X)$ и $K_{X}^{2}=5$. Пусть $\alpha: V \rightarrow X-$ раздутие точки $O$, и пусть $E$ - исключительный дивизор раздутия $\alpha$. Тогда существует бирациональный морфизм $\omega: V \rightarrow \mathbb{P}^{2}$ такой, что морфизм $\omega$ стягивает кривые $E_{1}, E_{2}, E_{3}, E_{4}$, а кривая $\omega(E)$ является прямой в $\mathbb{P}^{2}$, которая содержит точки $\omega\left(E_{1}\right), \omega\left(E_{2}\right), \omega\left(E_{3}\right)$, но $\omega(E) \not \supset \omega\left(E_{4}\right)$.

Пусть $Z$ - прямая в $\mathbb{P}^{2}$ такая, что $\omega\left(E_{1}\right) \in Z \ni \omega\left(E_{4}\right)$. Тогда

$$
2 E+\bar{Z}+2 E_{1}+E_{2}+E_{3} \sim-K_{V},
$$

где $\bar{Z}$ - собственный прообраз прямой $Z$ на поверхности $V$. Имеем

$$
\operatorname{lct}\left(X, \alpha(\bar{Z})+2 \alpha\left(E_{1}\right)+\alpha\left(E_{2}\right)+\alpha\left(E_{3}\right)\right)=1 / 2,
$$

откуда следует, что $\operatorname{lct}(X) \leqslant 1 / 2$. Предположим, что $-K_{X} \sim_{\mathbb{Q}} 2 B_{X}$, но особенности лог-пары $\left(X, B_{X}\right)$ не являются лог-каноническими. Тогда

$$
K_{V}+B_{V}+m E \sim_{\mathbb{Q}} \alpha^{*}\left(K_{X}+B_{X}\right)
$$

для некоторого $m \geqslant 0$, где $B_{V}$ - собственный прообраз дивизора $B_{X}$ на поверхности $V$. Следовательно, особенности лог-пары $\left(V, B_{V}+m E\right)$ не являются лог-каноническими в некоторой точке $P \in V$. Существует бирациональный морфизм $\pi: V \rightarrow U$, являющийся изоморфизмом в окрестности точки $P \in X$, такой, что поверхность $U$ является гладкой поверхностью дель Пеццо и $K_{U}^{2}=6$, откуда следует, что лог-пара $\left(U, \pi\left(B_{V}\right)+m \pi(E)\right)$ не лог-канонична в точке $\pi(P)$. С другой стороны, выполнена эквивалентность $\pi\left(B_{V}\right)+m \pi(E) \sim_{\mathbb{Q}}-(1 / 2) K_{U}$, что является противоречием, поскольку $\operatorname{lct}(U)=1 / 2$ (см. пример 1.10). Итак, показано, что $\operatorname{lct}(X)=1 / 2$.

ПримеР 4.4. Предположим, что $K_{X}^{2}=4$. Принимая во внимание рассуждения в примере 4.3, мы видим, что

$$
\operatorname{lct}(X)= \begin{cases}1 / 2, & \text { если } O=\operatorname{Sing}(X), \\ 2 / 3, & \text { если } \operatorname{Sing}(X)=\varnothing .\end{cases}
$$

Предположим, что $B_{X} \sim_{\mathbb{Q}}-K_{X}$, но особенности лог-пары $\left(X, \lambda B_{X}\right)$ не являются лог-каноническими в некоторой точке $P \in X \backslash O$. Существует коммутативная диаграмма

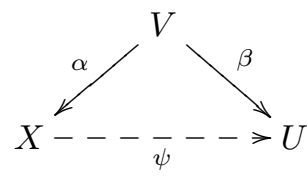


где $U$ - кубическая поверхность в $\mathbb{P}^{3}$, которая имеет не более чем канонические особенности, морфизм $\alpha$ является раздутием точки $P$, морфизм $\beta$ бирационален, а рациональное отображение $\psi$ является линейной проекцией из точки $P \in X$. Тогда

$$
K_{V}+\lambda B_{V}+\left(\lambda \operatorname{mult}_{P}\left(B_{X}\right)-1\right) E \sim_{\mathbb{Q}} \alpha^{*}\left(K_{X}+\lambda B_{X}\right),
$$

где $E$ - исключительный дивизор раздутия $\alpha$, а $B_{V}-$ собственный прообраз дивизора $B_{X}$ на поверхности $V$. Особенности лог-пары

$$
\left(V, \lambda B_{V}+\left(\lambda \operatorname{mult}_{P}\left(B_{X}\right)-1\right) E\right)
$$

не являются лог-каноническими в некоторой точке $Q \in E$, и выполнено неравенство $\operatorname{mult}_{P}\left(B_{X}\right)>1 / \lambda$. Тогда особенности лог-пары

$$
\left(V, \lambda B_{V}+\left(\lambda \operatorname{mult}_{P}\left(B_{X}\right)-\lambda\right) E\right)
$$

также не являются лог-каноническими в точке $Q \in E$, но выполнена эквивалентность

$$
B_{V}+\left(\operatorname{mult}_{P}\left(B_{X}\right)-1\right) E \sim_{\mathbb{Q}}-K_{V}+\alpha^{*}\left(K_{X}+B_{X}\right) \sim_{\mathbb{Q}}-K_{V} .
$$

Предположим, что $P$ не лежит ни на одной прямой, содержащейся в $X$. Тогда

- морфизм $\beta: V \rightarrow U$ является изоморфизмом,

- кубическая поверхность $U$ неособа вне точки $\psi(O)$,

- точка $\psi(O)$ является обыкновенной двойной точкой поверхности $U$, откуда следует, что выполнено неравенство $\lambda>2 / 3$ (см. пример 1.11).

Предположим, что $\lambda=3 / 4$. Тогда по теореме 3.2 точка $\psi(Q) \in U \subset \mathbb{P}^{3}$ обязана быть точкой Эккарда поверхности $U$ (см. определение 3.1 ). С другой стороны, кривая $\beta(E) \subset U$ является прямой. Таким образом, поверхность $X$ содержит две неприводимые коники $C_{1} \neq C_{2}$ такие, что $P=C_{1} \cap C_{2}$ и $C_{1}+$ $C_{2} \sim-K_{X}$

Лемма 4.5. Предположим, что $O=\operatorname{Sing}(X), K_{X}^{2}=6$ и существует диаграмма

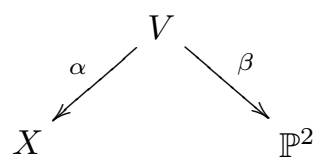

где $\beta$ - раздутие трех различных точек $P_{1}, P_{2}, P_{3} \in \mathbb{P}^{2}$, которые лежат на одной прямой $L \subset \mathbb{P}^{2}$, а $\alpha$ - бирациональный морфизм, который стягивает неприводимую кривую $\bar{L}$ в точку $O$ такую, что $\beta(\bar{L})=L$. Тогда $\operatorname{LCS}\left(X, \lambda B_{X}\right)=O$ в случае, когда $\operatorname{LCS}\left(X, \lambda B_{X}\right) \neq \varnothing, B_{X} \sim_{\mathbb{Q}}-K_{X} u \lambda<1 / 2$.

ДокАЗАтельСтво. Предположим, что $\varnothing \neq \operatorname{LCS}\left(X, \lambda B_{X}\right) \neq O$, но $B_{X} \sim_{\mathbb{Q}}$ $-K_{X}$. Пусть $M$ - общая прямая в $\mathbb{P}^{2}$, и пусть $\bar{M}-$ собственный прообраз прямой $M$ на поверхности $V$. Тогда $-K_{X} \sim 2 \alpha(\bar{M})$ и $O \in \alpha(\bar{M})$. Значит, множество $\mathbb{L} \mathbb{C} S\left(X, \lambda B_{X}\right)$ содержит кривую, поскольку в противном случае $\operatorname{LCS}\left(X, \lambda B_{X}+\alpha(\bar{M})\right)$ было бы несвязно, что невозможно по теореме 2.7. 
Пусть $C$ - неприводимая кривая на поверхности $X$ такая, что $C \subseteq \operatorname{LCS}(X$, $\left.\lambda B_{X}\right)$. Тогда $B_{X}=\varepsilon C+\Omega$, где $\varepsilon>2$, а $\Omega$ - эффективный $\mathbb{Q}$-дивизор такой, что $C \not \subset \operatorname{Supp}(\Omega)$.

Пусть $\Gamma_{i}$ - собственный прообраз на поверхности $X$ достаточно общей прямой в $\mathbb{P}^{2}$, которая проходит через точку $P_{i}$. Тогда $O \notin \Gamma_{1} \cup \Gamma_{2} \cup \Gamma_{3}$ и $-K_{X} \cdot \Gamma_{1}=$ $-K_{X} \cdot \Gamma_{2}=-K_{X} \cdot \Gamma_{3}=2$. С другой стороны, выполнена эквивалентность $-K_{X} \sim_{\mathbb{Q}} \Gamma_{1}+\Gamma_{2}+\Gamma_{3}$, откуда сразу следует, что $C \cdot \Gamma_{m} \neq 0$ для некоторого $m \in\{1,2,3\}$. Тогда

$$
2=B_{X} \cdot \Gamma_{m}=(\varepsilon C+\Omega) \cdot \Gamma_{m} \geqslant \varepsilon C \cdot \Gamma_{m} \geqslant \varepsilon>2,
$$

поскольку $\Gamma_{m} \not \subset \operatorname{Supp}\left(B_{X}\right)$. Полученное противоречие завершает доказательство леммы.

ЗАмечАНИЕ 4.6. Предположим, что $O=\operatorname{Sing}(X)$ и $K_{X}^{2}=6$. Пусть отображение $\alpha: V \rightarrow X$ является раздутием точки $O \in X$, и пусть $E$ - исключительный дивизор раздутия $\alpha$. Тогда

$$
K_{V}+B_{V}+m E \sim_{\mathbb{Q}} \alpha^{*}\left(K_{X}+B_{X}\right)
$$

для некоторого $m \geqslant 0$, где $B_{V}$ - собственный прообраз дивизора $B_{X}$ на поверхности $V$. Заметим, что $\operatorname{lct}(X) \leqslant 1 / 3$. Предположим, что $\operatorname{lct}(X)<1 / 3$, т. е. найдется эффективный $\mathbb{Q}$-дивизор $B_{X} \sim_{\mathbb{Q}}-K_{X}$ такой, что особенности логпары $\left(X, \frac{1}{3} B_{X}\right)$ не являются лог-каноническими. Тогда особенности лог-пары $\left(V, \frac{1}{3}\left(B_{V}+m E\right)\right)$ не являются лог-каноническими в некоторой точке $P \in V$. Существует бирациональный морфизм $\pi: V \rightarrow U$ такой, что либо $U \cong \mathbb{F}_{1}$, либо $U \cong \mathbb{P}^{1} \times \mathbb{P}^{1}$, а бирациональный морфизм $\pi$ является изоморфизмом в окрестности точки $P \in X$. Тогда особенности лог-пары $\left(U, \frac{1}{3}\left(\pi\left(B_{V}\right)+m \pi(E)\right)\right)$ не являются лог-каноническими в точке $\pi(P)$. С другой стороны, выполнена эквивалентность $-K_{U} \sim_{\mathbb{Q}} \pi\left(B_{V}\right)+m \pi(E)$, откуда сразу следует противоречие с примером 1.10. Таким образом, мы видим, что $\operatorname{lct}(X)=1 / 3$.

ЛЕмма 4.7. Предположим, что $X \cong \mathbb{P}(1,1,2)$, выполнена эквивалентность $B_{X} \sim_{\mathbb{Q}}-K_{X}$ и существует точка $P \in X$ такая, что $O \neq P \in \operatorname{LCS}\left(X, \lambda B_{X}\right)$ для некоторого неотрицательного рационального числа $\lambda<1 / 2$. Пусть $L-$ единственная кривая в линейной системе $\left|\mathscr{O}_{\mathbb{P}(1,1,2)}(1)\right|$ maкая, что $P \in L$. Тогда $L \subseteq \operatorname{LCS}\left(X, \lambda B_{X}\right)$.

ДокАзАтельство. Предположим, что существует кривая $\Gamma \in \operatorname{LCS}\left(X, \lambda B_{X}\right)$ такая, что $P \in \Gamma \neq L$. Тогда $B_{X}=\mu \Gamma+\Omega$, где $\mu>2$, а $\Omega$ - эффективный $\mathbb{Q}$-дивизор такой, что $\Gamma \not \subset \operatorname{Supp}(\Omega)$. Следовательно, $\mu \Gamma+\Omega \sim_{\mathbb{Q}} 4 L$ и выполнена эквивалентность $\Gamma \sim m L$, где $m \in \mathbb{Z}_{>0}$. С другой стороны, поскольку $P \in$ $\Gamma \neq L$, то выполнено неравенство $m \geqslant 2$, откуда сразу следует противоречие.

Предположим, что $L \nsubseteq \mathbb{L C S}\left(X, \lambda B_{X}\right)$. Тогда из теоремы 2.7 следует, что $\operatorname{LCS}\left(X, \lambda B_{X}\right)=P$, поскольку множество $\mathbb{L} \mathbb{C S}\left(X, \lambda B_{X}\right)$ не содержит кривых, которые проходят через $P$.

Пусть $C$ - общая кривая в линейной системе $\left|\mathscr{O}_{\mathbb{P}(1,1,2)}(1)\right|$. Тогда $\operatorname{LCS}(X$, $\left.\lambda B_{X}+C\right)=P \cup C$, что противоречит теореме 2.7 . 
Лемма 4.8. Предположим, что $X \cong \mathbb{F}_{1}$. Тогда существуют $0 \leqslant \mu \in \mathbb{Q} \ni$ $\lambda \geqslant 0$ такие, что выполнено соотношение $B_{X} \sim_{\mathbb{Q}} \mu C+\lambda L$, где $C$ u $L-$ неприводимые кривые на $X$ такие, что $C \cdot C=-1, C \cdot L=1 u L \cdot L=0$. Предположим, что $\mu<1$ и $\lambda<1$. Тогда $\mathbb{L} \mathbb{C} S\left(X, B_{X}\right)=\varnothing$.

ДоказАтельство. Очевидно, что множество $\mathbb{L} \mathbb{C} S\left(X, B_{X}\right)$ не содержит кривых, поскольку кривые $L$ и $C$ порождают конус эффективных дивизоров поверхности $X$. Предположим, что множество $\mathbb{L} \mathbb{C} S\left(X, B_{X}\right)$ содержит точку $O \in X$. Тогда

$$
K_{X}+B_{X}+((1-\mu) C+(2-\lambda) L) \sim_{\mathbb{Q}}-(L+C)
$$

так как $-K_{X} \sim_{\mathbb{Q}} 2 C+3 L$. С другой стороны, из теоремы 2.6 следует, что отображение

$$
0=H^{0}\left(\mathscr{O}_{X}(-L-C)\right) \rightarrow H^{0}\left(\mathscr{O}_{\mathscr{L}}\left(X, B_{X}\right)\right) \neq 0
$$

сюръективно, поскольку дивизор $(1-\mu) C+(2-\lambda) L$ обилен. Противоречие.

Лемма 4.9. Предположим, что $\operatorname{Sing}(X)=\varnothing u K_{X}^{2}=7$. Тогда

$$
L_{1} \cdot L_{1}=L_{2} \cdot L_{2}=L_{3} \cdot L_{3}=-1, \quad L_{1} \cdot L_{2}=L_{2} \cdot L_{3}=1, \quad L_{1} \cdot L_{3}=0,
$$

где $L_{1}, L_{2}, L_{3}$ - исключителъные кривые на поверхности $X$. Предположим, что $\operatorname{LCS}\left(X, B_{X}\right) \neq \varnothing$, но $B_{X} \sim_{\mathbb{Q}}-\mu K_{X}$, где $\mu<1 / 2$. Тогда $\operatorname{LCS}\left(X, B_{X}\right)=L_{2}$.

ДокАЗАтельство. Пусть $P$ - любая точка множества $\operatorname{LCS}\left(X, B_{X}\right)$. Тогда $P \in L_{2}$, поскольку $\operatorname{lct}\left(\mathbb{P}^{1} \times \mathbb{P}^{1}\right)=1 / 2$ и существует бирациональный морфизм $X \rightarrow \mathbb{P}^{1} \times \mathbb{P}^{1}$, который стягивает только кривую $L_{2}$.

Предположим, что $\operatorname{LCS}\left(X, B_{X}\right) \neq L_{2}$. Тогда $\operatorname{LCS}\left(X, B_{X}\right)=P$ по теореме 2.7.

Можно считать, что $P \notin L_{3}$. Тогда существует бирациональный морфизм $\varphi: X \rightarrow \mathbb{P}^{2}$, который стягивает кривые $L_{1}$ и $L_{3}$. Пусть $C_{1}$ и $C_{3}$ - собственные прообразы на поверхности $X$ достаточно общих прямых в $\mathbb{P}^{2}$, которые проходят через точки $\varphi\left(L_{1}\right)$ и $\varphi\left(L_{3}\right)$ соответственно. Тогда $-K_{X} \sim C_{1}+2 C_{3}+L_{3}$, но $C_{1} \not \ngtr P \notin C_{3}$. Мы видим, что

$$
C_{3} \cup P \subseteq \operatorname{LCS}\left(X, \lambda D+\frac{1}{2}\left(C_{1}+2 C_{3}+L_{3}\right)\right) \subseteq C_{3} \cup P \cup L_{3},
$$

что невозможно по теореме 2.7 , поскольку $P \notin L_{3}$.

Лемма 4.10. Предположим, что $O=\operatorname{Sing}(X), K_{X}^{2}=7$ u $B_{X} \sim_{\mathbb{Q}} C+(4 / 3) L$, где $L \cong \mathbb{P}^{1} \cong C$ - кривые на поверхности $X$ такие, что $L \cdot L=-1 / 2, C \cdot C=-1$, $C \cdot L=1$, но особенности лог-пары $\left(X, B_{X}\right)$ не являются лог-каноническими в некоторой точке $P \in C$. Тогда $P \in L$.

ДокАЗАТЕЛьство. Пусть $S$ - квадратичный конус в $\mathbb{P}^{3}$. Тогда $S \cong \mathbb{P}(1,1,2)$ и существует бирациональный морфизм $\varphi: X \rightarrow S \subset \mathbb{P}^{3}$, который стягивает кривую $C$ в неособую точку $Q \in S$. Тогда $Q \in \varphi(L) \in\left|\mathscr{O}_{\mathbb{P}(1,1,2)}(1)\right|$.

Предположим, что $P \notin L$. Тогда из замечания 2.22 следует, что для завершения доказательства можно считать, что либо $C \not \subset \operatorname{Supp}\left(B_{X}\right)$, либо $L \not \subset$ $\operatorname{Supp}\left(B_{X}\right)$, так как лог-пара $(X, C+(4 / 3) L)$ имеет лог-канонические особенно- 
сти в точке $P \in X$. Предположим, что $C \not \subset \operatorname{Supp}\left(B_{X}\right)$. Тогда $1 / 3=B_{X} \times$ $C \geqslant \operatorname{mult}_{P}\left(B_{X}\right)>1$, что является противоречием. Итак, показано, что $C \subset$ $\operatorname{Supp}\left(B_{X}\right)$. Значит, мы можем считать, что $L \not \subset \operatorname{Supp}\left(B_{X}\right)$.

Положим $B_{X}=\varepsilon C+\Omega$, где $\Omega$ - эффективный $\mathbb{Q}$-дивизор такой, что $C \not \subset$ $\operatorname{Supp}(\Omega)$. Тогда $1 / 3=B_{X} \cdot L=\varepsilon+\Omega \cdot L \geqslant \varepsilon$, откуда следует, что выполнено неравенство $\varepsilon \leqslant 1 / 3$. Тогда $1<\Omega \cdot C=1 / 3+\varepsilon \leqslant 2 / 3$ по лемме 2.20 . Противоречие. Лемма доказана.

\section{5. Торические многообразия}

Основная цель данного раздела - доказать лемму 5.1 (ср. [30], [55]).

Пусть $N=\mathbb{Z}^{n}$ - решетка ранга $n$, а $M=\operatorname{Hom}(N, \mathbb{Z})$ - двойственная решетка. Положим $M_{\mathbb{R}}=M \otimes_{\mathbb{Z}} \mathbb{R}$ и $N_{\mathbb{R}}=N \otimes_{\mathbb{Z}} \mathbb{R}$. Пусть $X$ - торическое многообразие, заданное полным веером $\Sigma \subset N_{\mathbb{R}}$; обозначим через $\Delta_{1}=\left\{v_{1}, \ldots, v_{m}\right\}$ множество примитивных векторов на одномерных гранях веера $\Sigma$. Пусть

$$
\Delta=\left\{w \in M \mid\left\langle w, v_{i}\right\rangle \geqslant-1 \text { для всех } i=1, \ldots, m\right\} .
$$

Положим $T=\left(\mathbb{C}^{*}\right)^{n} \subset \operatorname{Aut}(X)$. Пусть $\mathscr{N}-$ нормализатор тора $T$ в $\operatorname{Aut}(X)$ и $\mathscr{W}=\mathscr{N} / T$.

Лемма 5.1. Пусть $G \subset \mathscr{W}$ - подгруппа. Предположим, что многообразие $X$ является $\mathbb{Q}$-бакториальным. Тогда

$$
\operatorname{lct}(X, G)=\frac{1}{1+\max \left\{\langle w, v\rangle \mid w \in \Delta^{G}, v \in \Delta_{1}\right\}},
$$

где $\Delta^{G}$ - множество точек в $\Delta$, неподвижных относительно действия группь $G$.

ДокАЗАТЕЛЬСтво. Положим $\mu=1+\max \left\{\langle w, v\rangle \mid w \in \Delta^{G}, v \in \Delta_{1}\right\}$. Тогда $\mu \in \mathbb{Q}$ - наибольшее число такое, что $-K_{X} \sim_{\mathbb{Q}} \lambda R+H$, где $R$ является $T \rtimes G$-инвариантным эффективным дивизором Вейля, а $H$ - эффективный $\mathbb{Q}$-дивизор. Следовательно, $\operatorname{lct}(X, G) \leqslant 1 / \mu$.

Предположим, что $\operatorname{lct}(X, G)<1 / \mu$. Тогда найдется $G$-инвариантный эффективный $\mathbb{Q}$-дивизор $D \sim_{\mathbb{Q}}-K_{X}$ такой, что лог-пара $(X, \lambda D)$ не лог-канонична при некотором $\lambda<1 / \mu$.

Существует семейство $\left\{D_{t} \mid t \in \mathbb{C}\right\} \quad G$-инвариантных эффективных $\mathbb{Q}$-дивизоров такое, что

- $D_{t} \sim_{\mathbb{Q}} D$ при всех $t \in \mathbb{C}$,

$-D_{1}=D$,

- для любого $t \neq 0$ существует автоморфизм $\varphi_{t} \in \operatorname{Aut}(X)$ такой, что $D_{t}=$ $\varphi_{t}(D) \cong D$,

- дивизор $D_{0}$ является $T$-инвариантным, откуда следует, что лог-пара $\left(X, \lambda D_{0}\right)$ не лог-канонична (см. [21]).

С другой стороны, никакая компонента $\operatorname{Supp}\left(D_{0}\right)$ не входит в $D_{0}$ с кратностью, большей $\mu$; следовательно, лог-пара $\left(X, \lambda D_{0}\right)$ лог-канонична (см. [56]), противоречие. 
Следствие 5.2. Пусть $X=\mathbb{P}\left(\mathscr{O}_{\mathbb{P}^{n}} \oplus \mathscr{O}_{\mathbb{P}^{n}}\left(-a_{1}\right) \oplus \cdots \oplus \mathscr{O}_{\mathbb{P}^{n}}\left(-a_{k}\right)\right)$, где $a_{i} \geqslant 0$ при всех $i=1, \ldots, k$. Тогда

$$
\operatorname{lct}(X)=\frac{1}{1+\max \left\{k, n+\sum_{i=1}^{k} a_{i}\right\}} .
$$

ДокАЗАТЕЛЬство. Заметим, что $X$ является торическим многообразием, и множество $\Delta_{1}$ состоит из следующих векторов:

$$
\begin{gathered}
(\overbrace{1,0, \ldots, 0}^{k}, \overbrace{0,0, \ldots, 0}^{n}), \ldots,(0, \ldots, 0,1,0,0, \ldots, 0), \\
(-1, \ldots,-1,0,0, \ldots, 0), \\
(0,0, \ldots, 0,1,0, \ldots, 0), \ldots,(0,0, \ldots, 0,0, \ldots, 0,1) \\
\left(-a_{1}, \ldots,-a_{k},-1, \ldots,-1\right)
\end{gathered}
$$

откуда по лемме 5.1 следует требуемое утверждение.

Применяя следствие 5.2 , получаем

СлеДСтвиЕ 5.3. В обозначениях раздела 1 выполнено равенство $\operatorname{lct}(X)=$ $1 / 4$, если $\mathrm{I}(X) \in\{2.33,2.35\}, u \operatorname{lct}(X)=1 / 5$, если $\mathrm{I}(X)=2.36$.

Несложные вычисления с применением леммы 5.1 приводят к следующему результату.

СлЕДСТВИЕ 5.4. В обозначениях раздела 1 выполнено равенство

$$
\operatorname{lct}(X)= \begin{cases}1 / 3, & \text { если } \mathrm{J}(X) \in\{3.25,3.31,4.9,4.11,5.2\} \\ 1 / 4, & \text { если } \mathrm{J}(X) \in\{3.26,3.30,4.12\} \\ 1 / 5, & \text { если } \mathrm{J}(X)=3.29\end{cases}
$$

ЗАмечАниЕ 5.5. Пусть торическое многообразие $X$ является симметрическим, т. е. $\Delta^{\mathscr{W}}=\{0\}$ (см., например, [30]). Тогда по лемме 5.1 глобальный логканонический порог $\operatorname{lct}(X, \mathscr{W})$ равен 1 . Заметим, что в работах [30] и [55] это утверждение было получено при дополнительном предположении, что многообразие $X$ неособо.

\section{6. Многообразия дель Пеццо}

На протяжении этого раздела мы будем пользоваться предположениями и обозначениями, введенными в разделе 1. Предположим, что $-K_{X} \sim 2 H$, где $H$ - дивизор Картье, неделимый в группе $\operatorname{Pic}(X)$. Цель данного раздела - доказать следующий результат.

ТЕОРема 6.1. Выполнено равенство $\operatorname{lct}(X)=1 / 2$, за исключением случая $I(X)=2.35$, когда $\operatorname{lct}(X)=1 / 4$.

Из [2; теоремы 3.1 .14 и 3.3.1] следует, что $I(X) \in\{1.11, \ldots, 1.15,2.32,2.35$, $3.27\}$. Согласно [5] и [13] (см. также лемму 2.17), равенство $\operatorname{lct}(X)=1 / 2$ выполнено при $\exists(X) \in\{1.12,1.13\}$. Из леммы 2.29 следует, что $\operatorname{lct}(X)=1 / 2$ при $\beth(X)=3.27$. По лемме 5.1 при $\beth(X)=2.35$ выполнено $\operatorname{lct}(X)=1 / 4$. 
Остается разобрать следующие случаи: $J(X) \in\{1.11,1.14,1.15,2.32\}$, причем неравенство $\operatorname{lct}(X) \leqslant 1 / 2$ очевидно, так как линейная система $|H|$ непуста.

Лемма 6.2. Пусть $\rightrightarrows(X)=2.32$. Тогда $\operatorname{lct}(X)=1 / 2$.

ДокАЗАтельство. Можно считать, что $\operatorname{lct}(X)<1 / 2$. Тогда найдется эффективный $\mathbb{Q}$-дивизор $D \sim_{\mathbb{Q}} H$ такой, что лог-пара $(X, \lambda D)$ не лог-канонична при некотором $\lambda<1$.

Многообразие $X$ является дивизором бистепени $(1,1)$ на $\mathbb{P}^{2} \times \mathbb{P}^{2}$. Имеются два естественных $\mathbb{P}^{1}$-расслоения $\pi_{1}: X \rightarrow \mathbb{P}^{2}$ и $\pi_{2}: X \rightarrow \mathbb{P}^{2}$; применяя к ним теорему 2.27 , немедленно получаем противоречие.

ЗАмечАнИЕ 6.3. Пусть $\operatorname{Pic}(X)=\mathbb{Z}[H]$ и существует эффективный $\mathbb{Q}$-дивизор $D \sim_{\mathbb{Q}} H$ такой, что лог-пара $(X, \lambda D)$ не лог-канонична при некотором $\lambda<1$. Положим $D=\varepsilon S+\Omega \sim_{\mathbb{Q}} H$, где $S$ - (неприводимая) поверхность, а $\Omega$ - эффективный $\mathbb{Q}$-дивизор такой, что $\operatorname{Supp}(\Omega) \not \supset S$. Тогда $\varepsilon \leqslant 1$, так как $\operatorname{Pic}(X)=\mathbb{Z}[H]$, откуда следует, что множество $\mathbb{L} \mathbb{C} S(X, \lambda D)$ не содержит поверхностей. Более того, для любого дивизора $H \in|H|$ множество $\operatorname{LCS}(X, \lambda D+H)$ связно по теореме 2.7. Пусть $H$ - общая поверхность в линейной системе $|H|$. Из связности множества $\operatorname{LCS}(X, \lambda D+H)$ следует, что $\operatorname{LCS}(X, \lambda D+H)$ не содержит изолированных нульмерных компонент вне базисного множества линейной системы $|H|$. При этом линейная система $|H|$ не имеет базисных точек, за исключением случая $\mathrm{I}(X)=1.11$, когда базисное множество линейной системы $|H|$ состоит из единственной точки $O$. Заметим, что в последнем случае $O \notin \operatorname{LCS}(X, \lambda D)$, так как многообразие $X$ покрывается кривыми антиканонической степени 2 , проходящими через точку $O$. Следовательно, множество $\operatorname{LCS}(X, \lambda D)$ никогда не имеет изолированных нульмерных компонент и, в частности, содержит некоторую (неприводимую) кривую $C$. Положим $\left.D\right|_{H}=\bar{D}$. Тогда $-K_{H} \sim_{\mathbb{Q}} \bar{D}$ и при этом лог-пара $(H, \lambda \bar{D})$ не является лог-канонической в каждой точке пересечения $H \cap C$. Множество $\operatorname{LCS}(H, \lambda \bar{D})$ должно быть связно по теореме 2.7. Но схема $\mathscr{L}(H, \lambda \bar{D})$ нульмерна, так что $H \cdot C=|H \cap C|=1$, и множество $\operatorname{LCS}(X, \lambda D)$ не содержит других кривых, кроме $C$.

Лемма 6.4. Пусть $I(X)=1.14$. Тогда $\operatorname{lct}(X)=1 / 2$.

ДокАЗАтельство. Можно считать, что $\operatorname{lct}(X)<1 / 2$. Тогда найдется эффективный $\mathbb{Q}$-дивизор $D \sim_{\mathbb{Q}} H$ такой, что лог-пара $(X, \lambda D)$ не лог-канонична при некотором $\lambda<1$.

Линейная система $|H|$ задает вложение $X \subset \mathbb{P}^{5}$ такое, что $X$ является полным пересечением двух квадрик. Тогда $\operatorname{LCS}(X, \lambda D)$ состоит из одной прямой $C \subset X$ по замечанию 6.3 .

Из [2; предложение 3.4.1] следует, что существует коммутативная диаграмма

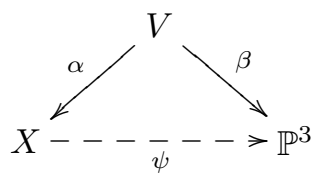

где $\psi$ - проекция из прямой $C$, морфизм $\alpha$ - раздутие прямой $C$, а $\beta$-раздутие неособой кривой $Z \subset \mathbb{P}^{3}$ степени 5 рода 2 . 
Пусть $S$ - исключительный дивизор раздутия $\beta$, а $L-$ слой морфизма $\beta$ над общей точкой кривой $Z$. Положим $\bar{S}=\alpha(S)$ и $\bar{L}=\alpha(L)$. Тогда $\bar{S} \sim 2 H$, кривая $\bar{L}$ является прямой и $\operatorname{mult}_{C}(\bar{S})=3$. При этом лог-пара $(X,(1 / 2) \bar{S})$ логканонична, т. е. можно предполагать (см. замечание 2.22$)$, что $\operatorname{Supp}(D) \not \supset \bar{S}$. Тогда $1=\bar{L} \cdot D \geqslant \operatorname{mult}_{C}(D)>1$, противоречие.

ЗАмечАниЕ 6.5. Пусть $V \subset \mathbb{P}^{5}$ - полное пересечение двух квадрик, имеющее изолированные особенности, и пусть $B_{V}-$ эффективный $\mathbb{Q}$-дивизор на $V$ такой, что $B_{V} \sim_{\mathbb{Q}}-K_{V}$ и при этом $\operatorname{LCS}\left(V, \mu B_{V}\right) \neq \varnothing$, где $\mu<1 / 2$. Рассуждая как в доказательстве леммы 6.4, получаем, что $\operatorname{LCS}\left(V, \mu B_{V}\right) \subseteq L$, где $L \subset V-$ такая прямая, что $L \cap \operatorname{Sing}(V) \neq \varnothing$.

Лемма 6.6. Пусть $\beth(X)=1.15$. Тогда $\operatorname{lct}(X)=1 / 2$.

ДокАЗАТЕльСтво. Аналогично доказательству леммы 6.4.

Лемма 6.7. Пусть $\beth(X)=1.11$. Тогда $\operatorname{lct}(X)=1 / 2$.

ДоказАтельство. Можно считать, что $\operatorname{lct}(X)<1 / 2$. Тогда найдется эффективный $\mathbb{Q}$-дивизор $D \sim_{\mathbb{Q}} H$ такой, что лог-пара $(X, \lambda D)$ не лог-канонична при некотором $\lambda<1$.

Напомним, что многообразие $X$ может быть задано уравнением

$$
\begin{aligned}
w^{2}=t^{3}+t^{2} f_{2}(x, y, z)+t f_{4}(x, y, z)+f_{6}(x, y, z) & \subset \mathbb{P}(1,1,1,2,3) \\
& \cong \operatorname{Proj}(\mathbb{C}[x, y, z, t, w]),
\end{aligned}
$$

где $\operatorname{wt}(x)=\operatorname{wt}(y)=\operatorname{wt}(z)=1, \operatorname{wt}(t)=2, \operatorname{wt}(w)=3$, a $f_{i}-$ многочлен степени $i$.

По замечанию 6.3 множество $\operatorname{LCS}(X, \lambda D)$ состоит из кривой $C \subset X$ такой, что $H \cdot C=1$.

Пусть $\psi: X \rightarrow \mathbb{P}^{2}$ - естественная проекция. Тогда $\psi$ не определена в точке $O$, заданной уравнениями $x=y=z=0$. Кривая $C$ не проходит через точку $O$, так как иначе

$$
1=\Gamma \cdot D \geqslant \operatorname{mult}_{O}(D) \operatorname{mult}_{O}(\Gamma) \geqslant \operatorname{mult}_{C}(D)>1 / \lambda>1,
$$

где $\Gamma$ - общий слой проекции $\psi$. Таким образом, $\psi(C) \subset \mathbb{P}^{2}-$ прямая.

Пусть $S$ - (единственная) поверхность из линейной системы $|H|$, проходящая через кривую $C$. Пусть $L$ - общий слой рационального отображения $\psi$, пересекающий кривую $C$. Тогда $L \subset \operatorname{Supp}(D)$, так как иначе $1=D \cdot L \geqslant \operatorname{mult}_{C}(D)>$ $1 / \lambda>1$.

Согласно замечанию 2.22 , можно считать, что $D=S$. Тогда $S$ имеет каспидальную особенность вдоль кривой $C$. Можно считать, что поверхность $S$ задана на $X$ уравнением $x=0$, а кривая $C$ - уравнениями $w=t=x=0$. Тогда $S$ задается как

$$
w^{2}=t^{3}+t^{2} f_{2}(0, y, z)+t f_{4}(0, y, z) \subset \mathbb{P}(1,1,2,3) \cong \operatorname{Proj}(\mathbb{C}[y, z, t, w]),
$$

причем $f_{6}(x, y, z)=x f_{5}(x, y, z)$, где $f_{5}(x, y, z)$ - однородный многочлен степени 5. 
Так как поверхность $S$ особа вдоль кривой $C$, то $f_{4}(x, y, z)=x f_{3}(x, y, z)$, где $f_{3}(x, y, z)$ - однородный многочлен степени 3 . Тогда каждая точка множества

$$
x=f_{5}(x, y, z)=t=w=0 \subset \mathbb{P}(1,1,1,2,3)
$$

должна быть особой на $X$, что невозможно, так как многообразие $X$ гладкое.

Таким образом, утверждение теоремы 6.1 полностью доказано.

\section{7. Многообразия с числом Пикара $\rho=2$}

На протяжении этого раздела мы будем пользоваться предположениями и обозначениями, введенными в разделе 1.

Лемма 7.1. Пусть $I(X)=2.1$ или $\beth(X)=2.3$. Тогда $\operatorname{lct}(X)=1 / 2$.

ДоказАтельство. Существует бирациональный морфизм $\alpha: X \rightarrow V$, стягивающий поверхность $E \subset X$ на неособую эллиптическую кривую $C \subset V$, где $V$ - одно из следующих многообразий Фано: неособая гиперповерхность степени 6 в $\mathbb{P}(1,1,1,2,3)$; неособая гиперповерхность степени 4 в $\mathbb{P}(1,1,1,1,2)$.

Кривая $C$ содержится в поверхности $H \subset V$ такой, что $\operatorname{Pic}(V)=\mathbb{Z}[H]$ и $-K_{X} \sim 2 H$. При этом $C$ является полным пересечением двух дивизоров из линейной системы $|H|$ и $-K_{X} \sim 2 \bar{H}+E$, где $E$ - исключительный дивизор бирационального морфизма $\alpha$, а $\bar{H}$ - собственный прообраз поверхности $H$ на многообразии $X$. В частности, выполнено неравенство $\operatorname{lct}(X) \leqslant 1 / 2$.

Предположим, что $\operatorname{lct}(X)<1 / 2$. Тогда найдется эффективный $\mathbb{Q}$-дивизор $D \sim_{\mathbb{Q}}-K_{X}$ такой, что лог-пара $(X, \lambda D)$ не лог-канонична при некотором $\lambda<1 / 2$. В этом случае $\operatorname{LCS}(X, \lambda D) \subseteq E$, так как $\operatorname{lct}(V)=1 / 2$ по теореме 6.1 и $\alpha(D) \sim_{\mathbb{Q}} 2 H \sim-K_{V}$.

Положим $k=H \cdot C$. Тогда $k=H^{3} \in\{1,2\}$. Заметим, что

$$
\mathscr{N}_{C / V} \cong \mathscr{O}_{C}\left(\left.H\right|_{C}\right) \oplus \mathscr{O}_{C}\left(\left.H\right|_{C}\right) \text {, }
$$

откуда следует $E \cong C \times \mathbb{P}^{1}$. Пусть $Z \cong C$ и $L \cong \mathbb{P}^{1}-$ кривые на $E$ такие, что $Z \cdot Z=L \cdot L=0$ и $Z \cdot L=1$. Тогда $\left.\alpha^{*}(H)\right|_{E} \sim k L$, и так как

$$
-\left.\left.2 Z \sim K_{E} \sim\left(K_{X}+E\right)\right|_{E} \sim\left(2 E-2 \alpha^{*}(H)\right)\right|_{E} \sim-2 k L+\left.2 E\right|_{E}
$$

то $\left.E\right|_{E} \sim-Z+k L$. Положим $D=\mu E+\Omega$, где $\Omega$ - эффективный $\mathbb{Q}$-дивизор на $X$ такой, что $E \not \subset \operatorname{Supp}(\Omega)$. Пара $(X, E+\lambda \Omega)$ не лог-канонична в окрестности $E$. Следовательно, пара $\left(E,\left.\lambda \Omega\right|_{E}\right)$ также не лог-канонична по теореме 2.19. Но

$$
\left.\left.\left.\Omega\right|_{E} \sim_{\mathbb{Q}}\left(-K_{X}-\mu E\right)\right|_{E} \sim_{\mathbb{Q}}\left(2 \alpha^{*}(H)-(1+\mu) E\right)\right|_{E} \sim_{\mathbb{Q}}(1+\mu) Z+k(1-\mu) L,
$$

и $0 \leqslant \lambda k(1-\mu) \leqslant 1$, что противоречит лемме 2.23 .

Лемма 7.2. Пусть $\Xi(X)=2.4$ и $X$ общее. Тогда $\operatorname{lct}(X)=3 / 4$.

ДокАзАтельство. Многообразие $X$ включается в коммутативную диаграмму

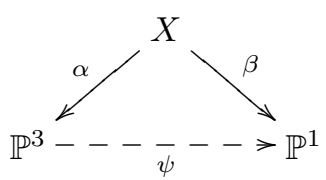


где $\psi$ - рациональное отображение, $\alpha$ - раздутие неособой кривой $C \subset \mathbb{P}^{3}$ такой, что $C=H_{1} \cdot H_{2}$ для некоторых $H_{1}, H_{2} \in\left|\mathscr{O}_{\mathbb{P}^{3}}(3)\right|$, а $\beta$ - расслоение на кубические поверхности.

Пусть $\mathscr{P}$ - пучок в $\left|\mathscr{O}_{\mathbb{P}^{3}}(3)\right|$, порожденный $H_{1}$ и $H_{2}$. Тогда $\psi$ задается пучком $\mathscr{P}$.

Предположим, что $X$ удовлетворяет следующим условиям общности: любая поверхность в $\mathscr{P}$ имеет не более одной обыкновенной двойной точки; кривая $C$ не содержит точек Эккарда ${ }^{4}$ (см. определение 3.1 ) никакой поверхности из пучка $\mathscr{P}$.

Пусть $E$ - исключительный дивизор раздутия $\alpha$. Тогда

$$
\frac{4}{3} \bar{H}_{1}+\frac{1}{3} E \sim_{\mathbb{Q}} \frac{4}{3} \bar{H}_{2}+\frac{1}{3} E \sim_{\mathbb{Q}}-K_{X},
$$

где $\bar{H}_{i}$ - собственный прообраз $H_{i}$ на многообразии $X$. В частности, выполнено неравенство $\operatorname{lct}(X) \leqslant 3 / 4$.

Предположим, что $\operatorname{lct}(X)<3 / 4$. Тогда найдется эффективный $\mathbb{Q}$-дивизор $D \sim \mathbb{Q}-K_{X}$ такой, что лог-пара $(X, \lambda D)$ не лог-канонична при некотором $\lambda<3 / 4$.

Допустим, что множество $\mathbb{L} \mathbb{C S}(X, \lambda D)$ содержит (неприводимую) поверхность $S \subset X$. Тогда $D=\varepsilon S+\Delta$, где $\varepsilon \geqslant 1 / \lambda$, а $\Delta-$ эффективный $\mathbb{Q}$-дивизор такой, что $S \not \subset \operatorname{Supp}(\Delta)$. Тогда по замечанию 2.3 лог-пара $\left(\bar{H}_{1},\left.D\right|_{\bar{H}_{1}}\right)$ не лог-канонична, если $S \cap \bar{H}_{1} \neq \varnothing$. Но $\left.D\right|_{\bar{H}_{1}} \sim_{\mathbb{Q}}-K_{\bar{H}_{1}}$. Можно считать, что $\bar{H}_{1}-$ гладкая кубическая поверхность в $\mathbb{P}^{3}$. Таким образом, по теореме $3.2 S \cap \bar{H}_{1}=\varnothing$ и, в частности, $S \sim \bar{H}_{1}$. Следовательно, $\alpha(S)$ является поверхностью из пучка $\mathscr{P}$. Тогда $\varepsilon \alpha(S)+\alpha(\Delta) \sim_{\mathbb{Q}} \mathscr{O}_{\mathbb{P} 3}(4)$, что невозможно, так как $\varepsilon \geqslant 1 / \lambda>4 / 3$.

Пусть $F$ - такой слой отображения $\beta$, что $F \cap \operatorname{LCS}(X, \lambda D) \neq \varnothing$. Положим $D=\mu F+\Omega$, где $\Omega$ - эффективный $\mathbb{Q}$-дивизор такой, что $F \not \subset \operatorname{Supp}(\Omega)$. Тогда лог-пара $\left(F,\left.\lambda \Omega\right|_{F}\right)$ не лог-канонична по теореме 2.19 , так как $\lambda \mu<1$. Из теоремы 3.2 следует, что $\operatorname{LCS}\left(F,\left.\lambda \Omega\right|_{F}\right)=O$, где $O$ - либо точка Эккарда поверхности $F$, либо ее особая точка. По теореме 2.7

$$
\operatorname{LCS}(X, \lambda D)=\operatorname{LCS}(X, \lambda \mu F+\lambda \Omega D)=O,
$$

так как по теореме 2.19 лог-пара $(X, F+\lambda \Omega D)$ не лог-канонична в точке $O$, но лог-канонична в проколотой окрестности $O$. Но $O \notin E$ по условию общности. Следовательно,

$$
\alpha(O) \subset \operatorname{LCS}\left(\mathbb{P}^{3}, \lambda \alpha(D)\right) \subseteq \alpha(O) \cup C,
$$

где $\alpha(O) \notin C$. С другой стороны, $\lambda<3 / 4$, что противоречит лемме 2.8 .

Лемма 7.3. Пусть $I(X) \in\{2.5,2.10,2.14\}$ и $X$ общее. Тогда $\operatorname{lct}(X)=1 / 2$.

ДокАзАтельство. Многообразие $X$ включается в коммутативную диаграмму

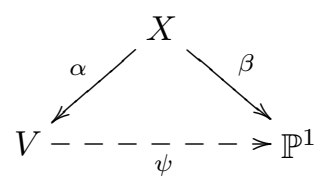

\footnotetext{
4Заметим, что кривая $C$ также не содержит особых точек поверхностей из пучка $\mathscr{P}$, так как $C$ является полным пересечением двух таких поверхностей.
} 
где $V$ - неособое многообразие Фано с $-K_{V} \sim 2 H$ для некоторого $H \in \operatorname{Pic}(V)$ и $I(V) \in\{1.13,1.14,1.15\}$, морфизм $\alpha$ является раздутием неособой кривой $C \subset V$ такой, что $C=H_{1} \cdot H_{2}$ для некоторых $H_{1}, H_{2} \in|H|, H_{1} \neq H_{2}$, морфизм $\beta$ является расслоением на поверхности дель Пеццо, а $\psi$ - проекцией из кривой $C$.

Пусть $E$ - исключительный дивизор раздутия $\alpha$. Тогда выполнены эквивалентности $2 \bar{H}_{1}+E \sim 2 \bar{H}_{2}+E \sim-K_{X}$, где $\bar{H}_{i}-$ собственный прообраз $H_{i}$ на многообразии $X$. В частности, $\operatorname{lct}(X) \leqslant 1 / 2$.

Предположим, что $\operatorname{lct}(X)<1 / 2$. Тогда найдется эффективный $\mathbb{Q}$-дивизор $D \sim_{\mathbb{Q}}-K_{X}$ такой, что особенности лог-пары $(X, \lambda D)$ не являются лог-каноническими при некотором $\lambda<1 / 2$. Тогда $\operatorname{LCS}(X, \lambda D) \subseteq E$, так как $\alpha(D) \sim_{\mathbb{Q}}-K_{V}$ и $\operatorname{lct}(V)=1 / 2$ по теореме 6.1 .

Допустим, что многообразие $X$ удовлетворяет следующему условию общности: каждый слой расслоения $\beta$ имеет не более одной обыкновенной двойной особой точки.

Пусть $F$ - такой слой отображения $\beta$, что $F \cap \operatorname{LCS}(X, \lambda D) \neq \varnothing$. Положим $D=\mu F+\Omega$, где $\Omega$ - эффективный $\mathbb{Q}$-дивизор на $X$ такой, что $F \not \subset \operatorname{Supp}(\Omega)$. Тогда

$$
\alpha(D)=\mu \alpha(F)+\alpha(\Omega) \sim_{\mathbb{Q}} 2 \alpha(F) \sim_{\mathbb{Q}}-K_{V},
$$

откуда следует $\mu \leqslant 2$. Заметим, что пара $\left(F,\left.\lambda \Omega\right|_{F}\right)$ не лог-канонична по теореме 2.19. Но выполнено соотношение $\left.\Omega\right|_{F} \sim_{\mathbb{Q}}-K_{F}$, откуда $\operatorname{lct}(F) \leqslant \lambda<1 / 2$. С другой стороны, $F$ имеет не более одной обыкновенной двойной особенности и $K_{F}^{2}=H^{3} \leqslant 5$, откуда следует неравенство $\operatorname{lct}(F) \geqslant 1 / 2$ (см. примеры $1.10,1.11,4.3$ и 4.4), противоречие.

Лемма 7.4. Пусть $I(X)=2.8$ и $X$ общее. Тогда $\operatorname{lct}(X)=1 / 2$.

ДокАЗАтельство. Пусть $O \in \mathbb{P}^{3}$ и $\alpha: V_{7} \rightarrow \mathbb{P}^{3}$ - раздутие точки $O$. Тогда $V_{7} \cong \mathbb{P}\left(\mathscr{O}_{\mathbb{P}^{2}} \oplus \mathscr{O}_{\mathbb{P}^{2}}(1)\right)$ и существует $\mathbb{P}^{1}$-расслоение $\pi: V_{7} \rightarrow \mathbb{P}^{2}$. Пусть $E-$ исключительный дивизор бирационального морфизма $\alpha$. Тогда $E$ является сечением расслоения $\pi$.

Существует двумерная квартика $R \subset \mathbb{P}^{3}$ такая, что $\operatorname{Sing}(R)=O$, точка $O$ является обыкновенной двойной точкой поверхности $R$ и существует коммутативная диаграмма

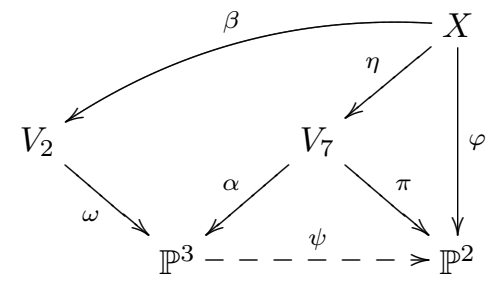

где $\omega$ - двойное накрытие, разветвленное в $R$, морфизм $\eta$ - двойное накрытие, разветвленное в собственном прообразе $R$, морфизм $\beta$ бирационален и стягивает поверхность $\bar{E}$ такую, что $\eta(\bar{E})=E$, в особую точку многообразия $V_{2}$, $\omega\left(\operatorname{Sing}\left(V_{2}\right)\right)=O$, отображение $\psi$ - проекция из точки $O$, а $\varphi$ - расслоение на коники.

Предположим, что $X$ удовлетворяет следующему условию общности: точка $O$ является обыкновенной двойной особой точкой поверхности $R$. Тогда $\bar{E} \cong \mathbb{P}^{1} \times \mathbb{P}^{1}$. 
Пусть $\bar{H}$ - собственный прообраз на $X$ общей плоскости в $\mathbb{P}^{3}$, проходящей через $O$. Тогда выполнено соотношение $-K_{X} \sim 2 \bar{H}+\bar{E}$, откуда $\operatorname{lct}(X) \leqslant 1 / 2$.

Предположим, что $\operatorname{lct}(X)<1 / 2$. Тогда найдется эффективный $\mathbb{Q}$-дивизор $D \sim_{\mathbb{Q}}-K_{X}$ такой, что лог-пара $(X, \lambda D)$ не является лог-канонической при некотором $\lambda<1 / 2$.

Из леммы 2.17 следует, что $\operatorname{LCS}(X, D) \cap \bar{E} \neq \varnothing$. Положим $D=\mu \bar{E}+\Omega$, где $\Omega$ - эффективный $\mathbb{Q}$-дивизор на $X$ такой, что $\bar{E} \not \subset \operatorname{Supp}(\Omega)$. Тогда

$$
2=D \cdot \Gamma=(\mu \bar{E}+\Omega) \cdot \Gamma=2 \mu+\Omega \cdot \Gamma \geqslant 2 \mu,
$$

где $\Gamma$ - общий слой расслоения $\varphi$. Следовательно, лог-пара $\left(\bar{E},\left.\lambda \Omega\right|_{\bar{E}}\right)$ не логканонична по теореме 2.19 , так как $\operatorname{LCS}(X, D) \cap \bar{E} \neq \varnothing$. При этом выполнена эквивалентность $\left.\Omega\right|_{\bar{E}} \sim_{\mathbb{Q}}-((1+\mu) / 2) K_{\bar{E}}$, что невозможно по лемме 2.23 .

Лемма 7.5. Пусть $I(X)=2.11$ и $X$ общее. Тогда $\operatorname{lct}(X)=1 / 2$.

ДокАЗАтельство. Пусть $V-$ кубическая гиперповерхность в $\mathbb{P}^{4}$. Тогда многообразие $X$ включается в коммутативную диаграмму

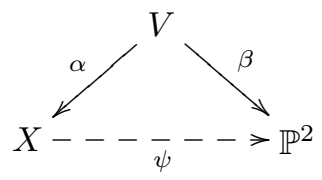

где морфизм $\alpha$ - стягивание поверхности $E \subset X$ на прямую $L \subset V$, отображение $\psi$ - проекция из прямой $L$, а морфизм $\beta$ - расслоение на коники.

Предположим, что многообразие $X$ удовлетворяет следующему условию общности: нормальное расслоение $\mathscr{N}_{L / V}$ к прямой $L$ на $V$ изоморфно $\mathscr{O}_{L} \oplus \mathscr{O}_{L}$.

Пусть $H$ - гиперплоское сечение кубики $V$ такое, что $L \subset H$. Тогда выполнена эквивалентность $-K_{X} \sim 2 \bar{H}+E$, где $\bar{H} \subset X-$ собственный прообраз поверхности $H$. В частности, $\operatorname{lct}(X) \leqslant 1 / 2$.

Предположим, что $\operatorname{lct}(X)<1 / 2$. Тогда найдется эффективный $\mathbb{Q}$-дивизор $D \sim_{\mathbb{Q}}-K_{X}$ такой, что особенности лог-пары $(X, \lambda D)$ не являются лог-каноническими при некотором $\lambda<1 / 2$. Тогда $\operatorname{LCS}(X, \lambda D) \subseteq E$, так как $\operatorname{lct}(V)=1 / 2$ и $\alpha(D) \sim_{\mathbb{Q}}-K_{V}$. Заметим, что $E \cong \mathbb{P}^{1} \times \mathbb{P}^{1}$ по условию общности.

Пусть $F \subset E$ - слой естественной проекции $E \rightarrow L$, а $Z \subset E$ - сечение этой проекции с самопересечением $Z \cdot Z=0$. Тогда $\left.\alpha^{*}(H)\right|_{E} \sim F$ и $\left.E\right|_{E} \sim-Z$, так как

$$
-2 Z-\left.\left.2 F \sim K_{E} \sim\left(K_{X}+E\right)\right|_{E} \sim 2\left(E-\alpha^{*}(H)\right)\right|_{E} \sim-2 F+\left.2 E\right|_{E} .
$$

Положим $D=\mu E+\Omega$, где $\Omega$ - эффективный $\mathbb{Q}$-дивизор на $X$ и $E \not \subset \operatorname{Supp}(\Omega)$. Тогда

$$
2=D \cdot \Gamma=\mu E \cdot \Gamma+\Omega \cdot \Gamma \geqslant \mu E \cdot \Gamma=2 \mu,
$$

где $\Gamma$ - общий слой расслоения на коники $\beta$. Таким образом, $\mu \leqslant 1$. Лог-пара $\left(E,\left.\lambda \Omega\right|_{E}\right)$ не лог-канонична по теореме 2.19. Но

$$
\left.\left.\Omega\right|_{E} \sim_{\mathbb{Q}}\left(-K_{X}-\mu E\right)\right|_{E} \sim_{\mathbb{Q}}(1+\mu) Z+2 F,
$$

что противоречит лемме 2.23 , так как $\mu \leqslant 1$ и $\lambda<1 / 2$. 
Лемма 7.6. Пусть $I(X)=2.15$ и $X$ общее. Тогда $\operatorname{lct}(X)=1 / 2$.

ДокАзАтельство. Существует бирациональный морфизм $\alpha: X \rightarrow \mathbb{P}^{3}$, который стягивает поверхность $E \subset X$ на неособую кривую $C \subset \mathbb{P}^{3}$, являющуюся полным пересечением (неприводимой, но, возможно, особой) квадрики $Q \subset \mathbb{P}^{3}$ и кубики $F \subset \mathbb{P}^{3}$.

Предположим, что многообразие $X$ удовлетворяет следующему условию общности: квадрика $Q$ неособа.

Пусть $\bar{Q}$ - собственный прообраз $Q$ на многообразии $X$. Многообразие $X$ включается в коммутативную диаграмму

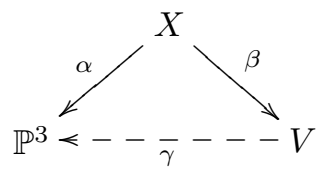

где $V$ - кубика в $\mathbb{P}^{4}$ с одной обыкновенной двойной особенностью $P \in V$, морфизм $\beta$ стягивает поверхность $\bar{Q}$ в точку $P$, а $\gamma$ является проекцией из точки $P$.

Пусть $E$ - исключительный дивизор бирационального морфизма $\alpha$. Тогда $-K_{X} \sim 2 \bar{Q}+E$ и поверхность $\beta(E) \subset V$ содержит все прямые на $V$, проходящие через $P$. В частности, $\operatorname{lct}(X) \leqslant 1 / 2$.

Предположим, что $\operatorname{lct}(X)<1 / 2$. Тогда найдется эффективный $\mathbb{Q}$-дивизор $D \sim_{\mathbb{Q}}-K_{X}$ такой, что лог-пара $(X, \lambda D)$ не является лог-канонической при некотором $\lambda<1 / 2$.

Из леммы 2.16 следует, что либо $\operatorname{LCS}(X, \lambda D) \subseteq \bar{Q}$, либо множество $\operatorname{LCS}(X$, $\lambda D)$ содержит слой естественной проекции $E \rightarrow C$. В обоих случаях мы видим, что $\operatorname{LCS}(X, \lambda D) \cap \bar{Q} \neq \varnothing$.

По условию общности $\bar{Q} \cong \mathbb{P}^{1} \times \mathbb{P}^{1}$. Положим $D=\mu \bar{Q}+\Omega$, где $\Omega$ - эффективный $\mathbb{Q}$-дивизор на $X$ такой, что $\bar{Q} \not \subset \operatorname{Supp}(\Omega)$. Тогда $\alpha(D) \sim_{\mathbb{Q}} \mu Q+\alpha(\Omega) \sim_{\mathbb{Q}}$ $-K_{\mathbb{P}^{3}}$, откуда $\mu \leqslant 2$. Лог-пара $\left(\bar{Q},\left.\lambda \Omega\right|_{\bar{Q}}\right)$ не лог-канонична по теореме 2.19 . Но $\left.\Omega\right|_{\bar{Q}} \sim_{\mathbb{Q}}-((1+\mu) / 2) K_{\bar{Q}}$, откуда по лемме 2.23 следует неравенство $\mu>1$.

По замечанию 2.22 можно предполагать, что $E \not \subset \operatorname{Supp}(D)$. Тогда

$$
1=D \cdot F=\mu \bar{Q} \cdot F+\Omega \cdot F=\mu+\Omega \cdot F \geqslant \mu,
$$

где $F$ - общий слой естественной проекции $E \rightarrow C$. Но $\mu>1$, противоречие.

Лемма 7.7. Пусть $\beth(X)=2.18$. Тогда $\operatorname{lct}(X)=1 / 2$.

ДокАзАтельство. Существует неособый дивизор $B \subset \mathbb{P}^{1} \times \mathbb{P}^{2}$ бистепени $(2,2)$ такой, что многообразие $X$ включается в коммутативную диаграмму

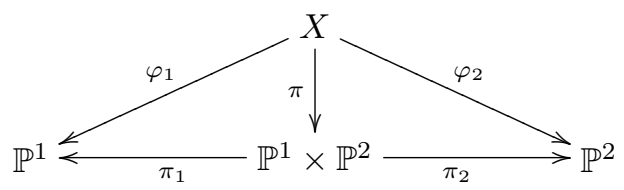

где $\pi$ - двойное накрытие, разветвленное в $B$, морфизмы $\pi_{1}$ и $\pi_{2}$ - естественные проекции, $\varphi_{1}-$ расслоение на квадрики, а $\varphi_{2}$ - расслоение на коники. 
Пусть $H_{1}$ - общий слой $\pi_{1}$, а $H_{2}$ - общая поверхность в линейной системе $\left|\pi_{2}^{*}\left(\mathscr{O}_{\mathbb{P}^{2}}(1)\right)\right|$. Тогда $B \sim 2 H_{1}+2 H_{2}$.

Пусть $\bar{H}_{1}$ - общий слой $\varphi_{1}$, а $\bar{H}_{2}$ - общая поверхность в линейной системе $\left|\varphi_{2}^{*}\left(\mathscr{O}_{\mathbb{P}^{2}}(1)\right)\right|$. Тогда $-K_{X} \sim \bar{H}_{1}+2 \bar{H}_{2}$, откуда следует неравенство $\operatorname{lct}(X) \leqslant 1 / 2$.

Предположим, что $\operatorname{lct}(X)<1 / 2$. Тогда найдется эффективный $\mathbb{Q}$-дивизор $D \sim_{\mathbb{Q}}-K_{X}$ такой, что лог-пара $(X, \lambda D)$ не является лог-канонической при некотором $\lambda<1 / 2$.

Применяя лемму 2.25 к расслоению $\varphi_{1}$, получаем $\operatorname{LCS}(X, \lambda D) \subseteq Q$, где $Q$ особый слой $\varphi_{1}$. Далее, применяя теорему $2.27 \mathrm{k}$ расслоению $\varphi_{2}$, получаем, что $\operatorname{LCS}(X, \lambda D) \subseteq Q \cap R$, где $R \subset X$ - неприводимая поверхность, заметаемая особыми слоями $\varphi_{2}$. В частности, множество $\mathbb{L} \mathbb{C} S(X, \lambda D)$ не содержит поверхностей.

Допустим, что множество $\operatorname{LCS}(X, \lambda D)$ нульмерно. Тогда

$$
\operatorname{LCS}\left(X, \lambda D+\frac{1}{2}\left(\bar{H}_{1}+2 \bar{H}_{2}\right)\right)=\operatorname{LCS}(X, \lambda D) \cup \bar{H}_{2},
$$

что невозможно по теореме 2.7 .

Значит, множество $\mathbb{L} \mathbb{C S}(X, \lambda D)$ содержит кривую $\Gamma \subset Q \cap R$. Положим $D=\mu Q+\Omega$, где $\Omega$ - эффективный $\mathbb{Q}$-дивизор такой, что $Q \not \subset \operatorname{Supp}(\Omega)$. Тогда лог-пара $\left(Q,\left.\lambda \Omega\right|_{Q}\right)$ не лог-канонична вдоль $\Gamma$ по теореме 2.19. Но $\left.\Omega\right|_{Q} \sim_{\mathbb{Q}}-K_{Q}$, откуда следует (см. лемму 4.7), что $\Gamma$ - образующая конуса $Q \subset \mathbb{P}^{3}$. Тогда $\varphi_{2}(\Gamma) \subset \mathbb{P}^{2}-$ прямая и $\varphi_{2}(\Gamma) \subseteq \varphi_{2}(R)$. Но $\varphi_{2}(R) \subset \mathbb{P}^{2}-$ плоская квартика. Таким образом, $\varphi_{2}(R)=\varphi_{2}(\Gamma) \cup Z$, где $Z \subset \mathbb{P}^{2}-$ приведенная плоская кубика. Тогда $\varphi_{2}$ индуцирует двойное накрытие кривой $\varphi_{2}(\Gamma) \backslash\left(\varphi_{2}(\Gamma) \cap Z\right)$, которое должно быть неразветвленным (см. [57]). Но особенности кривой $\varphi_{2}(R)$ не более чем нодальные (см. [57]). Поэтому $\left|\varphi_{2}(\Gamma) \cap Z\right|=3$, что невозможно, так $\operatorname{\kappa aK~} \varphi_{2}(\Gamma) \cong \mathbb{P}^{1}$.

Лемма 7.8. Пусть $\beth(X)=2.19$ и $X$ общее. Тогда $\operatorname{lct}(X)=1 / 2$.

ДокАЗАТЕЛЬство. Из [2; предложение 3.4.1] следует, что многообразие $X$ включается в коммутативную диаграмму

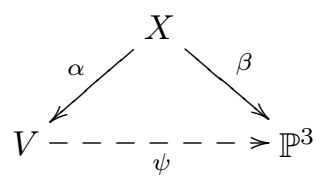

где $V$ - полное пересечение двух квадрик в $\mathbb{P}^{5}$, морфизм $\alpha$ - раздутие прямой $L \subset V$, морфизм $\beta$ - раздутие неособой кривой $C \subset \mathbb{P}^{3}$ степени 5 рода 2 , а отображение $\psi$ - проекция из прямой $L$.

Пусть $E$ и $R$ - исключительные дивизоры раздутий $\alpha$ и $\beta$ соответственно. Тогда поверхность $\beta(E) \subset \mathbb{P}^{3}$ является неприводимой квадрикой, поверхность $\alpha(R) \subset V$ заметается прямыми, лежащими на $V$ и пересекающими прямую $L$.

Предположим, что многообразие $X$ удовлетворяет следующему условию общности: поверхность $\beta(E)$ неособа.

Пусть $H$ - гиперплоское сечение $V \subset \mathbb{P}^{5}$ такое, что $L \subset H$. Тогда выполнены соотношения $2 \bar{H}+E \sim R+2 E \sim-K_{X}$, где $\bar{H}-$ собственный прообраз $H$ на многообразии $X$. В частности, $\operatorname{lct}(X) \leqslant 1 / 2$. 
Предположим, что $\operatorname{lct}(X)<1 / 2$. Тогда найдется эффективный $\mathbb{Q}$-дивизор $D \sim_{\mathbb{Q}}-K_{X}$ такой, что особенности лог-пары $(X, \lambda D)$ не являются лог-каноническими при некотором $\lambda<1 / 2$. Заметим, что $\operatorname{LCS}(X, \lambda D) \subseteq E \cong \mathbb{P}^{1} \times \mathbb{P}^{1}$, так как $\alpha(D) \sim_{\mathbb{Q}}-K_{V}$ и $\operatorname{lct}(V)=1 / 2$ по теореме 6.1 .

Пусть $F$ - слой проекции $E \rightarrow L$, а $Z$ - сечение этой проекции с самопересечением $Z \cdot Z=0$. Тогда $\left.\alpha^{*}(H)\right|_{E} \sim F$ и $\left.E\right|_{E} \sim-Z$, так как

$$
-2 Z-\left.\left.\left.2 F \sim K_{E} \sim\left(K_{X}+E\right)\right|_{E} \sim 2\left(E-\alpha^{*}(H)\right)\right|_{E} \sim 2 E\right|_{E}-2 F .
$$

Согласно замечанию 2.22 , можно считать, что либо $E \not \subset \operatorname{Supp}(D)$, либо $R \not \subset$ $\operatorname{Supp}(D)$, так как лог-пара $(X, \lambda(R+2 E))$ лог-канонична и $-K_{X} \sim R+2 E$. Положим $D=\mu E+\Omega$, где $\Omega$ - эффективный $\mathbb{Q}$-дивизор на $X$ такой, что $E \not \subset \operatorname{Supp}(\Omega)$.

Допустим, что $\mu \leqslant 1$. Тогда особенности лог-пары $(X, E+\lambda \Omega)$ не являются лог-каноническими. Значит, лог-пара $\left(E,\left.\lambda \Omega\right|_{E}\right)$ также не лог-канонична по теореме 2.19. Но

$$
\left.\left.\Omega\right|_{E} \sim_{\mathbb{Q}}\left(-K_{X}-\mu E\right)\right|_{E} \sim_{\mathbb{Q}}(1+\mu) Z+2 F,
$$

что противоречит лемме 2.23 , поскольку $\mu \leqslant 1$ и $\lambda<1 / 2$.

Таким образом, $\mu>1$. Поэтому можно считать, что $R \not \subset \operatorname{Supp}(D)$.

Пусть $\Gamma$ - общий слой проекции $R \rightarrow C$. Тогда $\Gamma \not \subset \operatorname{Supp}(D)$ и

$$
1=-K_{X} \cdot \Gamma=\mu E \cdot \Gamma+\Omega \cdot \Gamma=\mu+\Omega \cdot \Gamma \geqslant \mu,
$$

противоречие.

Лемма 7.9. Пусть $\beth(X)=2.23$ и $X$ общее. Тогда $\operatorname{lct}(X)=1 / 3$.

ДокАзАтельство. Существует бирациональный морфизм $\alpha: X \rightarrow Q$ на неособую квадрику $Q \subset \mathbb{P}^{4}$, стягивающий поверхность $E \subset X$ на неособую кривую $C \subset Q$, являющуюся полным пересечением гиперплоского сечения $H \subset Q$ и дивизора $F \in\left|\mathscr{O}_{Q}(2)\right|$.

Предположим, что многообразие $X$ удовлетворяет следующему условию общности: двумерная квадрика $H$ неособа.

Пусть $\bar{H}$ - собственный прообраз $H$ на $X$. Существует коммутативная диаграмма

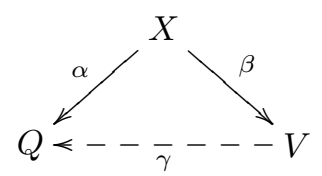

где $V$ - полное пересечение двух квадрик в $\mathbb{P}^{5}$ с одной обыкновенной двойной особенностью $P \in V$, морфизм $\beta$ - стягивание $\bar{H}$ в точку $P$, а $\gamma-$ проекция из точки $P$.

Пусть $E$ - исключительный дивизор морфизма $\alpha$. Тогда $-K_{X} \sim 3 \bar{H}+2 E$ и поверхность $\beta(E) \subset V$ содержит все прямые, лежащие на $V$ и проходящие через $P$. Имеем $\operatorname{lct}(X) \leqslant 1 / 3$.

Предположим, что $\operatorname{lct}(X)<1 / 3$. Тогда найдется эффективный $\mathbb{Q}$-дивизор $D \sim_{\mathbb{Q}}-K_{X}$ такой, что лог-пара $(X, \lambda D)$ не является лог-канонической при некотором $\lambda<1 / 3$. 
Из замечания 6.5 следует, что либо $\operatorname{LCS}(X, \lambda D) \subseteq \bar{H}$, либо множество $\operatorname{LCS}(X, \lambda D)$ содержит слой естественной проекции $E \rightarrow C$. В обоих случаях $\operatorname{LCS}(X, \lambda D) \cap \bar{H} \neq \varnothing$.

По условию общности $\bar{H} \cong \mathbb{P}^{1} \times \mathbb{P}^{1}$. Положим $D=\mu \bar{H}+\Omega$, где $\Omega$ - эффективный $\mathbb{Q}$-дивизор на $X$ такой, что $\bar{H} \not \subset \operatorname{Supp}(\Omega)$. Тогда $\alpha(D) \sim_{\mathbb{Q}} \mu H+\alpha(\Omega) \sim_{\mathbb{Q}}$ $-K_{Q}$, откуда $\mu \leqslant 3$. Лог-пара $\left(\bar{H},\left.\lambda \Omega\right|_{\bar{H}}\right)$ не лог-канонична по теореме 2.19 . Но $\left.\Omega\right|_{\bar{H}} \sim_{\mathbb{Q}}-((1+\mu) / 2) K_{\bar{H}}$, откуда $\mu>1$ по лемме 2.23. По замечанию 2.22 можно считать, что $E \not \subset \operatorname{Supp}(D)$, так как особенности лог-пары $(X, \lambda(3 \bar{H}+2 E))$ являются лог-каноническими. Пусть $F$ - общий слой естественной проекции $E \rightarrow C$. Тогда

$$
1=D \cdot F=\mu \bar{H} \cdot F+\Omega \cdot F=\mu+\Omega \cdot F \geqslant \mu
$$

что невозможно, так как $\mu>1$.

Лемма 7.10. Пусть $I(X)=2.24$ и $X$ общее. Тогда $\operatorname{lct}(X)=1 / 2$.

ДокАзАтельство. Многообразие $X$ является дивизором бистепени $(1,2)$ в $\mathbb{P}^{2} \times \mathbb{P}^{2}$. Пусть $H_{i}$ - поверхность в линейной системе $\left|\pi_{i}^{*}\left(\mathscr{O}_{\mathbb{P}^{2}}(1)\right)\right|$, где отображение $\pi_{i}: X \rightarrow \mathbb{P}^{2}$ - проекция $X$ на $i$-й сомножитель $\mathbb{P}^{2} \times \mathbb{P}^{2}, i \in\{1,2\}$. Тогда выполнено соотношение $-K_{X} \sim 2 H_{1}+H_{2}$, откуда следует неравенство $\operatorname{lct}(X) \leqslant 1 / 2$. Заметим, что $\pi_{1}$ является расслоением на коники, а $\pi_{2}$ является $\mathbb{P}^{1}$-расслоением. Пусть $\Delta \subset \mathbb{P}^{2}$ - кривая вырождения расслоения на коники $\pi_{1}$. Тогда $\Delta$ является плоской кубикой.

Предположим, что многообразие $X$ удовлетворяет следующему условию общности: кривая $\Delta$ неприводима.

Допустим, что $\operatorname{lct}(X)<1 / 2$. Тогда найдется эффективный $\mathbb{Q}$-дивизор $D \sim_{\mathbb{Q}}$ $-K_{X}$ такой, что лог-пара $(X, \lambda D)$ не лог-канонична при некотором $\lambda<1 / 2$.

Предположим, что множество $\mathbb{L} \mathbb{C} S(X, \lambda D)$ содержит поверхность $S \subset X$. Положим $D=\mu S+\Omega$, где $\Omega$ - эффективный $\mathbb{Q}$-дивизор такой, что $S \not \subset \operatorname{Supp}(\Omega)$, и $\mu>1 / \lambda$. Пусть $F_{i}$ - общий слой расслоения $\pi_{i}, i \in\{1,2\}$. Тогда

$$
2=D \cdot F_{i}=\mu S \cdot F_{i}+\Omega \cdot F_{i} \geqslant \mu S \cdot F_{i},
$$

но при этом либо $S \cdot F_{1} \geqslant 1$, либо $S \cdot F_{2} \geqslant 1$. Таким образом, $\mu \leqslant 2$, противоречие.

По теореме 2.27 и теореме 2.7 существует слой $\Gamma_{2} \mathbb{P}^{1}$-расслоения такой, что $\operatorname{LCS}(X, \lambda D)=\Gamma_{2}$, так как множество $\mathbb{L} \mathbb{C} S(X, \lambda D)$ не содержит поверхностей.

Применяя теорему $2.27 \mathrm{k}$ расслоению на коники $\pi_{1}$, получаем $\pi_{1}\left(\Gamma_{2}\right) \subset \Delta$, что невозможно, так как $\Delta \subset \mathbb{P}^{2}$ - неприводимая кубическая кривая, а $\pi_{1}\left(\Gamma_{2}\right) \subset \mathbb{P}^{2}-$ прямая.

Лемма 7.11. Пусть $I(X)=2.25$. Тогда $\operatorname{lct}(X)=1 / 2$.

ДокАЗАтельство. Напомним, что $X$ может быть получено как раздутие $\alpha: X \rightarrow \mathbb{P}^{3}$ проективного пространства в нормальной эллиптической кривой $C$ степени 4.

Пусть $Q \subset \mathbb{P}^{3}$ - общая квадрика, содержащая $C$, а $\bar{Q} \subset X$ - собственный прообраз квадрики $Q$. Тогда $-K_{X} \sim 2 \bar{Q}+E$, где $E-$ исключительный дивизор раздутия $\alpha$. В частности, $\operatorname{lct}(X) \leqslant 1 / 2$. 
Предположим, что $\operatorname{lct}(X)<1 / 2$. Тогда найдется эффективный $\mathbb{Q}$-дивизор $D \sim_{\mathbb{Q}}-K_{X}$ такой, что лог-пара $(X, \lambda D)$ не является лог-канонической при некотором $\lambda<1 / 2$.

Заметим, что линейная система $|\bar{Q}|$ определяет расслоение на квадрики $\varphi$ : $X \rightarrow \mathbb{P}^{1}$ с неприводимыми слоями, причем по теореме 2.27 лог-пара $(X, \lambda D)$ логканонична вдоль любого неособого слоя $\widetilde{Q}$ расслоения $\varphi$, поскольку выполнено неравенство $\operatorname{lct}(\widetilde{Q})=1 / 2$ (см. пример 1.10).

Множество $\operatorname{LCS}(X, \lambda D)$ не содержит слоев расслоения $\varphi$, так как $\alpha(D) \sim_{\mathbb{Q}}$ $2 Q$ и все слои $\varphi$ неприводимы. Таким образом, $\operatorname{dim}(\operatorname{LCS}(X, \lambda D)) \leqslant 1$.

Пусть $Z \in \mathbb{L} \mathbb{C S}(X, \lambda D)$. Тогда для некоторого особого слоя $\bar{Q}_{1}$ расслоения $\varphi$ выполнено $Z \subset \bar{Q}_{1}$. Заметим, что у $\varphi$ всего четыре особых слоя и каждый из них является собственным прообразом квадратичного конуса в $\mathbb{P}^{3}$ с вершиной, не лежащей на кривой $C$.

Пусть $\bar{Q}_{2}$ - особый слой расслоения $\varphi$, отличный от $\bar{Q}_{1} ;$ пусть $\bar{H}-$ собственный прообраз общей плоскости в $\mathbb{P}^{3}$, касающейся конуса $\alpha\left(\bar{Q}_{2}\right) \subset \mathbb{P}^{3}$ вдоль какой-либо его образующей $L \subset \alpha\left(\bar{Q}_{2}\right)$; пусть $\bar{R}$ - собственный прообраз достаточно общей плоскости в $\mathbb{P}^{3}$. Положим

$$
\Delta=\lambda D+\frac{1}{2}\left((1+\varepsilon) \bar{Q}_{2}+(2-\varepsilon) \bar{H}+3 \varepsilon R\right)
$$

для некоторого положительного рационального числа $\varepsilon<1-2 \lambda$. Тогда

$$
\Delta \sim_{\mathbb{Q}}-\left(\lambda+\frac{1}{2}(1+\varepsilon)\right) K_{X} \sim_{\mathbb{Q}}-\frac{1+\varepsilon+2 \lambda}{2} K_{X},
$$

откуда следует, что дивизор $-\left(K_{X}+\Delta\right)$ обилен.

Пусть $\bar{L}$ - собственный прообраз на $X$ прямой $L$. Тогда

$$
Z \cup \bar{L} \subset \operatorname{LCS}(X, \Delta) \subset \bar{Q}_{1} \cup \bar{Q}_{2},
$$

что невозможно по теореме 2.7 , так как дивизор $-\left(K_{X}+\Delta\right)$ обилен.

Лемма 7.12. Пусть $I(X)=2.26$ и $X$ общее. Тогда $\operatorname{lct}(X)=1 / 2$.

ДокАЗАтеЛьство. Пусть $V$ - трехмерное многообразие Фано с $-K_{V} \sim 2 H$ и $\operatorname{Pic}(V)=\mathbb{Z}[H]$, где $H$ - дивизор Картье такой, что $H^{3}=5$ (т. е. $J(V)=1.15$ ). Тогда линейная система $|H|$ задает вложение $X \subset \mathbb{P}^{6}$.

Из [2; предложение 3.4.1] следует, что существует прямая $L \subset X \subset \mathbb{P}^{6}$ такая, что многообразие $X$ включается в коммутативную диаграмму

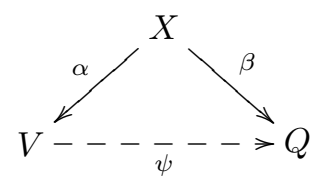

где $Q$ - неособая квадрика в $\mathbb{P}^{4}$, морфизм $\alpha$ - раздутие прямой $L \subset V$, морфизм $\beta$ - раздутие скрученной кубики $\mathbb{P}^{1} \cong C \subset Q$, а $\psi$ - проекция из прямой $L$.

Пусть $S$ - исключительный дивизор раздутия $\beta$. Положим $\bar{S}=\alpha(S)$. Тогда $\bar{S} \sim H$ и поверхность $\bar{S}$ особа вдоль прямой $L$. Пусть $E-$ исключительный дивизор раздутия $\alpha$. Тогда $\left.\beta(E) \sim \mathscr{O}_{\mathbb{P}^{4}}(1)\right|_{Q}$, откуда следует, что $\beta(E)-$ неприводимая квадрика. 
Предположим, что $X$ удовлетворяет следующему условию общности: поверхность $\beta(E)$ неособа.

Заметим, что $-K_{X} \sim 2 S+3 E$. Более того, лог-пара $(X,(1 / 3)(2 S+3 E))$ логканонична, но не лог-терминальна. Таким образом, $\operatorname{lct}(X) \leqslant 1 / 3$.

Предположим, что $\operatorname{lct}(X)<1 / 3$. Тогда найдется эффективный $\mathbb{Q}$-дивизор $D \sim_{\mathbb{Q}}-K_{X}$ такой, что особенности лог-пары $(X, \lambda D)$ не являются лог-каноническими при некотором $\lambda<1 / 3$. Тогда $\operatorname{LCS}(X, \lambda D) \subseteq E$, так как $\alpha(D) \sim_{\mathbb{Q}}-K_{V}$ и $\operatorname{lct}(V)=1 / 2$ по теореме 6.1 .

Заметим, что $E \cong \mathbb{P}^{1} \times \mathbb{P}^{1}$ по условию общности. Пусть $F-$ слой проекции $E \rightarrow L$, а $Z$ - сечение этой проекции такое, что $Z \cdot Z=0$. Тогда $\left.\alpha^{*}(H)\right|_{E} \sim F$ и $\left.E\right|_{E} \sim-Z$, так как

$$
-2 Z-\left.\left.\left.2 F \sim K_{E} \sim\left(K_{X}+E\right)\right|_{E} \sim 2\left(E-\alpha^{*}(H)\right)\right|_{E} \sim 2 E\right|_{E}-2 F .
$$

Согласно замечанию 2.22 , можно считать, что либо $E \not \subset \operatorname{Supp}(D)$, либо $S \not \subset$ $\operatorname{Supp}(D)$. Положим $D=\mu E+\Omega$, где $\Omega$ - эффективный $\mathbb{Q}$-дивизор на $X$ такой, что $E \not \subset \operatorname{Supp}(\Omega)$.

Предположим, что $\mu \leqslant 2$. Тогда лог-пара $(X, E+\lambda \Omega)$ не лог-канонична. Значит, лог-пара $\left(E,\left.\lambda \Omega\right|_{E}\right)$ также не лог-канонична по теореме 2.19. Но

$$
\left.\left.\Omega\right|_{E} \sim_{\mathbb{Q}}\left(-K_{X}-\mu E\right)\right|_{E} \sim_{\mathbb{Q}}(1+\mu) Z+2 F,
$$

что противоречит лемме 2.23 , так как $\mu \leqslant 2$ и $\lambda<1 / 3$.

Таким образом, $\mu>2$, и можно считать, что $S \not \subset \operatorname{Supp}(D)$.

Пусть $\Gamma$ - общий слой проекции $S \rightarrow C$. Тогда $\Gamma \not \subset \operatorname{Supp}(D)$ и

$$
1=-K_{X} \cdot \Gamma=\mu E \cdot \Gamma+\Omega \cdot \Gamma=\mu+\Omega \cdot \Gamma \geqslant \mu,
$$

противоречие.

ЛЕмма 7.13. Пусть $I(X)=2.27$. Тогда $\operatorname{lct}(X)=1 / 2$.

ДокАзАтельство. Существует бирациональный морфизм $\alpha: X \rightarrow \mathbb{P}^{3}$, стягивающий поверхность $E$ на скрученную кубику $C \subset \mathbb{P}^{3}$, и $X \cong \mathbb{P}(\mathscr{E})$, где $\mathscr{E}$ стабильное векторное расслоение ранга 2 на $\mathbb{P}^{2}$ с $c_{1}(\mathscr{E})=0$ и $c_{2}(\mathscr{E})=2$ такое, что последовательность

$$
0 \rightarrow \mathscr{O}_{\mathbb{P}_{2}}(-1) \oplus \mathscr{O}_{\mathbb{P}_{2}}(-1) \rightarrow \mathscr{O}_{\mathbb{P}_{2}} \oplus \mathscr{O}_{\mathbb{P}_{2}} \oplus \mathscr{O}_{\mathbb{P}_{2}} \oplus \mathscr{O}_{\mathbb{P}_{2}} \rightarrow \mathscr{E} \otimes \mathscr{O}_{\mathbb{P}_{2}}(1) \rightarrow 0
$$

точна (см. [58; приложение 1]).

Пусть $Q \subset \mathbb{P}^{3}$ - общая квадрика, содержащая $C$, а $\bar{Q} \subset X-$ собственный прообраз $Q$. Тогда $-K_{X} \sim 2 \bar{Q}+E$, где $E-\alpha$-исключительный дивизор. Значит, $\operatorname{lct}(X) \leqslant 1 / 2$.

Предположим, что $\operatorname{lct}(X)<1 / 2$. Тогда найдется эффективный $\mathbb{Q}$-дивизор $D \sim_{\mathbb{Q}}-K_{X}$ такой, что лог-пара $(X, \lambda D)$ не является лог-канонической при некотором $\lambda<1 / 2$.

Допустим, что множество $\mathbb{L} \mathbb{C} S(X, \lambda D)$ содержит поверхность $S \subset X$. Положим $D=\mu F+\Omega$, где $\mu \geqslant 1 / \lambda$, а $\Omega$ - эффективный $\mathbb{Q}$-дивизор такой, что $F \not \subset \operatorname{Supp}(\Omega)$. 
Пусть $\varphi: X \rightarrow \mathbb{P}^{2}$ - естественное $\mathbb{P}^{1}$-расслоение. Тогда

$$
2=D \cdot \Gamma=\mu F \cdot \Gamma+\Omega \cdot \Gamma=\mu F \cdot \Gamma+\Omega \cdot F \geqslant \mu F \cdot \Gamma,
$$

где $\Gamma$ - общий слой $\varphi$. Таким образом, $F$ заметается слоями расслоения $\varphi$. Тогда выполнено соотношение $\alpha(F) \sim \mathscr{O}_{\mathbb{P}^{3}}(d)$, где $d \geqslant 2$. Но $\alpha(D) \sim_{\mathbb{Q}} \mu \alpha(F)+$ $\alpha(\Omega) \sim_{\mathbb{Q}} \mathscr{O}_{\mathbb{P}^{3}}(4)$, противоречие.

Следовательно, множество $\operatorname{LCS}(X, \lambda D)$ не содержит поверхностей. Применяя теорему 2.27 к лог-паре $(X, \lambda D)$ и расслоению $\varphi$, получаем, что $L \subseteq$ $\operatorname{LCS}(X, \lambda D)$, где $L-$ слой расслоения $\varphi$. Тогда $\alpha(L)$ является секущей прямой скрученной кубики $C \subset \mathbb{P}^{3}$. Имеем

$$
\alpha(L) \subseteq \operatorname{LCS}\left(\mathbb{P}^{3}, \lambda \alpha(D)\right) \subseteq \alpha(\operatorname{LCS}(X, \lambda D)) \cup C,
$$

что невозможно по лемме 2.9 .

Лемма 7.14. Пусть $I(X)=2.28$. Тогда $\operatorname{lct}(X)=1 / 4$.

ДокАЗАтельство. Напомним, что многообразие $X$ может быть получено как раздутие $\alpha: X \rightarrow \mathbb{P}^{3}$ проективного пространства в плоской кубической кривой $C \subset \mathbb{P}^{3}$. При этом выполнено соотношение $-K_{X} \sim 4 G+3 E$, где $E-$ исключительный дивизор раздутия $\alpha$, а $G$ - собственный прообраз плоскости в $\mathbb{P}^{3}$, содержащей кривую $C$. В частности, $\operatorname{lct}(X) \leqslant 1 / 4$.

Предположим, что $\operatorname{lct}(X)<1 / 4$. Тогда найдется эффективный $\mathbb{Q}$-дивизор $D \sim_{\mathbb{Q}}-K_{X}$ такой, что особенности лог-пары $(X, \lambda D)$ не являются лог-каноническими при некотором $\lambda<1 / 4$. Следовательно, $\operatorname{LCS}(X, \lambda D) \subseteq E$, так как $\operatorname{lct}\left(\mathbb{P}^{4}\right)=1 / 4$. Вычисляя пересечения с собственным прообразом общей прямой в $\mathbb{P}^{3}$, пересекающей кривую $C$, мы видим, что множество $\operatorname{LCS}(X, \lambda D)$ не содержит дивизор $E$. Кроме того, по лемме 2.14 любая кривая $\Gamma \in \mathbb{L} \mathbb{C} S(X, \lambda D)$ должна быть слоем естественной проекции $\psi: E \rightarrow C$. Таким образом, по теореме 2.7 множество $\operatorname{LCS}(X, \lambda D)$ состоит либо из одной точки, либо из одного слоя проекции $\psi$.

Пусть $R$ - достаточно общий конус в $\mathbb{P}^{3}$ над кривой $C$, а $H$ - достаточно общая плоскость в $\mathbb{P}^{3}$, проходящая через точку $\operatorname{Sing}(R)$. Тогда

$$
\operatorname{LCS}\left(X, \lambda D+\frac{3}{4}(\bar{R}+\bar{H})\right)=\operatorname{LCS}(X, \lambda D) \cup \operatorname{Sing}(\bar{R}),
$$

где $\bar{R}$ и $\bar{H}$ - собственные прообразы $R$ и $H$ на многообразии $X$. При этом дивизор

$$
-\left(K_{X}+\lambda D+\frac{3}{4}(\bar{R}+\bar{H})\right) \sim_{\mathbb{Q}}\left(\lambda-\frac{1}{4}\right) K_{X}
$$

обилен, что противоречит теореме 2.7 .

Лемма 7.15. Пусть $I(X)=2.29$. Тогда $\operatorname{lct}(X)=1 / 3$.

ДокАзАтельство. Напомним, что многообразие $X$ может быть получено как раздутие $\alpha: X \rightarrow Q$ неособой квадрики $Q$ в конике $C \subset Q$.

Пусть $H$ - общее гиперплоское сечение квадрики $Q \subset \mathbb{P}^{4}$, содержащее кривую $C$, а $\bar{H}$ - собственный прообраз поверхности $H$ на многообразии $X$. Тогда $-K_{X} \sim 3 \bar{H}+2 E$, где $E-$ исключительный дивизор раздутия $\alpha$. В частности, выполнено неравенство $\operatorname{lct}(X) \leqslant 1 / 3$. 
Предположим, что $\operatorname{lct}(X)<1 / 3$. Тогда найдется эффективный $\mathbb{Q}$-дивизор $D \sim_{\mathbb{Q}}-K_{X}$ такой, что особенности лог-пары $(X, \lambda D)$ не являются лог-каноническими при некотором $\lambda<1 / 3$. Тогда $\operatorname{LCS}(X, \lambda D) \subseteq E$, так как $\operatorname{lct}(Q)=1 / 3$ (см. пример 1.3) и $\alpha(D) \sim_{\mathbb{Q}}-K_{Q}$.

Линейная система $|\bar{H}|$ не имеет базисных точек и определяет морфизм $\beta$ : $X \rightarrow \mathbb{P}^{1}$, общий слой которого является гладкой двумерной квадрикой. При этом лог-пара $(X, \lambda D)$ лог-канонична вдоль неособых слоев расслоения $\beta$ по теореме 2.27 (см. пример 1.10).

Из теоремы 2.7 следует, что существует особый слой $S \sim \bar{H}$ расслоения $\beta$ такой, что $\operatorname{LCS}(X, \lambda D) \subseteq E \cap S$, а $\alpha(S) \subset \mathbb{P}^{3}$ является квадратичным конусом. Положим $\Gamma=E \cap S$. Тогда $\Gamma-($ неприводимая) коника, причем лог-пара $(X, S+(2 / 3) E)$ имеет лог-канонические особенности и $3 S+2 E \sim_{\mathbb{Q}} D$. Таким образом, по замечанию 2.22 для завершения доказательства можно считать, что либо $S \not \subset \operatorname{Supp}(D)$, либо $E \not \subset \operatorname{Supp}(D)$.

Пересекая дивизор $D$ с собственным прообразом общей образующей конуса $\alpha(S) \subset \mathbb{P}^{3}$ и общим слоем проекции $E \rightarrow C$, мы видим, что $\Gamma \nsubseteq \operatorname{LCS}(X, \lambda D)$, т. е. $\operatorname{LCS}(X, \lambda D)$ состоит из единственной точки $O \in \Gamma$ по теореме 2.7 .

Пусть $R$ - общая (не проходящая через $O$ ) поверхность в $\left|\alpha^{*}(H)\right|$. Тогда

$$
\operatorname{LCS}\left(X, \lambda D+\frac{1}{2}(\bar{H}+2 R)\right)=R \cup O,
$$

что невозможно по теореме 2.7 , так как $-K_{X} \sim \bar{H}+2 R \sim_{\mathbb{Q}} D$ и $\lambda<1 / 3$.

Лемма 7.16. Пусть $I(X)=2.30$. Тогда $\operatorname{lct}(X)=1 / 4$.

ДокАзАтельство. Многообразие $X$ включается в коммутативную диаграмму

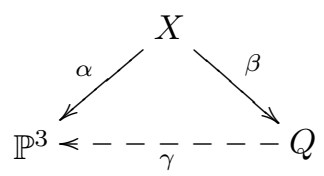

где $Q$ - неособая квадрика в $\mathbb{P}^{4}$, морфизм $\alpha$ - раздутие (неособой) коники $C \subset \mathbb{P}^{3}$, морфизм $\beta$ - раздутие точки, а отображение $\gamma-$ проекция из точки.

Пусть $G$ - собственный прообраз на многообразии $X$ единственной плоскости в $\mathbb{P}^{3}$, содержащей конику $C$. Тогда поверхность $G$ стягивается морфизмом $\beta$ и $-K_{X} \sim 4 G+3 E$, где $E-$ исключительный дивизор раздутия $\alpha$. Таким образом, $\operatorname{lct}(X) \leqslant 1 / 4$.

Предположим, что $\operatorname{lct}(X)<1 / 4$. Тогда найдется эффективный $\mathbb{Q}$-дивизор $D \sim_{\mathbb{Q}}-K_{X}$ такой, что особенности лог-пары $(X, \lambda D)$ не являются лог-каноническими при некотором $\lambda<1 / 4$. Тогда $\operatorname{LCS}(X, \lambda D) \subseteq E \cap G$, так как $\operatorname{lct}\left(\mathbb{P}^{4}\right)=1 / 4$ и $\operatorname{lct}(Q)=1 / 3$.

Согласно замечанию 2.22 , можно считать, что либо $G \not \subset \operatorname{Supp}(X)$, либо $E \not \subset$ $\operatorname{Supp}(X)$.

Пересекая $D$ с прямыми в $G \cong \mathbb{P}^{2}$ и со слоями проекции $E \rightarrow C$, мы сразу видим, что $\operatorname{LCS}(X, \lambda D) \subsetneq E \cap G$, т. е. $\operatorname{LCS}(X, \lambda D)=O$ для некоторой точки $O \in E \cap G$ по теореме 2.7 . 
Пусть $R$ - общая поверхность в линейной системе $\left|\alpha^{*}(H)\right|$, а $F$ - общая поверхность в линейной системе $\left|\alpha^{*}(2 H)-E\right|$. Тогда

$$
\operatorname{LCS}\left(X, \lambda D+\frac{1}{2}(F+2 R)\right)=R \cup O,
$$

что невозможно по теореме 2.7 , так как $-K_{X} \sim F+2 R \sim_{\mathbb{Q}} D$ и $\lambda<1 / 4$.

Лемма 7.17. Пусть $I(X)=2.31$. Тогда $\operatorname{lct}(X)=1 / 3$.

ДокАзАтельство. Напомним, что многообразие $X$ может быть получено как раздутие $\alpha: X \rightarrow Q$ неособой квадрики $Q$ в прямой $L \subset Q$.

Пусть $H$ - достаточно общее гиперплоское сечение квадрики $Q \subset \mathbb{P}^{4}$, проходящее через прямую $L$, а $\bar{H}$ - собственный прообраз поверхности $H$ на $X$. Тогда $-K_{X} \sim 3 \bar{H}+2 E$, где $E-$ исключительный дивизор раздутия $\alpha$. В частности, $\operatorname{lct}(X) \leqslant 1 / 3$.

Предположим, что $\operatorname{lct}(X)<1 / 3$. Тогда найдется эффективный $\mathbb{Q}$-дивизор $D \sim_{\mathbb{Q}}-K_{X}$ такой, что особенности лог-пары $(X, \lambda D)$ не являются лог-каноническими при некотором $\lambda<1 / 3$. Тогда $\operatorname{LCS}(X, \lambda D) \subseteq E$, так как $\operatorname{lct}(Q)=1 / 3$ и $\alpha(D) \sim_{\mathbb{Q}}-K_{Q}$.

Линейная система $|\bar{H}|$ определяет $\mathbb{P}^{1}$-расслоение $\varphi: X \rightarrow \mathbb{P}^{2}$ такое, что индуцированный морфизм $E \cong \mathbb{F}_{1} \rightarrow \mathbb{P}^{2}$ стягивает неприводимую кривую $Z \subset E$. Тогда $\operatorname{LCS}(X, \lambda D)=Z$ по теореме 2.27. Положим $D=\mu E+\Omega$, где $\Omega$ - эффективный $\mathbb{Q}$-дивизор на $X$ такой, что $E \not \subset \operatorname{Supp}(\Omega)$. Тогда

$$
2=D \cdot F=\mu E \cdot F+\Omega \cdot F=\mu+\Omega \cdot F \geqslant \mu,
$$

где $F$ - общий слой расслоения $\varphi$. Лог-пара $(X, E+\lambda \Omega)$ не лог-канонична, так как $\lambda<1 / 3$. Поэтому лог-пара $\left(E,\left.\lambda \Omega\right|_{E}\right)$ также не лог-канонична по теоремe 2.19 .

Пусть $C$ - слой естественной проекции $E \rightarrow L$. Тогда $\left.\Omega\right|_{E} \sim_{\mathbb{Q}} 3 C+(1+\mu) Z$; следовательно, лог-пара $\left(E,\left.\lambda \Omega\right|_{E}\right)$ лог-канонична по лемме 4.8, противоречие.

\section{8. Многообразия с числом Пикара $\rho=3$}

На протяжении этого раздела мы будем пользоваться предположениями и обозначениями, введенными в разделе 1.

Лемма 8.1. Пусть $I(X)=3.1$ и $X$ общее. Тогда $\operatorname{lct}(X)=3 / 4$.

ДокАзАтельство. Существует двойное накрытие $\omega: X \rightarrow \mathbb{P}^{1} \times \mathbb{P}^{1} \times \mathbb{P}^{1}$, которое разветвлено в дивизоре тристепени $(2,2,2)$. Проекция $\mathbb{P}^{1} \times \mathbb{P}^{1} \times \mathbb{P}^{1} \rightarrow \mathbb{P}^{1}$ на $i$-й сомножитель индуцирует морфизм $\pi_{i}: X \rightarrow \mathbb{P}^{1}$, слои которого являются поверхностями дель Пеццо степени 4.

Пусть $R_{1}$ - особый слой расслоения $\pi_{1}$ с особой точкой $Q ;$ пусть $R_{2}$ и $R_{3}-$ слои проекций $\pi_{2}$ и $\pi_{3}$ такие, что $R_{2} \ni Q \in R_{3}$. Тогда $\operatorname{mult}_{Q}\left(R_{1}+R_{2}+R_{3}\right)=4$, откуда следует, что лог-пара $\left(X,(3 / 4)\left(R_{1}+R_{2}+R_{3}\right)\right)$ не лог-терминальна в $Q$. При этом $-K_{X} \sim R_{1}+R_{2}+R_{3}$. Таким образом, $\operatorname{lct}(X) \leqslant 3 / 4$.

Предположим, что lct $(X)<3 / 4$. Существует эффективный $\mathbb{Q}$-дивизор $D \sim_{\mathbb{Q}}$ $-K_{X}$ такой, что лог-пара $(X, \lambda D)$ не лог-канонична в некоторой точке $P \in X$ при некотором $\lambda<3 / 4$. 
Пусть $S_{i}$ - слой отображения $\pi_{i}$, содержащий точку $P$. Так как многообразие $X$ общее, то (возможно, после перенумерации) можно считать, что

- поверхность $S_{1}$ неособа в точке $P$,

- особенности поверхности $S_{1}$ исчерпываются единственной обыкновенной двойной особенностью (если $S_{1}$ вообе особа),

- никакая неособая кривая $L \subset S_{1}$ такая, что $-K_{S_{1}} \cdot L=1$, не содержит точку $P \notin L$,

- для любых неособых кривых $C_{1} \subset S_{1} \supset C_{2}$ таких, что $-K_{S_{1}} \cdot C_{1}=$ $-K_{S_{1}} \cdot C_{2}=2$ и и $C_{1}+C_{2} \sim-K_{S_{1}}$, выполнено $P \neq C_{1} \cap C_{2}$.

Поверхность $S_{1}$ является поверхностью дель Пеццо степени 4. Положим $D=\mu S_{1}+\Omega$, где $\Omega$ - эффективный $\mathbb{Q}$-дивизор на $X$ такой, что $S_{1} \not \subset \operatorname{Supp}(\Omega)$.

Пусть $\varphi: X \rightarrow \mathbb{P}^{1} \times \mathbb{P}^{1}-$ расслоение на коники, заданное полной линейной системой $\left|S_{2}+S_{3}\right|$, и пусть $\Gamma-$ общий слой $\varphi$. Тогда

$$
2=D \cdot \Gamma=\mu S_{1} \cdot \Gamma+\Omega \cdot \Gamma=2 \mu+\Omega \cdot \Gamma \geqslant 2 \mu,
$$

откуда следует неравенство $\mu \leqslant 1$. Поэтому лог-пара $\left(X, S_{1}+\lambda \Omega\right)$ не лог-канонична в точке $P$. Следовательно, лог-пара $\left(S_{1},\left.\lambda \Omega\right|_{S_{1}}\right)$ также не лог-канонична в точке $P$ по теореме 2.19. Но $\left.\Omega\right|_{S_{1}} \sim_{\mathbb{Q}}-K_{S_{k}}$, что невозможно (ср. пример 4.4).

Лемма 8.2. Пусть $I(X)=3.2$ и $X$ общее. Тогда $\operatorname{lct}(X)=1 / 2$.

ДокАЗАтельство. Напомним, что $X$ - примитивное многообразие Фано (см. [52; определение 1.3]). Положим

$$
U=\mathbb{P}\left(\mathscr{O}_{\mathbb{P}^{1} \times \mathbb{P}^{1}} \oplus \mathscr{O}_{\mathbb{P}^{1} \times \mathbb{P}^{1}}(-1,-1) \oplus \mathscr{O}_{\mathbb{P}^{1} \times \mathbb{P}^{1}}(-1,-1)\right)
$$

пусть $\pi: U \rightarrow \mathbb{P}^{1} \times \mathbb{P}^{1}$ - естественная проекция, и пусть $L$ - тавтологическое линейное расслоение на $U$. Тогда $X \in\left|2 L+\pi^{*}\left(\mathscr{O}_{\mathbb{P}^{1} \times \mathbb{P}^{1}}(2,3)\right)\right|$.

Покажем, что $\operatorname{lct}(X) \leqslant 1 / 2$. Пусть $E_{1}$ и $E_{2}$ - такие дивизоры на многообразии $X$, что $\pi\left(E_{1}\right)$ и $\pi\left(E_{2}\right)$ являются дивизорами на $\mathbb{P}^{1} \times \mathbb{P}^{1}$ бистепеней $(1,0)$ и $(0,1)$ соответственно. Тогда выполнено соотношение $-\left.K_{X} \sim L\right|_{X}+2 E_{1}+E_{2}$, откуда следует $\operatorname{lct}(X) \leqslant 1 / 2$.

Предположим, что $\operatorname{lct}(X)<1 / 2$. Найдется эффективный $\mathbb{Q}$-дивизор $D \sim_{\mathbb{Q}}$ $-K_{X}$ такой, что лог-пара $(X, \lambda D)$ не лог-канонична в некоторой точке $P \in X$ при некотором $\lambda<1 / 2$.

Из [59; предложение 3.8$]$ следует, что $X$ включается в коммутативную диаграмму

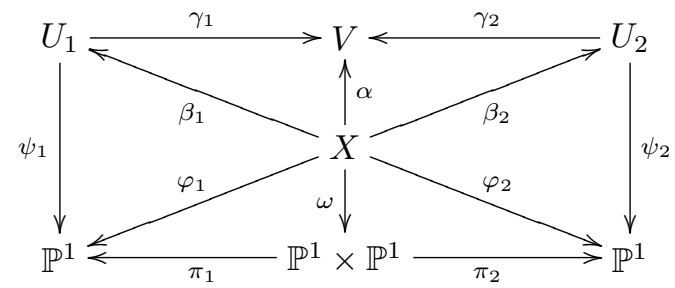

где $V$ - многообразие Фано с единственной обыкновенной двойной особенностью $O \in V$ такое, что $\operatorname{Pic}(V)=\mathbb{Z}\left[-K_{V}\right]$ и $-K_{V}^{3}=16$, морфизм $\alpha$ стягивает единственную поверхность $\mathbb{P}^{1} \times \mathbb{P}^{1} \cong S \subset X$ такую, что $\left.S \sim L\right|_{X}$, в точку $O \in V$, морфизм $\beta_{i}$ стягивает $S$ на неособую рациональную кривую, морфизм $\gamma_{i}$ 
стягивает кривую $\beta_{i}(S)$ в точку $O \in V$, причем рациональное отображение $\gamma_{2} \circ \gamma_{1}^{-1}: U_{1} \rightarrow U_{2}$ является флопом в $\beta_{1}(S) \cong \mathbb{P}^{1}$, морфизм $\psi_{2}$ является расслоением на квадрики, а морфизмы $\psi_{1}, \varphi_{1}$ и $\varphi_{2}$ являются расслоениями на поверхности дель Пеццо степеней 4,3 и 6 соответственно. Морфизмы $\pi_{1}$ и $\pi_{2}-$ естественные проекции, и $\omega=\left.\pi\right|_{X}$. Заметим, что $\mathrm{Cl}(V)=\mathbb{Z}\left[\alpha\left(E_{1}\right)\right] \oplus \mathbb{Z}\left[\alpha\left(E_{2}\right)\right]$ и $\omega$ является расслоением на коники. Кривая $\beta_{1}(S)$ является сечением расслоения $\psi_{1}$, а кривая $\beta_{2}(S)$ является 2-сечением расслоения $\psi_{2}$.

Предположим, что многообразие $X$ удовлетворяет следующему условию общности: любой слой расслоения $\varphi_{2}$ имеет особенности не хуже, чем $\mathbb{A}_{1}$.

Применяя лемму 2.25 к расслоению $\varphi_{1}$, получаем, что $\operatorname{LCS}(X, \lambda D) \subseteq S_{1}$, где $S_{1}$ - особый слой расслоения $\varphi_{1}$, так как глобальный лог-канонический порог неособой поверхности дель Пеццо степени 6 равен 1/2 (см. пример 1.10).

Применяя лемму 2.25 к расслоению $\varphi_{2}$, получаем противоречие с примером 1.11 .

Лемма 8.3. Пусть $I(X)=3.3$ и $X$ общее. Тогда $\operatorname{lct}(X)=2 / 3$.

ДокАЗАТЕЛЬство. Многообразие $X$ является дивизором тристепени $(1,1,2)$ на $\mathbb{P}^{1} \times \mathbb{P}^{1} \times \mathbb{P}^{2}$. В частности, выполнено соотношение $-K_{X} \sim \pi_{1}^{*}\left(\mathscr{O}_{\mathbb{P}}(1)\right)+$ $\pi_{2}^{*}\left(\mathscr{O}_{\mathbb{P}^{1}}(1)\right)+\varphi^{*}\left(\mathscr{O}_{\mathbb{P}^{2}}(1)\right)$, где $\pi_{1}: X \rightarrow \mathbb{P}^{1}$ и $\pi_{2}: X \rightarrow \mathbb{P}^{1}-$ расслоения на поверхности дель Пеццо степени 4 , индуцированные проекциями многообразия $\mathbb{P}^{1} \times \mathbb{P}^{1} \times \mathbb{P}^{2}$ на первый и второй множитель соответственно, а $\varphi: X \rightarrow \mathbb{P}^{2}-$ расслоение на коники, индуцированное проекцией $\mathbb{P}^{1} \times \mathbb{P}^{1} \times \mathbb{P}^{2} \rightarrow \mathbb{P}^{2}$.

Пусть $\alpha_{2}: X \rightarrow \mathbb{P}^{1} \times \mathbb{P}^{2}$ - бирациональный морфизм, заданный полной линейной системой $\left|\pi_{2}^{*}\left(\mathscr{O}_{\mathbb{P}^{1}}(1)\right)+\varphi^{*}\left(\mathscr{O}_{\mathbb{P}^{2}}(1)\right)\right|$. Пусть $H_{i} \in\left|\pi_{i}^{*}\left(\mathscr{O}_{\mathbb{P}^{1}}(1)\right)\right|$ и $R \in$ $\left|\varphi^{*}\left(\mathscr{O}_{\mathbb{P}^{2}}(1)\right)\right|$ - общие дивизоры. Тогда $H_{1} \sim H_{2}+2 R-E_{2}$, где $E_{2}-$ исключительный дивизор бирационального морфизма $\alpha_{2}$. Следовательно,

$$
-K_{X} \sim H_{1}+H_{2}+R \sim_{\mathbb{Q}} \frac{3}{2} H_{1}+\frac{1}{2} H_{2}+\frac{1}{2} E_{2},
$$

откуда вытекает $\operatorname{lct}(X) \leqslant 2 / 3$.

Предположим, что $\operatorname{lct}(X)<2 / 3$. Найдется эффективный $\mathbb{Q}$-дивизор $D \sim_{\mathbb{Q}}$ $-K_{X}$ такой, что лог-пара $(X, \lambda D)$ не является лог-канонической в некоторой точке $P \in X$ при некотором $\lambda<2 / 3$.

Пусть $S_{i}-$ слой расслоения $\pi_{i}$ такой, что $P \in S_{i}$. Так как многообразие $X$ общее, то (возможно, после перенумерации) можно считать, что

- поверхность $S_{1}$ неособа в точке $P$,

- особенности поверхности $S_{1}$ исчерпываются одной обыкновенной двойной особенностью (если $S_{1}$ вообще особа),

- для каждой неособой кривой $L \subset S_{1}$ такой, что $-K_{S_{1}} \cdot L=1$, выполнено $P \notin L$, если $\operatorname{Sing}\left(S_{1}\right) \neq \varnothing$.

Положим $D=\mu S_{1}+\Omega$, где $\Omega$ - эффективный $\mathbb{Q}$-дивизор такой, что $S_{1} \not \subset$ $\operatorname{Supp}(\Omega)$. Тогда лог-пара $\left(H_{2},\left.\lambda \mu S_{1}\right|_{H_{2}}+\left.\lambda \Omega\right|_{H_{2}}\right)$ не лог-канонична, так как $\operatorname{lct}\left(H_{2}\right)=2 / 3$. Таким образом, $\mu \leqslant 1 / \lambda$. Следовательно, лог-пара $\left(S_{1},\left.\lambda \Omega\right|_{S_{1}}\right)$ также не лог-канонична в точке $P$ по теореме 2.19 . Но $\left.\Omega\right|_{S_{1}} \sim_{\mathbb{Q}}-K_{S_{1}}$, что невозможно (см. пример 4.4).

Лемма 8.4. Пусть $I(X)=3.4$. Тогда $\operatorname{lct}(X)=1 / 2$. 
ДоказАтельство. Пусть $O$ - точка на $\mathbb{P}^{2}$. Тогда многообразие $X$ включается в коммутативную диаграмму

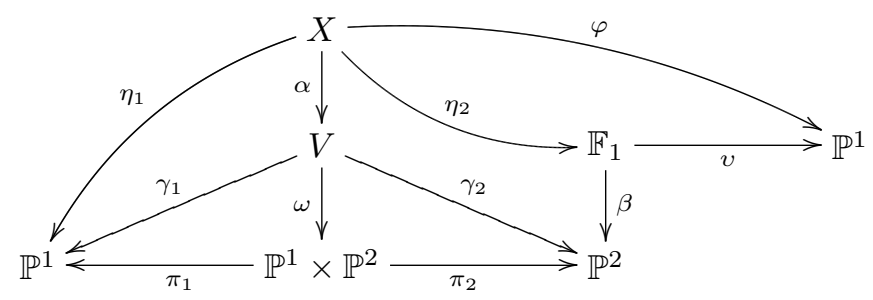

где $\pi_{i}$ и $v$ - естественные проекции, $\omega$ - двойное накрытие, разветвленное в дивизоре $B \subset \mathbb{P}^{1} \times \mathbb{P}^{2}$ бистепени $(2,2)$, морфизм $\gamma_{1}$ - расслоение на квадрики, $\gamma_{2}$ и $\eta_{2}$ - расслоения на коники, $\beta$ - раздутие точки $O$, морфизм $\alpha$ - раздутие неособой кривой, являющейся слоем расслоения $\gamma_{2}$ над точкой $O$, морфизм $\eta_{1}$ - расслоение на поверхности дель Пеццо степени 6 , а $\varphi$ - расслоение на поверхности дель Пеццо степени 4.

Пусть $H$ - общий слой расслоения $\eta_{1}$, а $S$ - общий слой расслоения $\varphi$. Тогда $-K_{X} \sim H+2 S+E$, где $E-$ исключительный дивизор раздутия $\alpha$. В частности, $\operatorname{lct}(X) \leqslant 1 / 2$.

Предположим, что $\operatorname{lct}(X)<1 / 2$. Тогда найдется эффективный $\mathbb{Q}$-дивизор $D \sim_{\mathbb{Q}}-K_{X}$ такой, что лог-пара $(X, \lambda D)$ не лог-канонична при некотором $\lambda<1 / 2$. Заметим, что $\operatorname{LCS}(X, \lambda D) \subseteq E$, так как $\alpha(D) \sim_{\mathbb{Q}}-K_{V}$ и $\operatorname{lct}(V)=1 / 2$ по лемме 7.7.

Пусть $\Gamma$ - слой расслоения $\eta_{2}$ такой, что $\Gamma \cap \operatorname{LCS}(X, \lambda D) \neq \varnothing$. Тогда $\Gamma \subseteq$ $\operatorname{LCS}(X, \lambda D) \subseteq E$ по теореме 2.27. Следовательно, лог-пара $\left(H,\left.\lambda D\right|_{H}\right)$ не логканонична в точках пересечения $H \cap \Gamma$. Но $\left.D\right|_{H} \sim_{\mathbb{Q}}-K_{H}$ и $\operatorname{lct}(H)=1 / 2$, так как $H$ - поверхность дель Пеццо степени 6 , противоречие.

Лемма 8.5. Пусть $\rightrightarrows(X)=3.5$ и $X$ общее. Тогда $\operatorname{lct}(X)=1 / 2$.

ДокАЗАтельство. Существует бирациональный морфизм $\alpha: X \rightarrow \mathbb{P}^{1} \times \mathbb{P}^{2}$, стягивающий поверхность $E \subset X$ на кривую $C \subset \mathbb{P}^{1} \times \mathbb{P}^{2}$ бистепени $(5,2)$. Пусть $\pi_{1}: \mathbb{P}^{1} \times \mathbb{P}^{2} \rightarrow \mathbb{P}^{1}$ и $\pi_{2}: \mathbb{P}^{1} \times \mathbb{P}^{2} \rightarrow \mathbb{P}^{2}$ - естественные проекции. Рассмотрим дивизор $Q \in\left|\pi_{2}^{*}\left(\mathscr{O}_{\mathbb{P}^{1}}(2)\right)\right|$ такой, что $C \subset Q$. Пусть $H_{1}-$ общий слой расслоения $\pi_{1}$, a $H_{2}$ - дивизор из линейной системы $\left|\pi_{2}^{*}\left(\mathscr{O}_{\mathbb{P}^{1}}(1)\right)\right|$. Тогда $-K_{X} \sim 2 \bar{H}_{1}+\bar{H}_{2}+\bar{Q}$, где $\bar{H}_{1}, \bar{H}_{2}, \bar{Q} \subset X$ - собственные прообразы дивизоров $H_{1}, H_{2}, Q$ соответственно. В частности, $\operatorname{lct}(X) \leqslant 1 / 2$.

Предположим, что многообразие $X$ удовлетворяет следующему условию общности: любой слой $F$ отображения $\pi_{1} \circ \alpha$ имеет не более одной обыкновенной двойной особенности.

Допустим, что $\operatorname{lct}(X)<1 / 2$. Тогда найдется эффективный $\mathbb{Q}$-дивизор $D \sim_{\mathbb{Q}}$ $-K_{X}$ такой, что лог-пара $(X, \lambda D)$ не является лог-канонической при некотором $\lambda<1 / 2$.

Пусть $S \subset X-$ неприводимая поверхность. Положим $D=\mu S+\Omega$, где $\Omega-$ эффективный $\mathbb{Q}$-дивизор такой, что $S$ не содержится в $\operatorname{Supp}(\Omega)$. Тогда лог-пара $\left(\bar{H}_{1},\left.(1 / 2)(\mu S+\Omega)\right|_{\bar{H}_{1}}\right)$ лог-канонична (см. пример 1.10). Таким образом, либо $\mu \leqslant 2$, либо $S$ является слоем отображения $\pi_{1} \circ \alpha$. 
Пусть $\Gamma \cong \mathbb{P}^{1}-$ общий слой расслоения на коники $\pi_{2} \circ \alpha$. Тогда

$$
2=D \cdot \Gamma=\mu S \cdot \Gamma+\Omega \cdot \Gamma \geqslant \mu S \cdot \Gamma
$$

откуда следует неравенство $\mu \leqslant 2$ в случае, когда $S$ является слоем отображения $\pi_{1} \circ \alpha$.

Мы видим, что множество $\mathbb{L} \mathbb{C} S(X, \lambda D)$ не содержит поверхностей. Применяя лемму 2.25 к отображению $\pi_{1} \circ \alpha$, получаем противоречие с примером 4.4 .

Лемма 8.6. Пусть $I(X)=3.6$ и $X$ общее. Тогда $\operatorname{lct}(X)=1 / 2$.

ДокАЗАТЕЛЬство. Пусть $\varepsilon: V \rightarrow \mathbb{P}^{3}$ - раздутие прямой $L \subset \mathbb{P}^{3}$. Тогда

$$
V \cong \mathbb{P}\left(\mathscr{O}_{\mathbb{P}^{1}} \oplus \mathscr{O}_{\mathbb{P}^{1}} \oplus \mathscr{O}_{\mathbb{P}^{1}}(1)\right)
$$

и $V$ имеет естественную структуру $\mathbb{P}^{2}$-расслоения $\eta: V \rightarrow \mathbb{P}^{1}$. Существует нормальная эллиптическая кривая $C \subset \mathbb{P}^{3}$ степени 4 такая, что $L \cap C=\varnothing$, и многообразие $X$ включается в коммутативную диаграмму

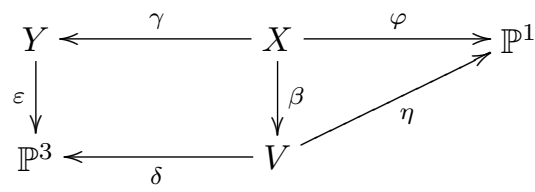

где $\delta$ - раздутие кривой $C, \beta$ - раздутие собственного прообраза прямой $L, \gamma-$ раздутие собственного прообраза кривой $C$, а $\varphi$ - расслоение на поверхности дель Пеццо степени 5.

Предположим, что $X$ удовлетворяет следующему условию общности: любой слой $F$ отображения $\varphi$ имеет не более одной обыкновенной двойной особенности.

Пусть $E$ и $G$ - исключительные дивизоры раздутий $\beta$ и $\gamma$ соответственно; пусть $H \subset \mathbb{P}^{3}$ - общая плоскость, проходящая через прямую $L$, а $Q \subset \mathbb{P}^{3}$ квадрика, проходящая через кривую $C$. Тогда $-K_{X} \sim 2 \bar{H}+\bar{Q}+E$, где $\bar{H} \subset X \supset \bar{Q}$ - собственные прообразы поверхностей $H$ и $Q$ соответственно. В частности, $\operatorname{lct}(X) \leqslant 1 / 2$.

Предположим, что $\operatorname{lct}(X)<1 / 2$. Тогда найдется эффективный $\mathbb{Q}$-дивизор $D \sim_{\mathbb{Q}}-K_{X}$ такой, что лог-пара $(X, \lambda D)$ не является лог-канонической при некотором $\lambda<1 / 2$.

Из леммы 7.11 следует, что $\operatorname{lct}(V)=1 / 2$. Отсюда $\operatorname{LCS}(X, \lambda D) \subseteq G$. Заметим, что любой слой расслоения $\varphi$ - поверхность дель Пеццо степени 5 , имеющая не более одной обыкновенной двойной особенности. Таким образом, применяя лемму 2.25 к отображению $\varphi$, получаем противоречие с примером 4.3.

Лемма 8.7. Пусть $\beth(X)=3.7$ и $X$ общее. Тогда $\operatorname{lct}(X)=1 / 2$.

ДоказАтельство. Пусть $W$ - дивизор бистепени $(1,1)$ на $\mathbb{P}^{2} \times \mathbb{P}^{2}$. Тогда $-K_{W} \sim 2 H$, где $H$ - дивизор Картье на $W$. Многообразие $X$ включается 
в коммутативную диаграмму

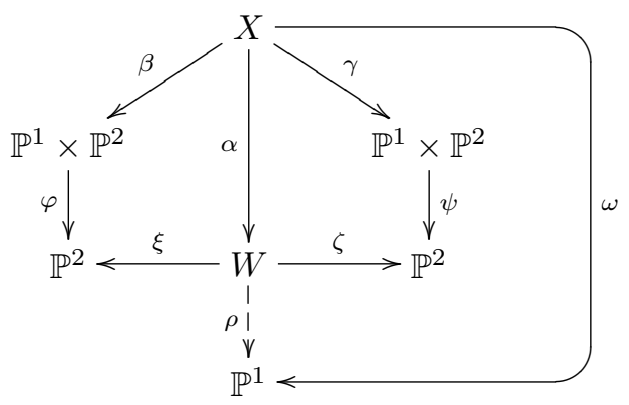

где $\varphi$ и $\psi$ - естественные проекции, $\alpha$ - раздутие неособой кривой $C \subset W$ такой, что

$$
C=H_{1} \cap H_{2}
$$

где $H_{1} \neq H_{2}$ - дивизоры из линейной системы $|H|$, отображение $\rho$ задается пучком, порожденным $H_{1}$ и $H_{2}, \omega$ - расслоение на поверхности дель Пеццо степени 6 , морфизмы $\zeta$ и $\xi$ являются $\mathbb{P}^{1}$-расслоениями, а бирациональные морфизмы $\beta$ и $\gamma$ стягивают поверхности $\bar{M}_{1} \subset X \supset \bar{M}_{2}$ такие, что $\varphi \circ \beta\left(\bar{M}_{1}\right)=\xi(C)$ и $\psi \circ \gamma\left(\bar{M}_{2}\right)=\zeta(C)$.

Заметим, что $\operatorname{lct}(X) \leqslant 1 / 2$, так как $-K_{X} \sim 2 \bar{H}_{1}+E$, где $\bar{H}_{1} \subset X-$ собственный прообраз поверхности $H_{1}$, а $E$ - исключительный дивизор раздутия $\alpha$.

Будем считать $X$ настолько общим, что все особые слои расслоения $\omega$ удовлетворяют условиям леммы 4.5.

Допустим, что $\operatorname{lct}(X)<1 / 2$. Тогда найдется эффективный $\mathbb{Q}$-дивизор $D \sim_{\mathbb{Q}}$ $-K_{X}$ такой, что лог-пара $(X, \lambda D)$ не лог-канонична при некотором $\lambda<1 / 2$. Тогда $\operatorname{LCS}(X, \lambda D) \subseteq E$, так как $\operatorname{lct}(W)=1 / 2$ по теореме 6.1. Применяя лемму 2.25 , получаем, что $\operatorname{LCS}(X, \lambda D) \subseteq E \cap F$, где $F$ - особый слой расслоения $\omega$. Напомним, что $F$ - поверхность дель Пеццо степени 6 . Положим $D=\mu F+\Omega$, где $\Omega$ - эффективный $\mathbb{Q}$-дивизор такой, что $F \not \subset \operatorname{Supp}(\Omega)$. Тогда выполнено соотношение $\left.\Omega\right|_{F} \sim_{\mathbb{Q}}-K_{F}$ и поверхность $F$ неособа вдоль кривой $E \cap F$. При этом по теореме 2.19 лог-пара $\left(F,\left.\lambda \Omega\right|_{F}\right)$ не лог-канонична в некоторой точке $P \in E \cap F$, что невозможно по лемме 4.5 .

ЗАмЕчАНИЕ 8.8. В обозначениях и предположениях леммы 8.7 имеем

$$
\operatorname{LCS}(X, \lambda D) \subseteq E \cap F
$$

где $F$ - особый слой расслоения $\omega$. Применяя теорему 2.27 к отображениям $\varphi$ и $\psi$ и используя лемму 2.28 , получаем, что $\operatorname{LCS}(X, \lambda D) \subseteq E \cap F \cap \bar{M}_{1} \cap \bar{M}_{2}$. Независимо от того, насколько особа поверхность $F$, если многообразие $X$ достаточно общее, то $E \cap F \cap \bar{M}_{1} \cap \bar{M}_{2}=\varnothing$, т. е. утверждение леммы 8.7 может быть доказано и при другом условии общности.

Лемма 8.9. Пусть $I(X)=3.8$ и $X$ общее. Тогда $\operatorname{lct}(X)=1 / 2$.

ДокАЗАТЕЛЬство. Пусть $\pi_{1}: \mathscr{F}_{1} \times \mathbb{P}^{2} \rightarrow \mathscr{F}_{1}$ и $\pi_{2}: \mathscr{F}_{1} \times \mathbb{P}^{2} \rightarrow \mathbb{P}^{2}$ - естественные проекции. Тогда $X \in\left|\left(\alpha \circ \pi_{1}\right)^{*}\left(\mathscr{O}_{\mathbb{P}^{2}}(1)\right) \otimes \pi_{2}^{*}\left(\mathscr{O}_{\mathbb{P}^{2}}(2)\right)\right|$, где $\alpha: \mathscr{F}_{1} \rightarrow \mathbb{P}^{2}-$ 
раздутие точки. Пусть $H$ - дивизор из линейной системы $\left|\pi_{2}^{*}\left(\mathscr{O}_{\mathbb{P}^{2}}(1)\right)\right|$. Тогда $-K_{X} \sim E+2 L+H$, где $E \subset X \supset L$ - неприводимые поверхности такие, что $\pi_{1}(E) \subset \mathbb{F}_{1}$ является исключительной кривой раздутия $\alpha$, а $\pi_{1}(L) \subset \mathbb{F}_{1}$ является слоем естественной проекции $\mathbb{F}_{1} \rightarrow \mathbb{P}^{2}$. В частности, $\operatorname{lct}(X) \leqslant 1 / 2$.

Проекция $\pi_{1}$ индуцирует расслоение $\varphi: X \rightarrow \mathbb{P}^{1}$ на поверхности дель Пеццо степени 5.

Предположим, что многообразие $X$ удовлетворяет следующему условию общности: любой слой $F$ отображения $\varphi$ имеет не более одной обыкновенной двойной особенности.

Допустим, что $\operatorname{lct}(X)<1 / 2$. Тогда найдется эффективный $\mathbb{Q}$-дивизор $D \sim_{\mathbb{Q}}$ $-K_{X}$ такой, что лог-пара $(X, \lambda D)$ не лог-канонична при некотором $\lambda<1 / 2$.

Применяя лемму 2.25 к расслоению $\varphi$, получаем противоречие с примеpom 4.3 .

ЛЕмма 8.10. Пусть $\beth(X)=3.9$. Тогда $\operatorname{lct}(X)=1 / 3$.

ДоКАЗАТЕЛьСтво. Пусть $O_{i}$ - особая точка многообразия $V_{i} \cong \mathbb{P}(1,1,1,2)$, где $i=1,2$. Выберем гладкую поверхность $O_{1} \notin S_{1} \in\left|\mathscr{O}_{\mathbb{P}(1,1,1,2)}(2)\right|$, и пусть $C_{1} \subset S_{1} \cong \mathbb{P}^{2}$ - неособая квартика. Тогда $X$ включается в коммутативную диаграмму

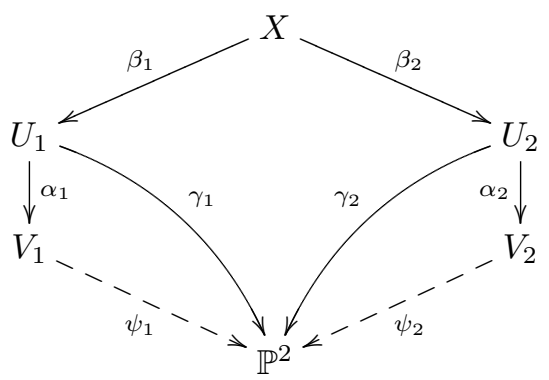

где $\psi_{i}$ - естественная проекция, $\alpha_{i}$ - (взвешенное) раздутие точки $O_{i}$ с весами $(1,1,1)$, морфизм $\gamma_{i}$ является $\mathbb{P}^{1}$-расслоением, а $\beta_{i}$ - бирациональный морфизм, стягивающий поверхность $\mathbb{P}^{1} \times C_{1} \cong G_{i} \subset X$ на гладкую кривую $C_{1} \cong C_{i} \subset U_{i}$.

Пусть $E_{i} \subset X$ - собственный прообраз исключительного дивизора раздутия $\alpha_{i}$. Тогда дивизоры

$$
S_{1}=\alpha_{1} \circ \beta_{1}\left(E_{2}\right) \subset V_{1} \cong \mathbb{P}(1,1,1,2) \cong V_{2} \supset \alpha_{2} \circ \beta_{2}\left(E_{1}\right)
$$

лежат в линейной системе $\left|\mathscr{O}_{\mathbb{P}(1,1,1,2)}(2)\right|$ и содержат кривые $C_{1}$ и $C_{2}$ соответственно. При этом дивизоры

$$
\alpha_{1} \circ \beta_{1}\left(G_{2}\right) \subset V_{1} \cong \mathbb{P}(1,1,1,2) \cong V_{2} \supset \alpha_{2} \circ \beta_{2}\left(G_{1}\right)
$$

лежат в линейной системе $\left|\mathscr{O}_{\mathbb{P}(1,1,1,2)}(4)\right|$ и содержат $O_{1} \cup C_{1}$ и $O_{2} \cup C_{2}$ соответственно.

Пусть $\bar{H} \subset X$ - собственный прообраз общей поверхности из линейной системы $\left|\mathscr{O}_{\mathbb{P}(1,1,1,2)}(1)\right|$. Тогда $-K_{X} \sim 3 \bar{H}+E_{2}+E_{1}$, откуда следует неравенство $\operatorname{lct}(X) \leqslant 1 / 3$.

Предположим, что $\operatorname{lct}(X)<1 / 3$. Тогда найдется эффективный $\mathbb{Q}$-дивизор

$$
D \sim_{\mathbb{Q}}-K_{X} \sim_{\mathbb{Q}} \frac{5}{2}\left(G_{1}+G_{2}\right)-5\left(E_{1}+E_{2}\right)
$$


такой, что лог-пара $(X, \lambda D)$ не лог-канонична при некотором $\lambda<1 / 3$. Положим $D=\mu_{1} E_{1}+\mu_{2} E_{2}+\Omega$, где $\Omega$ - эффективный $\mathbb{Q}$-дивизор на $X$ такой, что $E_{1} \not \subset \operatorname{Supp}(\Omega) \nsupseteq E_{2}$.

Пусть $\Gamma$ - общий слой расслоения на коники $\gamma_{1} \circ \beta_{1}$. Тогда

$$
2=\Gamma \cdot D=\Gamma \cdot\left(\mu_{1} E_{1}+\mu_{2} E_{2}+\Omega\right)=\mu_{1}+\mu_{2}+\Gamma \cdot \Omega \geqslant \mu_{1}+\mu_{2},
$$

и без ограничения общности можно считать, что $\mu_{1} \leqslant \mu_{2}$. Тогда $\mu_{1} \leqslant 1$.

Допустим, что существует поверхность $S \in \mathbb{L} \mathbb{C}(X, \lambda D)$. Тогда $S \neq E_{1}$ и $S \neq G_{1}$, так как $\alpha_{2} \circ \beta_{2}\left(G_{1}\right) \in\left|\mathscr{O}_{\mathbb{P}(1,1,1,2)}(4)\right|$ и $\alpha_{2} \circ \beta_{2}(D) \in\left|\mathscr{O}_{\mathbb{P}(1,1,1,2)}(5)\right|$. Следовательно, $S \cap E_{1} \neq \varnothing$. Но

$$
-\left.\frac{1}{3} K_{E_{1}} \sim_{\mathbb{Q}} D\right|_{E_{1}}=-\frac{2 \mu_{1}}{3} K_{E_{1}}+\left.\Omega\right|_{E_{1}}
$$

и $E_{1} \cong \mathbb{P}^{2}$, что невозможно по теореме 2.19 , так как $\lambda<1 / 3=\operatorname{lct}\left(\mathbb{P}^{2}\right)$.

Таким образом, множество $\mathbb{L} \mathbb{C} S(X, \lambda D)$ не содержит поверхностей. Выберем какую-нибудь точку $P \in \operatorname{LCS}(X, \lambda D)$. Предположим, что $P \notin G_{1}$. Пусть $Z$ - слой отображения $\gamma_{1}$, проходящий через точку $\beta_{1}(P)$. Тогда $Z \subseteq$ $\operatorname{LCS}\left(U_{1}, \lambda \beta_{1}(D)\right)$ по теореме 2.27. Положим $\bar{E}_{1}=\beta_{1}\left(E_{1}\right)$. Тогда $Z \cap \bar{E}_{1} \in$ $\operatorname{LCS}\left(\bar{E}_{1},\left.\lambda \Omega\right|_{\bar{E}_{1}}\right)$ по теореме 2.19 , что невозможно по лемме 2.8 , так как $\mu_{1} \leqslant 1$. Таким образом, $\operatorname{LCS}(X, \lambda D) \subsetneq G_{1}$.

Допустим, что $\operatorname{LCS}(X, \lambda D) \subseteq G_{1} \cap G_{2}$. Тогда $|\operatorname{LCS}(X, \lambda D)|=1$ по лемме 2.14 и теореме 2.7. При этом

$\operatorname{LCS}(X, \lambda D) \cup \bar{H} \subseteq \operatorname{LCS}\left(X, \lambda D+\frac{1}{3}\left(E_{2}+E_{2}\right)+\bar{H}\right) \subset \operatorname{LCS}(X, \lambda D) \cup \bar{H} \cup E_{1} \cup E_{1}$,

что противоречит теореме 2.7, так как $\bar{H}$ выбрана в линейной системе $\left|\left(\beta_{1} \circ \gamma_{1}\right)^{*}\left(\mathscr{O}_{\mathbb{P}^{2}}(1)\right)\right|$ общим образом и

$$
\lambda D+\frac{1}{3}\left(E_{2}+E_{2}\right)+\bar{H} \sim_{\mathbb{Q}}\left(\lambda-\frac{1}{3}\right) K_{X} .
$$

Таким образом, $G_{1} \supsetneq \mathbb{L} \mathbb{C} S(X, \lambda D) \not \subset G_{1} \cap G_{2}$. Поэтому

$$
\varnothing \neq \operatorname{LCS}\left(U_{2}, \lambda \beta_{2}(D)\right) \subsetneq \beta_{2}\left(G_{1}\right),
$$

и из теорем 2.7 и 2.27 следует, что $\operatorname{LCS}\left(U_{2}, \lambda \beta_{2}(D)\right)=L$ для некоторого слоя $L$ расслоения $\gamma_{2}$.

Пусть $B$ - общий дивизор в линейной системе $\left|\alpha_{2}^{*}\left(\mathscr{O}_{\mathbb{P}(1,1,1,2)}(2)\right)\right|$. Тогда $\left.\beta_{2}(D)\right|_{B} \sim_{\mathbb{Q}} \mathscr{O}_{\mathbb{P}^{2}}(5)$ и $B \cong \mathbb{P}^{2}$. При этом $\operatorname{LCS}\left(B,\left.\lambda \beta_{2}(D)\right|_{B}\right)=L \cap B$ и $|L \cap B|=1$, что противоречит лемме 2.8 .

Лемма 8.11. Пусть $\beth(X)=3.10$. Тогда $\operatorname{lct}(X)=1 / 2$.

ДоказАтельство. Пусть $Q \subset \mathbb{P}^{4}$ - неособая квадрика, а $C_{1} \subset Q \supset C_{2}-$ непересекающиеся (неприводимые) коники. Тогда многообразие $X$ включается 
в коммутативную диаграмму

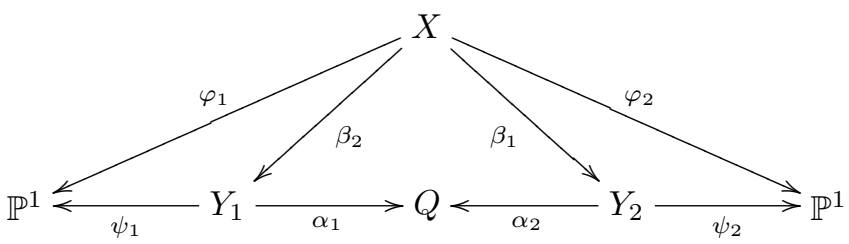

где $\alpha_{i}$ - раздутие коники $C_{i}$, морфизм $\beta_{i}$ - раздутие собственного прообраза коники $C_{i}$, морфизм $\psi_{i}$ является расслоением на квадрики, а морфизм $\varphi_{i}-$ расслоением на поверхности дель Пеццо степени 6.

Пусть $E_{i}$ - исключительный дивизор раздутия $\beta_{i}$, а $H_{i}$ - достаточно общее гиперплоское сечение квадрики $Q$, проходящее через конику $C_{i}$. Тогда $-K_{X} \sim$ $\bar{H}_{1}+2 \bar{H}_{2}+E_{2}$, где $\bar{H}_{i} \subset X-$ собственный прообраз дивизора $H_{i}$. В частности, $\operatorname{lct}(X) \leqslant 1 / 2$.

Предположим, что $\operatorname{lct}(X)<1 / 2$. Тогда найдется эффективный $\mathbb{Q}$-дивизор $D \sim_{\mathbb{Q}}-K_{X}$ такой, что лог-пара $(X, \lambda D)$ не является лог-канонической при некотором $\lambda<1 / 2$.

Используя пример 1.10 и применяя лемму 2.25, получаем, что $\operatorname{LCS}(X, \lambda D) \subseteq$ $S_{1} \cap S_{2}$, где $S_{i}$ - особый слой расслоения $\varphi_{i}$. Следовательно, $\mathbb{L} \mathbb{C} \mathbb{S}(X, \lambda D)$ не содержит поверхностей.

Из теоремы 2.7 следует, что либо $\operatorname{LCS}(X, \lambda D)$ является точкой на $E_{1} \cup E_{2}$, либо

$$
\operatorname{LCS}(X, \lambda D) \cap\left(X \backslash\left(E_{1} \cup E_{2}\right)\right) \neq \varnothing,
$$

т. е. по лемме 2.10 можно считать, что $\operatorname{LCS}(X, \lambda D)$ является точкой на $E_{1}$.

Так как ограничение морфизма $\beta_{2}$ на $X \backslash E_{2}$ является изоморфизмом, то

$$
P \in \operatorname{LCS}\left(Y_{1}, \lambda \beta_{2}(D)\right) \subset P \cup \beta_{2}\left(E_{2}\right)
$$

для некоторой точки $P \in E_{1}$. Следовательно, $\operatorname{LCS}\left(Y_{1}, \lambda \beta_{2}(D)\right)=P$ по теореме 2.7, так как $P \notin \beta_{2}\left(E_{2}\right)$.

Пусть $H$ - общее гиперплоское сечение квадрики $Q$. Тогда выполнены эквивалентности $-K_{Y_{1}} \sim \widetilde{H}_{1}+2 \widetilde{H} \sim_{\mathbb{Q}} \beta_{2}(D)$, где $\widetilde{H} \subset Y_{1} \supset \widetilde{H}_{1}-$ собственные прообразы поверхностей $H$ и $H_{1}$ соответственно. При этом

$$
\operatorname{LCS}\left(Y_{1}, \lambda \beta_{2}(D)+\frac{1}{2}\left(\widetilde{H}_{1}+2 \widetilde{H}\right)\right)=P \cup \widetilde{H},
$$

что невозможно по теореме 2.7 , так как $\lambda<1 / 2$.

Лемма 8.12. Пусть $I(X)=3.11$. Тогда $\operatorname{lct}(X)=1 / 2$.

ДоказАтельство. Пусть $O \in \mathbb{P}^{3}$ - некоторая точка, морфизм $\delta: V_{7} \rightarrow \mathbb{P}^{3}$ является раздутием точки $O$ и $E$ - исключительный дивизор раздутия $\delta$. Тогда $V_{7} \cong \mathbb{P}\left(\mathscr{O}_{\mathbb{P}^{2}} \oplus \mathscr{O}_{\mathbb{P}^{2}}(1)\right)$, на $V_{7}$ имеется естественная структура $\mathbb{P}^{1}$-расслоения $\eta: V_{7} \rightarrow \mathbb{P}^{2}$ и $E$ является сечением $\eta$. Существует нормальная эллиптическая 
кривая $O \in C \subset \mathbb{P}^{3}$ степени 4 такая, что многообразие $X$ включается в коммутативную диаграмму

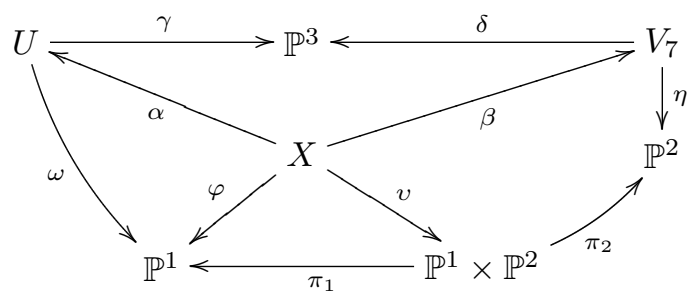

где $\pi_{1}$ и $\pi_{2}$ - естественные проекции, морфизм $\gamma$ стягивает поверхность

$$
C \times \mathbb{P}^{1} \cong G \subset U
$$

на кривую $C$, морфизм $\alpha$ - раздутие слоя морфизма $\gamma$ над точкой $O \in \mathbb{P}^{3}$, морфизм $\beta$ - раздутие собственного прообраза кривой $C$, морфизм $\omega$ - расслоение на квадрики, $\varphi$ - расслоение на поверхности дель Пеццо степени 7 , а $v$ стягивает поверхность

$$
C \times \mathbb{P}^{1} \cong F \subset X
$$

на эллиптическую кривую $Z \subset \mathbb{P}^{1} \times \mathbb{P}^{2}$ такую, что $-K_{\mathbb{P}^{1} \times \mathbb{P}^{2}} \cdot Z=13$ и $Z \cong C$.

Пусть $H_{1}$ - общий слой расслоения $\varphi$, а $H_{2}$ - общий дивизор в линейной системе $\left|(\eta \circ \beta)^{*}\left(\mathscr{O}_{\mathbb{P}^{2}}(1)\right)\right|$. Тогда $-K_{X} \sim H_{1}+2 H_{2}$, откуда следует неравенство $\operatorname{lct}(X) \leqslant 1 / 2$.

Предположим, что $\operatorname{lct}(X)<1 / 2$. Тогда найдется эффективный $\mathbb{Q}$-дивизор $D \sim_{\mathbb{Q}}-K_{X}$ такой, что особенности лог-пары $(X, \lambda D)$ не являются лог-каноническими при некотором $\lambda<1 / 2$. Заметим, что $\operatorname{LCS}(X, \lambda D) \subseteq \bar{E}$, где $\bar{E}-$ исключительный дивизор раздутия $\alpha$, так как $\operatorname{lct}(U)=1 / 2$ по лемме 7.11.

Пусть $\Gamma \cong \mathbb{P}^{2}-$ общий слой отображения $\pi_{2} \circ v$. Тогда

$$
2=-K_{X} \cdot \Gamma=D \cdot \Gamma=2 \bar{E} \cdot \Gamma
$$

откуда следует, что $\bar{E} \not \subset \operatorname{LCS}(X, \lambda D)$. По лемме 2.25 , примененной к лог-паpe $\left(V_{7}, \lambda \beta(D)\right)$, получаем, что $\operatorname{LCS}(X, \lambda D) \subseteq \bar{E} \cap G$. Применяя лемму 2.28 к лог-паре $\left(\mathbb{P}^{1} \times \mathbb{P}^{2}, \lambda v(D)\right)$, получаем, что $\operatorname{LCS}(X, \lambda D)=\bar{E} \cap F \cap G$, причем $|\bar{E} \cap F \cap G|=1$. Следовательно,

$$
\operatorname{LCS}\left(X, \lambda D+H_{2}\right)=\operatorname{LCS}(X, \lambda D) \cup H_{2}
$$

и $H_{2} \cap \operatorname{LCS}(X, \lambda D)=\varnothing$. Но дивизор

$$
-\left(K_{X}+\lambda D+H_{2}\right)=\left(\lambda-\frac{1}{2}\right) K_{X}+\frac{1}{2} H_{1}
$$

обилен, что невозможно по теореме 2.7 .

Лемма 8.13. Пусть $I(X)=3.12$. Тогда $\operatorname{lct}(X)=1 / 2$.

ДокАзАтельство. Пусть $\varepsilon: V \rightarrow \mathbb{P}^{3}$ - раздутие прямой $L \subset \mathbb{P}^{3}$. На $V$ имеется естественная структура $\mathbb{P}^{2}$-расслоения $\eta: V \rightarrow \mathbb{P}^{1}$. Можно выбрать 
скрученную кубику $C \subset \mathbb{P}^{3}$, не пересекающую прямую $L$, такую, что многообразие $X$ включается в коммутативную диаграмму

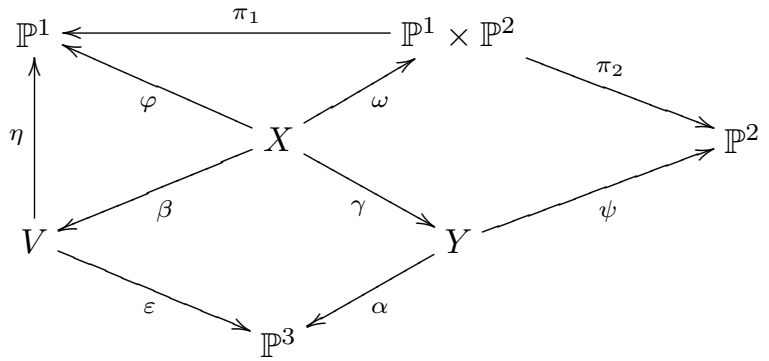

где $\alpha$ и $\beta$ - раздутия кривой $C$ и ее собственного прообраза соответственно, $\gamma$ - раздутие собственного прообраза прямой $L$, морфизм $\psi$ является $\mathbb{P}^{1}$-расслоением, $\omega$ - стягивание на кривую поверхности $F \subset X$ такой, что $C \cup L \subset$ $\alpha \circ \gamma(F)$, и $\alpha \circ \gamma(F)$ состоит из секущих прямых к кривой $C \subset \mathbb{P}^{3}$, пересекающих прямую $L$; морфизм $\varphi$ - расслоение на поверхности дель Пеццо степени 6 , а морфизмы $\pi_{1}$ и $\pi_{2}$ - естественные проекции.

Пусть $E$ и $G$ - исключительные дивизоры раздутий $\beta$ и $\gamma$ соответственно, $Q \subset \mathbb{P}^{3}$ - общая квадрика, проходящая через кривую $C, H \subset \mathbb{P}^{3}$ - общая плоскость, проходящая через прямую $L$. Тогда выполнено соотношение $-K_{X} \sim$ $\bar{Q}+2 \bar{H}+G$, где $\bar{Q} \subset X \supset \bar{H}-$ собственные прообразы дивизоров $Q \subset \mathbb{P}^{3} \supset H$ соответственно. В частности, $\operatorname{lct}(X) \leqslant 1 / 2$.

Предположим, что $\operatorname{lct}(X)<1 / 2$. Тогда найдется эффективный $\mathbb{Q}$-дивизор $D \sim_{\mathbb{Q}}-K_{X}$ такой, что лог-пара $(X, \lambda D)$ не лог-канонична при некотором $\lambda<1 / 2$. Заметим, что $\operatorname{LCS}(X, \lambda D) \subset G$, так как $\operatorname{lct}(Y)=1 / 2$ по лемме 7.13. Применяя теорему 2.27 к расслоению $\varphi$, получаем, что $\operatorname{LCS}(X, \lambda D) \subset$ $G \cap S_{\varphi}$, где $S_{\varphi}-$ особый слой расслоения $\varphi$ (см. пример 1.10). Следовательно, $\operatorname{LCS}(X, \lambda D) \subset G \cap S_{\varphi} \cap F$ по теореме 2.27 , примененной к лог-паре $\left(\mathbb{P}^{1} \times \mathbb{P}^{2}\right.$, $\lambda \omega(D))$ и $\mathbb{P}^{1}$-расслоению $\pi_{2}$.

Пусть $Z_{1} \cong \mathbb{P}^{1}$ - сечение естественной проекции $\mathbb{P}^{1} \times \mathbb{P}^{1} \cong G \rightarrow L \cong \mathbb{P}^{1}$ с самопересечением $Z_{1} \cdot Z_{1}=0$, а $Z_{2}-$ слой этой проекции. Тогда $\left.F\right|_{G} \sim$ $Z_{1}+3 Z_{2}$ и $\left.S_{\varphi}\right|_{G} \sim Z_{1}$. При этом кривая $F \cap G$ неприводима. Таким образом, $\left|G \cap F \cap S_{\varphi}\right|<+\infty$, откуда по теореме 2.7 следует, что множество $\operatorname{LCS}(X, \lambda D)$ состоит из одной точки $P \in G$.

Лог-пара $(V, \lambda \beta(D))$ не лог-канонична. Так как ограничение морфизма $\beta$ на множество $X \backslash E$ является изоморфизмом, то

$$
\beta(P) \in \operatorname{LCS}(V, \lambda \beta(D)) \subseteq \beta(P) \cup \beta(E),
$$

откуда по теореме 2.7 следует, что $\operatorname{LCS}(V, \lambda \beta(D))=\beta(P)$. Пусть $H \subset \mathbb{P}^{3}-$ общая плоскость. Тогда

$$
\operatorname{LCS}\left(V, \lambda \beta(D)+\frac{1}{2}\left(\widetilde{H}_{1}+3 \widetilde{H}\right)\right)=\beta(P) \cup \widetilde{H},
$$

где $\widetilde{H} \subset V \supset \widetilde{H}_{1}$ - собственные прообразы дивизоров $H \subset \mathbb{P}^{3} \supset H_{1}$ соответственно. При этом $-K_{V} \sim \widetilde{H}_{1}+3 \widetilde{H} \sim_{\mathbb{Q}} \beta(D)$, что противоречит теореме 2.7 , так как $\lambda<1 / 2$. 
Лемма 8.14. Пусть $I(X)=3.14$. Тогда $\operatorname{lct}(X)=1 / 2$.

ДоказАтельство. Пусть $P \in \mathbb{P}^{3}$ - некоторая точка и $\alpha: V_{7} \rightarrow \mathbb{P}^{3}$ - раздутие точки $P$. Тогда на $V_{7}$ имеется естественная структура $\mathbb{P}^{1}$-расслоения $\pi: V_{7} \rightarrow \mathbb{P}^{2}$.

Пусть $\zeta: Z \rightarrow \mathbb{P}(1,1,1,2)$ - раздутие особой точки на $\mathbb{P}(1,1,1,2)$. Тогда имеется естественное $\mathbb{P}^{1}$-расслоение $\varphi: Z \rightarrow \mathbb{P}^{2}$, поскольку $Z \cong \mathbb{P}\left(\mathscr{O}_{\mathbb{P}^{2}} \oplus \mathscr{O}_{\mathbb{P}^{2}}(2)\right)$.

Можно выбрать плоскость $\Pi \subset \mathbb{P}^{3}$ и неособую кубическую кривую $C \subset \Pi$ так, что $P \notin \Pi$, и многообразие $X$ включается в коммутативную диаграмму (см. [28; пример 3.6])

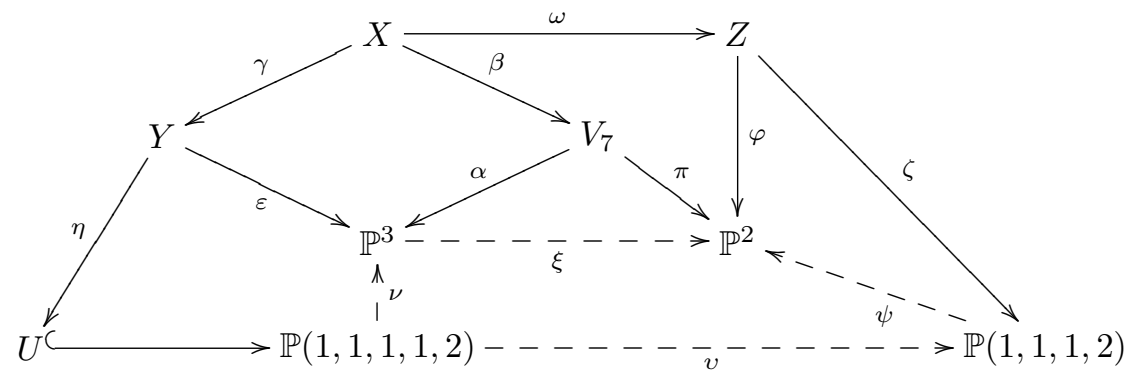

В диаграмме использованы следующие обозначения: морфизм $\varepsilon-$ раздутие кривой $C$; многообразие $U$ - гиперповерхность степени 3 в $\mathbb{P}(1,1,1,1,2)$; рациональное отображение $\xi$ - проекция из точки $P$; морфизм $\gamma$ - раздутие точки, лежащей над $P$; морфизм $\beta$ - раздутие собственного прообраза кривой $C$; морфизм $\eta$ стягивает собственный прообраз плоскости П в точку $\operatorname{Sing}(U)$, морфизм $\omega$ стягивает на кривую такую поверхность $R \subset X$, что поверхность $\beta \circ \alpha(R)$ является конусом над кривой $C$ с вершиной в точке $P$; рациональные отображения $\psi$ и $\nu$ - естественные проекции; рациональное отображение $v$ - проекция из точки.

Пусть $E$ и $G$ - исключительные дивизоры раздутий $\gamma$ и $\beta$ соответственно, а $\bar{H} \subset X$ - собственный прообраз общей плоскости в $\mathbb{P}^{3}$, проходящей через точку $P$. Тогда выполнено соотношение $-K_{X} \sim \bar{\Pi}+3 \bar{H}+G$, где $\bar{\Pi} \subset X-$ собственный прообраз плоскости П. Таким образом, $\operatorname{lct}(X) \leqslant 1 / 3$.

Предположим, что $\operatorname{lct}(X)<1 / 3$. Тогда найдется эффективный $\mathbb{Q}$-дивизор $D \sim_{\mathbb{Q}}-K_{X}$ такой, что лог-пара $(X, \lambda D)$ не является лог-канонической при некотором $\lambda<1 / 3$.

Пусть $\bar{L} \subset X-$ собственный прообраз общей прямой в $\mathbb{P}^{3}$, пересекающей кривую $C$. Тогда

$$
D \cdot \bar{L}=\bar{\Pi} \cdot \bar{L}+3 \bar{H} \cdot \bar{L}+G \cdot \bar{L}=3 \bar{H} \cdot \bar{L}=3,
$$

откуда следует, что множество $\mathbb{L} \mathbb{C} S(X, \lambda D)$ не содержит поверхностей, за исключением, быть может, $\bar{\Pi}$ и $E$.

Пусть Г - общий слой отображения $\pi \circ \beta$. Тогда

$$
D \cdot \Gamma=\bar{\Pi} \cdot \Gamma+3 \bar{H} \cdot \Gamma+G \cdot \Gamma=\bar{\Pi} \cdot \Gamma+G \cdot \Gamma=2,
$$

откуда следует, что множество $\mathbb{L} \mathbb{C} S(X, \lambda D)$ не содержит ни $\bar{\Pi}$, ни $E$. Таким образом, по лемме $2.9 \operatorname{LCS}(X, \lambda D) \subsetneq E \cup G$. 
Допустим, что $\operatorname{LCS}(X, \lambda D) \subseteq E$. Тогда $\varnothing \neq \operatorname{LCS}\left(V_{7}, \lambda \beta(D)\right) \subseteq \beta(E)$, что противоречит теореме 2.27 , так как $\beta(E)$ является сечением расслоения $\pi$. Таким образом, $\operatorname{LCS}(X, \lambda D) \subsetneq G$.

Применяя теорему 2.27 к лог-паре $(Z, \lambda \omega(D))$ и расслоению $\varphi$ и теорему 2.7 к лог-паре $(X, \lambda D)$, получаем, что $\operatorname{LCS}(X, \lambda D) \subseteq F$, где $F$ - некоторый слой естественной проекции $G \rightarrow \beta(G)$. Следовательно, $\varnothing \neq \operatorname{LCS}(Y, \lambda \gamma(D)) \subseteq \gamma(F)$, где $\gamma(F)$ - слой раздутия $\varepsilon$ над точкой кривой $C$.

Пусть $S \subset \mathbb{P}^{3}$ - общий конус над кривой $C$ и $O \in C$ - точка перегиба такая, что $\varepsilon \circ \gamma(F) \neq O$. Пусть $L \subset S$ - образующая конуса $S$, проходящая через точку $O$, а $H \subset \mathbb{P}^{3}$ - плоскость, касающаяся конуса $S$ вдоль прямой $L$. Так как $O$ - точка перегиба кривой $C$, то $\operatorname{mult}_{L}(S \cdot H)=3$. Пусть $\breve{S}, \breve{H}$ и $\breve{L}-$ собственные прообразы $S, H$ и $L$ на многообразии $Y$. Тогда

$$
\operatorname{LCS}\left(Y, \lambda \gamma(D)+\frac{2}{3}(\breve{S}+\breve{H})\right)=\operatorname{LCS}(Y, \lambda \gamma(D)) \cup \breve{L}
$$

из-за общности в выборе конуса $S$. Но $-K_{Y} \sim \breve{S}+\breve{H}$, что невозможно по теореме 2.7 .

Лемма 8.15. Пусть $I(X)=3.15$. Тогда $\operatorname{lct}(X)=1 / 2$.

ДоказАтельство. Пусть $Q \subset \mathbb{P}^{4}-$ неособая квадрика, $C \subset Q-$ (неособая) коника и $\varepsilon: V \rightarrow Q$ - раздутие коники $C \subset Q$. Имеется естественное отображение $\eta: V \rightarrow \mathbb{P}^{1}$, индуцированное проекцией $Q \rightarrow \mathbb{P}^{1}$ из двумерного линейного подпространства в $\mathbb{P}^{4}$, содержащего конику $C$. При этом общий слой отображения $\eta$ является неособой квадрикой в $\mathbb{P}^{3}$.

Выберем прямую $L \subset Q$ такую, что $L \cap C=\varnothing$. Имеет место коммутативная диаграмма

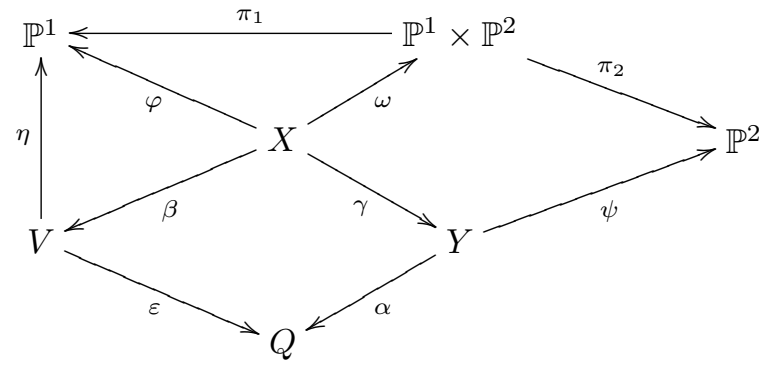

где $\alpha$ и $\beta$ - раздутия прямой $L \subset Q$ и ее собственного прообраза соответственно, $\gamma$ - раздутие собственного прообраза коники $C$, морфизм $\psi$ является $\mathbb{P}^{1}$-расслоением, $\omega$ стягивает на кривую такую поверхность $F \subset X$, что $C \cup L \subset \alpha \circ \gamma(F) \subset Q$, поверхность $\alpha \circ \gamma(F)$ заметается прямыми на $Q \subset \mathbb{P}^{4}$, пересекающими $L$ и $C$, морфизм $\varphi$ - расслоение на поверхности дель Пеццо степени 7 , а морфизмы $\pi_{1}$ и $\pi_{2}$ - естественные проекции.

Пусть $E_{1}$ и $E_{2}$ - исключительные дивизоры раздутий $\beta$ и $\gamma$ соответственно; пусть $H_{1}, H_{2} \subset Q$ - общие гиперплоские сечения, проходящие через кривые $L$ и $C$ соответственно. Тогда $-K_{X} \sim \bar{H}_{1}+2 \bar{H}_{2}+E_{2} \sim \bar{H}_{2}+2 \bar{H}_{1}+E_{1}$, где $\bar{H}_{1} \subset$ $X \supset \bar{H}_{2}-$ собственные прообразы дивизоров $H_{1} \subset Q \supset H_{2}$ соответственно. В частности, $\operatorname{lct}(X) \leqslant 1 / 2$. 
Предположим, что $\operatorname{lct}(X)<1 / 2$. Тогда найдется эффективный $\mathbb{Q}$-дивизор $D \sim_{\mathbb{Q}}-K_{X}$ такой, что лог-пара $(X, \lambda D)$ не является лог-канонической при некотором $\lambda<1 / 2$.

Пусть $S \subset X-$ неприводимая поверхность. Положим $D=\mu S+\Omega$, где $\Omega-$ эффективный $\mathbb{Q}$-дивизор такой, что $S \not \subset \operatorname{Supp}(\Omega)$. Тогда по лемме 4.9

$$
\operatorname{LCS}\left(\bar{H}_{2},\left.\frac{1}{2}(\mu S+\Omega)\right|_{\bar{H}_{2}}\right) \subset E_{1} \cap \bar{H}_{2} .
$$

Таким образом, если $\mu \leqslant 2$, то либо $S=E_{1}$, либо $S$ является слоем расслоения $\varphi$.

Пусть $\Gamma \cong \mathbb{P}^{1}-$ общий слой расслоения на коники $\psi \circ \gamma$. Тогда

$$
2=D \cdot \Gamma=\mu S \cdot \Gamma+\Omega \cdot \Gamma \geqslant \mu S \cdot \Gamma
$$

откуда следует, что $\mu \leqslant 2$, если $S=E_{1}$ или $S$ является слоем расслоения $\varphi$.

Таким образом, множество $\mathbb{L} \mathbb{C} S(X, \lambda D)$ не содержит поверхностей.

Применяя теорему 2.27 к лог-паре $(Y, \lambda \gamma(D))$ и расслоению $\psi$, мы сразу получаем, что $\operatorname{LCS}(X, \lambda D) \subsetneq E_{2} \cup \bar{L}$, где $\mathbb{P}^{1} \cong \bar{L} \subset X-$ кривая такая, что $\gamma(\bar{L})$ является слоем расслоения на коники $\psi$.

Допустим, что $\bar{L} \not \subset E_{1}$ и $\bar{L} \subset \operatorname{LCS}(X, \lambda D)$. Тогда

$$
\alpha \circ \gamma(\bar{L}) \subseteq \operatorname{LCS}(Q, \lambda \alpha \circ \gamma(D)) \subseteq \alpha \circ \gamma(\bar{L}) \cup C \cup L,
$$

что невозможно по лемме 2.10. Таким образом, по теореме 2.7 имеются лишь следующие возможности: либо $\operatorname{LCS}(X, \lambda D) \subsetneq E_{2} ;$ либо $\operatorname{LCS}(X, \lambda D) \subseteq \bar{L}$ и $\bar{L} \subset E_{1}$.

Можно считать, что $\bar{L} \subset E_{1}$. При этом $E_{1} \cong \mathbb{F}_{1}$. Следовательно, $\bar{L} \cdot \bar{L}=-1$ на поверхности $E_{1}$.

Применяя лемму 2.28 к лог-паре $\left(\mathbb{P}^{1} \times \mathbb{P}^{2}, \lambda \omega(D)\right)$, получаем, что

$$
\operatorname{LCS}(X, \lambda D) \subset F
$$

так как имеет место эквивалентность $\omega(D) \sim_{\mathbb{Q}}-K_{\mathbb{P}^{1} \times \mathbb{P}^{2}}$ и $\lambda<1 / 2$. Применяя лемму 2.25 к лог-паре $(V, \lambda \beta(D))$ и расслоению $\eta$, получаем, что $\operatorname{LCS}(X, \lambda D) \subsetneq$ $E_{1} \cup S_{\varphi}$, где $S_{\varphi}-$ особый слой расслоения $\varphi$, так как $\operatorname{lct}\left(\mathbb{P}^{1} \times \mathbb{P}^{1}\right)=1 / 2($ см. пример 1.10).

Заметим, что $F \cap \bar{L}=\varnothing$ и $\left|F \cap \bar{S}_{\varphi} \cap E_{2}\right|<+\infty$. Таким образом, найдется точка $P \in E_{2}$ такая, что $\operatorname{LCS}(X, \lambda D)=P \in E_{2}$ по теореме 2.7. При этом $\beta\left(E_{1}\right) \cap \beta(P)=\varnothing$. Следовательно, по теореме 2.7 имеем $\operatorname{LCS}(V, \lambda \beta(D))=\beta(P)$.

Пусть $\widetilde{H}_{1} \subset V \supset \widetilde{H}_{2}$ - собственные прообразы дивизоров $H_{1} \subset Q \supset H_{2}$ соответственно. Тогда $-K_{V} \sim \widetilde{H}_{2}+2 \widetilde{H}_{1} \sim_{\mathbb{Q}} \beta(D)$. Из-за общности в выборе $H_{1}$ и $H_{2}$ имеем

$$
\operatorname{LCS}\left(V, \lambda \beta(D)+\frac{1}{2}\left(\widetilde{H}_{2}+2 \widetilde{H}_{1}\right)\right)=\beta(P) \cup \widetilde{H}_{1},
$$

что невозможно по теореме 2.7 , так как $\lambda<1 / 2$. 
Лемма 8.16. Пусть $I(X)=3.16$. Тогда $\operatorname{lct}(X)=1 / 2$.

ДокАЗАТЕЛЬСтво. Пусть $\mathbb{P}^{1} \cong C \subset \mathbb{P}^{3}$ - скрученная кубика, $O \in C$ - произвольная точка. Многообразие $X$ включается в коммутативную диаграмму

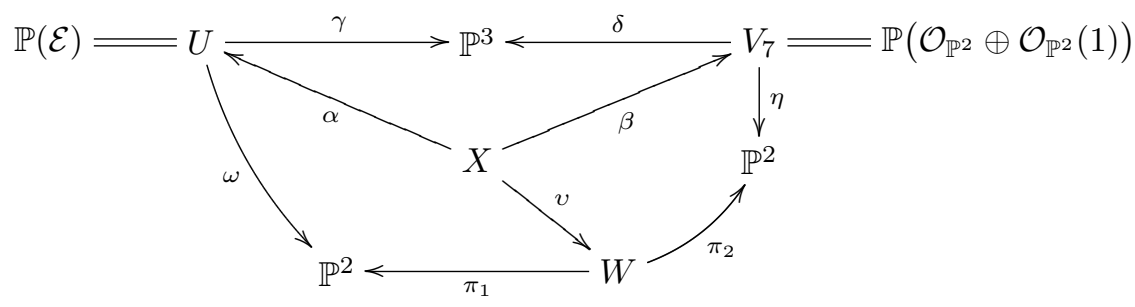

где $\mathscr{E}$ - стабильное векторное расслоение ранга 2 на $\mathbb{P}^{2}$ (см. доказательство леммы 7.13). В диаграмме использованы следующие обозначения: $\delta$ - раздутие точки $O$; морфизм $\gamma$ стягивает дивизор $G \subset U$ на кривую $C \subset \mathbb{P}^{3} ;$ морфизм $\alpha$ стягивает дивизор $E \cong \mathbb{F}_{1}$ на слой отображения $\gamma$ над точкой $O \in \mathbb{P}^{3} ;$ морфизм $\beta$ - раздутие собственного прообраза кривой $C$; многообразие $W$ - неособый дивизор бистепени $(1,1)$ на $\mathbb{P}^{2} \times \mathbb{P}^{2} ;$ морфизмы $\pi_{1}$ и $\pi_{2}$ - естественные проекции; морфизмы $\omega$ и $\eta$ - естественные $\mathbb{P}^{1}$-расслоения; морфизм $v$ стягивает дивизор $F \subset X$ на кривую $\mathbb{P}^{1} \cong Z \subset W$ такую, что $\omega \circ \alpha(E)=\pi_{1}(Z)$ и $\eta \circ \beta(G)=\pi_{2}(Z)$.

Выберем общие дивизоры $H_{1} \in\left|(\omega \circ \alpha)^{*}\left(\mathscr{O}_{\mathbb{P}^{2}}(1)\right)\right|$ и $H_{2} \in\left|(\eta \circ \beta)^{*}\left(\mathscr{O}_{\mathbb{P}^{2}}(1)\right)\right|$. Тогда $-K_{X} \sim H_{1}+2 H_{2}$, откуда следует, что $\operatorname{lct}(X) \leqslant 1 / 2$.

Предположим, что $\operatorname{lct}(X)<1 / 2$. Тогда найдется эффективный $\mathbb{Q}$-дивизор $D \sim_{\mathbb{Q}}-K_{X}$ такой, что лог-пара $(X, \lambda D)$ не лог-канонична при некотором $\lambda<1 / 2$. Заметим, что $\operatorname{LCS}(X, \lambda D) \subseteq E \cap F$, так как $\operatorname{lct}(U)=1 / 2$ по лемме 7.11 и $\operatorname{lct}(W)=1 / 2$ по теореме 6.1 .

Применяя лемму 2.12 к лог-паре $\left(V_{7}, \lambda \beta(D)\right)$, получаем, что $\operatorname{LCS}(X, \lambda D)=$ $E \cap F \cap G$, причем $|E \cap F \cap G|=1$. Таким образом,

$$
\operatorname{LCS}\left(X, \lambda D+H_{2}\right)=\operatorname{LCS}(X, \lambda D) \cup H_{2},
$$

где $H_{2} \cap \operatorname{LCS}(X, \lambda D)=\varnothing$. Но дивизор

$$
-\left(K_{X}+\lambda D+H_{2}\right) \sim_{\mathbb{Q}}\left(\lambda-\frac{1}{2}\right) K_{X}+\frac{1}{2} H_{1}
$$

обилен, что невозможно по теореме 2.7.

Лемма 8.17. Пусть $\rightrightarrows(X)=3.17$. Тогда $\operatorname{lct}(X)=1 / 2$.

ДокАЗАТЕЛЬСтво. Многообразие $X$ является дивизором тристепени $(1,1,1)$ на $\mathbb{P}^{1} \times \mathbb{P}^{1} \times \mathbb{P}^{2}$. Выберем общие дивизоры $H_{1} \in\left|\pi_{1}^{*}\left(\mathscr{O}_{\mathbb{P}^{1}}(1)\right)\right|, H_{2} \in\left|\pi_{2}^{*}\left(\mathscr{O}_{\mathbb{P}^{1}}(1)\right)\right|$, $H_{3} \in\left|\pi_{3}^{*}\left(\mathscr{O}_{\mathbb{P}^{2}}(1)\right)\right|$, где $\pi_{i}-$ проекция многообразия $X$ на $i$-й сомножитель в $\mathbb{P}^{1} \times \mathbb{P}^{1} \times \mathbb{P}^{2}$. Тогда $-K_{X} \sim H_{1}+H_{2}+2 H_{3}$, откуда следует $\operatorname{lct}(X) \leqslant 1 / 2$. 
Многообразие $X$ включается в коммутативную диаграмму

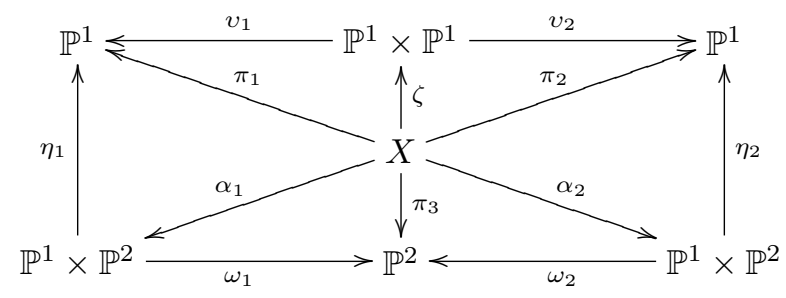

где $\omega_{i}, \eta_{i}$ и $v_{i}$ - естественные проекции, $\zeta$ является $\mathbb{P}^{1}$-расслоением, а $\alpha_{i}-$ стягивание дивизора $E_{i} \subset X$ на неособую кривую $C_{i} \subset \mathbb{P}^{1} \times \mathbb{P}^{2}$, причем $\omega_{1}\left(C_{1}\right)=$ $\omega_{2}\left(C_{2}\right)-($ неприводимая) коника.

Заметим, что $E_{2} \sim H_{1}+H_{3}-H_{2}$ и $E_{1} \sim H_{2}+H_{3}-H_{1}$.

Предположим, что $\operatorname{lct}(X)<1 / 2$. Тогда найдется эффективный $\mathbb{Q}$-дивизор $D \sim_{\mathbb{Q}}-K_{X}$ такой, что лог-пара $(X, \lambda D)$ не является лог-канонической при некотором $\lambda<1 / 2$.

Предположим, что множество $\mathbb{L} \mathbb{C} S(X, \lambda D)$ содержит (неприводимую) поверхность $S \subset X$. Пусть $D=\mu S+\Omega$, где $\mu \geqslant 1 / \lambda$ и $\Omega$ - эффективный $\mathbb{Q}$-дивизор такой, что $S \not \subset \operatorname{Supp}(\Omega)$. Тогда

$$
2=D \cdot \Gamma=\mu S \cdot \Gamma+\Omega \cdot \Gamma \geqslant \mu S \cdot \Gamma
$$

где $\Gamma \cong \mathbb{P}^{1}$ - общий слой расслоения $\zeta$. Таким образом, $S \cdot \Gamma=0$, откуда следует $E_{2} \neq S \neq E_{1}$. Кроме того,

$$
2=D \cdot \Delta=\mu S \cdot \Delta+\Omega \cdot \Delta \geqslant \mu S \cdot \Delta,
$$

где $\Delta \cong \mathbb{P}^{1}$ - общий слой расслоения $\pi_{2}$. Таким образом, $S \cdot \Delta=0$, откуда сразу следует, что $S \in\left|\pi_{3}^{*}\left(\mathscr{O}_{\mathbb{P}^{2}}(m)\right)\right|$ при некотором $m \in \mathbb{Z}_{>0}$, так как $E_{2} \neq S \neq E_{1}$ и $S$ неприводима. В частности, $0=S \cdot \Gamma=m \neq 0$, противоречие. Следовательно, множество $\mathbb{L} \mathbb{C} S(X, \lambda D)$ не содержит поверхностей.

Применяя теорему 2.27 к расслоению $\zeta$ и используя теорему 2.7, мы сразу видим, что $\operatorname{LCS}(X, \lambda D)=F$, где $F$ - некоторый слой $\mathbb{P}^{1}$-расслоения $\zeta$. Применяя теорему 2.27 к расслоению на коники $\pi_{3}$, получаем, что всякий слой расслоения $\pi_{3}$, пересекающий $F$, должен быть приводим. Следовательно,

$$
\pi_{3}(F) \subset \omega_{1}\left(C_{1}\right)=\omega_{2}\left(C_{2}\right)
$$

что невозможно, так как $\pi_{3}(F)$ - прямая, а $\omega_{1}\left(C_{1}\right)=\omega_{2}\left(C_{2}\right)$ - неприводимая коника.

Лемма 8.18. Пусть $I(X)=3.18$. Тогда $\operatorname{lct}(X)=1 / 3$.

ДоказАтельство. Пусть $Q \subset \mathbb{P}^{4}-$ неособая квадрика, $C \subset Q-$ (неприводимая) коника, $O \in C$ - точка на ней. Тогда $X$ включается в коммутативную 
диаграмму

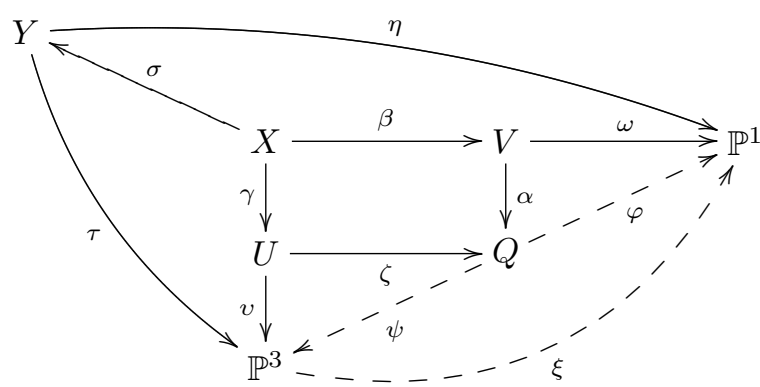

где $\zeta$ - раздутие точки $O$, морфизмы $\alpha$ и $\gamma$ - раздутия коники $C$ и ее собственного прообраза соответственно, $\beta$ - раздутие слоя морфизма $\alpha$ над точкой $O$, отображение $\psi$ - проекция из точки $O$, отображение $\varphi$ индуцировано проекцией из двумерного линейного подпространства в $\mathbb{P}^{4}$, содержащего конику $C$, морфизм $\tau$ - раздутие прямой $\psi(C)$, морфизм $v$ - раздутие (неприводимой) коники $Z \subset \mathbb{P}^{3}$ такой, что $\psi(C) \cap Z \neq \varnothing$, и $Z$ и $\psi(C)$ не содержатся в одной плоскости, морфизм $\sigma$ - раздутие собственного прообраза коники $Z$, отображение $\xi$ - проекция из прямой $\psi(C)$, морфизм $\eta$ является $\mathbb{P}^{1}$-расслоением, а $\omega-$ расслоение на квадрики.

Пусть $\bar{H}$ - общий слой отображения $\omega \circ \beta$. Тогда $\bar{H}$ является поверхностью дель Пеццо степени $K_{\bar{H}}^{2}=7$ и $-K_{X} \sim 3 \bar{H}+2 E+G$, где $G$ и $E-$ исключительные дивизоры раздутий $\beta$ и $\gamma$ соответственно. В частности, $\operatorname{lct}(X) \leqslant 1 / 3$.

Предположим, что $\operatorname{lct}(X)<1 / 3$. Тогда найдется эффективный $\mathbb{Q}$-дивизор $D \sim_{\mathbb{Q}}-K_{X}$ такой, что лог-пара $(X, \lambda D)$ не лог-канонична при некотором $\lambda<1 / 3$. При этом $\operatorname{LCS}(X, \lambda D) \subseteq G$, так как $\operatorname{lct}(V)=1 / 3$ по лемме 7.15 и $\beta(D) \sim_{\mathbb{Q}}-K_{V}$.

Применяя лемму 2.25 к расслоению на поверхности дель Пеццо $\omega \circ \beta$, с учетом теоремы 2.7 получаем, что существует такой особый слой $S$ расслоения $\omega \circ \beta$, что $\operatorname{LCS}(X, \lambda D) \subseteq G \cap S$, так как выполнено равенство $\operatorname{lct}(\bar{H})=1 / 3$ (см. пример 1.10).

Пусть $P \in G \cap S$ - произвольная точка множества $\operatorname{LCS}(X, \lambda D)$. Положим $D=\mu S+\Omega$, где $\Omega$ - эффективный $\mathbb{Q}$-дивизор такой, что $S \not \subset \operatorname{Supp}(\Omega)$. Тогда $P \in \operatorname{LCS}\left(S,\left.\lambda \Omega\right|_{S}\right)$ по теореме 2.19 .

Отождествим поверхность $\beta(S)$ с квадратичным конусом в $\mathbb{P}^{3}$. Заметим, что $G \cap S$ - исключительная кривая на $S$, т. е. существует единственная образующая конуса $\beta(S)$, пересекающая кривую $\beta(G)$. Пусть $L \subset S$ - собственный прообраз этой образующей. Тогда $L \cap G \neq \varnothing$ (более того, $|L \cap G|=1$ ), в то время как $L \cap E=\varnothing$. Следовательно, $P=L \cap G$ по лемме 4.10. В частности, $\operatorname{LCS}(X, \lambda D)=P$. Следовательно,

$$
\bar{H} \cup P \subseteq \operatorname{LCS}\left(X, \lambda D+\bar{H}+\frac{2}{3} E\right) \subseteq \bar{H} \cup P \cup E,
$$

так как $\bar{H}$ - достаточно общий слой расслоения $\omega \circ \beta$. Следовательно, множество лог-канонических особенностей $\operatorname{LCS}\left(X, \lambda D+\bar{H}+\frac{2}{3} E\right)$ должно быть 
несвязным, так как $P \notin \bar{H}$ и $P \notin E$. Но дивизор

$$
-\left(K_{X}+\lambda D+\bar{H}+\frac{2}{3} E\right) \sim_{\mathbb{Q}} \bar{H}+\frac{2}{3}(E+G)+\left(\lambda-\frac{1}{3}\right) K_{X}
$$

обилен, что невозможно по теореме 2.7.

Из доказательства леммы 8.18 вытекает следующее утверждение.

СлЕДСТвиЕ 8.19. Пусть $\Xi(X)=4.4$ или $\Xi(X)=5.1$. Тогда $\operatorname{lct}(X)=1 / 3$.

ЛЕмма 8.20. Пусть $I(X)=3.19$. Тогда $\operatorname{lct}(X)=1 / 3$.

ДокАЗАТЕЛьство. Пусть $Q \subset \mathbb{P}^{4}$ - неособая квадрика, а $L \subset \mathbb{P}^{4}$ - такая прямая, что $L \cap Q=P_{1} \cup P_{2}$, где $P_{1}$ и $P_{2}$ - различные точки. Пусть $\eta: Q \rightarrow \mathbb{P}^{2}-$ проекция из прямой $L$. Многообразие $X$ включается в коммутативную диаграмму

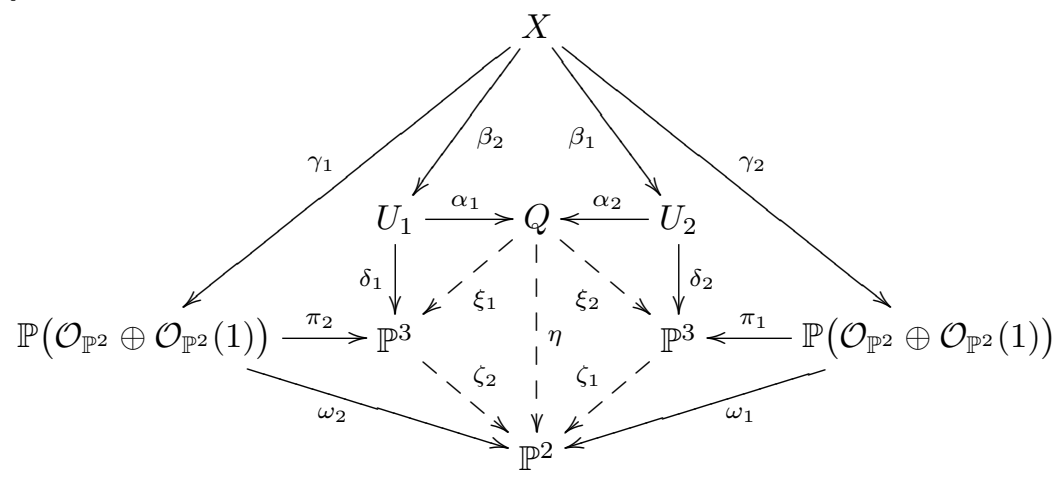

где $\alpha_{i}$ - раздутие точки $P_{i}$, морфизм $\beta_{i}$ стягивает поверхность $\mathbb{P}^{2} \cong E_{i} \subset X$ в точку, лежащую над точкой $P_{i} \in Q$, отображение $\xi_{i}-$ проекция из точки $P_{i}$, отображение $\zeta_{i}-$ проекция из образа точки $P_{i}$, морфизм $\delta_{i}$ стягивает поверхность $\mathbb{F}_{2} \cong G_{i} \subset U_{i}$ на конику $C_{i} \subset \mathbb{P}^{3}$, морфизм $\pi_{i}$ является раздутием образа точки $P_{i}$, морфизм $\gamma_{i}$ стягивает собственный прообраз дивизора $G_{i}$ на собственный прообраз кривой $C_{i}$, а $\omega_{i}$ - естественная проекция.

Отображение $\gamma_{1} \circ \gamma_{2}^{-1}$ является элементарным преобразованием расслоения на коники (см. [57]), и поверхности $\delta_{1} \circ \beta_{2}\left(E_{1}\right) \subset \mathbb{P}^{3} \supset \delta_{2} \circ \beta_{1}\left(E_{2}\right)$ являются плоскостями, содержащими коники $C_{1}$ и $C_{2}$ соответственно.

Пусть $H$ - общее гиперплоское сечение квадрики $Q$ такое, что $P_{1} \in H \ni P_{2}$. Тогда $-K_{X} \sim 3 \bar{H}+E_{1}+E_{2}$, где $\bar{H}$ - собственный прообраз дивизора $H$ на многообразии $X$. В частности, $\operatorname{lct}(X) \leqslant 1 / 3$.

Предположим, что $\operatorname{lct}(X)<1 / 3$. Тогда найдется эффективный $\mathbb{Q}$-дивизор $D \sim_{\mathbb{Q}}-K_{X}$ такой, что особенности лог-пары $(X, \lambda D)$ не являются лог-каноническими при некотором $\lambda<1 / 3$. Тогда $\operatorname{LCS}(X, \lambda D) \subseteq E_{1} \cup E_{2}$, так как $\operatorname{lct}(Q)=1 / 3$. По теореме 2.7 можно предполагать, что $\operatorname{LCS}(X, \lambda D) \subseteq E_{1}$.

Пусть $\bar{G}_{2} \subset X$ - собственный прообраз дивизора $G_{2}$. Тогда $\bar{G}_{2} \cap E_{1}=\varnothing$, так как $\alpha_{2}\left(G_{2}\right) \subset Q$ - квадратичный конус с вершиной в точке $P_{2}$, а прямая $L$ не содержится в $Q$. Следовательно,

$$
\varnothing \neq \operatorname{LCS}\left(\mathbb{P}\left(\mathscr{O}_{\mathbb{P}^{2}} \oplus \mathscr{O}_{\mathbb{P}^{2}}(1)\right), \lambda \gamma_{2}(D)\right) \subseteq \gamma_{2}\left(E_{1}\right),
$$


где $\gamma_{2}\left(E_{1}\right)$ является сечением расслоения $\omega_{1}$. Применяя теорему 2.27 к расслоению $\omega_{1}$, приходим к противоречию.

Лемма 8.21. Пусть $I(X)=3.20$. Тогда $\operatorname{lct}(X)=1 / 3$.

ДокАЗАтельство. Пусть $Q \subset \mathbb{P}^{4}$ - неособая квадрика, а $W$ является неособым дивизором бистепени $(1,1)$ на $\mathbb{P}^{2} \times \mathbb{P}^{2}$. Пусть $L_{1} \subset Q \supset L_{2}$ - непересекающиеся прямые. Многообразие $X$ включается в коммутативную диаграмму

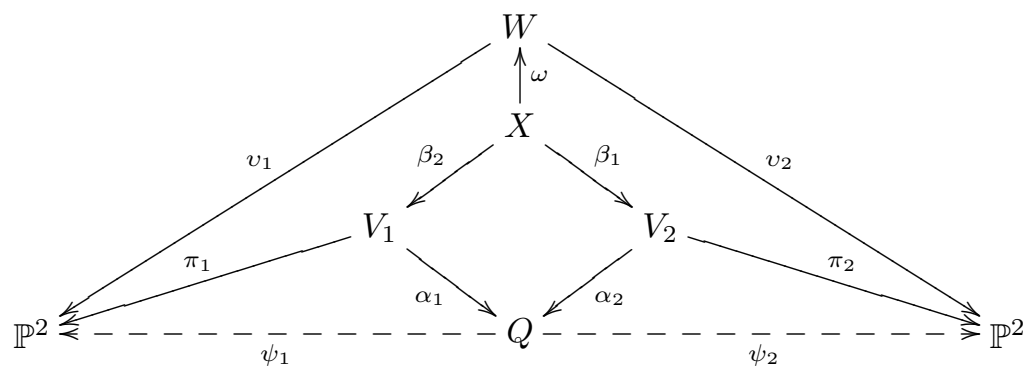

где $\alpha_{i}$ и $\beta_{i}$ - раздутия прямых $L_{i}$ и их собственных прообразов соответственно, $\omega$ - раздутие кривой $C \subset W$ бистепени $(1,1)$, морфизмы $v_{i}$ и $\pi_{i}$ являются естественными $\mathbb{P}^{1}$-расслоениями, а отображение $\psi_{i}$ - проекция из прямой $L_{i}$.

Пусть $\bar{H}$ - исключительный дивизор раздутия $\omega$, а $E_{i}$ - исключительный дивизор раздутия $\beta_{i}$. Тогда $-K_{X} \sim 3 \bar{H}+2 E_{1}+2 E_{2}$, так как $\alpha_{2} \circ \beta_{1}(\bar{H}) \subset Q-$ гиперплоское сечение, содержащее прямые $L_{1}$ и $L_{2}$. В частности, $\operatorname{lct}(X) \leqslant 1 / 3$.

Предположим, что $\operatorname{lct}(X)<1 / 3$. Тогда найдется эффективный $\mathbb{Q}$-дивизор $D \sim_{\mathbb{Q}}-K_{X}$ такой, что особенности лог-пары $(X, \lambda D)$ не являются лог-каноническими при некотором $\lambda<1 / 3$. Тогда $\operatorname{LCS}(X, \lambda D) \subseteq E_{1} \cap E_{2} \cap \bar{H}=\varnothing$, так как $\operatorname{lct}\left(V_{1}\right)=\operatorname{lct}\left(V_{2}\right)=1 / 3$ по лемме 7.17 и $\operatorname{lct}(W)=1 / 2$ по теореме 6.1 , противоречие.

Лемма 8.22. Пусть $I(X)=3.21$. Тогда $\operatorname{lct}(X)=1 / 3$.

ДоказАтельство. Пусть $\pi_{1}: \mathbb{P}^{1} \times \mathbb{P}^{2} \rightarrow \mathbb{P}^{1}$ и $\pi_{2}: \mathbb{P}^{1} \times \mathbb{P}^{2} \rightarrow \mathbb{P}^{2}$ - естественные проекции. Существует стягивание $\alpha: X \rightarrow \mathbb{P}^{1} \times \mathbb{P}^{2}$ поверхности $E$ на кривую $C$ такую, что $\pi_{1}^{*}\left(\mathscr{O}_{\mathbb{P}^{1}}(1)\right) \cdot C=2$ и $\pi_{2}^{*}\left(\mathscr{O}_{\mathbb{P}^{2}}(1)\right) \cdot C=1$.

Кривая $\pi_{2}(C) \subset \mathbb{P}^{2}$ - прямая. Таким образом, существует единственная поверхность $H_{2} \in\left|\pi_{2}^{*}\left(\mathscr{O}_{\mathbb{P}^{2}}(1)\right)\right|$ такая, что $C \subset H_{2}$. Пусть $H_{1}-$ слой $\mathbb{P}^{2}$-расслоения $\pi_{1}$. Тогда $-K_{X} \sim 2 \bar{H}_{1}+3 \bar{H}_{2}+2 E$, где $\bar{H}_{i} \subset X$ собственный прообраз поверхности $H_{i}$. Имеем $\operatorname{lct}(X) \leqslant 1 / 3$.

Предположим, что $\operatorname{lct}(X)<1 / 3$. Тогда найдется эффективный $\mathbb{Q}$-дивизор $D \sim_{\mathbb{Q}}-K_{X}$ такой, что лог-пара $(X, \lambda D)$ не лог-канонична при некотором $\lambda<1 / 3$. Заметим, что $\operatorname{LCS}(X, \lambda D) \subseteq E$, так как $\operatorname{lct}\left(\mathbb{P}^{1} \times \mathbb{P}^{2}\right)=1 / 3$ по лемме 2.21. Многообразие $X$ включается в коммутативную диаграмму

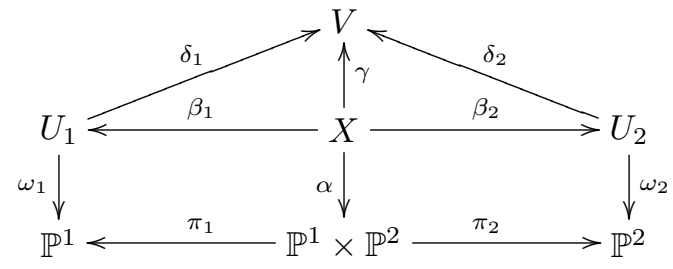


где $V$ - многообразие Фано индекса 2 с одной обыкновенной двойной точкой $O \in V$ такое, что $-K_{V}^{3}=40$, бирациональный морфизм $\beta_{i}$ - стягивание поверхности $\bar{H}_{2} \cong \mathbb{P}^{1} \times \mathbb{P}^{1}$ на неособую рациональную кривую, $\delta_{i}-$ стягивание кривой $\beta_{i}\left(\bar{H}_{2}\right)$ в точку $O \in V$, причем рациональное отображение $\delta_{2} \circ \delta_{1}^{-1}: U_{1} \rightarrow U_{2}$ является стандартным флопом в кривой $\beta_{1}\left(\bar{H}_{2}\right) \cong \mathbb{P}^{1}$, морфизм $\omega_{1}-$ расслоение с общим слоем $\mathbb{P}^{1} \times \mathbb{P}^{1}$, морфизм $\omega_{2}$ является $\mathbb{P}^{1}$-расслоением, а $\gamma$ - стягивает поверхность $\gamma\left(\bar{H}_{2}\right)$ в точку $O \in V$.

Многообразие $V$ является сечением грассманиана $\operatorname{Gr}(2,5) \subset \mathbb{P}^{9}$ линейным подпространством коразмерности 3. При этом $-K_{V} \sim 2\left(\gamma\left(\bar{H}_{1}\right)+\gamma(E)\right)$ и дивизор $\gamma\left(\bar{H}_{1}\right)+\gamma(E)$ очень обилен. Многообразие $X$ включается в коммутативную диаграмму

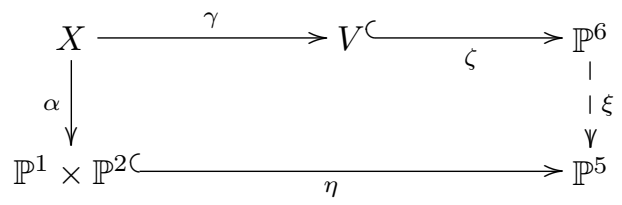

где вложение $\zeta$ задается линейной системой $\left|\gamma\left(\bar{H}_{1}\right)+\gamma(E)\right|$, отображение $\xi-$ проекция из точки $O$, а вложение $\eta$ задается линейной системой $\left|H_{1}+H_{2}\right|$.

Из [60; теорема 3.6] следует (см. также [59; теорема 3.13$])$, что $U_{2} \cong \mathbb{P}(\mathscr{E})$, где $\mathscr{E}$ - стабильное векторное расслоение ранга 2 на $\mathbb{P}^{2}$ такое, что последовательность

$$
0 \rightarrow \mathscr{O}_{\mathbb{P}_{2}} \rightarrow \mathscr{E} \otimes \mathscr{O}_{\mathbb{P}_{2}}(1) \rightarrow \mathscr{I} \otimes \mathscr{O}_{\mathbb{P}_{2}}(1) \rightarrow 0
$$

точна, $\mathscr{I}$ - пучок идеалов пары общих точек на $\mathbb{P}^{2}$. При этом $c_{1}(\mathscr{E})=-1$ и $c_{2}(\mathscr{E})=2$. Из [60; теорема 3.5$]$ следует, что

$$
U_{1} \subset \mathbb{P}\left(\mathscr{O}_{\mathbb{P}^{1}} \oplus \mathscr{O}_{\mathbb{P}^{1}}(1) \oplus \mathscr{O}_{\mathbb{P}^{1}}(1) \oplus \mathscr{O}_{\mathbb{P}^{1}}(1)\right)
$$

и $U_{1} \in|2 T-F|$, где $T$ - тавтологическое линейное расслоение на многообразии $\mathbb{P}\left(\mathscr{O}_{\mathbb{P}^{1}} \oplus \mathscr{O}_{\mathbb{P}^{1}}(1) \oplus \mathscr{O}_{\mathbb{P}^{1}}(1) \oplus \mathscr{O}_{\mathbb{P}^{1}}(1)\right)$, а $F-$ слой проекции $\mathbb{P}\left(\mathscr{O}_{\mathbb{P}^{1}} \oplus \mathscr{O}_{\mathbb{P}^{1}}(1) \oplus \mathscr{O}_{\mathbb{P}^{1}}(1) \oplus\right.$ $\left.\mathscr{O}_{\mathbb{P}^{1}}(1)\right) \rightarrow \mathbb{P}^{1}$.

Заметим, что так как $H_{1} \cdot C=2$, то либо $\bar{H}_{1}$ является неособой поверхностью дель Пеццо степени $K_{\bar{H}_{1}}^{2}=7$, либо $\left|H_{1} \cap C\right|=1$. Применяя лемму 2.25 к морфизму $\omega_{1} \circ \beta_{1}$ и поверхности $\bar{H}_{1}$, мы видим, что либо $\left|H_{1} \cap C\right|=1$, либо $H_{1} \cap \operatorname{LCS}(X, \lambda D)=\varnothing$, так как $\operatorname{lct}\left(\bar{H}_{1}\right)=1 / 3$ в случае, если поверхность $\bar{H}_{1}$ неособа. Таким образом, существует слой $L$ проекции $E \rightarrow C$ такой, что $\operatorname{LCS}(X, \lambda D) \subseteq L$ по теореме 2.7. Положим $\bar{C}=\bar{H}_{2} \cap E$ и $P=L \cap \bar{C}$. Применяя теорему 2.27 к расслоению $\omega_{2}$ и лог-паре $\left(U_{2}, \lambda \beta_{2}(D)\right)$, по теореме 2.7 получаем, что $\operatorname{LCS}(X, \lambda D)=P$ или $\operatorname{LCS}(X, \lambda D)=L$.

Допустим, что $\operatorname{LCS}(X, \lambda D)=L$. Тогда

$$
\operatorname{LCS}(V, \lambda \gamma(D))=\gamma(L)
$$

где $\gamma(L) \subset V \subset \mathbb{P}^{6}-$ прямая, так как $-K_{V} \cdot \gamma(L)=2$ и $-K_{V} \sim_{\mathbb{Q}} \gamma(D)$. Кроме того, $\operatorname{Sing}(V)=O \in \gamma(L)$.

Пусть $S \subset V$ - общее гиперплоское сечение $V \subset \mathbb{P}^{6}$ такое, что $\gamma(L) \subset S$. Тогда поверхность $S$ является поверхностью дель Пеццо степени $K_{S}^{2}=5$, точка $O$ является обыкновенной двойной особенностью поверхности $S$, поверхность $S$ 
неособа вне точки $O \in \gamma(L)$, выполнено соотношение $\left.K_{S} \sim \mathscr{O}_{\mathbb{P} 6}(1)\right|_{S}$, откуда следует, что на поверхности $S$ лежит конечное число прямых, пересекающих прямую $\gamma(L)$.

Пусть $H \subset V$ - общее гиперплоское сечение $V \subset \mathbb{P}^{6}$. Положим $Q=\gamma(L) \cap H$. Тогда $\operatorname{LCS}\left(H,\left.\lambda \gamma(D)\right|_{H}\right)=Q$ по замечанию 2.3 , что противоречит лемме 4.2 , так как $\lambda<1 / 3$.

Таким образом, $\operatorname{LCS}(X, \lambda D)=P \in \bar{C}$. Пусть $F_{1}$ - общий слой отображения $\pi_{1}$. Тогда

$$
F_{1} \cap C=P_{1} \cup P_{2} \not \supset \alpha(P),
$$

где $P_{1}$ и $P_{2}$ - различные точки. При этом $P_{1} \cup P_{2} \subset H_{2} \cap F_{1}$, так как $C \subset H_{2}$. Пусть $Z$ - общая прямая на $F_{1} \cong \mathbb{P}^{2}$, проходящая через точку $P_{1}$. Тогда существует дивизор $F_{2} \in\left|\pi_{2}^{*}\left(\mathscr{O}_{\mathbb{P}^{2}}(1)\right)\right|$ такой, что $Z \subset F_{2}$. Пусть $\bar{F}_{1} \subset X \supset \bar{F}_{2}-$ собственные прообразы дивизоров $F_{1}$ и $F_{2}$ соответственно. Тогда $P \notin \bar{F}_{1} \cup \bar{F}_{2}$.

Пусть $\bar{Z} \subset X$ - собственный прообраз кривой $Z$. Тогда $-K_{X} \cdot \bar{Z}=2$ и $\bar{Z} \subset$ $\bar{F}_{1} \cap \bar{F}_{2}$, причем $\bar{Z} \cap \bar{H}_{2}=\varnothing$. Таким образом, кривая $\gamma(\bar{Z})$ является прямой на $V \subset \mathbb{P}^{6}$ такой, что $\operatorname{Sing}(V)=O \notin \gamma(\bar{Z})$.

Пусть $T$ - общее гиперплоское сечение многообразия $V \subset \mathbb{P}^{6}$ такое, что $\gamma(\bar{Z}) \subset T$. Тогда

$$
\bar{T} \sim 2 \bar{H}_{2}+\bar{H}_{1}+E \sim 2 \bar{H}_{2}+\bar{F}_{1}+E \sim 2 \bar{F}_{2}+\bar{F}_{1}-E,
$$

где $\bar{T}$ - собственный прообраз поверхности $T$ на многообразии $X$. Таким образом,

$$
\bar{F}_{1}+\bar{F}_{2}+\bar{T} \sim 3 \bar{F}_{2}+2 \bar{F}_{1}-E \sim 2 \bar{H}_{2}+2 \bar{H}_{1}+2 E \sim-K_{X},
$$

и, применяя теорему 2.7, мы видим, что множество

$$
P \cup \bar{Z}=\operatorname{LCS}\left(X, \lambda D+\frac{2}{3}\left(\bar{F}_{1}+\bar{F}_{2}+\bar{T}\right)\right)
$$

должно быть связным. Однако $P \notin \bar{Z}$, противоречие.

Лемма 8.23. Пусть $I(X)=3.22$. Тогда $\operatorname{lct}(X)=1 / 3$.

ДокАЗАтельство. Пусть $\pi_{1}: \mathbb{P}^{1} \times \mathbb{P}^{2} \rightarrow \mathbb{P}^{1}$ и $\pi_{2}: \mathbb{P}^{1} \times \mathbb{P}^{2} \rightarrow \mathbb{P}^{2}$ - естественные проекции. Существует стягивание $\alpha: X \rightarrow \mathbb{P}^{1} \times \mathbb{P}^{2}$ поверхности $E$ на кривую $C$, содержащуюся в слое $H_{1}$ проекции $\pi_{1}$, такую, что кривая $\pi_{2}(C)$ является коникой на $\mathbb{P}^{2}$.

Заметим, что $E \cong \mathbb{F}_{2}$. Пусть $H_{2}$ - общая поверхность в линейной системе $\left|\pi_{2}^{*}\left(\mathscr{O}_{\mathbb{P}^{2}}(1)\right)\right|$. Тогда $-K_{X} \sim 2 \bar{H}_{1}+3 \bar{H}_{2}+E$, где $\bar{H}_{i} \subset X-$ собственный прообраз дивизора $H_{i}$. Следовательно, $\operatorname{lct}(X) \leqslant 1 / 3$.

Предположим, что $\operatorname{lct}(X)<1 / 3$. Тогда найдется эффективный $\mathbb{Q}$-дивизор $D \sim_{\mathbb{Q}}-K_{X}$ такой, что лог-пара $(X, \lambda D)$ не лог-канонична при некотором $\lambda<1 / 3$. При этом $\operatorname{LCS}(X, \lambda D) \subseteq E$, так как $\operatorname{lct}\left(\mathbb{P}^{1} \times \mathbb{P}^{2}\right)=1 / 3$ по лемме 2.21 .

Пусть $Q-($ единственный $)$ дивизор в линейной системе $\left|\pi_{2}^{*}\left(\mathscr{O}_{\mathbb{P}^{2}}(2)\right)\right|$, содержащий кривую $C$, а $\bar{Q} \subset X$ - собственный прообраз дивизора $Q$. Тогда 
$\bar{Q} \cap \bar{H}_{1}=\varnothing$ и многообразие $X$ включается в коммутативную диаграмму

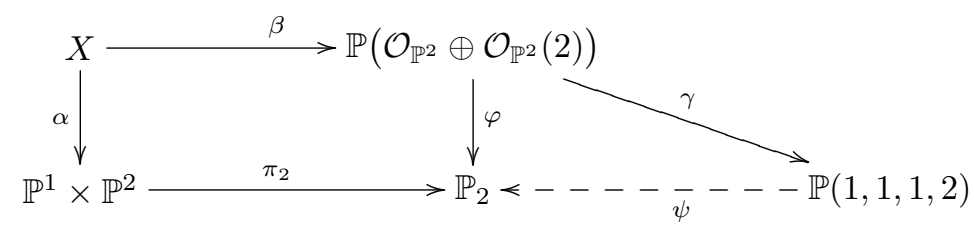

где $\beta$ - стягивание поверхности $\bar{Q}$ на кривую, $\gamma$ - стягивание поверхности $\beta\left(\bar{H}_{1}\right)$ в точку, морфизм $\varphi$ - естественное $\mathbb{P}^{1}$-расслоение, а отображение $\psi$ - естественная проекция. При этом

$$
\gamma \circ \beta(D) \sim_{\mathbb{Q}} \frac{5 \gamma \circ \beta(E)}{2} \sim_{\mathbb{Q}}-K_{\mathbb{P}(1,1,1,2)} \sim_{\mathbb{Q}} \mathscr{O}_{\mathbb{P}(1,1,1,2)}(5),
$$

откуда следует, что $E \nsubseteq \operatorname{LCS}(X, \lambda D)$, так как $\lambda<1 / 3$.

Применяя теорему 2.27 к расслоению $\varphi$, получаем, что существует слой $F$ проекции $E \rightarrow C$ такой, что $\operatorname{LCS}(X, \lambda D) \subseteq(E \cap \bar{Q}) \cup F$, при этом, возможно, $\operatorname{LCS}(X, \lambda D) \subset E \cap \bar{Q}$.

Допустим, что $\operatorname{LCS}(X, \lambda D) \subset E \cap \bar{Q}$. Пусть $M \subset \mathbb{P}^{1} \times \mathbb{P}^{2}-$ общий дивизор в линейной системе $\left|H_{1}+H_{2}\right|$, а $\bar{M} \subset X$ - собственный прообраз дивизора $M$. Тогда $\bar{M} \cap \bar{H}_{1}=L$, где $L-$ прямая на $\bar{H}_{1} \cong \mathbb{P}^{2}$. Пусть $R-$ (единственный) дивизор в линейной системе $\left|\pi_{2}^{*}\left(\mathscr{O}_{\mathbb{P}^{2}}(1)\right)\right|$, проходящий через кривую $\alpha(L)$, а $\bar{R}-$ собственный прообраз дивизора $R$ на многообразии $X$. Тогда

$\operatorname{LCS}(X, \lambda D) \cup L \subseteq \operatorname{LCS}\left(X, \lambda D+\frac{2}{3}\left(\bar{M}+\bar{H}_{1}+\bar{R}+\bar{H}_{2}\right)\right) \subseteq \operatorname{LCS}(X, \lambda D) \cup L \cup \bar{H}_{1}$,

но $L \cap E \cap \bar{Q}=\bar{Q} \cap \bar{H}_{1}=\varnothing$ и $-K_{X} \sim \bar{M}+\bar{H}_{1}+\bar{R}+\bar{H}_{2}$, что противоречит теореме 2.7 .

Таким образом, $F \subseteq \operatorname{LCS}(X, \lambda D)$. Положим $\breve{F}=\gamma \circ \beta(F)$ и $\breve{D}=\gamma \circ \beta(D)$. Тогда

$$
\breve{F} \subseteq \operatorname{LCS}(\mathbb{P}(1,1,1,2), \lambda \breve{D}) \subseteq \breve{C} \cup \breve{F}
$$

где $\breve{C}=\gamma \circ \beta(\bar{Q}) \subset \mathbb{P}(1,1,1,2)$ - кривая такая, что $\psi(\breve{C})=\pi_{2}(C)$.

Пусть $S$ - общий дивизор в линейной системе $\left|\mathscr{O}_{\mathbb{P}(1,1,1,2)}(2)\right|$. Тогда $S \cong \mathbb{P}^{2}$ и

$$
\breve{F} \cap S \subseteq \operatorname{LCS}\left(S,\left.\lambda \breve{D}\right|_{S}\right) \subseteq(\breve{C} \cup \breve{F}) \cap S
$$

но $\left.3 D\right|_{S} \sim_{\mathbb{Q}}-5 K_{S}$, что невозможно по лемме 2.8 .

Лемма 8.24. Пусть $I(X)=3.23$. Тогда $\operatorname{lct}(X)=1 / 4$.

ДокАЗАтельство. Пусть $O \in \mathbb{P}^{3}$ - точка, $C \subset \mathbb{P}^{3}-$ коника, проходящая через точку $O \in C$; пусть $\Pi \subset \mathbb{P}^{3}-$ (единственная) плоскость, содержащая 
конику $C$, а $Q \subset \mathbb{P}^{4}$ - неособая квадрика. Многообразие $X$ включается в коммутативную диаграмму

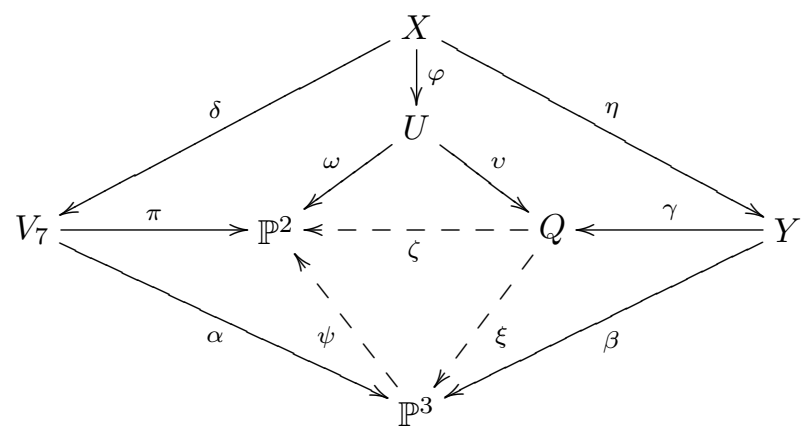

В диаграмме использованы следующие обозначения: морфизм $\alpha$ - раздутие точки $O$ с исключительным дивизором $E$; морфизм $\pi$ - естественное $\mathbb{P}^{1}$-расслоение; морфизмы $\beta$ и $\delta$ - раздутия коники $C$ и ее собственного прообраза соответственно; морфизм $\gamma$ стягивает собственный прообраз плоскости П в точку; морфизм $\varphi$ стягивает собственный прообраз плоскости П на кривую; морфизм $\eta$ стягивает собственный прообраз дивизора $E$ на такую кривую $L \subset Y$, что $\gamma(\Pi) \in \gamma(L) \subset Q \subset \mathbb{P}^{4}$ и $\gamma(L)$ - прямая в $\mathbb{P}^{4}$; морфизм $\omega-$ естественное $\mathbb{P}^{1}$-расслоение; морфизм $v$ - раздутие прямой $\gamma(L)$; отображения $\psi, \xi$ и $\zeta$ являются проекциями из $O, \gamma(\Pi)$ и $\gamma(L)$ соответственно. Заметим, что поверхность $E$ является сечением расслоения $\pi$.

Пусть $\bar{\Pi} \subset X$ - собственный прообраз плоскости $\Pi \subset \mathbb{P}^{3}$. Тогда $\operatorname{lct}(X) \leqslant 1 / 4$, так как выполнено соотношение $-K_{X} \sim 4 \bar{\Pi}+2 \bar{E}+3 G$, где $\bar{E}$ и $G$ - исключительные дивизоры стягиваний $\eta$ и $\delta$ соответственно.

Предположим, что $\operatorname{lct}(X)<1 / 4$. Тогда найдется эффективный $\mathbb{Q}$-дивизор $D \sim_{\mathbb{Q}}-K_{X}$ такой, что лог-пара $(X, \lambda D)$ не лог-канонична при некотором $\lambda<1 / 4$. Заметим, что

$$
\varnothing \neq \operatorname{LCS}(X, \lambda D) \subseteq \bar{E} \cap \bar{\Pi} \cap G,
$$

так как $\operatorname{lct}\left(V_{7}\right)=1 / 4$ по теореме $6.1, \operatorname{lct}(Y)=1 / 4$ по лемме 7.16 и $\operatorname{lct}(U)=1 / 3$ по лемме 7.17.

Пусть $R \subset \mathbb{P}^{3}$ - общий конус над коникой $C$ с вершиной $P \in \mathbb{P}^{3}$, пусть $H_{1} \subset \mathbb{P}^{3}$ - общая плоскость, проходящая через точки $O$ и $P$, и пусть $H_{2} \subset \mathbb{P}^{3}-$ общая плоскость, проходящая через точку $P$. Тогда

$$
\bar{R} \sim(\alpha \circ \delta)^{*}(R)-\bar{E}-G, \quad \bar{H}_{1} \sim(\alpha \circ \delta)^{*}\left(H_{1}\right)-\bar{E}, \quad \bar{H}_{2} \sim(\alpha \circ \delta)^{*}\left(H_{2}\right),
$$

где $\bar{R}, \bar{H}_{1}, \bar{H}_{2}$ - собственные прообразы поверхностей $R, H_{1}, H_{2}$ на многообразии $X$ соответственно. При этом $-K_{X} \sim \bar{Q}+\bar{H}_{1}+\bar{H}_{2}$, и из общности в выборе $R, H_{1}$ и $H_{2}$ следует, что множество

$$
\operatorname{LCS}\left(X, \lambda D+\frac{3}{4}\left(\bar{Q}+\bar{H}_{1}+\bar{H}_{2}\right)\right)=\operatorname{LCS}(X, \lambda D) \cup P
$$

несвязно, что невозможно по теореме 2.7.

Лемма 8.25. Пусть $I(X)=3.24$. Тогда $\operatorname{lct}(X)=1 / 3$. 
ДоказАтельство. Пусть $W$ - дивизор бистепени $(1,1)$ на $\mathbb{P}^{2} \times \mathbb{P}^{2}$. Многообразие $X$ включается в коммутативную диаграмму

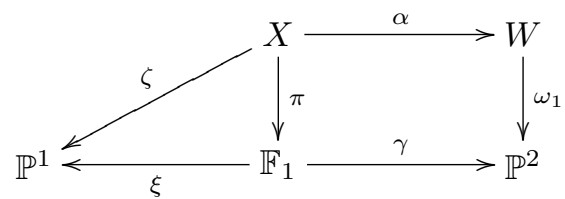

где $\omega_{1}$ - естественное $\mathbb{P}^{1}$-расслоение, $\alpha$ стягивает поверхность $E \cong \mathbb{P}^{1} \times \mathbb{P}^{1}$ на слой $L$ расслоения $\omega_{1}$, морфизм $\gamma$ - раздутие точки $\omega_{1}(L)$, морфизм $\xi$ является $\mathbb{P}^{1}$-расслоением, а морфизм $\zeta$ является $\mathbb{F}_{1}$-расслоением.

Пусть $\omega_{2}: X \rightarrow \mathbb{P}^{2}$ - естественное $\mathbb{P}^{1}$-расслоение, отличное от $\omega_{1}$. Тогда найдется дивизор $G \in\left|\omega_{2}^{*}\left(\mathscr{O}_{\mathbb{P} 2}(1)\right)\right|$ такой, что $L \subset G$, так как $\omega_{2}(L)$ - прямая на $\mathbb{P}^{2}$. Пусть $\bar{G} \subset X$ - собственный прообраз дивизора $G$. Тогда выполнена эквивалентность $-K_{X} \sim 2 F+2 \bar{G}+3 E$, где $E-$ исключительный дивизор раздутия $\alpha$, а $F$ - слой расслоения $\zeta$. Отсюда следует неравенство $\operatorname{lct}(X) \leqslant 1 / 3$.

Предположим, что $\operatorname{lct}(X)<1 / 3$. Тогда найдется эффективный $\mathbb{Q}$-дивизор $D \sim_{\mathbb{Q}}-K_{X}$ такой, что лог-пара $(X, \lambda D)$ не лог-канонична при некотором $\lambda<1 / 3$. Заметим, что $\operatorname{LCS}(X, \lambda D) \subseteq E$, так как $\operatorname{lct}(W)=1 / 2$ по теореме 6.1 . Можно считать, что $F \cap \operatorname{LCS}(X, \lambda D) \neq \varnothing$. Тогда

$$
\mathbb{F}_{1} \cong F \subseteq \operatorname{LCS}(X, \lambda D) \subseteq E \cong \mathbb{P}^{1} \times \mathbb{P}^{1}
$$

по лемме 2.25 , так как $\operatorname{lct}(F)=1 / 3$ (см. пример 1.10), противоречие.

\section{9. Многообразия с числом Пикара $\rho \geqslant 4$}

На протяжении этого раздела мы будем пользоваться предположениями и обозначениями, введенными в разделе 1.

Лемма 9.1. Пусть $I(X)=4.1$. Тогда $\operatorname{lct}(X)=1 / 2$.

ДокАЗАтЕльство. Многообразие $X$ является дивизором мультистепени $(1,1,1,1)$ на $\mathbb{P}^{1} \times \mathbb{P}^{1} \times \mathbb{P}^{1} \times \mathbb{P}^{1}$. Выберем координаты $\left[\left(x_{1}: y_{1}\right),\left(x_{2}: y_{2}\right),\left(x_{3}: y_{3}\right)\right.$, $\left.\left(x_{4}: y_{4}\right)\right]$ на $\mathbb{P}^{1} \times \mathbb{P}^{1} \times \mathbb{P}^{1} \times \mathbb{P}^{1}$. Тогда $X$ задается уравнением $F\left(x_{1}, y_{1}, x_{2}, y_{2}, x_{3}, y_{3}\right.$, $\left.x_{4}, y_{4}\right)=0$, где $F-$ форма мультистепени $(1,1,1,1)$. Пусть $\pi_{1}: X \rightarrow \mathbb{P}^{1} \times \mathbb{P}^{1} \times \mathbb{P}^{1}$ проекция, заданная как

$\left[\left(x_{1}: y_{1}\right),\left(x_{2}: y_{2}\right),\left(x_{3}: y_{3}\right),\left(x_{4}: y_{4}\right)\right] \mapsto\left[\left(x_{2}: y_{2}\right),\left(x_{3}: y_{3}\right),\left(x_{4}: y_{4}\right)\right] \in \mathbb{P}^{1} \times \mathbb{P}^{1} \times \mathbb{P}^{1}$, а $\pi_{2}, \pi_{3}$ и $\pi_{4}: X \rightarrow \mathbb{P}^{1} \times \mathbb{P}^{1} \times \mathbb{P}^{1}-$ проекции, определяемые аналогичным образом. Положим

$$
F=x_{1} G\left(x_{2}, y_{2}, x_{3}, y_{3}, x_{4}, y_{4}\right)+y_{1} H\left(x_{2}, y_{2}, x_{3}, y_{3}, x_{4}, y_{4}\right),
$$

где $G\left(x_{2}, y_{2}, x_{3}, y_{3}, x_{4}, y_{4}\right)$ и $H\left(x_{2}, y_{2}, x_{3}, y_{3}, x_{4}, y_{4}\right)$ - мультилинейные формы, не зависящие от $x_{1}$ и $y_{1}$. Тогда $\pi_{1}$ является раздутием кривой $C_{1} \subset \mathbb{P}^{1} \times \mathbb{P}^{1} \times \mathbb{P}^{1}$, заданной уравнениями

$$
G\left(x_{2}, y_{2}, x_{3}, y_{3}, x_{4}, y_{4}\right)=H\left(x_{2}, y_{2}, x_{3}, y_{3}, x_{4}, y_{4}\right)=0
$$

которые также определяют поверхность $E_{1} \subset \mathbb{P}^{1} \times \mathbb{P}^{1} \times \mathbb{P}^{1} \times \mathbb{P}^{1}$, стягиваемую 
отображением $\pi_{1}$. Уравнения $x_{1}=H\left(x_{2}, y_{2}, x_{3}, y_{3}, x_{4}, y_{4}\right)=0$ определяют дивизор $H_{1} \subset X$ такой, что $-K_{X} \sim 2 H_{1}+E_{1}$, откуда следует, что $\operatorname{lct}(X) \leqslant 1 / 2$.

Предположим, что $\operatorname{lct}(X)<1 / 2$. Тогда найдется эффективный $\mathbb{Q}$-дивизор $D \sim_{\mathbb{Q}}-K_{X}$ такой, что лог-пара $(X, \lambda D)$ не является лог-канонической при некотором $\lambda<1 / 2$.

Определим дивизоры $E_{2}, E_{3}$ и $E_{4}$ на $X$ аналогично дивизору $E_{1}$. Тогда

$$
\varnothing \neq \operatorname{LCS}(X, \lambda D) \subseteq E_{1} \cap E_{2} \cap E_{3} \cap E_{4},
$$

так как $\operatorname{lct}\left(\mathbb{P}^{1} \times \mathbb{P}^{1} \times \mathbb{P}^{1}\right)=1 / 2$ по лемме 2.21. Но $E_{i} \subset \mathbb{P}^{1} \times \mathbb{P}^{1} \times \mathbb{P}^{1} \times \mathbb{P}^{1}$ задается уравнениями

$$
\frac{\partial F\left(x_{1}, y_{1}, x_{2}, y_{2}, x_{3}, y_{3}, x_{4}, y_{4}\right)}{\partial x_{i}}=\frac{\partial F\left(x_{1}, y_{1}, x_{2}, y_{2}, x_{3}, y_{3}, x_{4}, y_{4}\right)}{\partial y_{i}}=0,
$$

т. е. пересечение $E_{1} \cap E_{2} \cap E_{3} \cap E_{4}$ задается уравнениями

$$
\frac{\partial F}{\partial x_{1}}=\frac{\partial F}{\partial y_{1}}=\frac{\partial F}{\partial x_{2}}=\frac{\partial F}{\partial y_{2}}=\frac{\partial F}{\partial x_{3}}=\frac{\partial F}{\partial y_{3}}=\frac{\partial F}{\partial x_{4}}=\frac{\partial F}{\partial y_{4}}=0 .
$$

Следовательно, $E_{1} \cap E_{2} \cap E_{3} \cap E_{4}=\operatorname{Sing}(X)=\varnothing$ и $\operatorname{LCS}(X, \lambda D)=\varnothing$.

Лемма 9.2. Пусть $I(X)=4.2$. Тогда $\operatorname{lct}(X)=1 / 2$.

ДоказАтеЛьство. Пусть $Q_{1} \subset \mathbb{P}^{4} \supset Q_{2}$ - квадратичные конусы с вершинами $O_{1} \in \mathbb{P}^{4} \ni O_{2}$ соответственно. Выберем гиперплоское сечение $O_{1} \notin S_{1} \subset$ $Q_{1} \subset \mathbb{P}^{4}$ конуса $Q_{1}$. Тогда существует такая неособая эллиптическая кривая $C_{1} \subset\left|-K_{S_{1}}\right|$, что многообразие $X$ включается в коммутативную диаграмму

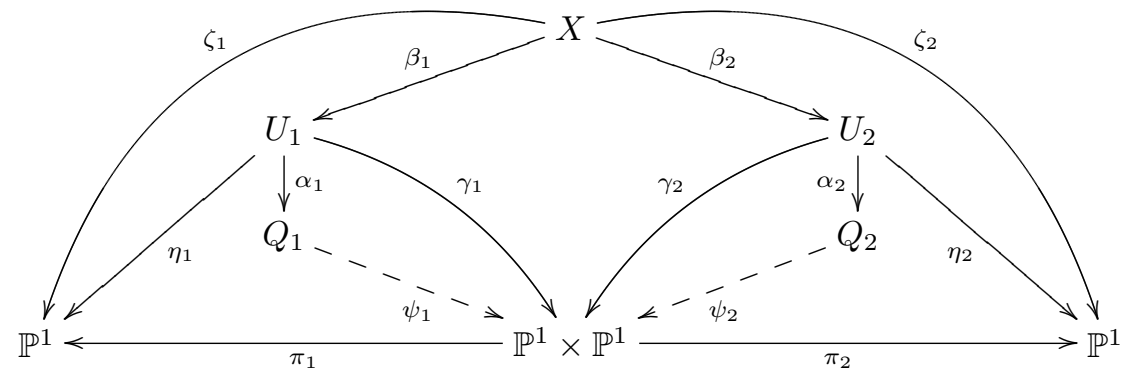

где $\pi_{1} \neq \pi_{2}$ - естественные проекции, отображение $\psi_{i}$ - проекция из точки $O_{i} \in Q_{i} \subset \mathbb{P}^{4}$, морфизм $\alpha_{i}$ - раздутие вершины $O_{i}$ конуса $Q_{i}$, морфизм $\beta_{i}$ стягивает поверхность

$$
\mathbb{P}^{1} \times C_{1} \cong G_{i} \subset X
$$

на кривую $C_{1} \cong C_{i} \subset U_{i}$, морфизм $\eta_{i}$ является $\mathbb{F}_{1}$-расслоением, морфизм $\gamma_{i}$ является $\mathbb{P}^{1}$-расслоением, а морфизм $\zeta_{i}$ - расслоением на поверхности дель Пеццо степени 6 с четырьмя особыми слоями.

Пусть $E_{i} \subset X$ - собственный прообраз исключительного дивизора раздутия $\alpha_{i}$. Тогда поверхности

$$
S_{1}=\alpha_{1} \circ \beta_{1}\left(E_{2}\right) \subset Q_{1} \subset \mathbb{P}^{4} \supset Q_{2} \supset \alpha_{2} \circ \beta_{2}\left(E_{1}\right)
$$

являются гиперплоскими сечениями $Q_{1}$ и $Q_{2}$, содержащими кривые $C_{1}$ и $C_{2}$ соответственно. Также нетрудно видеть, что поверхности $\alpha_{1} \circ \beta_{1}\left(G_{2}\right)$ и $\alpha_{2}$ ○ $\beta_{2}\left(G_{1}\right)$ являются конусами в $\mathbb{P}^{4}$ над кривыми $C_{1}$ и $C_{2}$ соответственно. 
Пусть $\bar{H} \subset X$ - собственный прообраз гиперплоского сечения конуса $Q_{1} \subset \mathbb{P}^{4}$, проходящего через $O_{1}$. Тогда $-K_{X} \sim 2 \bar{H}+E_{2}+E_{1}$, откуда следует неравенство $\operatorname{lct}(X) \leqslant 1 / 2$.

Предположим, что $\operatorname{lct}(X)<1 / 2$. Тогда найдется эффективный $\mathbb{Q}$-дивизор $D \sim_{\mathbb{Q}}-K_{X}$ такой, что лог-пара $(X, \lambda D)$ не лог-канонична при некотором $\lambda<1 / 2$. Положим $D=\mu_{1} E_{1}+\mu_{2} E_{2}+\Omega$, где $\Omega$ - эффективный $\mathbb{Q}$-дивизор на $X$ такой, что $E_{1} \nsubseteq \operatorname{Supp}(\Omega) \nsupseteq E_{2}$.

Пусть $\Gamma$ - общий слой расслоения на коники $\gamma_{1} \circ \beta_{1}$. Тогда

$$
2=\Gamma \cdot D=\Gamma \cdot\left(\mu_{1} E_{1}+\mu_{2} E_{2}+\Omega\right)=\mu_{1}+\mu_{2}+\Gamma \cdot \Omega \geqslant \mu_{1}+\mu_{2},
$$

и без ограничения общности можно считать, что $\mu_{1} \leqslant \mu_{2}$. В этом случае $\mu_{1} \leqslant 1$.

Допустим, что существует поверхность $S \in \mathbb{L} \mathbb{C S}(X, \lambda D)$. Тогда $S \neq E_{1}$. Более того, $S \neq G_{1}$, так как $\alpha_{2} \circ \beta_{2}\left(G_{1}\right)$ является квадратичной поверхностью, и при этом $\lambda<1 / 2$. Следовательно, $S \cap E_{1} \neq \varnothing$. Но $-\left.(1 / 2) K_{E_{1}} \sim_{\mathbb{Q}} D\right|_{E_{1}}$ и $E_{1} \cong \mathbb{P}^{1} \times \mathbb{P}^{1}$, что невозможно по теореме 2.19 и лемме 2.23 . Таким образом, множество $\mathbb{L} \mathbb{C} S(X, \lambda D)$ не содержит поверхностей.

Выберем точку $P \in \operatorname{LCS}(X, \lambda D)$. Предположим, что $P \notin G_{1}$. Пусть $Z$ - слой отображения $\gamma_{1}$ такой, что $\beta_{1}(P) \in Z$. Тогда $Z \subseteq \operatorname{LCS}\left(U_{1}, \lambda \beta_{1}(D)\right)$ по теореме 2.27. Положим $\bar{E}_{1}=\beta_{1}\left(E_{1}\right)$. Тогда $Z \cap \bar{E}_{1} \in \operatorname{LCS}\left(\bar{E}_{1},\left.\lambda \Omega\right|_{\bar{E}_{1}}\right)$ по теореме 2.19, что противоречит лемме 2.23 , так как $\mu_{1} \leqslant 1$.

Таким образом, $P \in G_{1}$. Пусть $F_{1} \subset X \supset F_{2}$ - слои отображений $\zeta_{1}$ и $\zeta_{2}$, проходящие через точку $P$. Тогда либо поверхность $F_{1}$, либо поверхность $F_{2}$ должна быть неособой, так как $\alpha_{1}(P) \in C_{1}$. Но $\operatorname{lct}\left(F_{i}\right)=1 / 2$ в случае, если поверхность $F_{i}$ неособа (см. пример 1.10), что противоречит лемме 2.25 .

Лемма 9.3. Пусть $I(X)=4.3$. Тогда $\operatorname{lct}(X)=1 / 2$.

ДоКАЗАТЕЛЬСТВО. Пусть $F_{1} \cong F_{2} \cong F_{3} \cong \mathbb{P}^{1} \times \mathbb{P}^{1}-$ слои трех различных проекций $\mathbb{P}^{1} \times \mathbb{P}^{1} \times \mathbb{P}^{1} \rightarrow \mathbb{P}^{1}$. Существует стягивание $\alpha: X \rightarrow \mathbb{P}^{1} \times \mathbb{P}^{1} \times \mathbb{P}^{1}$ поверхности $E \subset X$ на кривую $C \subset \mathbb{P}^{1} \times \mathbb{P}^{1} \times \mathbb{P}^{1}$ такую, что $C \cdot F_{1}=C \cdot F_{2}=1$ и $C \cdot F_{3}=2$. Существует неособая поверхность $G \in\left|F_{1}+F_{2}\right|$, содержащая кривую $C$. В частности, выполнено соотношение $-K_{X} \sim 2 \bar{G}+E+\bar{F}_{3}$, где $\bar{F}_{3}$ и $\bar{G}$ - собственные прообразы дивизоров $F_{3}$ и $G$ соответственно. Следовательно, $\operatorname{lct}(X) \leqslant 1 / 2$.

Предположим, что $\operatorname{lct}(X)<1 / 2$. Тогда найдется эффективный $\mathbb{Q}$-дивизор $D \sim_{\mathbb{Q}}-K_{X}$ такой, что лог-пара $(X, \lambda D)$ не лог-канонична при некотором $\lambda<1 / 2$. Заметим, что $\operatorname{LCS}(X, \lambda D) \subseteq E$, так как $\operatorname{lct}\left(\mathbb{P}^{1} \times \mathbb{P}^{1} \times \mathbb{P}^{1}\right)=1 / 2$ и $\alpha(D) \sim_{\mathbb{Q}}-K_{\mathbb{P}^{1} \times \mathbb{P}^{1} \times \mathbb{P}^{1}}$.

Пусть $H \in\left|3 F_{1}+F_{3}\right|-$ неособый дивизор, для которого $C=G \cap H$, а $\bar{H}$ - собственный прообраз дивизора $H$ на многообразии $X$. Тогда $\bar{H} \cap \bar{G}=\varnothing$ и многообразие $X$ включается в коммутативную диаграмму

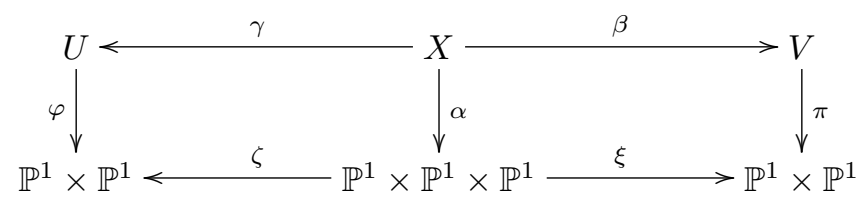


где $\beta$ и $\gamma$ - стягивания поверхностей $\bar{G}$ и $\bar{H}$ на неособые кривые, морфизмы $\pi$ и $\varphi$ являются $\mathbb{P}^{1}$-расслоениями, $\zeta$ и $\xi-$ проекции, заданные линейными системами $\left|F_{1}+F_{2}\right|$ и $\left|F_{1}+F_{3}\right|$ соответственно.

Так как $\bar{H} \cap \bar{G}=\varnothing$, то либо лог-пара $(V, \lambda \beta(D))$ не лог-канонична, либо лог-пара $(U, \lambda \gamma(D))$ не лог-канонична.

Применяя теорему 2.27 к лог-паре $(V, \lambda \beta(D))$ или лог-паре $(U, \lambda \gamma(D))$ (и расслоениям $\pi$ или $\varphi$ соответственно) и используя теорему 2.7 , получаем, что $\operatorname{LCS}(X, \lambda D)=\Gamma$, где $\Gamma-$ слой естественной проекции $E \rightarrow C$.

Можно считать, что $\alpha(\Gamma) \in F_{3}$. Пусть $\bar{F}_{3} \subset X$ - собственный прообраз дивизора $F_{3}$. Положим $D=\mu \bar{F}_{3}+\Omega$, где $\Omega$ - эффективный $\mathbb{Q}$-дивизор на $X$ такой, что $\bar{F}_{3} \not \subset \operatorname{Supp}(\Omega)$. Тогда

$$
\mu F_{3}+\alpha(\Omega) \sim_{\mathbb{Q}} 2\left(F_{1}+F_{2}+F_{3}\right),
$$

откуда следует неравенство $\mu \leqslant 2$. Таким образом, лог-пара $\left(\bar{F}_{3},\left.\lambda \Omega\right|_{\bar{F}_{3}}\right)$ не лог-канонична вдоль кривой $\Gamma \subset \bar{F}_{3}$ по теореме 2.19. Но $\left.\Omega\right|_{\bar{F}_{3}} \sim_{\mathbb{Q}}-K_{\bar{F}_{3}}$ и $\bar{F}_{3}$ является поверхностью дель Пеццо степени $K_{\bar{F}_{3}}^{2}=6$. При этом либо $\bar{F}_{3}$ неособа и $\left|C \cap F_{3}\right|=2$, либо $\bar{F}_{3}$ имеет одну обыкновенную двойную особенность и $\left|C \cap F_{3}\right|=1$.

Заметим, что $\operatorname{lct}\left(\bar{F}_{3}\right) \leqslant \lambda$. Таким образом, поверхность $\bar{F}_{3}$ особа согласно примеру 1.10. Из леммы 4.5 следует, что $\operatorname{LCS}\left(\bar{F}_{3},\left.\lambda \Omega\right|_{\bar{F}_{3}}\right)=\operatorname{Sing}\left(\bar{F}_{3}\right)$, но при этом лог-пара $\left(\bar{F}_{3},\left.\lambda \Omega\right|_{\bar{F}_{3}}\right)$ не лог-канонична во всех точках кривой $\Gamma \subset \bar{F}_{3}$, противоречие.

Лемма 9.4. Пусть $I(X)=4.5$. Тогда $\operatorname{lct}(X)=3 / 7$.

ДокАЗАтельство. Пусть $Q \subset \mathbb{P}^{4}-$ квадратичный конус, $V \subset \mathbb{P}^{6}-$ сечение грассманиана $\operatorname{Gr}(2,5) \subset \mathbb{P}^{9}$ линейным подпространством размерности 6 , имеющее одну обыкновенную двойную особенность. Имеет место коммутативная диаграмма (ср. [61; лемма 2.6])

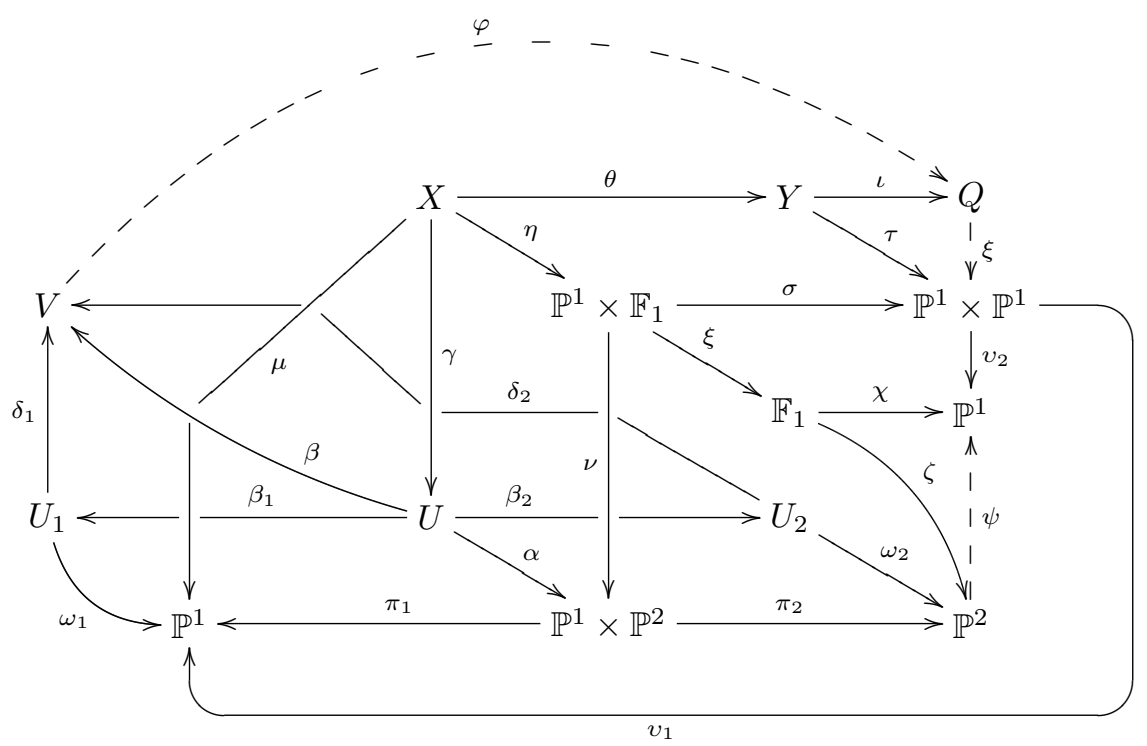


В диаграмме использованы следующие обозначения:

- морфизмы $\pi_{i}, v_{i}, \xi$ и $\chi$ - естественные проекции;

- морфизм $\alpha$ стягивает поверхность $\mathbb{F}_{3} \cong E \subset U$ на кривую $C$, для которой

$$
\pi_{1}^{*}\left(\mathscr{O}_{\mathbb{P}^{1}}(1)\right) \cdot C=2, \quad \pi_{2}^{*}\left(\mathscr{O}_{\mathbb{P}^{2}}(1)\right) \cdot C=1 ;
$$

- морфизм $\beta$ стягивает поверхность $\mathbb{P}^{1} \times \mathbb{P}^{1} \cong \bar{H}_{2} \subset U$ в особую точку многообразия $V$;

- морфизм $\beta_{i}$ стягивает поверхность $\bar{H}_{2}$ на гладкую рациональную кривую;

- морфизм $\delta_{i}$ стягивает кривую $\beta_{i}\left(\bar{H}_{2}\right)$ в особую точку многообразия $V$, так что отображение $\delta_{2} \circ \delta_{1}^{-1}: U_{1} \rightarrow U_{2}$ является стандартным флопом в кривой $\beta_{1}\left(\bar{H}_{2}\right) \cong \mathbb{P}^{1}$;

- морфизм $\omega_{1}$ является расслоением с общим слоем $\mathbb{P}^{1} \times \mathbb{P}^{1}$;

- морфизмы $\omega_{2}, \pi_{2}, \xi, \sigma$ и $\tau$ являются $\mathbb{P}^{1}$-расслоениями;

- морфизм $\zeta$ - раздутие точки $O \in \mathbb{P}^{2}$, не лежащей на кривой $\pi_{2}(C)$;

- отображение $\psi$ - проекция из точки $O \in \mathbb{P}^{2}$;

- морфизм $\nu$ стягивает поверхность $G \cong \mathbb{P}^{1} \times \mathbb{P}^{1}$ на кривую $L$ такую, что $\pi_{2}(L)=O$;

- морфизм $\gamma$ стягивает поверхность $\breve{G}$ на кривую $\bar{L}$ такую, что $\alpha(\bar{L})=$ $L \subset \mathbb{P}^{1} \times \mathbb{P}^{2}$ и кривая $\beta(\bar{L})$ является прямой на $V \subset \mathbb{P}^{6}$, причем $\beta(\bar{L}) \cap$ $\operatorname{Sing}(V)=\varnothing$

- морфизм $\eta$ стягивает на кривую такую поверхность $\breve{E}$, что $\nu \circ \eta(\breve{E})=$ $C \subset \mathbb{P}^{1} \times \mathbb{P}^{2}$

- морфизм $\theta$ стягивает на кривую такую поверхность $\breve{R} \neq \breve{E}$, что $\tau \circ \theta(\breve{R})=$ $\sigma \circ \eta(\breve{E})$

- морфизм $\mu$ - расслоение на поверхности дель Пеццо степени 6;

- морфизм $\iota$ стягивает поверхность $\theta\left(\breve{H}_{2}\right)$ в особую точку квадратичного конуса $Q$;

- отображение $\varphi$ - проекция из прямой $\beta(\bar{L}) \subset V \subset \mathbb{P}^{6}$.

Кривая $\pi_{2}(C) \subset \mathbb{P}^{2}$ является прямой. Поэтому $\alpha\left(\bar{H}_{2}\right) \in\left|\pi_{2}^{*}\left(\mathscr{O}_{\mathbb{P}^{2}}(1)\right)\right|$ и $C \subset$ $\alpha\left(\bar{H}_{2}\right)$.

Морфизм $\pi_{1}$ индуцирует двойное накрытие $C \rightarrow \mathbb{P}^{1}$, разветвленное в двух точках $Q_{1} \in C \ni Q_{2}$. Пусть $T_{i}$ - единственная поверхность в $\left|\pi_{1}^{*}\left(\mathscr{O}_{\mathbb{P}^{1}}(1)\right)\right|$, проходящая через точку $Q_{i}$. Пусть $\bar{T}_{i} \subset U$ - собственный прообраз $T_{i}$. Тогда поверхность $\bar{T}_{i}$

- имеет одну обыкновенную двойную точку,

- касается поверхности $E$ вдоль кривой $E \cap \bar{T}_{i}$,

- является поверхностью дель Пеццо степени $K_{\bar{T}_{i}}^{2}=7$.

Пусть $Z_{i} \subset \mathbb{P}^{2}-$ (единственная) прямая, проходящая через точки $O$ и $\pi_{2} \circ$ $\alpha\left(Q_{i}\right)$. Тогда существует единственная поверхность $\bar{R}_{i} \in\left|\left(\pi_{2} \circ \alpha\right)^{*}\left(\mathscr{O}_{\mathbb{P}^{2}}(1)\right)\right|$ такая, что $Z_{i} \subset \pi_{2} \circ \alpha\left(\bar{R}_{i}\right)$. При этом $\bar{L} \subset \bar{R}_{i}$ и $-K_{U} \sim 2 \bar{H}_{2}+\bar{R}_{i}+2 \bar{T}_{i}+E$.

Пусть $\Gamma_{i}-$ слой проекции $E \rightarrow C$ над точкой $Q_{i}$. Тогда $\Gamma_{i}=E \cap \bar{T}_{i}$ и

$$
\Gamma_{i} \subset \operatorname{LCS}\left(U, \frac{3}{7}\left(2 \bar{H}_{2}+\bar{R}_{i}+2 \bar{T}_{i}+E\right)\right) .
$$


Пусть $\breve{R}_{i}$ и $\breve{T}_{i}$ - собственные прообразы дивизоров $\bar{R}_{i}$ и $\bar{T}_{i}$ на многообразии $X$ соответственно. Тогда $-K_{X} \sim 2 \breve{H}_{2}+\breve{R}_{i}+2 \breve{T}_{i}+\breve{E}$, так как $\bar{L} \subset \bar{R}_{i}$. Пусть $\breve{\Gamma}_{i} \subset X-$ собственный прообраз кривой $\Gamma_{i}$. Тогда лог-пара

$$
\left(X, \frac{3}{7}\left(2 \breve{H}_{2}+\breve{R}_{i}+2 \breve{T}_{i}+\breve{E}\right)\right)
$$

лог-канонична, но не лог-терминальна. Таким образом, $\operatorname{lct}(X) \leqslant 3 / 7$.

Предположим, что $\operatorname{lct}(X)<3 / 7$. Тогда найдется эффективный $\mathbb{Q}$-дивизор $D \sim_{\mathbb{Q}}-K_{X}$ такой, что лог-пара $(X, \lambda D)$ не является лог-канонической при некотором $\lambda<3 / 7$.

Поверхности $\breve{T}_{1}$ и $\breve{T}_{2}$ - единственные особые слои расслоения $\mu: X \rightarrow \mathbb{P}^{1}$. Таким образом,

$$
\breve{T}_{i} \not \subset \operatorname{LCS}(X, \lambda D) \subsetneq \breve{T}_{1} \cup \breve{T}_{2}
$$

по лемме 2.25 , так как $D \cdot Z=\breve{T}_{1}=2$, где $Z$ - общий слой отображения $\pi_{2} \circ \alpha \circ \gamma$.

По теореме 2.7 можно считать, что $\operatorname{LCS}(X, \lambda D) \subseteq \breve{T}_{1}$.

Применяя теорему 2.27 к лог-паре $\left(\mathbb{P}^{1} \times \mathbb{F}_{1}, \lambda \eta(D)\right)$, получаем, что

$$
\operatorname{LCS}(X, \lambda D) \neq \breve{T}_{1} \cap \breve{G}
$$

так как $G=\eta(\breve{G})$ является сечением $\mathbb{P}^{1}$-расслоения $\sigma$.

Применяя теорему 2.27 к лог-паре $\left(\mathbb{P}^{1} \times \mathbb{P}^{2}, \lambda \alpha \circ \gamma(D)\right)$, получаем, что

$$
\varnothing \neq \operatorname{LCS}(X, \lambda D) \subseteq \breve{T}_{1} \cap \breve{E}=\breve{\Gamma}_{1}
$$

по теореме 2.7, так как $\breve{G} \cap \breve{E}=\varnothing$ и $T_{1}$ является сечением расслоения $\pi_{2}$.

Применяя теорему 2.27 к лог-парам $(Y, \lambda \theta(D))$ и $\left(U_{2}, \lambda \beta_{2} \circ \gamma(D)\right)$ (и, соответственно, расслоениям $\tau$ и $\left.\omega_{2}\right)$, получаем, что $\operatorname{LCS}(X, \lambda D)=\breve{\Gamma}_{1}$, так как $\breve{R} \cap \breve{H}_{2}=\varnothing$. Положим $\bar{D}=\gamma(D)$. Тогда $\operatorname{LCS}(U, \lambda \bar{D})=\Gamma_{1}$. Положим $\bar{D}=\varepsilon \bar{H}_{2}+\Omega$, где $\Omega-$ эффективный $\mathbb{Q}$-дивизор такой, что $\bar{H}_{2} \nsubseteq \operatorname{Supp}(\Omega)$. Тогда

$$
\left.\Omega\right|_{\bar{H}_{2}} \sim_{\mathbb{Q}}-\frac{(1+\varepsilon)}{2} K_{\bar{H}_{2}}
$$

и лог-пара $\left(\bar{H}_{2},\left.\lambda \Omega\right|_{\bar{H}_{2}}\right)$ не лог-канонична по теореме 2.19. Отсюда следует, что

$$
\frac{3}{7} \frac{1+\varepsilon}{2}>\lambda \frac{1+\varepsilon}{2}>\frac{1}{2}
$$

по лемме 2.23 , т. е. $\varepsilon>4 / 3$.

Можно считать (см. замечание 2.22 ), что либо $E \nsubseteq \operatorname{Supp}(\bar{D})$, либо $\bar{T}_{1} \nsubseteq$ $\operatorname{Supp}(\bar{D})$.

Допустим, что $E \nsubseteq \operatorname{Supp}(\bar{D})$. Пусть $Z$ - общий слой проекции $E \rightarrow C$. Тогда

$$
1=-K_{U} \cdot Z=\bar{D} \cdot Z=\varepsilon+\Omega \cdot Z \geqslant \varepsilon,
$$

что противоречит неравенству $\varepsilon>4 / 3$. Таким образом, $\bar{T}_{1} \nsubseteq \operatorname{Supp}(\bar{D})$.

Пусть $\bar{\Delta} \subset \bar{T}_{1}-$ собственный прообраз общей прямой на $T_{1} \cong \mathbb{P}^{2}$, проходящей через точку $Q_{1}$. Тогда

$$
2=-K_{U} \cdot \bar{\Delta}=\bar{D} \cdot \bar{\Delta} \geqslant \operatorname{mult}_{\Gamma_{1}}(\bar{D}) \geqslant \frac{1}{\lambda}>\frac{7}{3},
$$


так как $\bar{\Delta} \not \subset \operatorname{Supp}(\bar{D})$ и $\bar{\Delta} \cap \Gamma_{1} \neq \varnothing$. Полученное противоречие завершает доказательство.

Лемма 9.5. Пусть $I(X)=4.6$. Тогда $\operatorname{lct}(X)=1 / 2$.

ДокАЗАТЕЛЬСтво. Напомним, что многообразие $X$ может быть получено как раздутие $\alpha: X \rightarrow \mathbb{P}^{3}$ трех непересекающихся прямых $L_{1}, L_{2}$ и $L_{3}$.

Пусть $H_{i}$ - собственный прообраз на $X$ общей плоскости в $\mathbb{P}^{3}$, содержащей $L_{i}$. Тогда

$$
-K_{X} \sim 2 H_{1}+E_{1}+H_{2}+H_{3} \sim 2 H_{2}+E_{2}+H_{1}+H_{3} \sim 2 H_{3}+E_{3}+H_{1}+H_{2},
$$

где $E_{i}-\alpha$-исключительный дивизор такой, что $\alpha\left(E_{i}\right)=L_{i}$. В частности, $\operatorname{lct}(X) \leqslant 1 / 2$.

Предположим, что $\operatorname{lct}(X)<1 / 2$. Тогда существует эффективный $\mathbb{Q}$-дивизор $D \sim_{\mathbb{Q}}-K_{X}$ такой, что лог-пара $(X, \lambda D)$ не лог-канонична при некотором $\lambda<1 / 2$.

Поверхность $H_{i}$ - неособая поверхностью дель Пеццо, $K_{H_{i}}^{2}=7$, линейная система $\left|H_{i}\right|$ не имеет базисных точек и задает морфизм $\varphi_{i}: X \rightarrow \mathbb{P}^{1}$, все слои которого изоморфны $H_{i}$.

Пусть $|\operatorname{LCS}(X, \lambda D)|<+\infty$. Можно считать, что $\operatorname{LCS}(X, \lambda D) \nsubseteq E_{1}$. Тогда множество

$$
\operatorname{LCS}\left(X, \lambda D+H_{1}+\frac{1}{2} E_{1}\right)
$$

несвязно, что невозможно по теореме 2.7 , так как дивизор $H_{2}+H_{3}+(\lambda-1 / 2) K_{X}$ обилен.

Можно считать, что $H_{1} \cap \operatorname{LCS}(X, \lambda D) \neq \varnothing$. Тогда

$$
\varnothing \neq H_{1} \cap \operatorname{LCS}(X, \lambda D) \subseteq \operatorname{LCS}\left(H_{1},\left.\lambda D\right|_{H_{1}}\right)
$$

в силу замечания 2.3. Положим $C_{2}=\left.E_{2}\right|_{H_{1}}$ и $C_{3}=\left.E_{3}\right|_{H_{1}}$. Тогда $C_{2} \cdot C_{2}=$ $C_{3} \cdot C_{3}=-1$ и существует единственная кривая $\mathbb{P}^{1} \cong C \subset H_{1}$, для которой $C \cdot C_{2}=C \cdot C_{3}=1$ и $C \cdot C=-1$. При этом $\operatorname{LCS}\left(H_{1},\left.\lambda D\right|_{H_{1}}\right)=C$ по лемме 4.9.

Существует единственная неособая квадрика $Q \subset \mathbb{P}^{3}$, содержащая прямые $L_{1}, L_{2}$ и $L_{3}$. Заметим, что $\bar{Q} \cap H_{1}=C$, где $\bar{Q} \subset X-$ собственный прообраз квадрики $Q$.

Существует бирациональный морфизм $\sigma: X \rightarrow \mathbb{P}^{1} \times \mathbb{P}^{1} \times \mathbb{P}^{1}$, стягивающий поверхность $\bar{Q}$ на кривую тристепени $(1,1,1)$. Так как $\bar{Q} \cap H_{1}=C$, то (см. замечание 2.3) $\operatorname{LCS}(X, \lambda D) \supset \bar{Q}$ и, следовательно, $\operatorname{LCS}(X, \lambda D)=\bar{Q}$, так как $\operatorname{lct}\left(\mathbb{P}^{1} \times \mathbb{P}^{1} \times \mathbb{P}^{1}\right)=1 / 2$. Положим $D=\mu \bar{Q}+\Omega$, где $\mu \geqslant 1 / \lambda>2$ и $\Omega-$ эффективный $\mathbb{Q}$-дивизор такой, что $\bar{Q} \not \subset \operatorname{Supp}(\Omega)$. Тогда $\alpha(D)=\mu Q+\alpha(\Omega)$, что невозможно, так как $\alpha(D) \sim_{\mathbb{Q}} 2 Q \sim-K_{\mathbb{P} 3}$ и $\mu>2$.

Лемма 9.6. Пусть $I(X)=4.7$. Тогда $\operatorname{lct}(X)=1 / 2$.

ДокАЗАтельство. Напомним, что многообразие $X$ может быть представлено как раздутие $\alpha: X \rightarrow W$, где $W$ является (неособым) дивизором бистепени $(1,1)$ на $\mathbb{P}^{2} \times \mathbb{P}^{2}$, морфизм $\alpha$ стягивает две (неприводимых) поверхности $E_{1} \neq E_{2}$ на две непересекающиеся кривые $L_{1}$ и $L_{2}$, а кривые $L_{i}$ являются слоями одного и того же естественного $\mathbb{P}^{1}$-расслоения $W \rightarrow \mathbb{P}^{2}$. 
Существует дивизор $H \subset W$ такой, что $-K_{X} \sim 2 H$ и $L_{1} \subset H \supset L_{2}$. Выполнено соотношение $-K_{X} \sim 2 \bar{H}+E_{1}+E_{2}$, где $\bar{H}-$ собственный прообраз $H$ на $X$. Тогда $\operatorname{lct}(X) \leqslant 1 / 2$.

Предположим, что $\operatorname{lct}(X)<1 / 2$. Тогда найдется эффективный $\mathbb{Q}$-дивизор $D \sim_{\mathbb{Q}}-K_{X}$ такой, что особенности лог-пары $(X, \lambda D)$ не являются лог-каноническими при некотором $\lambda<1 / 2$. Заметим, что $\operatorname{LCS}(X, \lambda D) \subseteq E_{1} \cup E_{2}$, так как $\operatorname{lct}(W)=1 / 2$ по теореме 6.1 .

Можно считать, что $\operatorname{LCS}(X, \lambda D) \cap E_{1} \neq \varnothing$. Пусть $\beta: X \rightarrow Y-$ стягивание дивизора $E_{2}$. Тогда $\mathbb{L} \mathbb{C} S(Y, \lambda \beta(D)) \neq \varnothing$ и $\beta(D) \sim_{\mathbb{Q}}-K_{Y}$, что противоречит лемме 8.25 .

Лемма 9.7. Пусть $I(X)=4.8$. Тогда $\operatorname{lct}(X)=1 / 3$.

ДокАЗАТЕЛЬство. Напомним, что $X$ может быть представлено как раздутие $\alpha: X \rightarrow \mathbb{P}^{1} \times \mathbb{P}^{1} \times \mathbb{P}^{1}$ кривой $C \subset \mathbb{P}^{1} \times \mathbb{P}^{1} \times \mathbb{P}^{1}$ такой, что $C \subset F_{1}$ и $C \cdot F_{2}=C \cdot F_{3}=1$, где $F_{i}-$ слой проекции $\mathbb{P}^{1} \times \mathbb{P}^{1} \times \mathbb{P}^{1}$ на $i$-й сомножитель. Существует поверхность $G \in\left|F_{2}+F_{3}\right|$, содержащая кривую $C$. Пусть $E-$ исключительный дивизор раздутия $\alpha$. Тогда $-K_{X} \sim 2 \bar{F}_{1}+2 \bar{G}+3 E$, где $\bar{F}_{1}$ и $\bar{G}$ - собственные прообразы дивизоров $F_{1}$ и $G$ соответственно. В частности, $\operatorname{lct}(X) \leqslant 1 / 3$.

Предположим, что $\operatorname{lct}(X)<1 / 3$. Тогда найдется эффективный $\mathbb{Q}$-дивизор $D \sim_{\mathbb{Q}}-K_{X}$ такой, что лог-пара $(X, \lambda D)$ не лог-канонична при некотором $\lambda<1 / 3$. Заметим, что $\operatorname{LCS}(X, \lambda D) \subseteq E$, так как $\operatorname{lct}\left(\mathbb{P}^{1} \times \mathbb{P}^{1} \times \mathbb{P}^{1}\right)=1 / 2$ и $\alpha(D) \sim_{\mathbb{Q}}-K_{\mathbb{P}^{1} \times \mathbb{P}^{1} \times \mathbb{P}^{1}}$.

Пусть $Q$ - квадратичный конус в $\mathbb{P}^{4}$. Существует коммутативная диаграмма

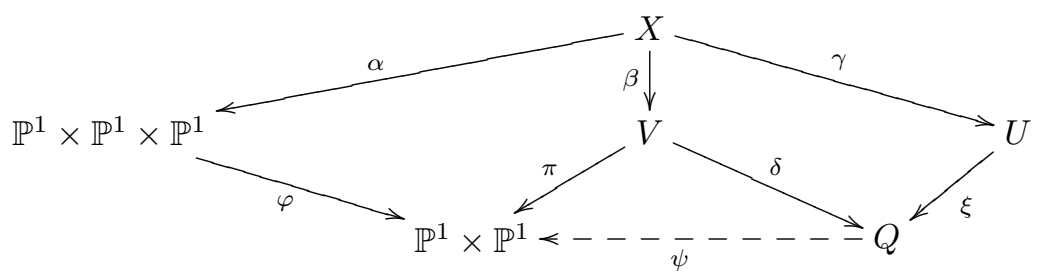

В диаграмме использованы следующие обозначения: $V$ - многообразие с $\beth(V)=$ 3.31 ; морфизм $\beta$ стягивает поверхность $\bar{G}$ на кривую; морфизм $\gamma$ стягивает поверхность $\bar{F}_{1} \cong \mathbb{P}^{1} \times \mathbb{P}^{1}$ в обыкновенную двойную точку; морфизм $\delta$ - раздутие вершины квадратичного конуса $Q \subset \mathbb{P}^{4}$; морфизм $\xi$ - раздутие неособой коники на $Q$; отображение $\psi$ - проекция из вершины конуса $Q$; морфизм $\varphi$ задается линейной системой $\left|F_{2}+F_{3}\right|$, т. е. является проекцией $\mathbb{P}^{1} \times \mathbb{P}^{1} \times \mathbb{P}^{1}$ на произведение двух последних сомножителей; морфизм $\pi$ - естественное $\mathbb{P}^{1}$-расслоение.

Согласно следствию $5.4, \operatorname{lct}(V)=1 / 3$. С другой стороны, $\operatorname{lct}(U)=1 / 3$ по лемме 2.26. Значит, $\operatorname{LCS}(X, \lambda D) \subseteq E \cap \bar{G} \cap \bar{F}_{1}=\varnothing$. Противоречие.

Следующий результат получается из следствий 5.4 и 8.19, леммы 2.29 и примера 1.10.

СлеДСтвиЕ 9.8. Пусть $\rho \geqslant 5$. Тогда $\operatorname{lct}(X)=1 / 3$, если $\beth(X) \in\{5.1,5.2\}$, $u \operatorname{lct}(X)=1 / 2$ в остальных случалх.

Лемма 9.9. Пусть $I(X)=4.13$ и $X$ общее. Тогда $\operatorname{lct}(X)=1 / 2$. 
ДоКАЗАТЕЛЬСТВО. Пусть $F_{1} \cong F_{2} \cong F_{3} \cong \mathbb{P}^{1} \times \mathbb{P}^{1}-$ слои трех различных проекций $\mathbb{P}^{1} \times \mathbb{P}^{1} \times \mathbb{P}^{1} \rightarrow \mathbb{P}^{1}$. Существует стягивание $\alpha: X \rightarrow \mathbb{P}^{1} \times \mathbb{P}^{1} \times \mathbb{P}^{1}$ поверхности $E \subset X$ на кривую $C \subset \mathbb{P}^{1} \times \mathbb{P}^{1} \times \mathbb{P}^{1}$ такую, что $C \cdot F_{1}=C \cdot F_{2}=1$ и $C \cdot F_{3}=3$. Кроме того, существует неособая поверхность $G \in\left|F_{1}+F_{2}\right|$, содержащая $C$. В частности, $-K_{X} \sim 2 \bar{G}+E+2 \bar{F}_{3}$, где $\bar{F}_{3}$ и $\bar{G}-$ собственные прообразы дивизоров $F_{3}$ и $G$ соответственно. Следовательно, выполнено неравенство $\operatorname{lct}(X) \leqslant 1 / 2$.

Предположим, что $\operatorname{lct}(X)<1 / 2$. Тогда найдется эффективный $\mathbb{Q}$-дивизор $D \sim_{\mathbb{Q}}-K_{X}$ такой, что особенности лог-пары $(X, \lambda D)$ не являются лог-каноническими при некотором $\lambda<1 / 2$. Тогда $\operatorname{LCS}(X, \lambda D) \subseteq E \cong \mathbb{F}_{4}$, так как $\operatorname{lct}\left(\mathbb{P}^{1} \times \mathbb{P}^{1} \times \mathbb{P}^{1}\right)=1 / 2$ и $\alpha(D) \sim_{\mathbb{Q}}-K_{\mathbb{P}^{1} \times \mathbb{P}^{1} \times \mathbb{P}^{1}}$.

Отметим, что существуют неособые поверхности $H_{1} \in\left|3 F_{1}+F_{3}\right|$ и $H_{2} \in$ $\left|3 F_{2}+F_{3}\right|$ такие, что $C=G \cdot H_{1}=G \cdot H_{2}$ и $H_{1} \cong H_{2} \cong \mathbb{P}^{1} \times \mathbb{P}^{1}$. Пусть $\bar{H}_{i}-$ собственный прообраз дивизора $H_{i}$ на многообразии $X$. Тогда $\bar{H}_{1} \cap \bar{G}=$ $\bar{H}_{2} \cap \bar{G}=\varnothing$.

Многообразие $X$ включается в коммутативную диаграмму

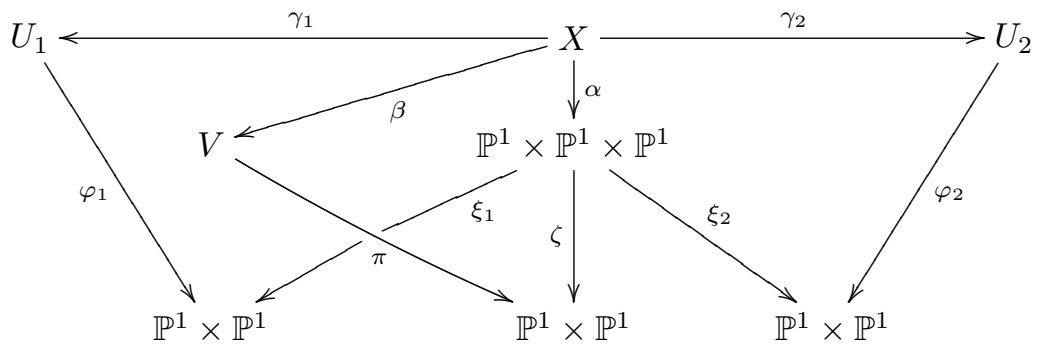

где $\beta$ и $\gamma_{i}$ - стягивания поверхностей $\bar{G}$ и $\bar{H}_{i}$ на неособые кривые соответственно, морфизмы $\pi$ и $\varphi_{i}$ являются $\mathbb{P}^{1}$-расслоениями, морфизмы $\zeta$ и $\xi_{i}-$ проекции, заданные линейными системами $\left|F_{1}+F_{2}\right|$ и $\left|F_{i}+F_{3}\right|$ соответственно.

Так как $\bar{H}_{1} \cap \bar{G}=\varnothing$, то либо лог-пара $(V, \lambda \beta(D))$ не лог-канонична, либо лог-пара $\left(U_{1}, \lambda \gamma_{1}(D)\right)$ не лог-канонична.

Применяя теорему 2.27 к лог-паре $(V, \lambda \beta(D))$ или $\left(U_{1}, \lambda \gamma_{1}(D)\right)$ (и, соответственно, к расслоению $\pi$ или $\left.\varphi_{1}\right)$, с учетом теоремы 2.7 получаем, что $\operatorname{LCS}(X, \lambda D)=\Gamma$, где $\Gamma-$ слой естественной проекции $E \rightarrow C$.

Можно считать, что $\alpha(\Gamma) \in F_{3}$. Положим $D=\mu \bar{F}_{3}+\Omega$, где $\Omega$ - эффективный $\mathbb{Q}$-дивизор на $X$ такой, что $\bar{F}_{3} \not \subset \operatorname{Supp}(\Omega)$. Тогда $\mu F_{3}+\alpha(\Omega) \sim_{\mathbb{Q}} 2\left(F_{1}+F_{2}+F_{3}\right)$, откуда следует неравенство $\mu \leqslant 2$. Лог-пара $\left(\bar{F}_{3},\left.\lambda \Omega\right|_{\bar{F}_{3}}\right)$ не лог-канонична вдоль $\Gamma \subset \bar{F}_{3}$ по теореме 2.19. Заметим, что $\left.\Omega\right|_{\bar{F}_{3}} \sim_{\mathbb{Q}}-K_{\bar{F}_{3}}$ и $\bar{F}_{3}$ является поверхностью дель Пеццо степени $K_{\bar{F}_{3}}^{2}=5$. При этом $\bar{F}_{3}$ может быть особой. А именно,

$$
\operatorname{Sing}\left(\bar{F}_{3}\right)=\varnothing \Longleftrightarrow\left|C \cap F_{3}\right|=F_{3} \cdot C=3,
$$

и $\operatorname{Sing}\left(\bar{F}_{3}\right) \subset \Gamma$. Возможны следующие случаи:

- поверхность $\bar{F}_{3}$ неособа и $\left|C \cap F_{3}\right|=3$;

- поверхность $\bar{F}_{3}$ имеет одну обыкновенную двойную особенность и выполнено равенство $\left|C \cap F_{3}\right|=2$;

- поверхность $\bar{F}_{3}$ имеет особую точку типа $\mathbb{A}_{2}$ и $\left|C \cap F_{3}\right|=1$. 
Заметим, что $\operatorname{lct}\left(\bar{F}_{3}\right) \leqslant \lambda<1 / 2$. Таким образом, из примеров 1.10 и 4.3 следует, что $\left|C \cap F_{3}\right|=1$; последняя возможность не реализуется на общем $X$.

\section{0. Оценки сверху}

Мы будем пользоваться предположениями и обозначениями, введенными в разделе 1. Основная цель данного раздела - оценить сверху глобальные лог-канонические пороги многообразия $X$ в некоторых случаях, не покрытых теоремой 1.46 .

ЛЕмма 10.1. Пусть $I(X)=1.8$. Тогда $\operatorname{lct}(X) \leqslant 6 / 7$.

ДокАЗАТЕЛЬство. Линейная система $\left|-K_{X}\right|$ не имеет базисных точек и задает вложение $X \subset \mathbb{P}^{10}$, причем многообразие $X$ содержит прямую $L \subset X$ (см. [62]).

Из [2; теорема 4.3.3] следует, что $X$ включается в коммутативную диаграмму

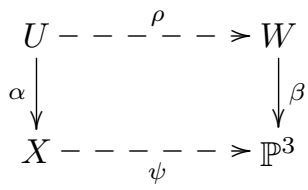

где $\alpha$ - раздутие прямой $L$, отображение $\rho$ - композиция флопов, $\beta$ - раздутие гладкой кривой степени 7 рода 3 , а $\psi$ - двойная проекция из прямой $L$.

Пусть $S \subset X$ - собственный прообраз исключительного дивизора раздутия $\beta$. Тогда выполнено неравенство $\operatorname{mult}_{L}(S)=7$ и $S \sim-3 K_{X}$, откуда следует $\operatorname{lct}(X) \leqslant 6 / 7$.

Лемма 10.2. Пусть $I(X)=1.9$. Тогда $\operatorname{lct}(X) \leqslant 4 / 5$.

ДокАзАтельство. Линейная система $\left|-K_{X}\right|$ не имеет базисных точек и задает вложение $X \subset \mathbb{P}^{11}$, причем многообразие $X$ содержит прямую $L \subset X$ (см. [62]).

Из [2; теорема 4.3.3] следует, что $X$ включается в коммутативную диаграмму

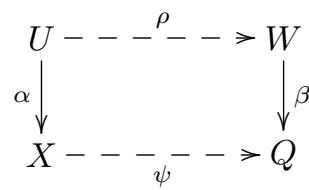

где $Q \subset \mathbb{P}^{4}$ - гладкая квадрика, $\alpha$ - раздутие прямой $L$, отображение $\rho-$ композиция флопов, $\beta$ - раздутие неособой кривой степени 7 рода 2 , а $\psi-$ двойная проекция из $L$.

Пусть $S \subset X$ - собственный прообраз исключительного дивизора раздутия $\beta$. Тогда выполнено неравенство $\operatorname{mult}_{L}(S)=5$ и $S \sim-2 K_{X}$, откуда следует $\operatorname{lct}(X) \leqslant 4 / 5$.

Лемма 10.3. Пусть $I(X)=1.10$. Тогда $\operatorname{lct}(X) \leqslant 2 / 3$.

ДокАзАтЕЛьство. Линейная система $\left|-K_{X}\right|$ не имеет базисных точек и задает вложение $X \subset \mathbb{P}^{13}$, причем многообразие $X$ содержит прямую $L \subset X$ (см. [62]). 
Из [2; теорема 4.3.3] следует, что $X$ включается в коммутативную диаграмму

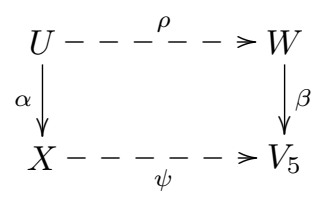

где $V_{5}$ - неособое сечение грассманиана $\operatorname{Gr}(2,5) \subset \mathbb{P}^{9}$ линейным подпространством размерности 6 , морфизм $\alpha$ - раздутие прямой $L$, отображение $\rho$ является композицией флопов, $\beta$ - раздутие рациональной нормальной кривой степени 5 , а $\psi$ - двойная проекция из прямой $L$.

Пусть $S \subset X$ - собственный прообраз исключительного дивизора раздутия $\beta$. Тогда $\operatorname{mult}_{L}(S)=3$ и $S \sim-K_{X}$, откуда $\operatorname{lct}(X) \leqslant 2 / 3$.

Лемма 10.4. Пусть $\beth(X)=2.2$. Тогда $\operatorname{lct}(X) \leqslant 13 / 14$.

ДокАЗАтельство. Существует неособый дивизор $B \subset \mathbb{P}^{1} \times \mathbb{P}^{2}$ бистепени $(2,4)$ такой, что многообразие $X$ включается в коммутативную диаграмму

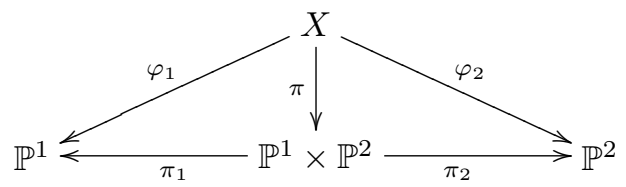

где $\pi$ - двойное накрытие с ветвлением в $B$, морфизмы $\pi_{1}$ и $\pi_{2}$ - естественные проекции, $\varphi_{1}$ - расслоение на поверхности дель Пеццо степени 2 , а $\varphi_{2}-$ расслоение на коники.

Пусть $H_{1}$ - общий слой расслоения $\varphi_{1}$. Положим $\bar{H}_{1}=\pi\left(H_{1}\right)$. Тогда пересечение

$$
C=\bar{H}_{1} \cap B \subset \bar{H}_{1} \cong \mathbb{P}^{2}
$$

является неособой кривой степени 4.

Существует точка $P \in C$, для которой $\operatorname{mult}_{P}(C \cdot L) \geqslant 3$, где $L \subset \bar{H}_{1} \cong \mathbb{P}^{2}-$ касательная прямая к кривой $C$ в точке $P$.

Кривая $\pi_{2}(L)$ является прямой. Таким образом, существует единственная поверхность $H_{2} \in\left|\varphi_{2}^{*}\left(\mathscr{O}_{\mathbb{P}^{2}}(1)\right)\right|$ такая, что $\varphi_{2}\left(H_{2}\right)=\pi_{2}(L)$. Следовательно, $-K_{X} \sim H_{1}+H_{2}$.

Покажем, что $\operatorname{lct}\left(X, H_{1}+H_{2}\right) \leqslant 13 / 14$. Положим $\bar{H}_{2}=\pi\left(H_{2}\right)$. Тогда

$$
\mathbb{L} \mathbb{C S}\left(X, \frac{13}{14}\left(H_{1}+H_{2}\right)\right) \neq \varnothing \Longleftrightarrow \mathbb{L} \mathbb{C S}\left(\mathbb{P}^{1} \times \mathbb{P}^{2}, \frac{1}{2} B+\frac{13}{14}\left(\bar{H}_{1}+\bar{H}_{2}\right)\right) \neq \varnothing
$$

по [1; предложение 3.16]. Пусть $\alpha: V \rightarrow \mathbb{P}^{1} \times \mathbb{P}^{2}-$ раздутие кривой $C$. Тогда

$$
K_{V}+\frac{1}{2} \widetilde{B}+\frac{13}{14}\left(\widetilde{H}_{1}+\widetilde{H}_{2}\right)+\frac{3}{7} E \sim_{\mathbb{Q}} \alpha^{*}\left(K_{\mathbb{P}^{1} \times \mathbb{P}^{2}}+\frac{1}{2} B+\frac{13}{14}\left(\bar{H}_{1}+\bar{H}_{2}\right)\right),
$$

где $\widetilde{B}, \widetilde{H}_{1}, \widetilde{H}_{2} \subset V$ - собственные прообразы $B, \bar{H}_{1}, \bar{H}_{2}$ соответственно. При этом особенности лог-пары $\left(V,(13 / 14) \widetilde{H}_{2}+(3 / 7) E\right)$ не являются лог-терминальными вдоль слоя $Г$ морфизма $\alpha$ такого, что $\alpha(\Gamma)=P$, так как

$$
\operatorname{mult}_{\Gamma}\left(\widetilde{H}_{2} \cdot E\right)=\operatorname{mult}_{P}\left(C \cdot \bar{H}_{2}\right) \geqslant \operatorname{mult}_{P}(C \cdot L) \geqslant 3
$$


из-за общности в выборе слоя $H_{1}$. Таким образом,

$$
\Gamma \subseteq \operatorname{LCS}\left(V, \frac{13}{14} \widetilde{H}_{2}+\frac{3}{7} E\right) \subseteq \operatorname{LCS}\left(V, \frac{1}{2} \widetilde{B}+\frac{13}{14}\left(\widetilde{H}_{1}+\widetilde{H}_{2}\right)+\frac{3}{7} E\right),
$$

откуда получаем неравенство $\operatorname{lct}\left(X, H_{1}+H_{2}\right) \leqslant 13 / 14$. Следовательно, выполняется и неравенство $\operatorname{lct}(X) \leqslant 13 / 14$.

ЗАмечАнИЕ 10.5. Из лемм 2.25 и 4.1 следует, что $\operatorname{lct}(X) \geqslant 2 / 3$, если $I(X)=2.2$ и многообразие $X$ настолько общее, что для любого слоя расслоения $\varphi_{1}$ выполнены условия леммы 4.1 .

Лемма 10.6. Пусть $I(X)=2.7$. Тогда $\operatorname{lct}(X) \leqslant 2 / 3$.

ДокАзАтЕльство. Многообразие $X$ включается в коммутативную диаграмму

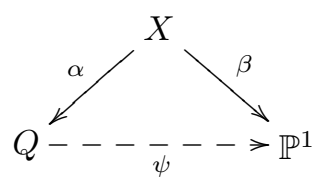

где $Q \subset \mathbb{P}^{4}$ - неособая квадрика, $\alpha$ - раздутие неособой кривой, являющейся полным пересечением двух дивизоров $S_{1}, S_{2} \in\left|\mathscr{O}_{\mathbb{P}^{4}}(2)\right|_{Q} \mid$, морфизм $\beta$ является расслоением на поверхности дель Пеццо степени 4 , а $\psi$ - рациональное отображение, заданное пучком, порожденным дивизорами $S_{1}$ и $S_{2}$. Отсюда следует, что $\operatorname{lct}(X) \leqslant 2 / 3$, так как $-K_{X} \sim_{\mathbb{Q}}(3 / 2) \bar{S}_{1}+(1 / 2) E$, где $\bar{S}_{1} \subset X-$ собственный прообраз поверхности $S_{1}$, а $E$ - исключительный дивизор раздутия $\alpha$.

Лемма 10.7. Пусть $I(X)=2.9$. Тогда $\operatorname{lct}(X) \leqslant 3 / 4$.

ДокАзАтельство. Многообразие $X$ включается в коммутативную диаграмму

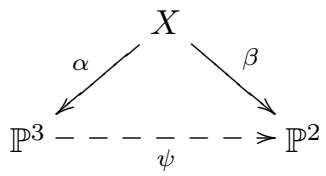

где $\alpha$ - раздутие неособой кривой $C \subset \mathbb{P}^{3}$ степени 7 рода 5 , являющейся пересечением кубик в $\mathbb{P}^{3}$, морфизм $\beta$ - расслоение на коники, а $\psi$ - рациональное отображение, заданное линейной системой кубик, проходящих через кривую $C$. При этом выполнено соотношение $-K_{X} \sim_{\mathbb{Q}}(4 / 3) S+(1 / 3) E$, где $S \in\left|\beta^{*}\left(\mathscr{O}_{\mathbb{P}^{2}}(1)\right)\right|$, а $E-$ исключительный дивизор раздутия $\alpha$. Следовательно, $\operatorname{lct}(X) \leqslant 3 / 4$.

Лемма 10.8. Пусть $I(X)=2.12$. Тогда $\operatorname{lct}(X) \leqslant 3 / 4$.

ДокАзАТЕльство. Многообразие $X$ включается в коммутативную диаграмму

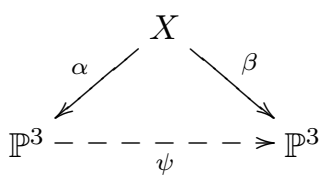


где $\alpha$ и $\beta$ - раздутия неособых кривых $C \subset \mathbb{P}^{3}$ и $Z \subset \mathbb{P}^{3}$ степени 6 рода 3 , являющихся пересечениями кубик в $\mathbb{P}^{3}$, а $\psi$ - бирациональное отображение, заданное линейной системой кубик, проходящих через кривую $C$. При этом $-K_{X} \sim_{\mathbb{Q}}(4 / 3) S+(1 / 3) E$, где $S \in\left|\beta^{*}\left(\mathscr{O}_{\mathbb{P} 3}(1)\right)\right|$, а $E$ - исключительный дивизор раздутия $\alpha$. Следовательно, $\operatorname{lct}(X) \leqslant 3 / 4$.

Лемма 10.9. Пусть $I(X)=2.13$. Тогда $\operatorname{lct}(X) \leqslant 2 / 3$.

ДокАзАтельство. Многообразие $X$ включается в коммутативную диаграмму

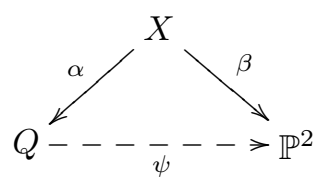

где $Q \subset \mathbb{P}^{4}$ - неособая квадрика, $\alpha$ - раздутие неособой кривой $C \subset Q$ степени 6 рода 2 , морфизм $\beta$ - расслоение на коники, а $\psi$ - рациональное отображение, заданное линейной системой поверхностей из $\left|\mathscr{O}_{\mathbb{P}^{4}}(2)\right|_{Q} \mid$, проходящих через кривую $C$. При этом выполнено соотношение $-K_{X} \sim_{\mathbb{Q}}(3 / 2) S+(1 / 2) E$, где $S \in\left|\beta^{*}\left(\mathscr{O}_{\mathbb{P}^{2}}(1)\right)\right|$, а $E$ - исключительный дивизор раздутия $\alpha$. Следовательно, $\operatorname{lct}(X) \leqslant 2 / 3$.

Лемма 10.10. Пусть $I(X)=2.16$. Тогда $\operatorname{lct}(X) \leqslant 1 / 2$.

ДокАзАтельство. Многообразие $X$ включается в коммутативную диаграмму

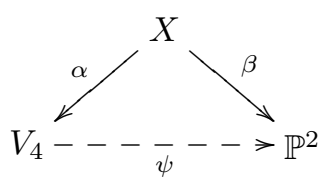

где $V_{4} \subset \mathbb{P}^{5}$ - неособое полное пересечение двух квадрик, $\alpha$ - раздутие неособой коники $C \subset V_{4}$, морфизм $\beta$ - расслоение на коники, а $\psi$ - рациональное отображение, заданное линейной системой поверхностей из $\left|\mathscr{O}_{\mathbb{P}^{5}}(1)\right|_{V_{4}} \mid$, проходящих через кривую $C$. При этом выполнено соотношение $-K_{X} \sim 2 S+E$, где $S \in\left|\beta^{*}\left(\mathscr{O}_{\mathbb{P}^{2}}(1)\right)\right|$, а $E-$ исключительный дивизор раздутия $\alpha$. Следовательно, $\operatorname{lct}(X) \leqslant 1 / 2$.

Лемма 10.11. Пусть $\beth(X)=2.17$. Тогда $\operatorname{lct}(X) \leqslant 2 / 3$.

ДокАЗАтельство. Многообразие $X$ включается в коммутативную диаграмму

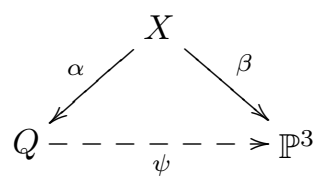

где $Q \subset \mathbb{P}^{4}$ - неособая квадрика, $\alpha$ и $\beta$ - раздутия неособых эллиптических кривых $C \subset Q$ и $Z \subset \mathbb{P}^{3}$ степени 5 соответственно, а отображение $\psi$ задается линейной системой поверхностей в $\left|\mathscr{O}_{\mathbb{P}^{4}}(2)\right|_{Q} \mid$, проходящих через кривую $C$. При этом выполнено соотношение $-K_{X} \sim_{\mathbb{Q}}(3 / 2) S+(1 / 2) E$, где $S \in\left|\beta^{*}\left(\mathscr{O}_{\mathbb{P}^{3}}(1)\right)\right|$, а $E$ - исключительный дивизор раздутия $\alpha$. Следовательно, $\operatorname{lct}(X) \leqslant 2 / 3$. 
Лемма 10.12. Пусть $I(X)=2.20$. Тогда $\operatorname{lct}(X) \leqslant 1 / 2$.

ДокАзАтельство. Многообразие $X$ включается в коммутативную диаграмму

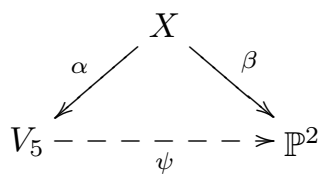

где $V_{5} \subset \mathbb{P}^{6}$ - неособое сечение грассманиана $\operatorname{Gr}(2,5) \subset \mathbb{P}^{9}$ линейным подпространством размерности 6 , морфизм $\alpha$ - раздутие скрученной кубики $\mathbb{P}^{1} \cong$ $C \subset V_{5}$, морфизм $\beta$ - расслоение на коники, а отображение $\psi$ задается линейной системой поверхностей из $\left|\mathscr{O}_{\mathbb{P}^{6}}(1)\right|_{V_{5}} \mid$, проходящих через кривую $C$. При этом $-K_{X} \sim 2 S+E$, где $S \in\left|\beta^{*}\left(\mathscr{O}_{\mathbb{P}^{2}}(1)\right)\right|$, а $E$ - исключительный дивизор раздутия $\alpha$. Следовательно, $\operatorname{lct}(X) \leqslant 1 / 2$.

Лемма 10.13. Пусть $I(X)=2.21$. Тогда $\operatorname{lct}(X) \leqslant 2 / 3$.

ДокАзАтельство. Многообразие $X$ включается в коммутативную диаграмму

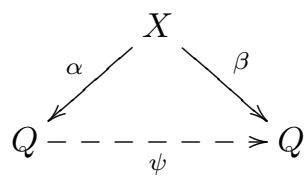

где $Q \subset \mathbb{P}^{4}$ - неособая квадрика, $\alpha$ и $\beta$ - раздутия нормальных рациональных кривых $C \subset Q$ и $Z \subset Q$ степени 4, а $\psi$ - бирациональное отображение, заданное линейной системой поверхностей из $\left|\mathscr{O}_{\mathbb{P}^{4}}(2)\right|_{Q} \mid$, проходящих через кривую $C$. При этом выполнено соотношение $-K_{X} \sim_{\mathbb{Q}}(3 / 2) S+(1 / 2) E$, где $S \in\left|\beta^{*}\left(\mathscr{O}_{\mathbb{P}^{4}}(1)\right)\right|_{Q} \mid$, а $E$ - исключительный дивизор раздутия $\alpha$. Следовательно, $\operatorname{lct}(X) \leqslant 2 / 3$.

Лемма 10.14. Пусть $\beth(X)=2.22$. Тогда $\operatorname{lct}(X) \leqslant 1 / 2$.

ДокАзАтельство. Многообразие $X$ включается в коммутативную диаграмму

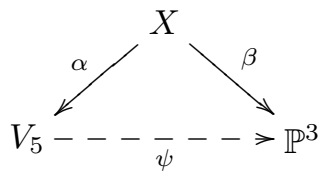

где $V_{5} \subset \mathbb{P}^{6}$ - неособое сечение грассманиана $\operatorname{Gr}(2,5) \subset \mathbb{P}^{9}$ линейным подпространством размерности 6 , морфизмы $\alpha$ и $\beta$ - раздутия коники $C \subset V_{5}$ и (не линейно нормальной) рациональной квартики $Z \subset \mathbb{P}^{3}$ соответственно, а отображение $\psi$ задается линейной системой поверхностей из $\left|\mathscr{O}_{\mathbb{P} 6}(1)\right|_{V_{5}} \mid$, проходящих через конику $C$. При этом выполнено соотношение $-K_{X} \sim 2 S+E$, где $S \in\left|\beta^{*}\left(\mathscr{O}_{\mathbb{P}^{3}}(1)\right)\right|$, а $E-$ исключительный дивизор раздутия $\alpha$. Следовательно, $\operatorname{lct}(X) \leqslant 1 / 2$.

Лемма 10.15. Пусть $I(X)=3.13$. Тогда $\operatorname{lct}(X) \leqslant 1 / 2$. 
ДокАЗАтельство. Многообразие $X$ включается в коммутативную диаграмму

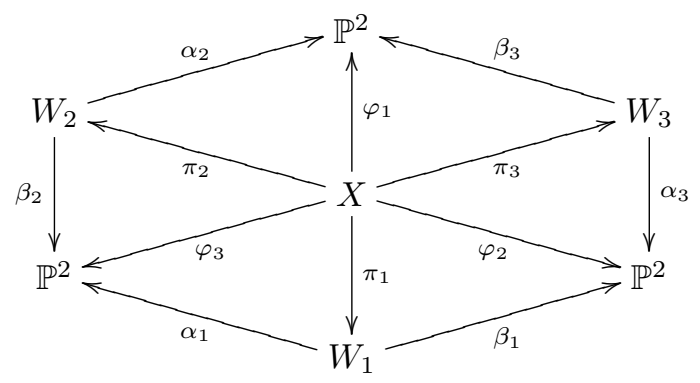

где $W_{i} \subset \mathbb{P}^{2} \times \mathbb{P}^{2}$ - дивизор бистепени $(1,1)$, морфизмы $\alpha_{i}$ и $\beta_{i}$ являются $\mathbb{P}^{1}$-расслоениями, $\pi_{i}$ - раздутие неособой кривой $C_{i} \subset W_{i}$ бистепени $(2,2)$ такой, что образы $\alpha_{i}\left(C_{i}\right)$ и $\beta_{i}\left(C_{i}\right)$ являются (неприводимыми) кониками на $\mathbb{P}^{2}$, а $\varphi_{i}$ - расслоение на коники. Пусть $E_{i}$ - исключительный дивизор раздутия $\pi_{i}$. Тогда

$$
-K_{X} \sim 2 H_{1}+E_{1} \sim 2 H_{2}+E_{2} \sim 2 H_{3}+E_{3} \sim E_{1}+E_{2}+E_{3},
$$

где $H_{i} \in\left|\varphi_{i}^{*}\left(\mathscr{O}_{\mathbb{P}^{2}}(1)\right)\right|$. Следовательно, $\operatorname{lct}(X) \leqslant 1 / 2$.

ЗАмечАнИЕ 10.16. Примем обозначения, введенные в доказательстве леммы 10.15 , и допустим, что $\operatorname{lct}(X)<1 / 2$. Тогда найдется эффективный $\mathbb{Q}$-дивизор $D \sim_{\mathbb{Q}}-K_{X}$ такой, что лог-пара $(X, \lambda D)$ не лог-канонична при некотором $\lambda<1 / 2$. Так как $\operatorname{lct}\left(W_{i}\right)=1 / 2$ по теореме 6.1 , то

$$
\varnothing \neq \operatorname{LCS}(X, \lambda D) \subset E_{1} \cap E_{2} \cap E_{3} .
$$

В частности, по теореме 2.7 множество $\operatorname{LCS}(X, \lambda D)$ состоит из единственной точки $P$; заметим, что точка $P$ является пересечением $P=F_{1} \cap F_{2} \cap F_{3}$ трех кривых $F_{i}$ таких, что $F_{2} \cup F_{3}$ (соответственно $F_{1} \cup F_{3}, F_{1} \cup F_{2}$ ) является приводимым слоем расслоения на коники $\varphi_{1}$ (соответственно $\left.\varphi_{2}, \varphi_{3}\right)$.

\section{Приложение А.}

\section{Ж.-П. Демайи. Инвариант Тиана и лог-канонические пороги}

Цель настоящего приложения - показать связь между глобальными лог-каноническими порогами и $\alpha$-инвариантами неособых многообразий Фано, которые были введены в работе [3] для изучения вопроса существования метрик Кэлера-Эйнштейна на неособых многообразиях Фано. Мы воспользуемся техникой приближения замкнутых положительных $(1,1)$-потоков, которая была разработана в [63], и покажем, что $\alpha$-инвариант неособого многообразия Фано совпадает с его глобальным лог-каноническим порогом.

После появления работы [21] многим алгебраическим геометрам было понятно, что $\alpha$-инвариант неособого многообразия Фано совпадает с его глобальным лог-каноническим порогом. Более того, несколько работ неявно использовали это утверждение (см., например, [64] и [65]). Однако требуемый результат был явно сформулирован только в локально-аналитической форме в работе [21], 
причем с использованием терминологии, незнакомой большинству алгебраических геометров. В настоящем приложении мы исправим этот пробел, сформулировав и доказав утверждение о существовании метрик Кэлера-Эйнштейна на многообразиях Фано.

Практически во всех применениях рассматриваются объекты, связанные с антиканоническим линейным расслоением $L=-K_{X}$. Тем не менее, мы рассмотрим более общий случай произвольного линейного расслоения $L$ (или $\mathbb{Q}$-линейного расслоения $L$ ) на неособом комплексном многообразии $X$, которое удовлетворяет некоторым естественным дополнительным ограничениям, сформулированным ниже.

Предположим, что линейное расслоение $L$ оснащено особой эрмитовой метpикой $h$ (см. [66]). Линейное расслоение $L$ локально допускает тривиализацию $\theta:\left.L\right|_{U} \simeq U \times \mathbb{C}$ такую, что на открытом подмножестве $U$ метрика $h$ задается весовой функцией $\varphi$, для которой

$$
\|\xi\|_{h}^{2}=|\xi|^{2} e^{-2 \varphi(z)} \quad \text { при всех } z \in U, \xi \in L_{z},
$$

где мы отождествляем $\xi \in L_{z}$ с комплексным числом. Нам интересен случай, когда функция $\varphi$ является (по крайней мере) локально интегрируемой по Лебегу функцией, поскольку в этом случае мы можем посчитать форму кривизны

$$
\Theta_{L, h}=\frac{i}{\pi} \partial \bar{\partial} \varphi
$$

в смысле распределения. В этом случае $\Theta_{L, h} \geqslant 0$ как $(1,1)$-поток, если и только если функция $\varphi$ является плюрисубгармонической. В дальнейшем нам будет интересен только этот случай.

Рассмотрим понятие комплексного показателя особенности для особых эрмитовых метрик, следуя работам [67]-[69] и [21].

ОПРеДЕЛЕНиЕ А.1. Для компактного подмножества $K$ многообразия $X$ комплексным показателем особенности $c_{K}(h)$ метрики $h$, которая может быть локально представлена в виде $h=e^{-2 \varphi}$, принято называть супремум всех положительных чисел $c \in \mathbb{R}$ таких, что в окрестности каждой точки $z_{0} \in K$ функция $h^{c}=e^{-2 c \varphi}$ является интегрируемой по мере Лебега в голоморфных координатах с началом в точке $z_{0}$.

Теперь мы рассмотрим обобщение $\alpha$-инварианта Тиана, который был введен в работе [3] (см. также [70]).

ОПРеДЕЛЕНиЕ А.2. Предположим, что многообразие $X$ компактно, а линейное расслоение $L$ псевдоэффективно, т. е. $L$ обладает особой эрмитовой метрикой $h_{0}$ такой, что $\Theta_{L, h_{0}} \geqslant 0$. Для компактного подмножества $K \subset X$ положим

$$
\alpha_{K}(L)=\inf _{\left\{h, \Theta_{L, h} \geqslant 0\right\}} c_{K}(h),
$$

где инфимум берется по всем особым эрмитовым метрикам $h$ на линейном расслоении $L$ таким, что $\Theta_{L, h} \geqslant 0$.

В алгебраической геометрии важным объектом изучения является линейная система, определяемая некоторым набором линейно независимых сечений 
$\sigma_{0}, \sigma_{1}, \ldots, \sigma_{N} \in H^{0}\left(X, L^{\otimes m}\right)$. Обозначим символом $\Sigma$ векторное пространство, порожденное такими сечениями. Положим

$$
|\Sigma|:=P(\Sigma) \subset|m L|:=P\left(H^{0}\left(X, L^{\otimes m}\right)\right)
$$

и отметим, что линейная система $|\Sigma|$ в общем случае не обязательно является полной. Такой набор из $N+1$ сечения $\sigma=\left(\sigma_{j}\right)_{0 \leqslant j \leqslant N}$ естественным образом задает особую эрмитову метрику $h$ на линейном расслоении $L$. А именно, для любой тривиализации положим

$$
\|\xi\|_{h}^{2}=\frac{|\xi|^{2}}{\left(\sum_{j}\left|\sigma_{j}(z)\right|^{2}\right)^{1 / m}}=\frac{|\xi|^{2}}{|\sigma(z)|^{2 / m}} \quad \text { при } \xi \in L_{z}
$$

откуда следует, что $h(z)=|\sigma(z)|^{-2 / m}$ с ассоциированной весовой функцией

$$
\varphi(z)=\frac{1}{m} \log |\sigma(z)|=\frac{1}{2 m} \log \sum_{j}\left|\sigma_{j}(z)\right|^{2} .
$$

Нас интересует значение числа $c_{K}\left(|\sigma|^{-2 / m}\right)$. В случае единственного сечения $\sigma_{0}$ (соответствующего линейной системе, состоящей из одного дивизора) это число совпадает с лог-каноническим порогом $\operatorname{lct}_{K}\left(X, m^{-1} D\right)$, где $D$ - дивизор нулей сечения $\sigma_{0}$. Для линейной системы $|\Sigma| \subset|m L|$ произвольной размерности мы будем обозначать число $c_{K}\left(|\sigma|^{-2 / m}\right)$ символом $\operatorname{lct}_{K}\left(X, m^{-1}|\Sigma|\right)$.

Напомним, что линейное расслоение $L$ принято называть объемным, если его размерность Кодаиры-Иитаки $\kappa(L)$ равна размерности многообразия $n=$ $\operatorname{dim}_{\mathbb{C}}(X)$. Основным результатом настоящего приложения является следующая теорема.

Теорема А.3. Пусть $L$ - объемное линейное расслоение на компактном комплексном многообразии $X$. Тогда для каждого компактного подмножества $K \subset X$ выполнено равенство

$$
\alpha_{K}(L)=\inf _{\left\{h, \Theta_{L, h} \geqslant 0\right\}} c_{K}(h)=\inf _{m \in \mathbb{Z}_{>0}} \inf _{D \in|m L|} \operatorname{lct}_{K}\left(X, \frac{1}{m} D\right) .
$$

Отметим, что очевидным образом выполнено неравенство

$$
\inf _{m \in \mathbb{Z}_{>0}} \inf _{D \in|m L|} \operatorname{lct}_{K}\left(X, \frac{1}{m} D\right) \geqslant \inf _{\left\{h, \Theta_{L, h} \geqslant 0\right\}} c_{K}(h),
$$

поскольку каждый дивизор $D \in|m L|$ естественным образом задает особую эрмитову метрику $h$. Мы покажем, что выполнено и обратное неравенство, используя технику приближения работы [63] и некоторый элементарный анализ. Отметим, что наше доказательство полностью аналогично доказательству теоремы 4.2 в [21], хотя используемая в этих двух случаях терминология немного различается. Однако в обоих случаях используется одна и та же идея из теории пучков множительных идеалов: для особой эрмитовой метрики $h$ 
с локальной плюрисубгармонической весовой функцией $\varphi$ пучок множительных идеалов $\mathscr{I}(h) \subset \mathscr{O}_{X}$ (также обозначаемый символом $\mathscr{I}(\varphi)$ ) является пучком идеалов, который может быть определен как

$$
\begin{aligned}
& \mathscr{I}(h)_{z}=\left\{f \in \mathscr{O}_{X, z} \mid\right. \text { найдется окрестность } V \ni z \\
&\text { такая, что } \left.\int_{V}|f(x)|^{2} e^{-2 \varphi(x)} d \lambda(x)<+\infty\right\},
\end{aligned}
$$

где $\lambda$ - мера Лебега. Как показано А. М. Наделем (см. [20]), этот пучок является когерентным аналитическим пучком на многообразии $X$. На самом деле утверждение теоремы А.3 может быть уточнено следующим способом.

Теорема А.4. Пусть $L$ - линейное расслоение на компактном комплексном многообразии $X$ с особой эрмитовой метрикой $h$ такой, что $\Theta_{L, h} \geqslant \varepsilon \omega$ для некоторого $\varepsilon>0$ и некоторой положительно определенной эрмитовой $(1,1)$-формы $\omega$ на $X$. Для каждого вещественного числа $m>0$ рассмотрим пространство $\mathscr{H}_{m}=H^{0}\left(X, L^{\otimes m} \otimes \mathscr{I}\left(h^{m}\right)\right)$ голоморфных сечений $\sigma$ расслоения $L^{\otimes m}$ на $X$ таких, что

$$
\int_{X}\|\sigma\|_{h^{m}}^{2} d V_{\omega}=\int_{X}|\sigma|^{2} e^{-2 m \varphi} d V_{\omega}<+\infty
$$

где $d V_{\omega}=(m !)^{-1} \omega^{m}$ - эрмитова форма обгема. Тогда для $m \gg 1$ пространство $\mathscr{H}_{m}$ является ненулевым конечномерным гилъбертовым пространством и можно рассмотреть замкнутый $(1,1)$-поток

$$
T_{m}=\frac{i}{2 \pi} \partial \bar{\partial}\left(\frac{1}{2 m} \log \sum_{k}\left|g_{m, k}\right|^{2}\right)=\frac{i}{2 \pi} \partial \bar{\partial}\left(\frac{1}{2 m} \log \sum_{k}\left\|g_{m, k}\right\|_{h}^{2}\right)+\Theta_{L, h},
$$

где $\left(g_{m, k}\right)_{1 \leqslant k \leqslant N(m)}$ - ортонормированный базис в $\mathscr{H}_{m}$. При этом выполнены следующие утверждения.

(i) Для любой тривиализачии $\left.L\right|_{U} \simeq U \times \mathbb{C}$ на координатном открытом множестве $U$ в $X$ и любого компактного множества $K \subset U$ найдутся константы $C_{1}, C_{2}>0$, не зависящие от $m u \varphi$, такие, что

$$
\varphi(z)-\frac{C_{1}}{m} \leqslant \psi_{m}(z):=\frac{1}{2 m} \log \sum_{k}\left|g_{m, k}(z)\right|^{2} \leqslant \sup _{|x-z|<r} \varphi(x)+\frac{1}{m} \log \frac{C_{2}}{r^{n}}
$$

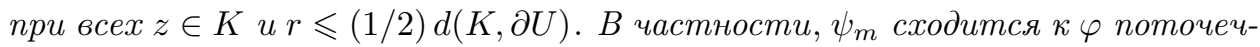
но и в $L_{\mathrm{loc}}^{1}-$ топологии на $\Omega$ при $m \rightarrow+\infty ;$ следовательно, $T_{m}$ слабо сходится $\kappa T=\Theta_{L, h}$.

(ii) Числа Лелона $\nu(T, z)=\nu(\varphi, z)$ и $\nu\left(T_{m}, z\right)=\nu\left(\psi_{m}, z\right)$ связаны неравенствами

$$
\nu(T, z)-n / m \leqslant \nu\left(T_{m}, z\right) \leqslant \nu(T, z) \quad \text { для всех } z \in X .
$$

(iii) Для любого компактного множества $K \subset X$ комплексные показатели особенностей метрик, локально заданных как $h=e^{-2 \varphi} u h_{m}=e^{-2 \psi_{m}}$, удовлетворяют неравенствам

$$
c_{K}(h)^{-1}-m^{-1} \leqslant c_{K}\left(h_{m}\right)^{-1} \leqslant c_{K}(h)^{-1} .
$$


ДокАЗАТЕЛьство. Большая часть доказательства получается небольшим изменением рассуждений из [63] (см. также [21; теорема 4.2]). Мы приводим здесь эти рассуждения для удобства читателя.

(i) Заметим, что $\sum\left|g_{m, k}(z)\right|^{2}$ является квадратом нормы линейной формы вычисления $\sigma \mapsto \sigma(z)$ на $\mathscr{H}_{m}$, так что

$$
\psi_{m}(z)=\sup _{\sigma \in B(1)} \frac{1}{m} \log |\sigma(z)|,
$$

где $B(1)$ - единичный шар в $\mathscr{H}_{m}$. При $r \leqslant(1 / 2) d(K, \partial \Omega)$ неравенство о среднем, примененное к плюрисубгармонической функции $|\sigma|^{2}$, дает

$$
\begin{aligned}
|\sigma(z)|^{2} & \leqslant \frac{1}{\pi^{n} r^{2 n} / n !} \int_{|x-z|<r}|\sigma(x)|^{2} d \lambda(x) \\
& \leqslant \frac{1}{\pi^{n} r^{2 n} / n !} \exp \left(2 m \sup _{|x-z|<r} \varphi(x)\right) \int_{\Omega}|\sigma|^{2} e^{-2 m \varphi} d \lambda .
\end{aligned}
$$

Если взять супремум по всем $\sigma \in B(1)$, то получится неравенство

$$
\psi_{m}(z) \leqslant \sup _{|x-z|<r} \varphi(x)+\frac{1}{2 m} \log \frac{1}{\pi^{n} r^{2 n} / n !},
$$

что дает второе неравенство из (i). Обратно, теорема Осавы-Такегоши о продолжении (см. [71], [72]), примененная к 0-мерному подмногообразию $\{z\} \subset U$, показывает, что для любого $a \in \mathbb{C}$ существует голоморфная функция $f$ на $U$ такая, что $f(z)=a$ и

$$
\int_{U}|f|^{2} e^{-2 m \varphi} d \lambda \leqslant C_{3}|a|^{2} e^{-2 m \varphi(z)},
$$

где $C_{3}$ зависит только от $n$ и $\operatorname{diam}(U)$. Далее, если $a$ остается в компактном множестве $K \subset U$, можно воспользоваться срезающей функцией $\theta$ с носителем в $U$, равной 1 в окрестности $a$, и решить $\bar{\partial}$-уравнение $\bar{\partial} g=\bar{\partial}(\theta f)$ в $L^{2}$-пространстве, ассоциированном с весом $2 m \varphi+2(n+1) \log |z-a|$, т. е. с особой эрмитовой метрикой $h(z)^{m}|z-a|^{-2(n+1)}$ на $L^{\otimes m}$. Для этого применим стандартные $L^{2}$-оценки Андреотти-Везентини-Хёрмандера (наиболее подходящую версию можно найти, например, в [73]). Благодаря предположению о том, что $\Theta_{L, h} \geqslant \varepsilon \omega>0$, это возможно при $m \geqslant m_{0}$, даже если многообразие $X$ не кэлерово (при этом из наших предположений следует, что $X$ является по крайней мере пространством Мойшезона). Число $m_{0}$ зависит от $\varepsilon$ и от геометрии конечного покрытия многообразия $X$ компактными подмножествами $K_{j} \subset U_{j}$, где $U_{j}-$ (например) координатные шары; оно не зависит от числа $a$ и даже от метрики $h$. Таким образом, $g(a)=0$ и, следовательно, $\sigma=\theta f-g$ является голоморфным сечением расслоения $L^{\otimes m}$ таким, что

$$
\int_{X}\|\sigma\|_{h^{m}}^{2} d V_{\omega}=\int_{X}|\sigma|^{2} e^{-2 m \varphi} d V_{\omega} \leqslant C_{4} \int_{U}|f|^{2} e^{-2 m \varphi} d V_{\omega} \leqslant C_{5}|a|^{2} e^{-2 m \varphi(z)} .
$$

В частности, $\sigma \in \mathscr{H}_{m}=H^{0}\left(X, L^{\otimes m} \otimes \mathscr{I}\left(h^{m}\right)\right)$. Зафиксируем такую точку $a$, что правая часть последнего неравенства равна 1 . Это дает неравенство

$$
\psi_{m}(z) \geqslant \frac{1}{m} \log |a|=\varphi(z)-\frac{\log C_{5}}{2 m},
$$

т. е. первое неравенство из (i). 
(ii) Первое неравенство из (i) влечет $\nu\left(\psi_{m}, z\right) \leqslant \nu(\varphi, z)$. С другой стороны, выполнено неравенство

$$
\sup _{|x-z|<r} \psi_{m}(x) \leqslant \sup _{|x-z|<2 r} \varphi(x)+\frac{1}{m} \log \frac{C_{2}}{r^{n}} .
$$

Разделим на $\log r<0$ и возьмем предел при $r$, стремящемся к 0 . Частное при делении на $\log r$ супремума плюрисубгармонической функции на $B(x, r)$ стремится к числу Лелона в $x$. Таким образом,

$$
\nu\left(\psi_{m}, x\right) \geqslant \nu(\varphi, x)-\frac{n}{m} .
$$

(iii) Первое неравенство из (i) сразу же дает оценку $h_{m} \leqslant C_{6} h$, так что $c_{K}\left(h_{m}\right) \geqslant c_{K}(h)$. Так как $c_{\cup K_{j}}(h)=\min _{j} c_{K_{j}}(h)$, для доказательства обратного неравенства можно без ограничения общности предполагать, что $K$ содержится в тривиализующем открытом множестве $U$ расслоения $L$. Выберем число $c<$ $c_{K}\left(\psi_{m}\right)$. Тогда, по определению, если $V \subset X$ - достаточно малая открытая окрестность $K$, то из неравенства Гёльдера для сопряженных экспонент $p=$ $1+m c^{-1}$ и $q=1+m^{-1} c$ следует, благодаря равенству $1 / p=c /(m q)$, что

$$
\begin{aligned}
\int_{V} e^{-2(m / p) \varphi} d V_{\omega}=\int_{V}\left(\sum_{1 \leqslant k \leqslant N(m)}\left|g_{m, k}\right|^{2} e^{-2 m \varphi}\right)^{1 / p} & \\
& \times\left(\sum_{1 \leqslant k \leqslant N(m)}\left|g_{m, k}\right|^{2}\right)^{-c /(m q)} d V_{\omega} \\
\leqslant & \left(\int_{X} \sum_{1 \leqslant k \leqslant N(m)}\left|g_{m, k}\right|^{2} e^{-2 m \varphi} d V_{\omega}\right)^{1 / p} \\
& \times\left(\int_{V}\left(\sum_{1 \leqslant k \leqslant N(m)}\left|g_{m, k}\right|^{2}\right)^{-c / m} d V_{\omega}\right)^{1 / q} \\
= & N(m)^{1 / p}\left(\int_{V}\left(\sum_{1 \leqslant k \leqslant N(m)}\left|g_{m, k}\right|^{2}\right)^{-c / m} d V_{\omega}\right)^{1 / q}<+\infty .
\end{aligned}
$$

Отсюда можно заключить, что $c_{K}(h) \geqslant m / p$, т. е. $c_{K}(h)^{-1} \leqslant p / m=1 / m+c^{-1}$. Так как число $c<c_{K}\left(\psi_{m}\right)$ было выбрано произвольно, то мы получаем $c_{K}(h)^{-1} \leqslant 1 / m+c_{K}\left(h_{m}\right)^{-1}$, и неравенства из (iii) доказаны.

ДокАЗАТЕЛЬСТво тЕОРЕмы А.3. Для данного объемного линейного расслоения $L$ на многообразии $X$ существует бирациональный морфизм $\mu: \widetilde{X} \rightarrow X$ такой, что многообразие $\widetilde{X}$ неособо и проективно и выполнена эквивалентность

$$
\mu^{*}(L) \sim A+E,
$$

где $A$ - обильный дивизор, а $E$ - эффективный $\mathbb{Q}$-дивизор. Рассматривая прямой образ относительно морфизма $\mu$ гладкой метрики $h_{A}$ на линейном расслоении $A$, имеющей положительную кривизну, мы получаем особую эрмитову метрику $h_{1}$ на линейном расслоении $L$ такую, что

$$
\Theta_{L, h_{1}} \geqslant \mu_{*} \Theta_{A, h_{A}} \geqslant \varepsilon \omega
$$


на многообразии $X$. В этом случае для любого $\delta>0$ и любой особой эрмитовой метрики $h$ на линейном расслоении $L$ такой, что $\Theta_{L, h} \geqslant 0$, интерполированная метрика $h_{\delta}=h_{1}^{\delta} h^{1-\delta}$ удовлетворяет условию $\Theta_{L, h_{\delta}} \geqslant \delta \varepsilon \omega$. Поскольку метрика $h_{1}$ ограничена вне 0 , мы видим, что $c_{K}(h) \geqslant(1-\delta) c_{K}\left(h_{\delta}\right)$ в силу монотонности. Применяя теорему A.4 (iii) к метрике $h_{\delta}$, мы видим, что

$$
c_{K}\left(h_{\delta}\right)=\lim _{m \rightarrow+\infty} c_{K}\left(h_{\delta, m}\right),
$$

а также

$$
c_{K}\left(h_{\delta, m}\right) \geqslant \operatorname{lct}_{K}\left(\frac{1}{m} D_{\delta, m}\right)
$$

для любого дивизора $D_{\delta, m}$, который задается нулями сечения $\sigma \in H^{0}\left(X, L^{\otimes m} \otimes\right.$ $\left.\mathscr{I}\left(h_{\delta}^{m}\right)\right)$, поскольку метрика $h_{\delta, m}$ задается посредством соотношения

$$
h_{\delta, m}=\left(\sum_{k}\left|g_{m, k}\right|^{2}\right)^{-1 / m}
$$

для ортонормированного базиса таких сечений. Отсюда сразу следует, что

$$
c_{K}(h) \geqslant \liminf _{\delta \rightarrow 0} \liminf _{m \rightarrow+\infty} \operatorname{lct}_{K}\left(\frac{1}{m} D_{\delta, m}\right) \geqslant \inf _{m \in \mathbb{Z}_{>0}} \inf _{D \in|m L|} \operatorname{lct}_{K}\left(\frac{1}{m} D\right) .
$$

Теорема А.3 доказана.

Во многих применениях мы сталкиваемся с точным действием конечной или компактной группы $G$ на многообразии $X$. В этом случае можно рассмотреть такие $G$-инвариантные объекты, как $G$-эквивариантные метрики на $G$-эквивариантном линейном расслоении $L$. В случае редуктивной алгебраической группы $G$ мы будем рассматривать вещественную максимальную компактную подгруппу $G^{\mathbb{R}}$ вместо самой группы $G$.

Отметим, что $G$-инвариантный $\alpha$-инвариант $\alpha_{G, K}(L)$ может быть естественным образом определен с помощью $G$-эквивариантных метрик в определении А.2. В этом случае все необходимые конструкции естественным образом обобщаются на $G$-эквивариантный случай. В частности, рассматриваемая линейная подсистема $\mathscr{H}_{m} \subset|m L|$ в данном случае будет $G$-инвариантной. Таким образом, для каждого $G$-инвариантного компактного подмножества $K \subset X$ мы получаем

$$
\begin{aligned}
\alpha_{G, K}(L) & =\inf _{\left\{\text {метрика } h G \text { эквивариантна, } \Theta_{L, h} \geqslant 0\right\}} c_{K}(h) \\
& =\inf _{m \in \mathbb{Z}>0} \inf _{|\Sigma| \subset|m L|, \Sigma^{G}=\Sigma} \operatorname{lct}_{K}\left(\frac{1}{m}|\Sigma|\right) .
\end{aligned}
$$

В случае, когда группа $G$ конечна, при $m \gg 0$ можно выбрать $G$-инвариантный дивизор $D_{\delta, m}$, который может быть задан обращением в нуль некоторого $G$-инвариантного сечения $\sigma$, возможно после домножения $m$ на порядок группы $G$. В этом случае можно получить более простое равенство

$$
\alpha_{G, K}(L)=\inf _{m \in \mathbb{Z}_{>0}} \inf _{D \in|m L|^{G}} \operatorname{lct}_{K}\left(\frac{1}{m} D\right) .
$$


Аналогично, вместо неособого многообразия можно рассмотреть многообразие $X$, имеющее не более чем факторособенности. В этом случае можно применять $L^{2}$-методы практически без каких-либо дополнительных изменений. Действительно, легко видеть, что все $L^{2}$-неравенства остаются верными при допущении факторособенностей, поскольку мы можем использовать орбифолдную метрику на открытой окрестности каждой точки.

\section{Приложение В. Большая таблица}

Настоящее приложение содержит список неособых трехмерных многообразий Фано. Мы придерживаемся обозначений и нумерации, принятых в [2], [50], [51]. Мы также используем следующие соглашения. Символ $V_{i}$ обозначает неособое трехмерное многообразие Фано такое, что $-K_{X} \sim 2 H$ и $\operatorname{Pic}\left(V_{i}\right)=$ $\mathbb{Z}[H]$, где $H$ - дивизор Картье на $V_{i}$ и $H^{3}=8 i \in\{8,16, \ldots, 40\}$. Символ $W$ обозначает (неособый) дивизор бистепени $(1,1)$ на $\mathbb{P}^{2} \times \mathbb{P}^{2}$ (или, что то же самое, многообразие $\left.\mathbb{P}\left(T_{\mathbb{P}^{2}}\right)\right)$. Символ $V_{7}$ обозначает раздутие $\mathbb{P}^{3}$ в точке (или, что то же самое, многообразие $\left.\mathbb{P}\left(\mathscr{O}_{\mathbb{P}^{2}} \oplus \mathscr{O}_{\mathbb{P}^{2}}(1)\right)\right)$. Символ $Q$ обозначает неособую трехмерную квадрику. Символ $S_{i}$ означает неособую поверхность дель Пеццо степени $K_{S_{i}}^{2}=i \in\{1, \ldots, 8\}$, причем $S_{8} ¥ \mathbb{P}^{1} \times \mathbb{P}^{1}$.

В четвертом столбце таблицы 1 содержатся значения глобальных лог-ка-

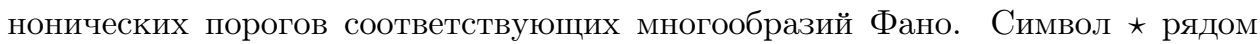
с числом означает, что порог $\operatorname{lct}(X)$ вычислен только для общего многообразия $X$ из данного деформационного класса. Если нам известна только верхняя оценка $\operatorname{lct}(X) \leqslant \alpha$, то мы пишем $\leqslant \alpha$ вместо точного значения порога $\operatorname{lct}(X)$; символ "?" означает, что мы не знаем никакой разумной оценки сверху (кроме тривиальной оценки $\operatorname{lct}(X) \leqslant 1)$.

Таблица 1: Неособые трехмерные многообразия Фано

\begin{tabular}{|c|c|c|c|}
\hline$I(X)$ & $-K_{X}^{3}$ & Краткое описание & $\operatorname{lct}(X)$ \\
\hline 1.1 & 2 & гиперповерхность степени 6 в $\mathbb{P}(1,1,1,1,3)$ & $1 \star$ \\
\hline 1.2 & 4 & $\begin{array}{l}\text { гиперповерхность степени } 4 \text { в } \mathbb{P}^{4} \text { или двойное накрытие } \\
\text { квадрики в } \mathbb{P}^{4}, \text { разветвленное в поверхности степени } 8\end{array}$ & $?$ \\
\hline 1.3 & 6 & полное пересечение квадрики и кубики в $\mathbb{P}^{5}$ & $?$ \\
\hline 1.4 & 8 & полное пересечение трех квадрик в $\mathbb{P}^{6}$ & $?$ \\
\hline 1.5 & 10 & $\begin{array}{l}\text { сечение грассманиана } \operatorname{Gr}(2,5) \subset \mathbb{P}^{9} \text { квадрикой и линей- } \\
\text { ным пространством размерности } 7\end{array}$ & $?$ \\
\hline 1.6 & 12 & $\begin{array}{l}\text { сечение эрмитова симметрического пространства } M= \\
G / P \subset \mathbb{P}^{15} \text { типа DIII линейным подпространством раз- } \\
\text { мерности } 8\end{array}$ & $?$ \\
\hline 1.7 & 14 & $\begin{array}{l}\text { сечение грассманиана } \operatorname{Gr}(2,6) \subset \mathbb{P}^{14} \text { линейным простран- } \\
\text { ством коразмерности } 5\end{array}$ & $?$ \\
\hline 1.8 & 16 & $\begin{array}{l}\text { сечение эрмитова симметрического пространства } M= \\
G / P \subset \mathbb{P}^{19} \text { типа CI линейным подпространством размер- } \\
\text { ности } 10\end{array}$ & $\leqslant 6 / 7$ \\
\hline
\end{tabular}




\begin{tabular}{|c|c|c|c|}
\hline 1.9 & 18 & $\begin{array}{l}\text { сечение } 5 \text {-мерного рационального однородного контакт- } \\
\text { ного многообразия } G_{2} / P \subset \mathbb{P}^{13} \text { линейным подпростран- } \\
\text { ством размерности } 11\end{array}$ & $\leqslant 4 / 5$ \\
\hline 1.10 & 22 & $\begin{array}{l}\text { множество совместных нулей трех сечений векторного } \\
\text { расслоения } \bigwedge^{2} \mathscr{Q} \text { ранга } 3, \text { где } \mathscr{Q}-\text { универсальное фак- } \\
\text { торрасслоение на грассманиане } \operatorname{Gr}(7,3)\end{array}$ & $\leqslant 2 / 3$ \\
\hline 1.11 & 8 & $V_{1}$, т. е. гиперповерхность степени 6 в $\mathbb{P}(1,1,1,2,3)$ & $1 / 2$ \\
\hline 1.12 & 16 & $V_{2}$, т. е. гиперповерхность степени 4 в $\mathbb{P}(1,1,1,1,2)$ & $1 / 2$ \\
\hline 1.13 & 24 & $V_{3}$, т. е. гиперповерхность степени 3 в $\mathbb{P}^{4}$ & $1 / 2$ \\
\hline 1.14 & 32 & $V_{4}$, т. е. полное пересечение двух квадрик в $\mathbb{P}^{5}$ & $1 / 2$ \\
\hline 1.15 & 40 & $\begin{array}{l}V_{5}, \text { т. е. сечение грассманиана } \operatorname{Gr}(2,5) \subset \mathbb{P}^{9} \text { линейным } \\
\text { пространством коразмерности } 3\end{array}$ & $1 / 2$ \\
\hline 1.16 & 54 & $Q$, т. е. гиперповерхность степени 2 в $\mathbb{P}^{4}$ & $1 / 3$ \\
\hline 1.17 & 64 & $\mathbb{P}^{3}$ & $1 / 4$ \\
\hline 2.1 & 4 & $\begin{array}{l}\text { раздутие многообразия Фано } V_{1} \text { в эллиптической кривой, } \\
\text { являющейся пересечением двух дивизоров из линейной } \\
\text { системы }\left|-\frac{1}{2} K_{V_{1}}\right|\end{array}$ & $1 / 2$ \\
\hline 2.2 & 6 & $\begin{array}{l}\text { двойное накрытие } \mathbb{P}^{1} \times \mathbb{P}^{2}, \text { разветвленное в дивизоре би- } \\
\text { степени }(2,4)\end{array}$ & $\leqslant 13 / 14$ \\
\hline 2.3 & 8 & $\begin{array}{l}\text { раздутие многообразия Фано } V_{2} \text { в эллиптической кривой, } \\
\text { являющейся пересечением двух дивизоров из линейной } \\
\text { системы }\left|-\frac{1}{2} K_{V_{2}}\right|\end{array}$ & $1 / 2$ \\
\hline 2.4 & 10 & раздутие $\mathbb{P}^{3}$ в пересечении двух кубик & $3 / 4 \star$ \\
\hline 2.5 & 12 & $\begin{array}{l}\text { раздутие многообразия } V_{3} \subset \mathbb{P}^{4} \text { в плоской кубической } \\
\text { кривой }\end{array}$ & $1 / 2 \star$ \\
\hline 2.6 & 12 & $\begin{array}{l}\text { дивизор бистепени }(2,2) \text { на } \mathbb{P}^{2} \times \mathbb{P}^{2} \text { или двойное накрытие } \\
\text { многообразия } W, \text { разветвленное в поверхности из линей- } \\
\text { ной системы }\left|-K_{W}\right|\end{array}$ & $?$ \\
\hline 2.7 & 14 & $\begin{array}{l}\text { раздутие } Q \text { в пересечении двух дивизоров из линейной } \\
\text { системы }\left|\mathscr{O}_{Q}(2)\right|\end{array}$ & $\leqslant 2 / 3$ \\
\hline 2.8 & 14 & $\begin{array}{l}\text { двойное накрытие } V_{7} \text {, разветвленное в дивизоре из ли- } \\
\text { нейной системы }\left|-K_{V_{7}}\right|\end{array}$ & $1 / 2 \star$ \\
\hline 2.9 & 16 & $\begin{array}{l}\text { раздутие } \mathbb{P}^{3} \text { в кривой степени } 7 \text { рода } 5 \text {, являющейся пе- } \\
\text { ресечением кубик }\end{array}$ & $\leqslant 3 / 4$ \\
\hline 2.10 & 16 & $\begin{array}{l}\text { раздутие } V_{4} \subset \mathbb{P}^{5} \text { в эллиптической кривой, являющейся } \\
\text { пересечением двух гиперплоских сечений }\end{array}$ & $1 / 2 \star$ \\
\hline 2.11 & 18 & раздутие $V_{3}$ в прямой & $1 / 2 \star$ \\
\hline 2.12 & 20 & $\begin{array}{l}\text { раздутие } \mathbb{P}^{3} \text { в кривой степени } 6 \text { рода } 3 \text {, являющейся пе- } \\
\text { ресечением кубик }\end{array}$ & $\leqslant 3 / 4$ \\
\hline 2.13 & 20 & раздутие $Q \subset \mathbb{P}^{4}$ в кривой степени 6 рода 2 & $\leqslant 2 / 3$ \\
\hline 2.14 & 20 & $\begin{array}{l}\text { раздутие } V_{5} \subset \mathbb{P}^{6} \text { в эллиптической кривой, являющейся } \\
\text { пересечением двух гиперплоских сечений }\end{array}$ & $1 / 2 \star$ \\
\hline 2.15 & 22 & раздутие $\mathbb{P}^{3}$ в пересечении поверхностей степени 2 и 3 & $1 / 2 \star$ \\
\hline 2.16 & 22 & раздутие $V_{4} \subset \mathbb{P}^{5}$ в конике & $\leqslant 1 / 2$ \\
\hline 2.17 & 24 & $\begin{array}{l}\text { раздутие } Q \subset \mathbb{P}^{4} \text { в нормальной эллиптической кривой } \\
\text { степени } 5\end{array}$ & $\leqslant 2 / 3$ \\
\hline
\end{tabular}




\begin{tabular}{|c|c|c|c|}
\hline 2.18 & 24 & $\begin{array}{l}\text { двойное накрытие } \mathbb{P}^{1} \times \mathbb{P}^{2}, \text { разветвленное в дивизоре би- } \\
\text { степени }(2,2)\end{array}$ & $1 / 2$ \\
\hline 2.19 & 26 & раздутие $V_{4} \subset \mathbb{P}^{5}$ в прямой & $1 / 2 \star$ \\
\hline 2.20 & 26 & раздутие $V_{5} \subset \mathbb{P}^{6}$ в скрученной кубике & $\leqslant 1 / 2$ \\
\hline 2.21 & 28 & раздутие $Q \subset \mathbb{P}^{4}$ в нормальной рациональной квартике & $\leqslant 2 / 3$ \\
\hline 2.22 & 30 & раздутие $V_{5} \subset \mathbb{P}^{6}$ в конике & $\leqslant 1 / 2$ \\
\hline 2.23 & 30 & $\begin{array}{l}\text { раздутие } Q \subset \mathbb{P}^{4} \text { в кривой степени } 4, \text { являющейся } \\
\text { пересечением поверхности из }\left|\mathscr{O}_{\mathbb{P}^{4}}(1)\right|_{Q} \mid \text { и поверхности } \\
\text { из }\left|\mathscr{O}_{\mathbb{P}^{4}}(2)\right|_{Q} \mid\end{array}$ & $1 / 3 \star$ \\
\hline 2.24 & 30 & дивизор бистепени $(1,2)$ на $\mathbb{P}^{2} \times \mathbb{P}^{2}$ & $1 / 2 \star$ \\
\hline 2.25 & 32 & $\begin{array}{l}\text { раздутие } \mathbb{P}^{3} \text { в эллиптической кривой, являющейся пере- } \\
\text { сечением двух квадрик }\end{array}$ & $1 / 2$ \\
\hline 2.26 & 34 & раздутие $V_{5} \subset \mathbb{P}^{6}$ в прямой & $1 / 2 \star$ \\
\hline 2.27 & 38 & раздутие $\mathbb{P}^{3}$ в скрученной кубике & $1 / 2$ \\
\hline 2.28 & 40 & раздутие $\mathbb{P}^{3}$ в плоской кубике & $1 / 4$ \\
\hline 2.29 & 40 & раздутие $Q \subset \mathbb{P}^{4}$ в конике & $1 / 3$ \\
\hline 2.30 & 46 & раздутие $\mathbb{P}^{3}$ в конике & $1 / 4$ \\
\hline 2.31 & 46 & раздутие $Q \subset \mathbb{P}^{4}$ в прямой & $1 / 3$ \\
\hline 2.32 & 48 & $W$, т. е. дивизор бистепени $(1,1)$ на $\mathbb{P}^{2} \times \mathbb{P}^{2}$ & $1 / 2$ \\
\hline 2.33 & 54 & раздутие $\mathbb{P}^{3}$ в прямой & $1 / 4$ \\
\hline 2.34 & 54 & $\mathbb{P}^{1} \times \mathbb{P}^{2}$ & $1 / 3$ \\
\hline 2.35 & 56 & $V_{7} \cong \mathbb{P}\left(\mathscr{O}_{\mathbb{P}^{2}} \oplus \mathscr{O}_{\mathbb{P}^{2}}(1)\right)$ & $1 / 4$ \\
\hline 2.36 & 62 & $\mathbb{P}\left(\mathscr{O}_{\mathbb{P}^{2}} \oplus \mathscr{O}_{\mathbb{P}^{2}}(2)\right)$ & $1 / 5$ \\
\hline 3.1 & 12 & $\begin{array}{l}\text { двойное накрытие } \mathbb{P}^{1} \times \mathbb{P}^{1} \times \mathbb{P}^{1}, \text { разветвленное в дивизоре } \\
\text { тристепени }(2,2,2)\end{array}$ & $3 / 4 \star$ \\
\hline 3.2 & 14 & $\begin{array}{l}\text { дивизор на } \mathbb{P}^{2} \text {-расслоении } \mathbb{P}\left(\mathscr{O}_{\mathbb{P}^{1} \times \mathbb{P}^{1}} \oplus \mathscr{O}_{\mathbb{P}^{1} \times \mathbb{P}^{1}}(-1,-1) \oplus\right. \\
\left.\mathscr{O}_{\mathbb{P}^{1} \times \mathbb{P}^{1}}(-1,-1)\right) \text { такой, что } X \in\left|L^{\otimes 2} \otimes \mathscr{O}_{\mathbb{P}^{1} \times \mathbb{P}^{1}}(2,3)\right| \text {, } \\
\text { где } L \text { - тавтологическое линейное расслоение }\end{array}$ & $1 / 2 \star$ \\
\hline 3.3 & 18 & дивизор тристепени $(1,1,2)$ на $\mathbb{P}^{1} \times \mathbb{P}^{1} \times \mathbb{P}^{2}$ & $2 / 3 \star$ \\
\hline 3.4 & 18 & $\begin{array}{l}\text { раздутие трехмерного многообразия Фано } Y \text { с } \Xi(Y)=2.18 \\
\text { в неособом слое композиции } Y \rightarrow \mathbb{P}^{1} \times \mathbb{P}^{2} \rightarrow \mathbb{P}^{2} \text { двойного } \\
\text { накрытия и проекции }\end{array}$ & $1 / 2$ \\
\hline 3.5 & 20 & $\begin{array}{l}\text { раздутие } \mathbb{P}^{1} \times \mathbb{P}^{2} \text { в кривой } C \text { бистепени }(5,2) \text { такой, что } \\
\text { композиция } C \hookrightarrow \mathbb{P}^{1} \times \mathbb{P}^{2} \rightarrow \mathbb{P}^{2} \text { является вложением }\end{array}$ & $1 / 2 \star$ \\
\hline 3.6 & 22 & $\begin{array}{l}\text { раздутие } \mathbb{P}^{3} \text { в несвязном объединении прямой и нормаль- } \\
\text { ной эллиптической кривой степени } 4\end{array}$ & $1 / 2 \star$ \\
\hline 3.7 & 24 & $\begin{array}{l}\text { раздутие многообразия } W \text { в эллиптической кривой, яв- } \\
\text { ляющейся пересечением двух дивизоров из линейной си- } \\
\text { стемы }\left|-\frac{1}{2} K_{W}\right|\end{array}$ & $1 / 2 \star$ \\
\hline 3.8 & 24 & $\begin{array}{l}\text { дивизор из линейной системы } \mid\left(\alpha \circ \pi_{1}\right)^{*}\left(\mathscr{O}_{\mathbb{P}^{2}}(1)\right) \otimes \\
\pi_{2}^{*}\left(\mathscr{O}_{\mathbb{P}^{2}}(2)\right) \mid, \text { где } \pi_{1}: \mathscr{F}_{1} \times \mathbb{P}^{2} \rightarrow \mathscr{F}_{1} \text { и } \pi_{2}: \mathscr{F}_{1} \times \mathbb{P}^{2} \rightarrow \mathbb{P}^{2}- \\
\text { проекции, а } \alpha: \mathscr{F}_{1} \rightarrow \mathbb{P}^{2}-\text { раздутие точки }\end{array}$ & $1 / 2 \star$ \\
\hline 3.9 & 26 & $\begin{array}{l}\text { раздутие конуса } W_{4} \subset \mathbb{P}^{6} \text { над поверхностью Веронезе } \\
R_{4} \subset \mathbb{P}^{5} \text { с центром в несвязном объединении вершины } \\
\text { и квартики на } R_{4} \cong \mathbb{P}^{2}\end{array}$ & $1 / 3$ \\
\hline 3.10 & 26 & раздутие $Q \subset \mathbb{P}^{4}$ в несвязном объединении двух коник & $1 / 2$ \\
\hline
\end{tabular}




\begin{tabular}{|c|c|c|c|}
\hline 3.11 & 28 & $\begin{array}{l}\text { раздутие многообразия } V_{7} \text { в эллиптической кривой, яв- } \\
\text { ляющейся пересечением двух дивизоров из линейной си- } \\
\text { стемы }\left|-\frac{1}{2} K_{V_{7}}\right|\end{array}$ & $1 / 2$ \\
\hline 3.12 & 28 & $\begin{array}{l}\text { раздутие } \mathbb{P}^{3} \text { в несвязном объединении прямой и скручен- } \\
\text { ной кубики }\end{array}$ & $1 / 2$ \\
\hline 3.13 & 30 & $\begin{array}{l}\text { раздутие } W \subset \mathbb{P}^{2} \times \mathbb{P}^{2} \text { в кривой } C \text { бистепени }(2,2) \text { такой, } \\
\text { что } \pi_{1}(C) \subset \mathbb{P}^{2} \text { и } \pi_{2}(C) \subset \mathbb{P}^{2} \text { являются неприводимыми } \\
\text { кониками, где } \pi_{1}: W \rightarrow \mathbb{P}^{2} \text { и } \pi_{2}: W \rightarrow \mathbb{P}^{2}-\text { естественные } \\
\text { проекции }\end{array}$ & $\leqslant 1 / 2$ \\
\hline 3.14 & 32 & $\begin{array}{l}\text { раздутие } \mathbb{P}^{3} \text { в несвязном объединении плоской кубиче- } \\
\text { ской кривой, содержащейся в плоскости П } \subset \mathbb{P}^{3}, \text { и точки, } \\
\text { не содержащейся в П }\end{array}$ & $1 / 2$ \\
\hline 3.15 & 32 & $\begin{array}{l}\text { раздутие } Q \subset \mathbb{P}^{4} \text { в несвязном объединении прямой и ко- } \\
\text { ники }\end{array}$ & $1 / 2$ \\
\hline 3.16 & 34 & $\begin{array}{l}\text { раздутие } V_{7} \text { в собственном прообразе при раздутии } \\
\alpha: V_{7} \rightarrow \mathbb{P}^{3} \text { скрученной кубики, проходящей через центр } \\
\text { раздутия } \alpha\end{array}$ & $1 / 2$ \\
\hline 3.17 & 36 & дивизор тристепени $(1,1,1)$ на $\mathbb{P}^{1} \times \mathbb{P}^{1} \times \mathbb{P}^{2}$ & $1 / 2$ \\
\hline 3.18 & 36 & раздутие $\mathbb{P}^{3}$ в несвязном объединении прямой и коники & $1 / 3$ \\
\hline 3.19 & 38 & раздутие $Q \subset \mathbb{P}^{4}$ в двух неколлинеарных точках & $1 / 3$ \\
\hline 3.20 & 38 & раздутие $Q \subset \mathbb{P}^{4}$ в несвязном объединении двух прямых & $1 / 3$ \\
\hline 3.21 & 38 & раздутие $\mathbb{P}^{1} \times \mathbb{P}^{2}$ в кривой бистепени $(2,1)$ & $1 / 3$ \\
\hline 3.22 & 40 & $\begin{array}{l}\text { раздутие } \mathbb{P}^{1} \times \mathbb{P}^{2} \text { в конике, содержащейся в слое проекции } \\
\mathbb{P}^{1} \times \mathbb{P}^{2} \rightarrow \mathbb{P}^{1}\end{array}$ & $1 / 3$ \\
\hline 3.23 & 42 & $\begin{array}{l}\text { раздутие } V_{7} \text { в собственном прообразе при раздутии } \\
\alpha: V_{7} \rightarrow \mathbb{P}^{3} \text { неприводимой коники, проходящей через } \\
\text { центр раздутия } \alpha\end{array}$ & $1 / 4$ \\
\hline 3.24 & 42 & $\begin{array}{l}W \times_{\mathbb{P}^{2}} \mathbb{F}_{1} \text {, где отображение } W \rightarrow \mathbb{P}^{2} \text { является } \mathbb{P}^{1} \text {-расслое- } \\
\text { нием, а отображение } \mathbb{F}_{1} \rightarrow \mathbb{P}^{2}-\text { раздутием точки }\end{array}$ & $1 / 3$ \\
\hline 3.25 & 44 & раздутие $\mathbb{P}^{3}$ в несвязном объединении двух прямых & $1 / 3$ \\
\hline 3.26 & 46 & раздутие $\mathbb{P}^{3}$ в несвязном объединении точки и прямой & $1 / 4$ \\
\hline 3.27 & 48 & $\mathbb{P}^{1} \times \mathbb{P}^{1} \times \mathbb{P}^{1}$ & $1 / 2$ \\
\hline 3.28 & 48 & $\mathbb{P}^{1} \times \mathbb{F}_{1}$ & $1 / 3$ \\
\hline 3.29 & 50 & $\begin{array}{l}\text { раздутие } V_{7} \text { в прямой, лежащей на } E \cong \mathbb{P}^{2}, \text { где } E-\text { ис- } \\
\text { ключительный дивизор раздутия } V_{7} \rightarrow \mathbb{P}^{3}\end{array}$ & $1 / 5$ \\
\hline 3.30 & 50 & $\begin{array}{l}\text { раздутие } V_{7} \text { в собственном прообразе при раздутии } \\
\alpha: V_{7} \rightarrow \mathbb{P}^{3} \text { прямой, проходящей через центр раздутия } \alpha\end{array}$ & $1 / 4$ \\
\hline 3.31 & 52 & раздутие конуса над неособой квадрикой в $\mathbb{P}^{3}$ в вершине & $1 / 3$ \\
\hline 4.1 & 24 & дивизор мультистепени $(1,1,1,1)$ на $\mathbb{P}^{1} \times \mathbb{P}^{1} \times \mathbb{P}^{1} \times \mathbb{P}^{1}$ & $1 / 2$ \\
\hline 4.2 & 28 & $\begin{array}{l}\text { раздутие конуса над неособой квадрикой } S \subset \mathbb{P}^{3} \text { в несвяз- } \\
\text { ном объединении вершины и эллиптической кривой на } S\end{array}$ & $1 / 2$ \\
\hline 4.3 & 30 & раздутие $\mathbb{P}^{1} \times \mathbb{P}^{1} \times \mathbb{P}^{1}$ в кривой тристепени $(1,1,2)$ & $1 / 2$ \\
\hline 4.4 & 32 & $\begin{array}{l}\text { раздутие неособого трехмерного многообразия Фано } Y \\
\text { с } Ј(Y)=3.19 \text { в собственном прообразе коники, лежащей } \\
\text { на квадрике } Q \subset \mathbb{P}^{4} \text { и проходящей через оба центра раз- } \\
\text { дутия } Y \rightarrow Q\end{array}$ & $1 / 3$ \\
\hline
\end{tabular}




\begin{tabular}{|c|c|c|c|}
\hline 4.5 & 32 & $\begin{array}{l}\text { раздутие } \mathbb{P}^{1} \times \mathbb{P}^{2} \text { в несвязном объединении двух неприво- } \\
\text { димых кривых бистепеней }(2,1) \text { и }(1,0)\end{array}$ & $3 / 7$ \\
\hline 4.6 & 34 & раздутие $\mathbb{P}^{3}$ в несвязном объединении трех прямых & $1 / 2$ \\
\hline 4.7 & 36 & $\begin{array}{l}\text { раздутие } W \subset \mathbb{P}^{2} \times \mathbb{P}^{2} \text { в несвязном объединении двух } \\
\text { кривых бистепеней }(0,1) \text { и }(1,0)\end{array}$ & $1 / 2$ \\
\hline 4.8 & 38 & раздутие $\mathbb{P}^{1} \times \mathbb{P}^{1} \times \mathbb{P}^{1}$ в кривой тристепени $(0,1,1)$ & $1 / 3$ \\
\hline 4.9 & 40 & $\begin{array}{l}\text { раздутие неособого трехмерного многообразия Фано } Y \text { с } \\
I(Y)=3.25 \text { в кривой, стягиваемой отображением } Y \rightarrow \mathbb{P}^{3}\end{array}$ & $1 / 3$ \\
\hline 4.10 & 42 & $\mathbb{P}^{1} \times S_{7}$ & $1 / 3$ \\
\hline 4.11 & 44 & $\begin{array}{l}\text { раздутие } \mathbb{P}^{1} \times \mathbb{F}_{1} \text { в кривой } C \cong \mathbb{P}^{1} \text { такой, что } C \text { содержится } \\
\text { в слое } F \cong \mathbb{F}_{1} \text { проекции } \mathbb{P}^{1} \times \mathbb{F}_{1} \rightarrow \mathbb{P}^{1} \text { и } C \cdot C=-1 \text { на } F\end{array}$ & $1 / 3$ \\
\hline 4.12 & 46 & $\begin{array}{l}\text { раздутие неособого трехмерного многообразия Фано } Y \\
\text { с } I(Y)=2.33 \text { в двух кривых, стягиваемых отображением } \\
Y \rightarrow \mathbb{P}^{3}\end{array}$ & $1 / 4$ \\
\hline 4.13 & 26 & раздутие $\mathbb{P}^{1} \times \mathbb{P}^{1} \times \mathbb{P}^{1}$ в кривой тристепени $(1,1,3)$ & $1 / 2 \star$ \\
\hline 5.1 & 28 & $\begin{array}{l}\text { раздутие неособого трехмерного многообразия Фано } Y \\
\text { с } I(Y)=2.29 \text { в трех кривых, стягиваемых отображением } \\
Y \rightarrow Q\end{array}$ & $1 / 3$ \\
\hline 5.2 & 36 & $\begin{array}{l}\text { раздутие неособого трехмерного многообразия Фано } Y \\
\text { с } J(Y)=3.25 \text { в двух кривых } C_{1} \neq C_{2} \text {, стягиваемых отоб- } \\
\text { ражением } \varphi: Y \rightarrow \mathbb{P}^{3} \text { и содержащихся в одном и том же } \\
\text { исключительном дивизоре раздутия } \varphi\end{array}$ & $1 / 3$ \\
\hline 5.3 & 36 & $\mathbb{P}^{1} \times S_{6}$ & $1 / 2$ \\
\hline 5.4 & 30 & $\mathbb{P}^{1} \times S_{5}$ & $1 / 2$ \\
\hline 5.5 & 24 & $\mathbb{P}^{1} \times S_{4}$ & $1 / 2$ \\
\hline 5.6 & 18 & $\mathbb{P}^{1} \times S_{3}$ & $1 / 2$ \\
\hline 5.7 & 12 & $\mathbb{P}^{1} \times S_{2}$ & $1 / 2$ \\
\hline 5.8 & 6 & $\mathbb{P}^{1} \times S_{1}$ & $1 / 2$ \\
\hline
\end{tabular}

\section{Список литературы}

[1] J. Kollár, "Singularities of pairs", Algebraic geometry (Santa Cruz, 1995), Proc. Sympos. Pure Math., 62, Amer. Math. Soc., Providence, RI, 1997, 221-287.

[2] V. Iskovskikh, Yu. Prokhorov, "Fano varieties", Algebraic geometry, Vol. V, Encyclopaedia Math. Sci., 47, Springer, Berlin, 1999, 1-245.

[3] G. Tian, "On Kähler-Einstein metrics on certain Kähler manifolds with $c_{1}(M)>0$ ", Invent. Math., 89 (1987), 225-246.

[4] Q. Zhang, "Rational connectedness of $\log \mathbb{Q}$-Fano varieties", J. Reine Angew. Math., 590 (2006), 131-142.

[5] И. А. Чельцов, "Лог-канонические пороги на гиперповерхностях", Матем. сб., 192:8 (2001), 155-172; англ. пер.: I. A. Cheltsov, "Log canonical thresholds on hypersurfaces", Sb. Math., 192:8 (2001), 1241-1257.

[6] Jun-Muk Hwang, "Log canonical thresholds of divisors on Fano manifolds of Picard rank 1", Compos. Math., 143:1 (2007), 89-94.

[7] А. В. Пухликов, "Бирациональная геометрия прямых произведений Фано", Изв. РАН. Сер. матем., 69:6 (2005), 153-186; англ. пер.: A. V. Pukhlikov, "Birational geometry of Fano direct products", Izv. Math., 69:6 (2005), 1225-1255. 
[8] I. Cheltsov, J. Park, J. Won, Log canonical thresholds of certain Fano hypersurfaces, arXiv: abs/0706.0751v1, 2007.

[9] A. R. Iano-Fletcher, "Working with weighted complete intersections", Explicit birational geometry of 3-folds, London Math. Soc. Lecture Note Ser., 281, Cambridge Univ. Press, Cambridge, 2000, 101-173.

[10] J. M. Johnson, J. Kollár, "Fano hypersurfaces in weighted projective 4-spaces", Experiment. Math., 10:1 (2001), 151-158.

[11] I. Cheltsov, "Fano varieties with many selfmaps", Adv. Math., 217:1 (2008), 97-124.

[12] И. А. Чельцов, “Экстремальные метрики на двух многообразиях Фано”, Матем. сб. (в печати).

[13] И.А. Чельцов, “Двойные пространства с особенностями”, Матем. сб., 199:2 (2008), 131-148; англ. пер.: I. A. Cheltsov, "Double spaces with isolated singularities", Sb. Math., 199:2 (2008), 291-306.

[14] I. Cheltsov, "Log canonical thresholds of del Pezzo surfaces", Geom. Funct. Anal. (to appear).

[15] M. Furushima, "Singular del Pezzo surfaces and analytic compactifications of 3-dimensional complex affine space $C^{3 "}$, Nagoya Math. J., 104 (1986), 1-28.

[16] J. W. Bruce, C. T. C. Wall, "On the classification of cubic surfaces", J. London Math. Soc. (2), 19:2 (1979), 245-256.

[17] I. Cheltsov, On singular cubic surfaces, arXiv: abs/0706.2666, 2007.

[18] G. Tian, "On a set of polarized Kähler metrics on algebraic manifolds", J. Differential Geom., 32:1 (1990), 99-130.

[19] I. Dolgachev, V. Iskovskikh, Finite subgroups of the plane Cremona group, arXiv: abs/math/0610595v3, 2006.

[20] A.M. Nadel, "Multiplier ideal sheaves and Kähler-Einstein metrics of positive scalar curvature", Ann. of Math. (2), 132:3 (1990), 549-596.

[21] J.-P. Demailly, J. Kollár, "Semi-continuity of complex singularity exponents and Kähler-Einstein metrics on Fano orbifolds", Ann. Sci. École Norm. Sup. (4), 34:4 (2001), 525-556.

[22] T. Aubin, "Équations du type Monge-Ampère sur les variétés kählériennes compactes", Bull. Sci. Math. (2), 102:1 (1978), 63-95.

[23] S. T. Yau, "On the Ricci curvature of a compact Kähler manifold and the complex Monge-Ampère equation. I", Comm. Pure Appl. Math., 31:3 (1978), 339-411.

[24] S. T. Yau, "Review on Kähler-Einstein metrics in algebraic geometry", Proceedings of the Hirzebruch 65 Conference on Algebraic Geometry (Ramat Gan, 1993), Israel Math. Conf. Proc., 9, Bar-Ilan Univ., Ramat Gan, 1996, 433-443.

[25] Y. Matsushima, "Sur la structure du groupe d'homéomorphismes analytiques d'une certaine variété kählérienne", Nagoya Math. J., 11 (1957), 145-150.

[26] M. Lübke, "Stability of Einstein-Hermitian vector bundles", Manuscripta Math., 42:2-3 (1983), 245-257.

[27] A. Futaki, "An obstruction to the existence of Einstein-Kähler metrics", Invent. Math., 73:3 (1983), 437-443.

[28] A. Steffens, "On the stability of the tangent bundle of Fano manifolds", Math. Ann., 304:4 (1996), 635-643.

[29] T. Mabuchi, "Einstein-Kähler forms, Futaki invariants and convex geometry on toric Fano varieties", Osaka J. Math., 24 (1987), 705-737. 
[30] V. Batyrev, E. Selivanova, "Einstein-Kähler metrics on symmetric toric Fano manifolds", J. Reine Angew. Math., 512 (1999), 225-236.

[31] Xu-Jia Wang, Xiaohua Zhu, "Kähler-Ricci solitons on toric manifolds with positive first Chern class", Adv. Math., 188:1 (2004), 87-103.

[32] B. Nill, "Complete toric varieties with reductive automorphism group", Math. Z., 252:4 (2006), 767-786.

[33] G. Tian, "On Calabi's conjecture for complex surfaces with positive first Chern class", Invent. Math., 101:1 (1990), 101-172.

[34] H. Matsumura, P. Monsky, "On the automorphisms of hypersurfaces", J. Math. Kyoto Univ., 3 (1964), 347-361.

[35] C. Arezzo, A. Ghigi, G. Pirola, "Symmetries, quotients and Kähler-Einstein metrics", J. Reine Angew. Math., 591 (2006), 177-200.

[36] J. Park, "Birational maps of del Pezzo fibrations", J. Reine Angew. Math., 538 (2001), 213-221.

[37] A. Corti, "Del Pezzo surfaces over Dedekind schemes", Ann. of Math. (2), 144:3 (1996), 641-683.

[38] И. А. Чельцов, "Бирационально жесткие многообразия Фано”, УМH, 60:5 (2005), 71-160; англ. пер.: I. A. Cheltsov, "Birationally rigid Fano varieties", Russian Math. Surveys, 60:5 (2005), 875-965.

[39] C. Birkar, P. Cascini, C. Hacon, J. McKernan, Existence of minimal models for varieties of log general type, arXiv: math/0610203, 2006.

[40] В.В. Шокуров, "Трехмерные логперестройки", Изв. РАН. Сер. матем., 56:1 (1992), 105-203; англ. пер.: V. V. Shokurov, "3-fold log flips", Russian Acad. Sci. Izv. Math., 40:1 (1993), 875-965.

[41] J. Kollár, S. Mori, Birational geometry of algebraic varieties, Cambridge Tracts in Math., 134, Cambridge Univ. Press, Cambridge, 1998.

[42] Ю. И. Манин, "Рациональные поверхности над совершенными полями. II", Maтем. сб., 72(114):2 (1967), 161-192; англ. пер.: Yu. I. Manin, "Rational surfaces over perfect fields. II", Math. USSR Sb., 1:2 (1967), 141-169.

[43] A. V. Pukhlikov, "Birational automorphisms of Fano hypersurfaces", Invent. Math., 134:2 (1998), 401-426.

[44] В. А. Исковских, "Бирациональные автоморфизмы трехмерных алгебраических многообразий”, Итоги науки и техники. Соврем. пробл. матем., 12, ВИНИТИ, M., 1979, 159-236; англ. пер.: V.A. Iskovskikh, "Birational automorphisms of three-dimensional algebraic varieties", J. Math. Sci. (N.Y.), 13:6 (1980), 815-868.

[45] J. Kollár, "Birational rigidity of Fano varieties and field extensions", Тр. МИАН, 264, 2009 (в печати).

[46] A. Corti, A. Pukhlikov, M. Reid, "Fano 3-fold hypersurfaces", London Math. Soc. Lecture Note Ser., 281, Cambridge Univ. Press, Cambridge, 2000, 175-258.

[47] I. Cheltsov, J. Park, "Weighted Fano threefold hypersurfaces", J. Reine Angew. Math., 600 (2006), 81-116.

[48] В. А. Исковских, "Трехмерные многообразия Фано. I", Изв. АН СССР. Сер. матем., 41:3 (1977), 516-562; англ. пер.: V. A. Iskovskikh, "Fano 3-folds. I", Math. USSR-Izv., 11:3 (1977), 485-527.

[49] В. А. Исковских, “Трехмерные многообразия Фано. II”, Изв. АН СССР. Сер. матем., 42:3 (1978), 504-549; англ. пер.: V. A. Iskovskikh, "Fano 3-folds. II", Math. USSR-Izv., 12:3 (1978), 469-506.

[50] S. Mori, S. Mukai, "Classification of Fano 3-folds with $B_{2} \geqslant 2$ ", Manuscripta Math., 36:2 (1981), 147-162. 
[51] S. Mori, S. Mukai, "Erratum: 'Classification of Fano 3-folds with $B_{2} \geqslant 2$ '", Manuscripta Math., 110:3 (2003), 407.

[52] S. Mori, S. Mukai, "On Fano 3-folds with $B_{2} \geqslant 2$ ", Algebraic varieties and analytic varieties (Tokyo, 1981), Adv. Stud. Pure Math., 1, North-Holland, Amsterdam, 1983, 101-129.

[53] R. Lazarsfeld, Positivity in algebraic geometry. II. Positivity for vector bundles, and multiplier ideals, Ergeb. Math. Grenzgeb. (3), 49, Springer-Verlag, Berlin, 2004.

[54] E. Viehweg, Quasi-projective moduli for polarized manifolds, Ergeb. Math. Grenzgeb. (3), 30, Springer-Verlag, Berlin, 1995.

[55] J. Song, "The $\alpha$-invariant on toric Fano threefolds", Amer. J. Math., 127:6 (2005), 1247-1259.

[56] W. Fulton, "Introduction to toric varieties", The 1989 William H. Roever lectures in geometry, Ann. of Math. Stud., 131, Princeton Univ. Press, Princeton, NJ, 1993.

[57] В.Г. Саркисов, "Бирациональные автоморфизмы расслоений коник”, Изв. АН СССР. Сер. матем., 44:4 (1980), 918-945; англ. пер.: V. G. Sarkisov, "Birational automorphisms of conic bundles", Math. USSR-Izv., 17:1 (1981), 177-202.

[58] M. Szurek, J. Wiśniewski, "Fano bundles of rank 2 on surfaces", Algebraic geometry (Berlin, 1988), Compos. Math., 76, № 1-2, 1990, 295-305.

[59] P. Jahnke, T. Peternell, I. Radloff, Threefolds with big and nef anticanonical bundles. II, arXiv: 0710.2763, 2007.

[60] P. Jahnke, T. Peternell, Almost del Pezzo manifolds, arXiv: math/0612516, 2006.

[61] M. Furushima, "Singular Fano compactifications of $\mathbb{C}^{3}$. I", Math. Z., 248:4 (2004), 709-723.

[62] В.В. Шокуров, “Существование прямой на многообразии Фано”, Изв. АН СССР. Сер. матем., 43:4 (1979), 922-964; англ. пер.: V. V. Šokurov, "Birational automorphisms of conic bundles", Math. USSR-Izv., 15:1 (1980), 173-209.

[63] J.-P. Demailly, "Regularization of closed positive currents and intersection theory", J. Algebraic Geom., 1:3 (1992), 361-409.

[64] J. Johnson, J. Kollár, "Kähler-Einstein metrics on log del Pezzo surfaces in weighted projective 3-spaces", Ann. Inst. Fourier (Grenoble), 51:1 (2001), 69-79.

[65] C. Boyer, K. Galicki, J. Kollár, "Einstein metrics on spheres", Ann. of Math. (2), 162:1 (2005), 557-580.

[66] J.-P. Demailly, "Singular Hermitian metrics on positive line bundles", Complex algebraic varieties (Bayreuth, 1990), Lecture Notes in Math., 1507, Springer, Berlin, 1992, 87-104.

[67] А. Н. Варченко, "Комплексный показатель особенности не меняется вдоль страта $\mu=$ const", Функи. анализ и его прил., 16:1 (1982), 1-12; англ. пер.: А. N. Varchenko, "The complex exponent of a singularity does not change along strata $\mu=$ const", Funct. Anal. Appl., 16:1 (1982), 1-9.

[68] А.Н. Варченко, "О полунепрерывности комплексного показателя особенности", Функи. анализ и его прил., 17:4 (1983), 77-78; англ. пер.: А. N. Varchenko, "Semicontinuity of the complex singularity index", Funct. Anal. Appl., 17:4 (1983), 307-309.

[69] В. И. Арнольд, С. М. Гусейн-Заде, А.Н. Варченко, Особенности дифферениируемых отображений. II. Монодромия и асимптотики интегралов, Наука, М., 1984; англ. пер.: V. I. Arnol'd, S. M. Gusejn-Zade, A. N. Varchenko, Singularities of differentiable maps. Vol. II. Monodromy and asymptotics of integrals, Monogr. Math., 83, Birkhäuser, Boston, 1988. 
[70] Y. T. Siu, "The existence of Kähler-Einstein metrics on manifolds with positive anticanonical line bundle and a suitable finite symmetry group", Ann. of Math. (2), 127:3 (1988), 585-627.

[71] T. Ohsawa, K. Takegoshi, "On the extension of $L^{2}$ holomorphic functions", Math. Z., 195:2 (1987), 197-204.

[72] T. Ohsawa, "On the extension of $L^{2}$ holomorphic functions. II", Publ. Res. Inst. Math. Sci., 24:2 (1988), 265-275.

[73] J.-P. Demailly, "Estimations $L^{2}$ pour l'opérateur $\bar{\partial}$ d'un fibré vectoriel holomorphe semi-positif au-dessus d'une variété kählérienne complète", Ann. Sci. École Norm. Sup. (4), 15:3 (1982), 457-511.

И. А. Чельцов (I. A. Cheltsov)

Математический институт им. В. А. Стеклова РАН

E-mail: cheltsov@yahoo.com

\section{K. А. Шрамов (K. A. Shramov)}

Математический институт им. В. А. Стеклова РАН

E-mail: shramov@mccme.ru
Поступила в редакцию

26.07.2008 